\title{
Application of the Intramolecular Yamamoto Vinylogous Aldol Reaction to the Synthesis of Macrolides
}

\author{
Joseph A. Abramite and Tarek Sammakia* \\ Department of Chemistry and Biochemistry, University of Colorado, Boulder, Colorado \\ 80309-0215
}

Supporting Information

Table of Contents:

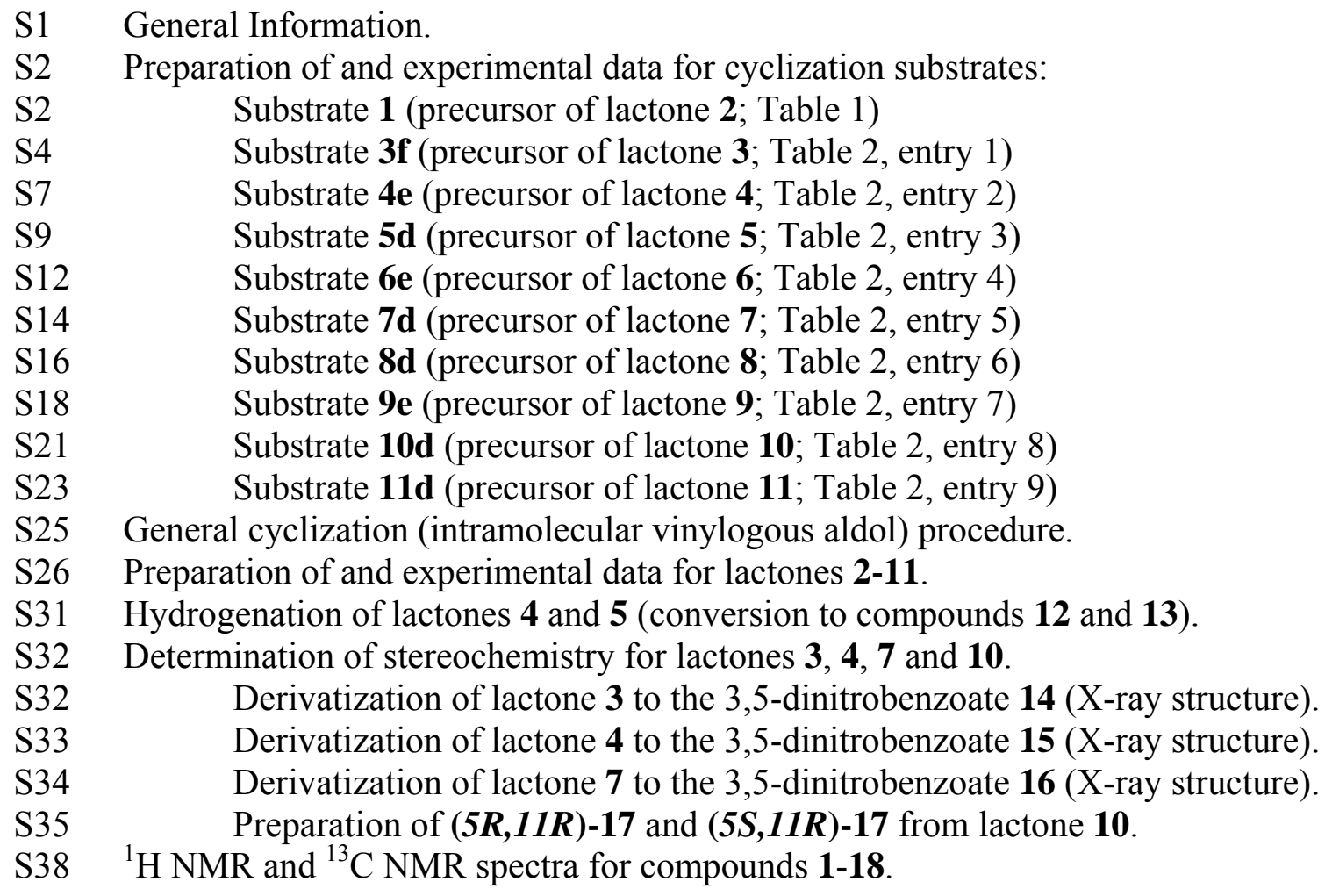

\section{General Information.}

All reactions were performed in oven-dried or flame-dried glassware under a dry nitrogen atmosphere. Toluene was washed with concentrated $\mathrm{H}_{2} \mathrm{SO}_{4}, \mathrm{H}_{2} \mathrm{O}, 1 \mathrm{M} \mathrm{NaOH}$, $\mathrm{H}_{2} \mathrm{O}$, dried over anhydrous $\mathrm{MgSO}_{4}$, filtered and distilled from $\mathrm{CaH}_{2}$ under nitrogen prior to use. $\mathrm{CH}_{2} \mathrm{Cl}_{2}, \mathrm{Et}_{3} \mathrm{~N}$, and 2,2,6,6-tetramethylpiperidine were distilled from $\mathrm{CaH}_{2}$ under nitrogen prior to use. DMSO was distilled from $\mathrm{CaH}_{2}$ and stored over $4 \AA$ molecular sieves prior to use. THF and $\mathrm{Et}_{2} \mathrm{O}$ were distilled from $\mathrm{Na}$ benzophenone ketyl under nitrogen prior to use. 2,6-Diphenylphenol was recrystallized twice from hexanes prior to use. All other chemicals were used as received from the supplier. Flash chromatography was performed using $60 \AA$ silica gel $(37-75 \mu \mathrm{m})$. ${ }^{1} \mathrm{H}$ NMR spectra were recorded at either $400 \mathrm{MHz}$ or $500 \mathrm{MHz}$ in $\mathrm{CDCl}_{3}$ using residual $\mathrm{CHCl}_{3}(7.24 \mathrm{ppm})$ as the internal 
reference. ${ }^{13} \mathrm{C}$ NMR spectra were recorded at $100 \mathrm{MHz}$ in $\mathrm{CDCl}_{3}$ using residual $\mathrm{CHCl}_{3}$ $(77.26 \mathrm{ppm})$ as the internal reference. Infrared (IR) spectra were obtained as thin flims on $\mathrm{NaCl}$ plates. Exact mass was determined using electrospray ionization.

Scheme 1. Preparation of substrate 1 (precursor of lactone 2; Table 1)

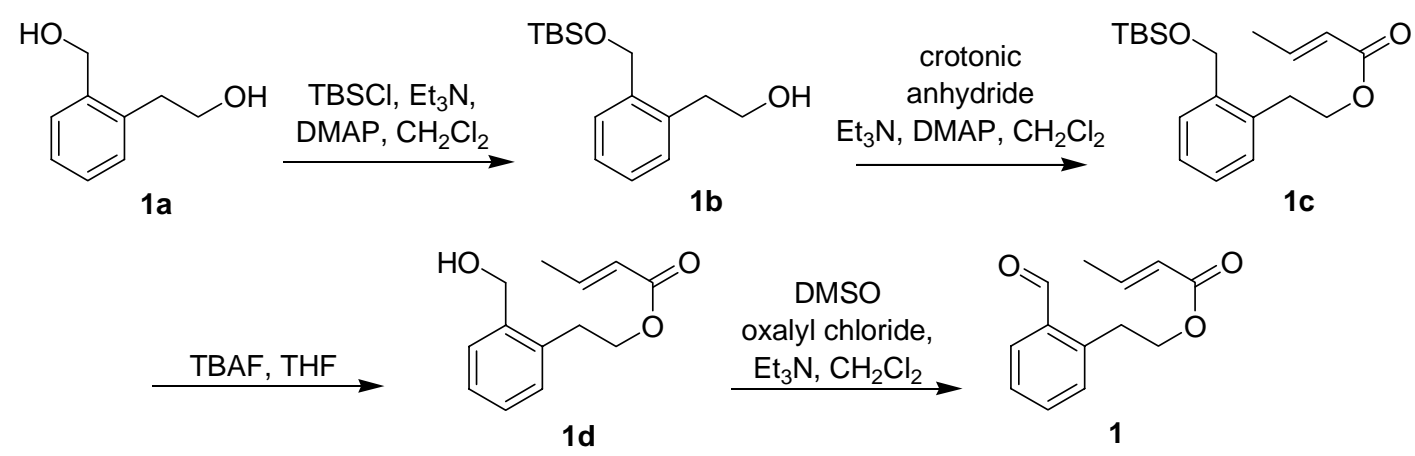

The monoprotection of the readily available diol $\mathbf{1 a}^{1}$ with $\mathrm{TBSCl}$ provided alcohol 1b, which was acylated with crotonic anhydride to obtain ester 1c. Treatment of 1c with TBAF to liberate the alcohol 1d followed by a Swern oxidation resulted in aldehyde 1.

\section{2-[2-(tert-Butyldimethylsilanyloxymethyl)phenyl]ethanol (1b):}

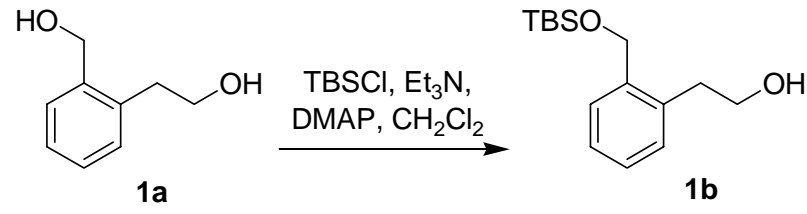

A solution of TBSCl $(13.1 \mathrm{~g}, 87 \mathrm{mmol})$ in $\mathrm{CH}_{2} \mathrm{Cl}_{2}(150 \mathrm{~mL})$ was added dropwise over 4 hours to a stirred solution of diol $\mathbf{1 a}^{1}(13.2 \mathrm{~g}, 87 \mathrm{mmol}), \mathrm{Et}_{3} \mathrm{~N}(15.5 \mathrm{~mL}, 104$ mmol), and DMAP (1.06 g, $8.7 \mathrm{mmol})$ in $\mathrm{CH}_{2} \mathrm{Cl}_{2}(150 \mathrm{~mL})$ at room temperature. ${ }^{2}$ The reaction was allowed to stir overnight (14 hours), after which time $\mathrm{Et}_{2} \mathrm{O}(400 \mathrm{~mL})$ was added. The solution was washed with saturated $\mathrm{NaHCO}_{3}$ and brine, dried over anhydrous $\mathrm{MgSO}_{4}$, and concentrated under reduced pressure on the rotary evaporator. The crude product was purified by flash chromatography (10:1 hexanes:EtOAc) to provide the desired alcohol 1b $(2.83 \mathrm{~g}, 7.9 \mathrm{mmol}, 12 \%)$ and the regioisomer $(6.47 \mathrm{~g}, 24.2 \mathrm{mmol}$, $28 \%)$.

${ }^{1} \mathrm{H}$ NMR $\left(400 \mathrm{MHz} ; \mathrm{CDCl}_{3}\right): \delta$ 7.36-7.32 (m, 1H), 7.27-7.18 (m, 3H), $4.73(\mathrm{~s}$, $2 \mathrm{H}), 3.85(\mathrm{t}, 2 \mathrm{H}, \mathrm{J}=6.4 \mathrm{~Hz}), 2.91(\mathrm{t}, 2 \mathrm{H}, \mathrm{J}=6.4 \mathrm{~Hz}), 0.92(\mathrm{~s}, 9 \mathrm{H}), 0.11(\mathrm{~s}, 6 \mathrm{H}) .{ }^{13} \mathrm{C}$ NMR $\left(100 \mathrm{MHz} ; \mathrm{CDCl}_{3}\right): \delta 139.1,137.2,130.1,128.6,128.1,126.8,63.8,63.5,35.6$, 26.2, 18.7, -5.0. LRMS (ESI) $\mathrm{m} / \mathrm{z}$ calc'd for $\mathrm{C}_{15} \mathrm{H}_{26} \mathrm{O}_{2} \mathrm{SiNa}[\mathrm{M}+\mathrm{Na}]^{+}$: 289.2; found: 289.3

\footnotetext{
${ }^{1}$ Warnell, J. L.; Shriner, R. L. J. Am. Chem. Soc. 1957, 79, 3165.

${ }^{2}$ Followed procedure in reference: Xiao, X-Y.; Nova, M. P.; Czarnik, A. W. J. Comb. Chem. 1999, 1, 379.
} 
But-2-enoic acid 2-[2-(tert-Butyldimethylsilanyloxymethyl)phenyl]ethyl ester (1c):

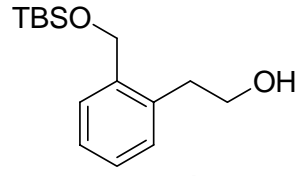

$1 b$

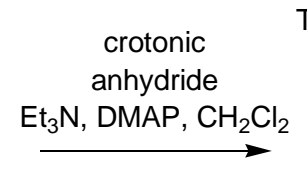

$\underbrace{C B S O}_{1 c}$

A solution of $\mathrm{Et}_{3} \mathrm{~N}(7.39 \mathrm{~mL}, 53.0 \mathrm{mmol})$ and DMAP $(0.26 \mathrm{~g}, 2.1 \mathrm{mmol})$ in $\mathrm{CH}_{2} \mathrm{Cl}_{2}(50 \mathrm{~mL})$ was added to a stirred solution of alcohol $\mathbf{1 b}(2.82 \mathrm{~g}, 10.6 \mathrm{mmol})$ and crotonic anhydride $(2.35 \mathrm{~mL}, 16.1 \mathrm{mmol})$ in $\mathrm{CH}_{2} \mathrm{Cl}_{2}(50 \mathrm{~mL})$ at $0^{\circ} \mathrm{C}$. After 4 hours at $0^{\circ} \mathrm{C}$, the reaction was concentrated under reduced pressure on a rotary evaporator. EtOAc $(50 \mathrm{~mL})$ was added, and the organic solution was washed with saturated $\mathrm{NaHCO}_{3}$, deionized water, and brine. The organic phase was dried over anhydrous $\mathrm{MgSO}_{4}$ and concentrated under reduced pressure on the rotary evaporator. The crude product was purified by flash chromatography (10:1 hexanes:EtOAc) to provide ester 1c $(2.98 \mathrm{~g}, 8.9$ mmol, 84\%).

${ }^{1} \mathrm{H}$ NMR $\left(400 \mathrm{MHz} ; \mathrm{CDCl}_{3}\right): \delta 7.42-7.38(\mathrm{~m}, 1 \mathrm{H}), 7.23-7.16(\mathrm{~m}, 3 \mathrm{H}), 6.95(\mathrm{dq}$, $1 \mathrm{H}, \mathrm{J}=7.0,15.5 \mathrm{~Hz}), 5.82(\mathrm{dq}, 1 \mathrm{H}, \mathrm{J}=1.7,15.5 \mathrm{~Hz}), 4.75(\mathrm{~s}, 2 \mathrm{H}), 4.31(\mathrm{t}, 2 \mathrm{H}, \mathrm{J}=7.2$ $\mathrm{Hz}), 2.96(\mathrm{t}, 2 \mathrm{H}, \mathrm{J}=7.2 \mathrm{~Hz}), 1.86(\mathrm{dd}, 3 \mathrm{H}, \mathrm{J}=1.7,7.1 \mathrm{~Hz}), 0.92(\mathrm{~s}, 9 \mathrm{H}), 0.09(\mathrm{~s}, 6 \mathrm{H})$. ${ }^{13} \mathrm{C}$ NMR $\left(100 \mathrm{MHz} ; \mathrm{CDCl}_{3}\right): \delta 166.6,145.0,139.5,135.4,129.8,127.7,127.5,126.9$, $122.9,64.5,63.4,31.6,26.2,18.6,18.2,-5.1$. LRMS (ESI) $\mathrm{m} / \mathrm{z}$ calc'd for $\mathrm{C}_{19} \mathrm{H}_{30} \mathrm{O}_{3} \mathrm{SiNa}$ $[\mathrm{M}+\mathrm{Na}]^{+}:$357.2; found: 357.4 .

\section{But-2-enoic acid 2-(2-hydroxymethylphenyl)ethyl ester (1d):}

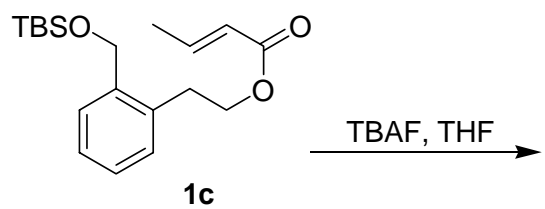

1c<smiles>C/C=C\C(=O)OCCc1ccccc1CO</smiles>

1d

TBAF (12.3 mL, $12.3 \mathrm{mmol}, 1 \mathrm{M}$ solution in THF) was added to a stirred solution of $1 \mathrm{c}(2.74 \mathrm{~g}, 8.2 \mathrm{mmol})$ in THF $(80 \mathrm{~mL})$ at $0^{\circ} \mathrm{C}$. After 45 minutes at $0^{\circ} \mathrm{C}$, the reaction was quenched with saturated $\mathrm{NH}_{4} \mathrm{Cl}(20 \mathrm{~mL})$. The organic phase was separated, washed with deionized water and brine, dried over anhydrous $\mathrm{MgSO}_{4}$, and concentrated under reduced pressure on the rotary evaporator. The crude product was purified by flash chromatography (1:1 hexanes:EtOAc) to provide alcohol 1d (1.78 g, $8.1 \mathrm{mmol}, 99 \%)$.

${ }^{1} \mathrm{H}$ NMR $\left(400 \mathrm{MHz} ; \mathrm{CDCl}_{3}\right): \delta$ 7.37-7.33 (m, 1H), 7.28-7.19 (m, 3H), $6.95(\mathrm{dq}$, $1 \mathrm{H}, \mathrm{J}=6.9,15.5 \mathrm{~Hz}), 5.80(\mathrm{dq}, 1 \mathrm{H}, \mathrm{J}=1.7,15.5 \mathrm{~Hz}), 4.72(\mathrm{~s}, 2 \mathrm{H}), 4.33(\mathrm{t}, 2 \mathrm{H}, \mathrm{J}=7.2$ $\mathrm{Hz}), 3.04(\mathrm{t}, 2 \mathrm{H}, \mathrm{J}=7.2 \mathrm{~Hz}), 2.18$ (br s, $1 \mathrm{H}), 1.85$ (dd, 3H, J = 1.7, 7.0 Hz). ${ }^{13} \mathrm{C} \mathrm{NMR}$ $\left(100 \mathrm{MHz} ; \mathrm{CDCl}_{3}\right): \delta 166.8,145.4,139.3,136.3,130.4,129.3,128.4,127.3,122.6,64.9$, 63.6, 31.9, 18.2. LRMS (ESI) $\mathrm{m} / \mathrm{z}$ calc'd for $\mathrm{C}_{13} \mathrm{H}_{16} \mathrm{O}_{3} \mathrm{Na}[\mathrm{M}+\mathrm{Na}]^{+}$: 243.1; found: 243.2. 
But-2-enoic acid 2-(2-formylphenyl)ethyl ester (1):
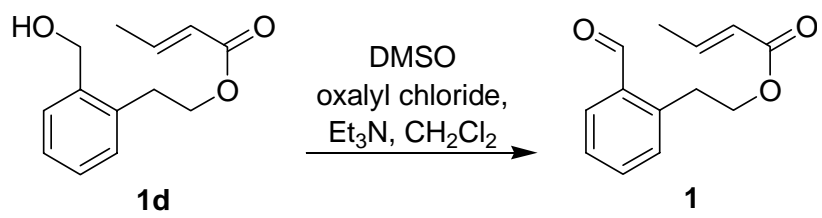

Oxalyl chloride $(0.97 \mathrm{~mL}, 11.1 \mathrm{mmol})$ was added dropwise to a stirred solution of DMSO $(2.11 \mathrm{~mL}, 29.6 \mathrm{mmol})$ in $\mathrm{CH}_{2} \mathrm{Cl}_{2}(100 \mathrm{~mL})$ at $-78^{\circ} \mathrm{C}$. After 1 hour, a solution of alcohol 1d (1.64 g, $7.4 \mathrm{mmol})$ in $\mathrm{CH}_{2} \mathrm{Cl}_{2}(10 \mathrm{~mL})$ was added via canula. After 30 minutes, $\mathrm{Et}_{3} \mathrm{~N}(4.20 \mathrm{~mL}, 29.6 \mathrm{mmol})$ was added by syringe. After an additional 30 minutes, the dry ice/acetone bath was removed, and the reaction was allowed to warm to room temperature. The reaction was quenched with deionized water $(100 \mathrm{~mL})$. The organic layer was separated and washed with saturated $\mathrm{NaHCO}_{3}$, brine, dried over anhydrous $\mathrm{MgSO}_{4}$, and concentrated under reduced pressure on the rotary evaporator. The crude product was purified by flash chromatography (8:1 hexances:EtOAc) to afford aldehyde $\mathbf{1}(1.26 \mathrm{~g}, 5.8 \mathrm{mmol}, 78 \%)$.

${ }^{1} \mathrm{H}$ NMR $\left(400 \mathrm{MHz} ; \mathrm{CDCl}_{3}\right): \delta 10.20(\mathrm{~s}, 1 \mathrm{H}), 7.80(\mathrm{dd}, 1 \mathrm{H}, \mathrm{J}=1.6,7.7 \mathrm{~Hz}), 7.50$ $(\mathrm{dt}, 1 \mathrm{H}, \mathrm{J}=1.5,7.4 \mathrm{~Hz}), 7.40(\mathrm{dt}, 1 \mathrm{H}, \mathrm{J}=1.3,7.5 \mathrm{~Hz}), 7.30-7.27(\mathrm{~m}, 1 \mathrm{H}), 6.89(\mathrm{dq}, 1 \mathrm{H}, \mathrm{J}$ $=6.9,15.6 \mathrm{~Hz}), 5.76(\mathrm{dq}, 1 \mathrm{H}, \mathrm{J}=1.7,15.6 \mathrm{~Hz}), 4.33(\mathrm{t}, 2 \mathrm{H}, \mathrm{J}=6.7 \mathrm{~Hz}), 3.37(\mathrm{t}, 2 \mathrm{H}, \mathrm{J}=$ $6.7 \mathrm{~Hz}), 1.82(\mathrm{dd}, 3 \mathrm{H}, \mathrm{J}=1.7,7.0 \mathrm{~Hz}) .{ }^{13} \mathrm{C} \mathrm{NMR}\left(100 \mathrm{MHz} ; \mathrm{CDCl}_{3}\right): \delta 192.9,166.5$, $145.1,140.5,134.4,133.9,133.2,132.0,127.5,122.6,64.4,32.1,18.2$. IR $\left(\mathrm{cm}^{-1}\right): 2971$, $1720,1698,1657,1294,1268,1183,758$. HRMS (ESI) $\mathrm{m} / \mathrm{z}$ calc'd for $\mathrm{C}_{13} \mathrm{H}_{15} \mathrm{O}_{3}$ $[\mathrm{M}+\mathrm{H}]^{+}:$219.1014; found: 219.1015 .

Scheme 2. Preparation of substrate $\mathbf{3 f}$ (precursor of lactone 3; Table 2, entry 1)
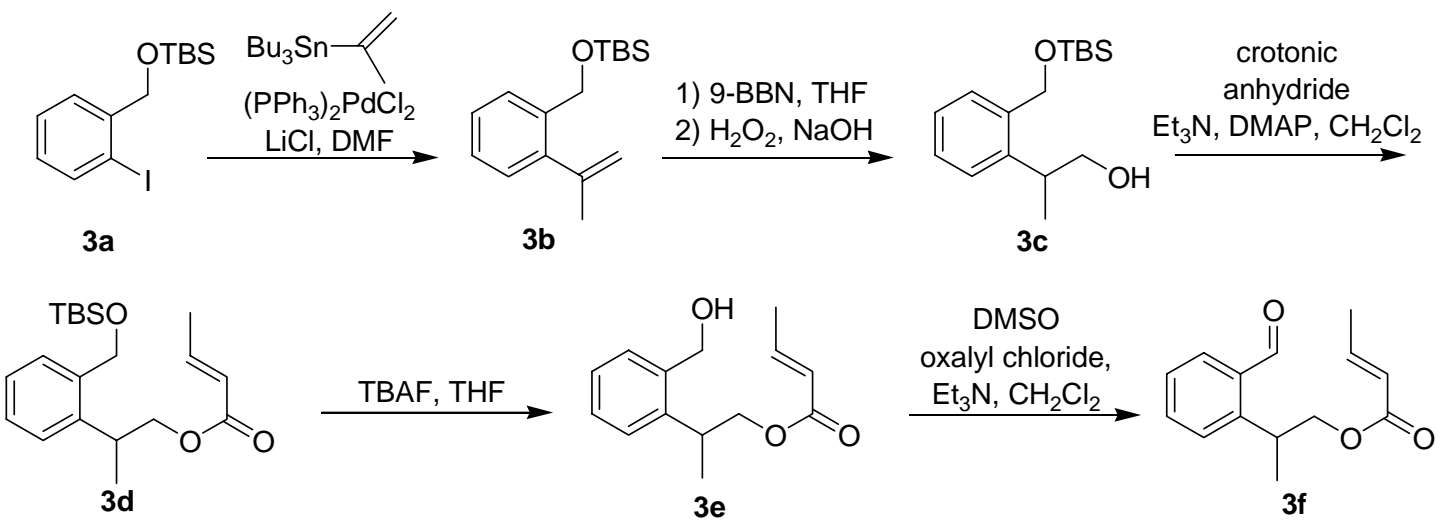

A Stille coupling of TBS-ether $3 \mathbf{a}^{3}$ with tributyl-(2-propenyl)-stannane provided alkene $3 \mathbf{b}$, which was subjected to a hydroboration-oxidation with $9-\mathrm{BBN} / \mathrm{H}_{2} \mathrm{O}_{2} / \mathrm{NaOH}$ to obtain alcohol 3c. Acylation of 3c with crotonic anhydride afforded ester 3d. Deprotection of the TBS group with TBAF followed by oxidation under the Swern conditions provided aldehyde $\mathbf{3 f}$.

\footnotetext{
${ }^{3}$ Lautens, M.; Paquin, J-F.; Piguel, S.; Dahlmann, M. J. Org. Chem. 2001, 66, 8127.
} 
tert-Butyl-(2-isopropenylbenzyloxy)dimethylsilane (3b):

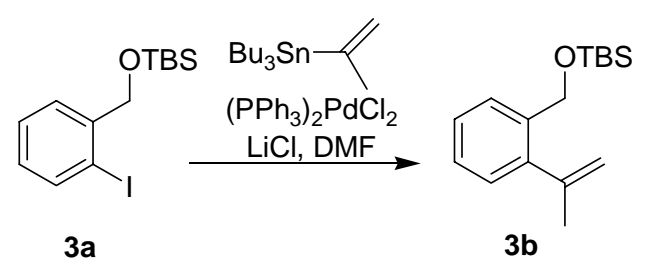

TBS ether $3 \mathrm{a}^{3}$ (5.13g, $\left.17.0 \mathrm{mmol}\right)$, 2-(tributylstannyl)propene $(6.20 \mathrm{~g}, 18.7 \mathrm{mmol})$, anhydrous DMF $(100 \mathrm{~mL})$, dry $\mathrm{LiCl}(3.2 \mathrm{~g}, 54.5 \mathrm{mmol})$, and bis(triphenylphosphine)palladium(II) chloride $(0.24 \mathrm{~g}, \quad 0.34 \mathrm{mmol})$ were added sequentially to a flask equipped with a reflux condenser and a stir bar. The mixture was heated to $110^{\circ} \mathrm{C}$ and allowed to stir at this temperature for 4 days. The cooled reaction mixture was passed through a pad of Celite. After washing the pad with EtOAc (100 $\mathrm{mL})$, the filtrate was diluted with hexanes $(300 \mathrm{~mL})$ resulting in a biphasic solution. The layers were separated, and the DMF-containing phase was extracted once more with hexanes $(100 \mathrm{~mL})$. The combined hexanes/EtOAc extractes were washed with deionized water, 1.0 $\mathrm{M} \mathrm{CuSO}_{4}$, brine, then dried over anhydrous $\mathrm{MgSO}_{4}$, and concentrated under reduced pressure on a rotary evaporator. The crude product was purified by flash chromatography (30:1 hexanes:EtOAc) to provide $\mathbf{3 b}$ as a colorless oil $(3.30 \mathrm{~g}, 12.6$ mmol, 74\%) .

${ }^{1} \mathrm{H}$ NMR $\left(400 \mathrm{MHz} ; \mathrm{CDCl}_{3}\right): \delta 7.57-7.49(\mathrm{~m}, 1 \mathrm{H}), 7.26(\mathrm{dt}, 1 \mathrm{H}, \mathrm{J}=1.6,7.4 \mathrm{~Hz})$, $7.20(\mathrm{dt}, 1 \mathrm{H}, \mathrm{J}=1.6,7.5 \mathrm{~Hz}), 7.10(\mathrm{dd}, 1 \mathrm{H}, \mathrm{J}=1.4,7.4 \mathrm{~Hz}), 5.19-5.16(\mathrm{~m}, 1 \mathrm{H}), 4.83-4.81$ $(\mathrm{m}, 1 \mathrm{H}), 4.72(\mathrm{~s}, 2 \mathrm{H}), 2.03-2.01(\mathrm{~m}, 3 \mathrm{H}), 0.92(\mathrm{~s}, 9 \mathrm{H}), 0.08(\mathrm{~s}, 6 \mathrm{H}) .{ }^{13} \mathrm{C}$ NMR $(100$ $\left.\mathrm{MHz} ; \mathrm{CDCl}_{3}\right): \delta 144.6,142.2,137.9,127.7,127.2,127.1,128.8,115.3,62.9,26.2,25.0$, 18.6, -5.1. LRMS (ESI) m/z calc'd for $\mathrm{C}_{16} \mathrm{H}_{27} \mathrm{OSi}[\mathrm{M}+\mathrm{H}]^{+}$: 263.2; found: 263.3.

\section{2-[2-(tert-Butyldimethylsilanyloxymethyl)phenyl]propan-1-ol (3c):}
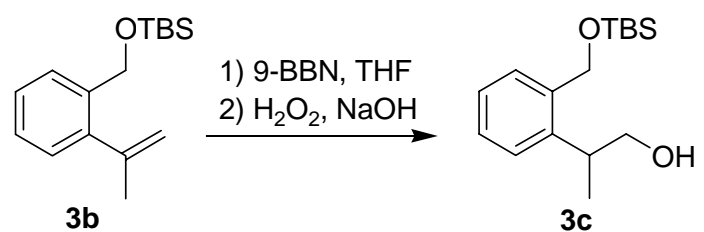

9-BBN (31.5 mL, $15.8 \mathrm{mmol}, 0.5 \mathrm{M}$ solution in THF) was slowly added to a stirred solution of $3 \mathbf{b}(3.30 \mathrm{~g}, 12.6 \mathrm{mmol})$ in THF $(50 \mathrm{~mL})$. The solution was heated to reflux overnight (18 hours), and then cooled to $0^{\circ} \mathrm{C}$. EtOH $(18 \mathrm{~mL}), \mathrm{NaOH}(18 \mathrm{~mL}, 2 \mathrm{M}$ solution in water), and $\mathrm{H}_{2} \mathrm{O}_{2}(15 \mathrm{~mL}, 30 \%$ solution $)$ were added sequentially at a rate slow enough to prevent exotherm. The mixture was heated to reflux ( 1 hour), cooled to $0^{\circ} \mathrm{C}$, and quenched with saturated $\mathrm{Na}_{2} \mathrm{CO}_{3}(25 \mathrm{~mL})$. After extracting the aqueous phase with $\mathrm{Et}_{2} \mathrm{O}$, the organic phases were combined, dried over anhydrous $\mathrm{MgSO}_{4}$, and concentrated under reduced pressure on the rotary evaporator. The crude product was purified by flash chromatography (10:1 hexanes:EtOAc) to provide the desired alcohol 3c (2.22 g, $7.9 \mathrm{mmol}, 63 \%)$.

${ }^{1} \mathrm{H}$ NMR (400 MHz; $\left.\mathrm{CDCl}_{3}\right): \delta 7.34-7.17(\mathrm{~m}, 4 \mathrm{H}), 4.81(\mathrm{~d}, 1 \mathrm{H}, \mathrm{J}=12.2 \mathrm{~Hz})$, $4.67(\mathrm{~d}, 1 \mathrm{H}, \mathrm{J}=12.2 \mathrm{~Hz}), 3.75(\mathrm{dd}, 1 \mathrm{H}, \mathrm{J}=5.9,10.4 \mathrm{~Hz}), 3.62(\mathrm{dd}, 1 \mathrm{H}, \mathrm{J}=8.6,10.3 \mathrm{~Hz})$, 3.52-3.42 (m, 1H), $1.24(\mathrm{~d}, 1 \mathrm{H}, \mathrm{J}=7.0 \mathrm{~Hz}), 0.93(\mathrm{~s}, 9 \mathrm{H}), 0.12(\mathrm{~s}, 3 \mathrm{H}), 0.11(\mathrm{~s}, 3 \mathrm{H}) .{ }^{13} \mathrm{C}$ 
NMR (100 MHz; $\left.\mathrm{CDCl}_{3}\right): \delta 142.7,138.8,128.6,128.5,126.7,126.0,69.0,64.1,36.8$, 26.2, 18.6, 18.1, -5.0, -5.1. LRMS (ESI) $\mathrm{m} / \mathrm{z}$ calc'd for $\mathrm{C}_{16} \mathrm{H}_{28} \mathrm{O}_{2} \mathrm{SiNa}[\mathrm{M}+\mathrm{Na}]^{+}: 303.2$; found: 303.3 .

But-2-enoic acid 2-[2-(tert-Butyldimethylsilanyloxymethyl)phenyl]propyl ester (3d):

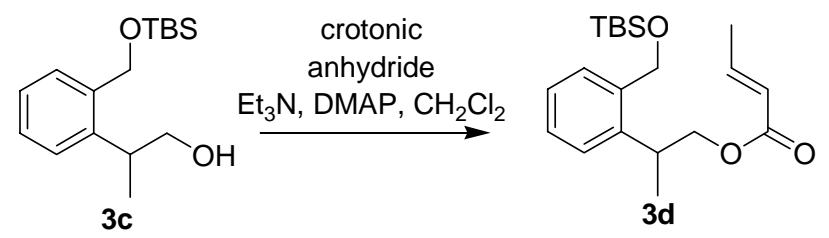

Compound 3d was prepared from $\mathbf{3 c}$ by following the procedure for the preparation of 1c and was purified by flash chromatography (10:1 hexanes:EtOAc) to provide a colorless oil $(2.64 \mathrm{~g}, 7.6 \mathrm{mmol}, 96 \%)$.

${ }^{1} \mathrm{H}$ NMR $\left(500 \mathrm{MHz} ; \mathrm{CDCl}_{3}\right): \delta$ 7.40-7.36 (m, 1H), 7.26-7.17 (m, 3H), $6.91(\mathrm{dq}$, $1 \mathrm{H}, \mathrm{J}=7.1,15.5 \mathrm{~Hz}), 5.79(\mathrm{dq}, 1 \mathrm{H}, \mathrm{J}=1.7,15.6 \mathrm{~Hz}), 4.79(\mathrm{~d}, 1 \mathrm{H}, \mathrm{J}=12.8 \mathrm{~Hz}), 4.76(\mathrm{~d}$, $1 \mathrm{H}, \mathrm{J}=12.8 \mathrm{~Hz}$ ), 4.22-4.15 (m, 2H), $3.39(\mathrm{~h}, 1 \mathrm{H}, \mathrm{J}=7.1 \mathrm{~Hz}), 1.85(\mathrm{dd}, 3 \mathrm{H}, \mathrm{J}=1.7,6.8$ $\mathrm{Hz}), 1.27(\mathrm{~d}, 3 \mathrm{H}, \mathrm{J}=6.9 \mathrm{~Hz}), 0.91(\mathrm{~s}, 9 \mathrm{H}), 0.08(\mathrm{~s}, 3 \mathrm{H}), 0.07(\mathrm{~s}, 3 \mathrm{H}) .{ }^{13} \mathrm{C}$ NMR $(100$ $\left.\mathrm{MHz} ; \mathrm{CDCl}_{3}\right): \delta 166.6,144.8,141.2,138.8,127.8,127.7,126.6,126.0,122.8,69.2$, $63.5,33.4,26.1,18.5,18.4,18.2,-5.0,-5.1$. LRMS (ESI) $\mathrm{m} / \mathrm{z}$ calc'd for $\mathrm{C}_{20} \mathrm{H}_{32} \mathrm{O}_{3} \mathrm{SiNa}$ $[\mathrm{M}+\mathrm{Na}]^{+}: 371.2$; found: 371.4 .

\section{But-2-enoic acid 2-(2-hydroxymethylphenyl)propyl ester (3e):}

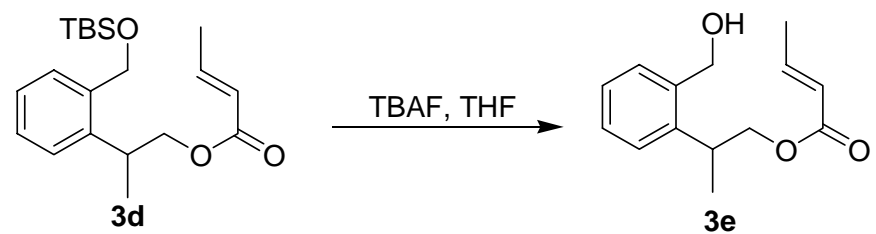

Compound 3e was prepared from $\mathbf{3 d}$ by following the procedure for the preparation of 1d and was purified by flash chromatography (2:1 hexanes:EtOAc) to provide a colorless oil $(1.49 \mathrm{~g}, 6.4 \mathrm{mmol}, 84 \%)$.

${ }^{1} \mathrm{H}$ NMR $\left(400 \mathrm{MHz} ; \mathrm{CDCl}_{3}\right): \delta 7.35-7.17(\mathrm{~m}, 4 \mathrm{H}), 6.92(\mathrm{dq}, 1 \mathrm{H}, \mathrm{J}=6.9,15.6$ $\mathrm{Hz}), 5.78(\mathrm{dq}, 1 \mathrm{H}, \mathrm{J}=1.7,15.6 \mathrm{~Hz}), 4.77(\mathrm{~d}, 1 \mathrm{H}, \mathrm{J}=12.4 \mathrm{~Hz}), 4.66(\mathrm{~d}, 1 \mathrm{H}, \mathrm{J}=12.3 \mathrm{~Hz})$, $4.27(\mathrm{dd}, 1 \mathrm{H}, \mathrm{J}=6.5,10.8 \mathrm{~Hz}), 4.09(\mathrm{dd}, 1 \mathrm{H}, \mathrm{J}=7.8,10.8 \mathrm{~Hz}), 3.49(\mathrm{~h}, 1 \mathrm{H}, \mathrm{J}=6.9 \mathrm{~Hz})$, 2.29 (br s, $1 \mathrm{H}), 1.83(\mathrm{dd}, 3 \mathrm{H}, \mathrm{J}=1.7,6.9 \mathrm{~Hz}), 1.31(\mathrm{~d}, 3 \mathrm{H}, \mathrm{J}=7.0 \mathrm{~Hz}) .{ }^{13} \mathrm{C}$ NMR $(100$ $\left.\mathrm{MHz} ; \mathrm{CDCl}_{3}\right): \delta 166.8,145.5,141.8,138.7,129.4,128.6,127.0,126.4,122.5,69.8$, 63.6, 33.7, 18.4, 18.2. LRMS (ESI) $\mathrm{m} / \mathrm{z}$ calc'd for $\mathrm{C}_{14} \mathrm{H}_{18} \mathrm{O}_{3} \mathrm{Na}[\mathrm{M}+\mathrm{Na}]^{+}:$: 257.1; found: 257.2.

\section{But-2-enoic acid 2-(2-formylphenyl)propyl ester (3f):}
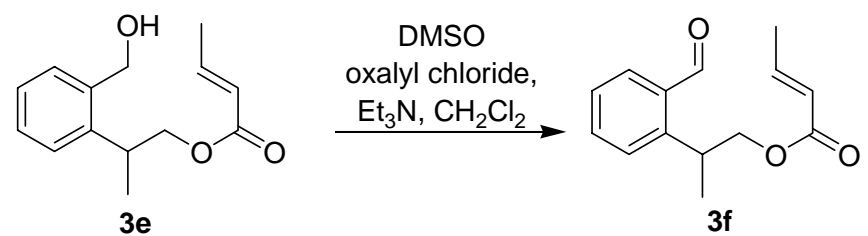
Compound 3f was prepared from $3 \mathbf{e}$ by following the procedure for the preparation of $\mathbf{1}$ and was purified by flash chromatography (5:1 hexanes:EtOAc) to provide a colorless oil $(1.13 \mathrm{~g}, 4.9 \mathrm{mmol}, 77 \%)$.

${ }^{1} \mathrm{H}$ NMR $\left(400 \mathrm{MHz} ; \mathrm{CDCl}_{3}\right): \delta 10.30(\mathrm{~s}, 1 \mathrm{H}), 7.82(\mathrm{dd}, 1 \mathrm{H}, \mathrm{J}=1.5,7.7 \mathrm{~Hz}), 7.56$ $(\mathrm{dt}, 1 \mathrm{H}, \mathrm{J}=1.5,7.6 \mathrm{~Hz}), 7.45-7.41(\mathrm{~m}, 1 \mathrm{H}), 7.39(\mathrm{dt}, 1 \mathrm{H}, \mathrm{J}=1.2,7.5 \mathrm{~Hz}), 6.88(\mathrm{dq}, 1 \mathrm{H}, \mathrm{J}$ $=6.9,15.6 \mathrm{~Hz}), 5.75(\mathrm{dq}, 1 \mathrm{H}, \mathrm{J}=1.7,15.6 \mathrm{~Hz}), 4.32-4.22(\mathrm{~m}, 3 \mathrm{H}), 1.82(\mathrm{dd}, 3 \mathrm{H}, \mathrm{J}=1.7$, $7.0 \mathrm{~Hz}), 1.36-1.33$ (m, 3H). ${ }^{13} \mathrm{C}$ NMR $\left(100 \mathrm{MHz} ; \mathrm{CDCl}_{3}\right): \delta$ 192.7, 166.6, 146.0, 145.2, 134.2, 134.1, 132.6, 127.2, 127.1, 122.6, 68.7, 32.4, 18.2, 18.1. IR ( $\left.\mathrm{cm}^{-1}\right): 2973,2738$, $1719,1691,1658,1182,1023,765$. HRMS (ESI) $m / z$ calc'd for $\mathrm{C}_{14} \mathrm{H}_{17} \mathrm{O}_{3}[\mathrm{M}+\mathrm{H}]^{+}$: 233.1176; found: 233.1172 .

Scheme 3. Preparation of substrate 4e (precursor of lactone 4; Table 2, entry 2)

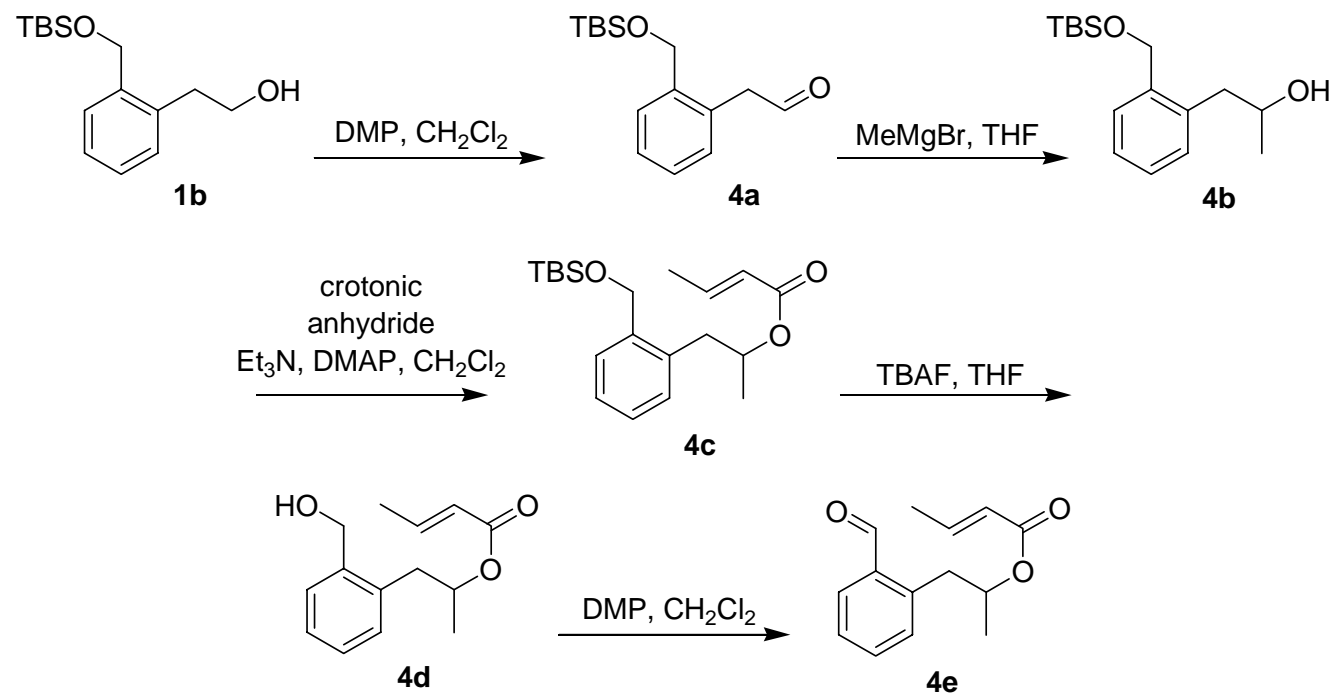

Oxidation of $\mathbf{1 b}$ with Dess-Martin periodinane (DMP) ${ }^{4}$ provided aldehyde $\mathbf{4 a}$, which was treated with methylmagnesium bromide to obtain alcohol $\mathbf{4 b}$. Esterification of 4b with crotonic anhydride followed by a TBS deprotection resulted in 4d. Conversion of the alcohol 4d to aldehyde 4e was achieved with DMP.

\section{[2-(tert-Butyldimethylsilanyloxymethyl)phenyl]acetaldehyde (4a):}

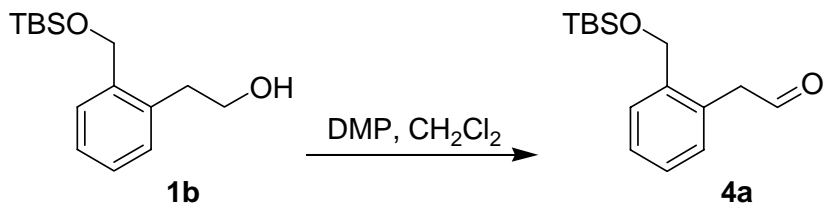

$\operatorname{DMP}^{4}$ (3.5 g, $\left.8.3 \mathrm{mmol}\right)$ was added to a stirred solution of alcohol $\mathbf{1 b}$ (1.38 g, 5.2 mmol) in $\mathrm{CH}_{2} \mathrm{Cl}_{2}(50 \mathrm{~mL})$. The mixture was stirred overnight (14 hours) at room temperature. The reaction was diluted with $\mathrm{Et}_{2} \mathrm{O}(50 \mathrm{~mL})$, then saturated $\mathrm{NaHCO}_{3} / \mathrm{Na}_{2} \mathrm{~S}_{2} \mathrm{O}_{3}$ (25 mL, 1/1 mixture) was added, and the reaction was stirred vigorously for 15 minutes. After the mixture was diluted further with $\mathrm{Et}_{2} \mathrm{O}(150 \mathrm{~mL})$, the organic phase was separated and washed with saturated $\mathrm{NaHCO}_{3}$, deionized water, brine,

\footnotetext{
${ }^{4}$ DMP was prepared following: Frigerio, M.; Santagostino, M.; Sputore, S. J. Org. Chem. 1999, 64, 4537.
} 
dried over anhydrous $\mathrm{MgSO}_{4}$, and concentrated under reduced pressure on the rotary evaporator. The crude product was purified by flash chromatography (10:1 hexanes:EtOAc) yielding aldehyde $4 a(1.27 \mathrm{~g}, 4.8 \mathrm{mmol}, 92 \%)$.

${ }^{1} \mathrm{H}$ NMR $\left(400 \mathrm{MHz} ; \mathrm{CDCl}_{3}\right): \delta 9.69(\mathrm{t}, 1 \mathrm{H}, \mathrm{J}=2.1 \mathrm{~Hz}), 7.40-7.14(\mathrm{~m}, 4 \mathrm{H}), 4.67$ $(\mathrm{s}, 2 \mathrm{H}), 3.74(\mathrm{~d}, 2 \mathrm{H}, \mathrm{J}=2.1 \mathrm{~Hz}), 0.90(\mathrm{~s}, 9 \mathrm{H}), 0.07(\mathrm{~s}, 6 \mathrm{H}) .{ }^{13} \mathrm{C}$ NMR $(100 \mathrm{MHz}$; $\left.\mathrm{CDCl}_{3}\right): \delta 199.9,140.0,131.1,130.8,128.5,128.2,127.9,64.1,47.9,26.1,18.6,-5.1$. LRMS (ESI) $\mathrm{m} / \mathrm{z}$ calc'd for $\mathrm{C}_{15} \mathrm{H}_{25} \mathrm{O}_{2} \mathrm{Si}[\mathrm{M}+\mathrm{H}]^{+}$: 265.2; found: 265.3.

1-[2-(tert-Butyldimethylsilanyloxymethyl)phenyl]propan-2-ol (4b):

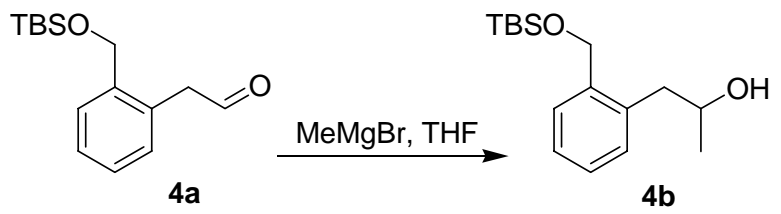

$\mathrm{MeMgBr}\left(1.76 \mathrm{~mL}, 5.28 \mathrm{mmol}, 3.0 \mathrm{M}\right.$ solution in $\left.\mathrm{Et}_{2} \mathrm{O}\right)$ was added to a stirred solution of aldehyde $4 a(1.27 \mathrm{~g}, 4.8 \mathrm{mmol})$ in THF $(50 \mathrm{~mL})$ at $0^{\circ} \mathrm{C}$. After 1 hour, the reaction was quenched with saturated $\mathrm{NH}_{4} \mathrm{Cl}(25 \mathrm{~mL})$. The organic phase was separated, washed with deionized water and brine, dried over anhydrous $\mathrm{MgSO}_{4}$, and concentrated under reduced pressure on the rotary evaporator. The crude product was purified by flash chromatography (5:1 hexanes:EtOAc) to provide alcohol $\mathbf{4 b}(1.27 \mathrm{~g}, 4.6 \mathrm{mmol}, 96 \%)$.

${ }^{1} \mathrm{H}$ NMR $\left(400 \mathrm{MHz} ; \mathrm{CDCl}_{3}\right): \delta$ 7.36-7.33 $(\mathrm{m}, 1 \mathrm{H}), 7.27-7.17(\mathrm{~m}, 3 \mathrm{H}), 4.77(\mathrm{~d}$, $1 \mathrm{H}, \mathrm{J}=12.4 \mathrm{~Hz}$ ), $4.67(\mathrm{~d}, 1 \mathrm{H}, \mathrm{J}=12.4 \mathrm{~Hz}), 4.00(\mathrm{ddq}, 1 \mathrm{H}, \mathrm{J}=4.3,6.2,8.6 \mathrm{~Hz}$ ), 2.82 (dd, $1 \mathrm{H}, \mathrm{J}=4.1,13.8 \mathrm{~Hz}$ ), $2.72(\mathrm{dd}, 1 \mathrm{H}, \mathrm{J}=8.5,13.8 \mathrm{~Hz}$ ), 2.39 (br s, 1H), 1.27 (d, 3H, J = 6.1 $\mathrm{Hz}), 0.92(\mathrm{~s}, 9 \mathrm{H}), 0.12(\mathrm{~s}, 3 \mathrm{H}), 0.10(\mathrm{~s}, 3 \mathrm{H}) .{ }^{13} \mathrm{C} \mathrm{NMR}\left(100 \mathrm{MHz} ; \mathrm{CDCl}_{3}\right): \delta$ 139.1, $137.2,130.5,128.7,128.0,126.8,69.0,63.9,42.1,26.2,23.9,18.7,-5.0$. LRMS (ESI) $\mathrm{m} / \mathrm{z}$ calc'd for $\mathrm{C}_{16} \mathrm{H}_{28} \mathrm{O}_{2} \mathrm{SiNa}[\mathrm{M}+\mathrm{Na}]^{+}:$303.2; found: 303.3.

\section{But-2-enoic acid 2-[2-(tert-Butyldimethylsilanyloxymethyl)phenyl]-1-methylethyl} ester (4c):
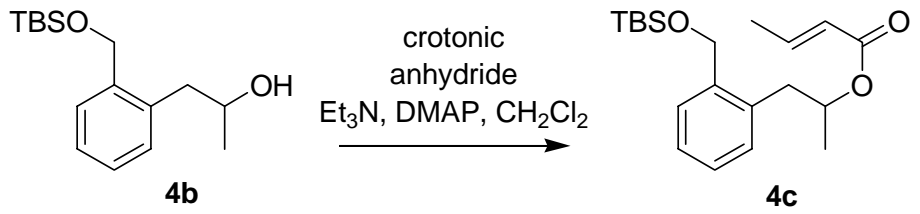

Compound 4c was prepared from $\mathbf{4 b}$ by following the procedure for the preparation of 1c and was purified by flash chromatography (5:1 hexanes:EtOAc) to provide a colorless oil $(1.08 \mathrm{~g}, 3.1 \mathrm{mmol}, 67 \%)$.

${ }^{1} \mathrm{H}$ NMR $\left(400 \mathrm{MHz} ; \mathrm{CDCl}_{3}\right): \delta$ 7.44-7.40 (m, 1H), 7.23-7.13 (m, 3H), $6.91(\mathrm{dq}$, $1 \mathrm{H}, \mathrm{J}=7.0,15.5 \mathrm{~Hz}), 5.78(\mathrm{dq}, 1 \mathrm{H}, \mathrm{J}=1.7,15.5 \mathrm{~Hz}), 5.16(\mathrm{~h}, 1 \mathrm{H}, \mathrm{J}=6.3 \mathrm{~Hz}), 4.79(\mathrm{~s}$, $2 \mathrm{H}), 2.98(\mathrm{dd}, 1 \mathrm{H}, \mathrm{J}=7.1,14.0 \mathrm{~Hz}), 2.77(\mathrm{dd}, 1 \mathrm{H}, \mathrm{J}=6.5,14.0 \mathrm{~Hz}), 1.84(\mathrm{dd}, 3 \mathrm{H}, \mathrm{J}=$ 1.7, $6.9 \mathrm{~Hz}), 1.24(\mathrm{~d}, 3 \mathrm{H}, \mathrm{J}=6.2 \mathrm{~Hz}), 0.93(\mathrm{~s}, 9 \mathrm{H}), 0.10(\mathrm{~s}, 6 \mathrm{H}) .{ }^{13} \mathrm{C}$ NMR $(100 \mathrm{MHz}$; $\left.\mathrm{CDCl}_{3}\right): \delta 166.1,144.5,139.6,135.1,130.4,127.4,127.2,126.8,123.3,71.0,63.2,38.6$, 26.2, 20.0, 18.6, 18.2, -5.1. LRMS (ESI) $\mathrm{m} / \mathrm{z}$ calc'd for $\mathrm{C}_{20} \mathrm{H}_{32} \mathrm{O}_{3} \mathrm{SiNa}[\mathrm{M}+\mathrm{Na}]^{+}: 371.2$; found: 371.2 . 
But-2-enoic acid 2-(2-hydroxymethylphenyl)-1-methylethyl ester (4d):

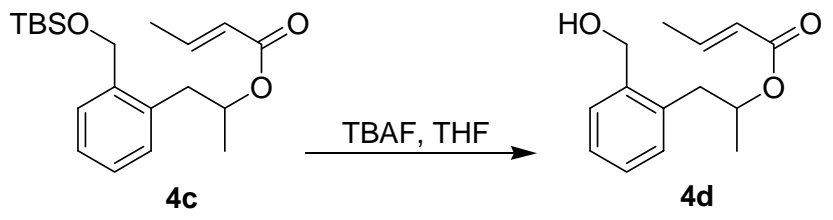

Compound 4d was prepared from 4c by following the procedure for the preparation of 1d and was purified by flash chromatography $(2: 1$ hexanes:EtOAc) to provide a cololess oil $(0.47 \mathrm{~g}, 2.0 \mathrm{mmol}, 65 \%)$.

${ }^{1} \mathrm{H}$ NMR (400 MHz; $\left.\mathrm{CDCl}_{3}\right): \delta$ 7.36-7.31 (m, 1H), 7.23-7.15 (m, 3H), $6.91(\mathrm{dq}$, $1 \mathrm{H}, \mathrm{J}=6.9,15.5 \mathrm{~Hz}), 5.76(\mathrm{dq}, 1 \mathrm{H}, \mathrm{J}=1.7,15.5 \mathrm{~Hz}), 5.17(\mathrm{~h}, 1 \mathrm{H}, \mathrm{J}=6.3 \mathrm{~Hz}), 4.79-4.66$ $(\mathrm{m}, 2 \mathrm{H}), 3.09(\mathrm{dd}, 1 \mathrm{H}, \mathrm{J}=7.0,13.7 \mathrm{~Hz}), 2.81(\mathrm{dd}, 1 \mathrm{H}, \mathrm{J}=6.7,13.7 \mathrm{~Hz}), 2.58(\mathrm{br} \mathrm{s}, 1 \mathrm{H})$, $1.84(\mathrm{dd}, 3 \mathrm{H}, \mathrm{J}=1.7,6.9 \mathrm{~Hz}), 1.29(\mathrm{~d}, 3 \mathrm{H}, \mathrm{J}=6.2 \mathrm{~Hz}) .{ }^{13} \mathrm{C} \mathrm{NMR}\left(100 \mathrm{MHz} ; \mathrm{CDCl}_{3}\right): \delta$ $166.5,145.2$, 139.5, 136.2, 131.1, 129.5, 128.1, 127.3, 122.9, 71.7, 63.6, 39.4, 20.0, 18.2. LRMS (ESI) $m / z$ calc'd for $\mathrm{C}_{14} \mathrm{H}_{18} \mathrm{O}_{3} \mathrm{Na}[\mathrm{M}+\mathrm{Na}]^{+}$: 257.1; found: 257.1 .

\section{But-2-enoic acid 2-(2-formylphenyl)-1-methylethyl ester (4e):}
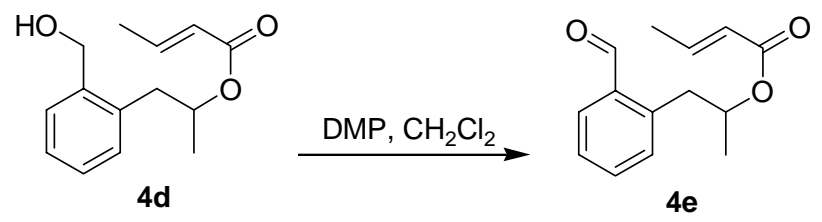

Compound 4e was prepared from 4d by following the procedure for the preparation of $\mathbf{4 a}$ and was purified by flash chromatography (10:1 hexanes:EtOAc) to provide a colorless oil $(0.42 \mathrm{~g}, 1.8 \mathrm{mmol}, 91 \%)$.

${ }^{1} \mathrm{H} \mathrm{NMR}\left(400 \mathrm{MHz} ; \mathrm{CDCl}_{3}\right): \delta 10.27(\mathrm{~s}, 1 \mathrm{H}), 7.82(\mathrm{dd}, 1 \mathrm{H}, \mathrm{J}=1.5,7.7 \mathrm{~Hz}), 7.48$ $(\mathrm{dt}, 1 \mathrm{H}, \mathrm{J}=1.6,7.5 \mathrm{~Hz}), 7.39(\mathrm{dt}, 1 \mathrm{H}, \mathrm{J}=1.3,7.6 \mathrm{~Hz}), 7.27(\mathrm{dd}, 1 \mathrm{H}, \mathrm{J}=0.9,7.6 \mathrm{~Hz})$, $6.86(\mathrm{dq}, 1 \mathrm{H}, \mathrm{J}=6.9,15.6 \mathrm{~Hz}), 5.72(\mathrm{dq}, 1 \mathrm{H}, \mathrm{J}=1.7,15.6 \mathrm{~Hz}), 5.16(\mathrm{ddq}, 1 \mathrm{H}, \mathrm{J}=5.0$, 6.2, $7.8 \mathrm{~Hz}), 3.35(\mathrm{dd}, 1 \mathrm{H}, \mathrm{J}=5.0,13.7 \mathrm{~Hz}), 3.26(\mathrm{dd}, 1 \mathrm{H}, \mathrm{J}=7.9,13.6 \mathrm{~Hz}), 1.82(\mathrm{dd}$, $3 \mathrm{H}, \mathrm{J}=1.7,7.0 \mathrm{~Hz}), 1.30(\mathrm{~d}, 3 \mathrm{H}, \mathrm{J}=6.2 \mathrm{~Hz}) .{ }^{13} \mathrm{C} \mathrm{NMR}\left(100 \mathrm{MHz} ; \mathrm{CDCl}_{3}\right): \delta 192.8$, $166.0,144.8,140.4,134.5,133.7,132.4,132.3,127.4,122.9,71.3,38.5,20.1,18.1$. IR $\left(\mathrm{cm}^{-1}\right): 2980,2935,1716,1699,1656,1293,1267,1187,756$. HRMS (ESI) $\mathrm{m} / \mathrm{z}$ calc'd for $\mathrm{C}_{14} \mathrm{H}_{16} \mathrm{O}_{3} \mathrm{Na}[\mathrm{M}+\mathrm{Na}]^{+}$: 255.0991; found: 255.0994 .

Scheme 4. Preparation of substrate 5d (precursor of lactone 5; Table 2, entry 3)

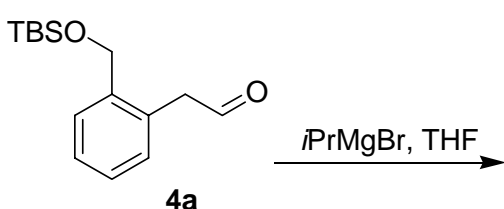

$4 a$
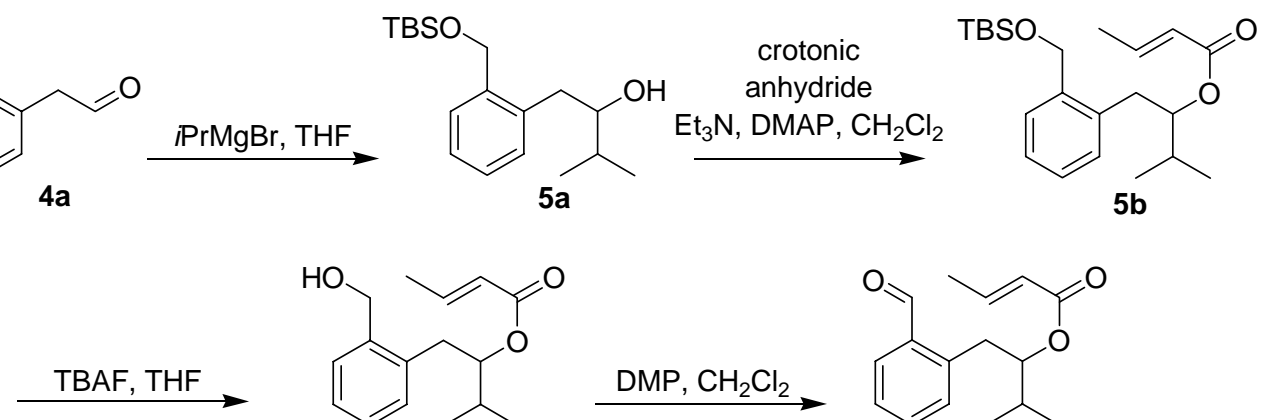<smiles>CC=CC(=O)OC(Cc1ccccc1CO)C(C)C</smiles>
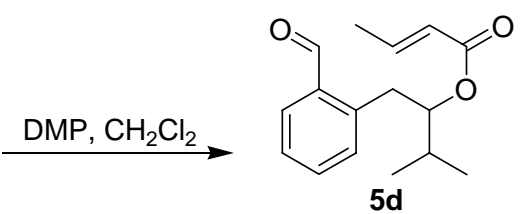
Aldehyde 4a was treated with isopropylmagnesium bromide to obtain alcohol 5a, which was acylated with crotonic anhydride to provide ester $5 \mathbf{b}$. Deprotection of the TBS group in $\mathbf{5 b}$ was accomplished with TBAF, which revealed the alcohol $\mathbf{5 c}$ that was DMP-oxidized to the aldehyde 5d.

1-[2-(tert-Butyldimethylsilanyloxymethyl)phenyl]-3-mehtylbutan-2-ol (5a):

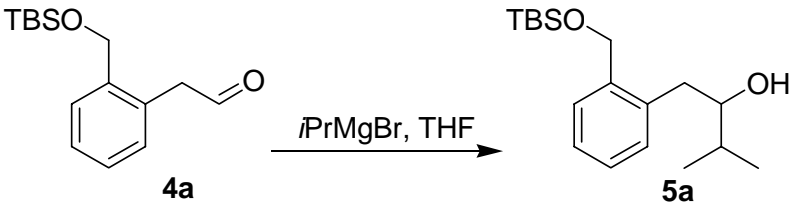

$i \operatorname{PrMgBr}\left(3.05 \mathrm{~mL}, 6.1 \mathrm{mmol}, 2.0 \mathrm{M}\right.$ solution in $\left.\mathrm{Et}_{2} \mathrm{O}\right)$ was added to a stirred solution of aldehyde 4a $(1.08 \mathrm{~g}, 4.1 \mathrm{mmol})$ in THF $(40 \mathrm{~mL})$ at $0^{\circ} \mathrm{C}$. After 1 hour, the reaction was quenched with saturated $\mathrm{NH}_{4} \mathrm{Cl}(20 \mathrm{~mL})$. The organic phase was separated, washed with deionized water and brine, dried over anhydrous $\mathrm{MgSO}_{4}$, and concentrated under reduced pressure on the rotary evaporator. The crude product was purified by flash chromatography (5:1 hexanes:EtOAc) to provide alcohol 5a $(0.94 \mathrm{~g}, 3.0 \mathrm{mmol}, 75 \%)$.

${ }^{1} \mathrm{H}$ NMR $\left(400 \mathrm{MHz} ; \mathrm{CDCl}_{3}\right): \delta$ 7.34-7.30 (m, 1H), 7.27-7.17 (m, 3H), $4.80(\mathrm{~d}$, $1 \mathrm{H}, \mathrm{J}=12.2 \mathrm{~Hz}), 4.63(\mathrm{~d}, 1 \mathrm{H}, \mathrm{J}=12.1 \mathrm{~Hz}), 3.54(\mathrm{ddd}, 1 \mathrm{H}, \mathrm{J}=3.1,4.6,9.5 \mathrm{~Hz}), 2.84$ (dd, $1 \mathrm{H}, \mathrm{J}=2.9,13.8 \mathrm{~Hz}), 2.63(\mathrm{dd}, 1 \mathrm{H}, \mathrm{J}=10.1,13.8 \mathrm{~Hz}), 2.44(\mathrm{br} \mathrm{s}, 1 \mathrm{H}), 1.78(\mathrm{dh}, 1 \mathrm{H}, \mathrm{J}=$ 5.1, $6.9 \mathrm{~Hz}), 1.01(\mathrm{~d}, 3 \mathrm{H}, \mathrm{J}=2.0 \mathrm{~Hz}), 0.99(\mathrm{~d}, 3 \mathrm{H}, \mathrm{J}=2.0 \mathrm{~Hz}), 0.93(\mathrm{~s}, 9 \mathrm{H}), 0.13(\mathrm{~s}, 3 \mathrm{H})$, $0.11(\mathrm{~s}, 3 \mathrm{H}) .{ }^{13} \mathrm{C} \mathrm{NMR}\left(100 \mathrm{MHz} ; \mathrm{CDCl}_{3}\right): \delta 139.0,138.1,130.3,128.7,128.1,126.6$, 76.9, 64.0, 36.8, 34.3, 26.2, 18.8, 18.7, 18.0, -5.0. LRMS (ESI) $\mathrm{m} / \mathrm{z}$ calc'd for $\mathrm{C}_{18} \mathrm{H}_{33} \mathrm{O}_{2} \mathrm{Si}[\mathrm{M}+\mathrm{H}]^{+}$: 309.2; found: 309.3 .

\section{But-2-enoic acid 1-[2-(tert-Butyldimethylsilanyloxymethyl)benzyl]-2-methylpropyl ester (5b):}
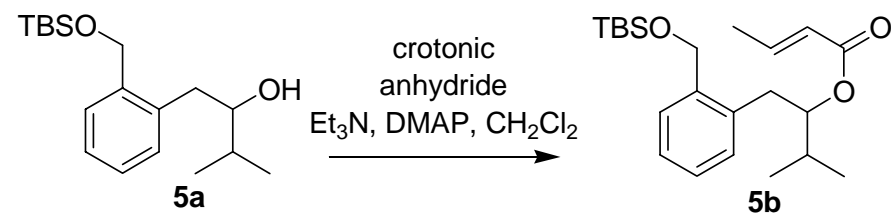

Compound 5b was prepared from $\mathbf{5 a}$ by following the procedure for the preparation of 1c and was purified by flash chromatography (5:1 hexanes:EtOAc) to provide a colorless oil $(0.72 \mathrm{~g}, 1.9 \mathrm{mmol}, 64 \%)$.

${ }^{1} \mathrm{H}$ NMR $\left(400 \mathrm{MHz} ; \mathrm{CDCl}_{3}\right): \delta 7.39-7.36(\mathrm{~m}, 1 \mathrm{H}), 7.20-7.10(\mathrm{~m}, 3 \mathrm{H}), 6.85(\mathrm{dq}$, $1 \mathrm{H}, \mathrm{J}=6.9,15.6 \mathrm{~Hz}), 5.73(\mathrm{dq}, 1 \mathrm{H}, \mathrm{J}=1.7,15.6 \mathrm{~Hz}), 5.01(\mathrm{dt}, 1 \mathrm{H}, \mathrm{J}=4.8,9.4 \mathrm{~Hz}), 4.77$ $(\mathrm{s}, 2 \mathrm{H}), 2.88(\mathrm{dd}, 1 \mathrm{H}, \mathrm{J}=4.9,14.3 \mathrm{~Hz}), 2.81(\mathrm{dd}, 1 \mathrm{H}, \mathrm{J}=8.7,14.3 \mathrm{~Hz}), 1.95-1.84(\mathrm{~m}$, $1 \mathrm{H}), 1.82(\mathrm{dd}, 3 \mathrm{H}, \mathrm{J}=1.7,7.0 \mathrm{~Hz}), 0.98(\mathrm{~d}, 3 \mathrm{H}, \mathrm{J}=6.9 \mathrm{~Hz}), 0.94(\mathrm{~d}, 3 \mathrm{H}, \mathrm{J}=6.9 \mathrm{~Hz})$, $0.92(\mathrm{~s}, 9 \mathrm{H}), 0.08(\mathrm{~s}, 6 \mathrm{H}) .{ }^{13} \mathrm{C} \mathrm{NMR}\left(100 \mathrm{MHz}, \mathrm{CDCl}_{3}\right): \delta 166.2,144.4,139.6,135.5$, 130.2, 127.4, 127.1, 126.7, 123.1, 78.1, 63.2, 33.8, 31.6, 26.2, 19.0, 18.6, 18.2, 17.5, -5.0. LRMS (ESI) $\mathrm{m} / \mathrm{z}$ calc'd for $\mathrm{C}_{22} \mathrm{H}_{36} \mathrm{O}_{3} \mathrm{SiNa}[\mathrm{M}+\mathrm{Na}]^{+}$: 399.2 ; found: 399.4 . 
But-2-enoic acid 1-(2-hydroxymethylbenzyl)-2-methylpropyl ester (5c):

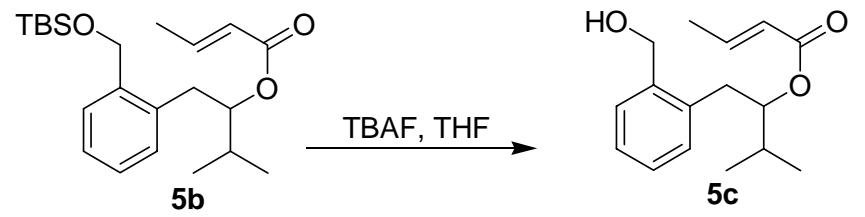

Compound 5c was prepared from $5 \mathbf{b}$ by following the procedure for the preparation of 1d and was purified by flash chromatography $(2: 1$ hexanes:EtOAc) to provide a clear oil ( $0.34 \mathrm{~g}, 1.3 \mathrm{mmol}, 89 \%)$.

${ }^{1} \mathrm{H}$ NMR (400 MHz; $\left.\mathrm{CDCl}_{3}\right): \delta 7.34-7.29(\mathrm{~m}, 1 \mathrm{H}), 7.21-7.11(\mathrm{~m}, 3 \mathrm{H}), 6.83(\mathrm{dq}$, $1 \mathrm{H}, \mathrm{J}=6.9,15.6 \mathrm{~Hz}), 5.70(\mathrm{dq}, 1 \mathrm{H}, \mathrm{J}=1.7,15.6 \mathrm{~Hz}), 5.03(\mathrm{dt}, 1 \mathrm{H}, \mathrm{J}=5.0,9.8 \mathrm{~Hz}), 4.69$ $(\mathrm{d}, 1 \mathrm{H}, \mathrm{J}=1.4 \mathrm{~Hz}), 2.99(\mathrm{dd}, 1 \mathrm{H}, \mathrm{J}=5.2,13.9 \mathrm{~Hz}), 2.87(\mathrm{dd}, 1 \mathrm{H}, \mathrm{J}=9.0,13.9 \mathrm{~Hz}), 2.63$ (br s, $1 \mathrm{H}), 1.98-1.85(\mathrm{~m}, 1 \mathrm{H}), 1.80(\mathrm{dd}, 3 \mathrm{H}, \mathrm{J}=1.7,6.9 \mathrm{~Hz}), 1.01(\mathrm{~d}, 3 \mathrm{H}, \mathrm{J}=6.9 \mathrm{~Hz})$, $0.96(\mathrm{~d}, 3 \mathrm{H}, \mathrm{J}=6.9 \mathrm{~Hz}) .{ }^{13} \mathrm{C}$ NMR $\left(100 \mathrm{MHz} ; \mathrm{CDCl}_{3}\right): \delta 166.5,145.0,139.4,136.3$, 131.0, 129.5, 128.0, 127.1, 122.7, 78.7, 63.4, 34.7, 31.5, 19.1, 18.1, 17.4. LRMS (ESI) $\mathrm{m} / \mathrm{z}$ calc'd for $\mathrm{C}_{16} \mathrm{H}_{22} \mathrm{O}_{3} \mathrm{Na}[\mathrm{M}+\mathrm{Na}]^{+}$: 285.2; found: 285.3 .

But-2-enoic acid 1-(2-formylbenzyl)-2-methylpropyl ester (5d):

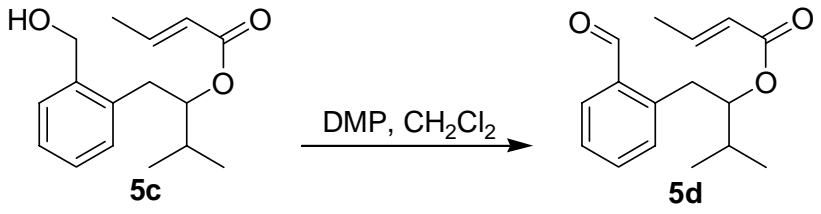

Compound 5d was prepared from 5c by following the procedure for the preparation of $\mathbf{4 a}$ and was purified by flash chromatography $(2: 1$ hexanes:EtOAc) to provide a colorless oil $(0.29 \mathrm{~g}, 1.1 \mathrm{mmol}, 97 \%)$.

${ }^{1} \mathrm{H}$ NMR (400 MHz; $\left.\mathrm{CDCl}_{3}\right): \delta 10.23(\mathrm{~s}, 1 \mathrm{H}), 7.77(\mathrm{dd}, 1 \mathrm{H}, \mathrm{J}=1.5,7.6 \mathrm{~Hz}), 7.44$ $(\mathrm{dt}, 1 \mathrm{H}, \mathrm{J}=1.5,7.4 \mathrm{~Hz}), 7.35(\mathrm{dt}, 1 \mathrm{H}, \mathrm{J}=1.3,7.6 \mathrm{~Hz}), 7.25-7.21(\mathrm{~m}, 1 \mathrm{H}), 6.78(\mathrm{dq}, 1 \mathrm{H}, \mathrm{J}$ $=7.0,15.6 \mathrm{~Hz}), 5.66(\mathrm{dq}, 1 \mathrm{H}, \mathrm{J}=1.7,15.6 \mathrm{~Hz}), 5.00(\mathrm{ddd}, 1 \mathrm{H}, \mathrm{J}=3.5,5.1,10.0 \mathrm{~Hz})$, $3.44(\mathrm{dd}, 1 \mathrm{H}, \mathrm{J}=3.4,13.6 \mathrm{~Hz}), 3.09(\mathrm{dd}, 1 \mathrm{H}, \mathrm{J}=10.0,13.6 \mathrm{~Hz}), 2.00-1.88(\mathrm{~m}, 1 \mathrm{H}), 1.79$ $(\mathrm{dd}, 3 \mathrm{H}, \mathrm{J}=1.7,6.9 \mathrm{~Hz}), 1.01(\mathrm{~d}, 3 \mathrm{H}, \mathrm{J}=3.2 \mathrm{~Hz}), 0.98(\mathrm{~d}, 3 \mathrm{H}, \mathrm{J}=3.2 \mathrm{~Hz}) .{ }^{13} \mathrm{C} \mathrm{NMR}$ $\left(100 \mathrm{MHz} ; \mathrm{CDCl}_{3}\right): \delta 192.9,165.9,144.6,141.0,134.5,133.6,132.6,132.2,127.2$, 122.7, 78.4, 34.3, 32.4, 18.8, 18.1, 17.8. IR ( $\left.\mathrm{cm}^{-1}\right): 2967,2876,2735,1716,1698,1659$, 1292, 1263, 1183, 1009, 970, 757. HRMS (ESI) $\mathrm{m} / \mathrm{z}$ calc'd for $\mathrm{C}_{16} \mathrm{H}_{21} \mathrm{O}_{3}[\mathrm{M}+\mathrm{H}]^{+}$: 261.1487; found: 261.1485 . 
Scheme5. Preparation of substrate 6e (precursor of lactone 6; Table 2, entry 4)
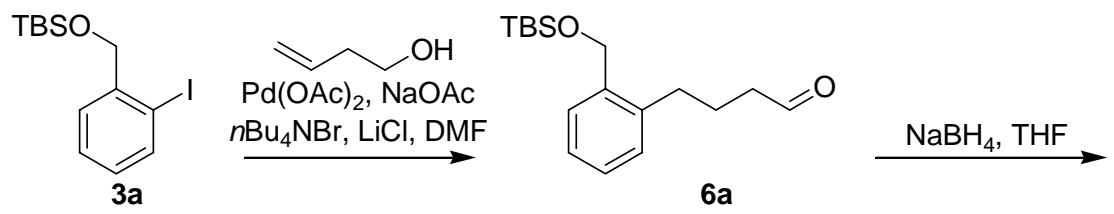

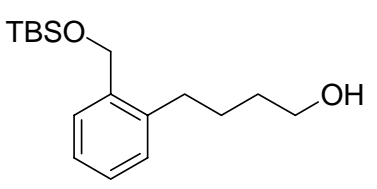

$6 b$

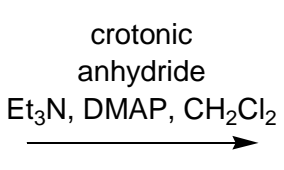

anhydride<smiles>C/C=C\C(=O)OCCCCc1ccccc1CO</smiles>

6d

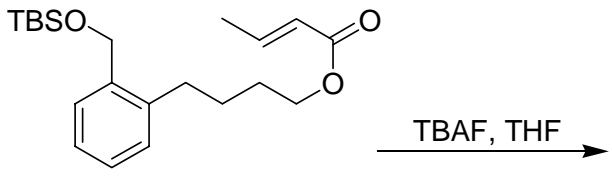

$6 c$ $\stackrel{\text { DMP }, \mathrm{CH}_{2} \mathrm{Cl}_{2}}{\longrightarrow}$

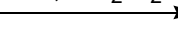

A coupling reaction ${ }^{5}$ between TBS ether $3 \mathbf{a}^{3}$ and 3-buten-1-ol provided aldehyde 6a, which was reduced with sodium borohydride to afford alcohol $\mathbf{6 b}$. Acylation with crotonic anhydride followed by a TBS deprotection resulted in alcohol 6d, which was oxidized with DMP to aldehyde $6 \mathbf{e}$.

\section{4-[2-(tert-Butyldimethylsilanyloxymethyl)phenyl]butyraldehyde (6a):}
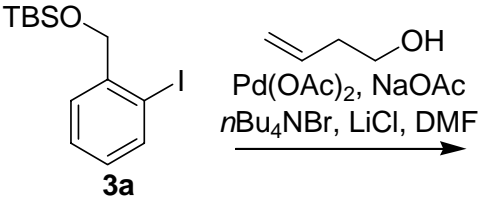<smiles>CC(C)COc1ccccc1CCCC=O</smiles>

$6 a$

$n \mathrm{Bu}_{4} \mathrm{NBr}(19.35 \mathrm{~g}, 60 \mathrm{mmol}), \mathrm{NaOAc}(6.15 \mathrm{~g}, 75 \mathrm{mmol}), \mathrm{LiCl}(1.28 \mathrm{~g}, 30 \mathrm{mmol})$, and $\mathrm{Pd}(\mathrm{OAc})_{2}(0.6 \mathrm{~g}, 2.6 \mathrm{mmol})$ were added sequentially to a stirred solution of $3 \mathrm{a}^{3}(10.0$ $\mathrm{g}, 28.7 \mathrm{mmol})$ and 3-buten-1-ol $(2.61 \mathrm{~mL}, 30 \mathrm{mmol})$ in anhydrous DMF $(60 \mathrm{~mL}){ }^{5}$ The mixture was heated to $50^{\circ} \mathrm{C}$. After 3 days at $50^{\circ} \mathrm{C}$, the reaction mixture was cooled to room temperature and diluted with EtOAc $(100 \mathrm{~mL})$ and hexanes $(200 \mathrm{~mL})$ resulting in a biphasic organic solution. The DMF-containing phase was separated and extracted with hexanes $(100 \mathrm{~mL})$. The combined hexanes/EtOAc extracts were washed with deionized water, 1.0 $\mathrm{M} \mathrm{CuSO}_{4}$, deionized water, $1.0 \mathrm{M} \mathrm{NaOH}$, deionized water, and brine. After drying over anhydrous $\mathrm{MgSO}_{4}$ and concentrating under reduced pressure, the crude product was purified by flash chromatography (30:1 hexanes:EtOAc) to afford aldehyde 6a $(4.4 \mathrm{~g}, 15.1 \mathrm{mmol}, 53 \%)$.

${ }^{1} \mathrm{H}$ NMR $\left(500 \mathrm{MHz} ; \mathrm{CDCl}_{3}\right): \delta 9.76(\mathrm{t}, 1 \mathrm{H}, \mathrm{J}=1.5 \mathrm{~Hz}), 7.42-7.38(\mathrm{~m}, 1 \mathrm{H}), 7.22-$ $7.17(\mathrm{~m}, 2 \mathrm{H}), 7.14-7.10(\mathrm{~m}, 1 \mathrm{H}), 4.72(\mathrm{~s}, 2 \mathrm{H}), 2.64(\mathrm{t}, 2 \mathrm{H}, \mathrm{J}=7.7 \mathrm{~Hz}), 2.48(\mathrm{dt}, 2 \mathrm{H}, \mathrm{J}=$ $1.5,7.3 \mathrm{~Hz}), 1.96-1.89(\mathrm{~m}, 2 \mathrm{H}), 0.92(\mathrm{~m}, 2 \mathrm{H}), 0.09(\mathrm{~s}, 6 \mathrm{H}) .{ }^{13} \mathrm{C}$ NMR $(100 \mathrm{MHz}$; $\left.\mathrm{CDCl}_{3}\right): \delta 202.5,139.0,138.7,129.2,127.7,127.5,126.5,63.2,43.7,31.5,26.2,23.2$, 18.7, -5.0. LRMS (ESI) $\mathrm{m} / \mathrm{z}$ calc'd for $\mathrm{C}_{17} \mathrm{H}_{28} \mathrm{O}_{2} \mathrm{SiNa}[\mathrm{M}+\mathrm{Na}]^{+}$: 315.2; found: 315.1 .

\footnotetext{
${ }^{5}$ Procedure adapted from: Larock, R. C.; Leung, W-Y.; Stolz-Dunn, S. Tetrahedron Lett. 1989, 30, 6629.
} 


\section{4-[2-(tert-Butyldimethylsilanyloxymethyl)phenyl]butan-1-ol (6b):}

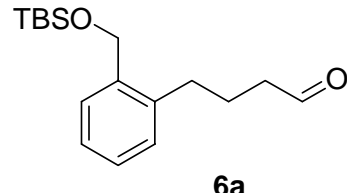

$6 a$

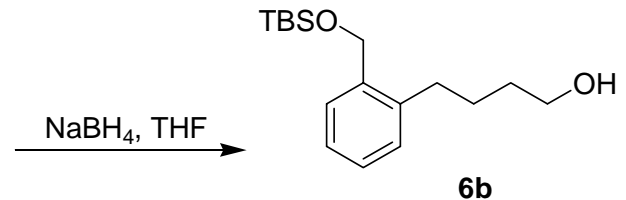

$6 b$

$\mathrm{NaBH}_{4}(0.15 \mathrm{~g}, 4.0 \mathrm{mmol})$ was added to a stirred solution of 6a $(1.06 \mathrm{~g}, 3.6$ $\mathrm{mmol})$ in THF $(30 \mathrm{~mL})$. After 1 hour, the reaction was slowly quenched with $\mathrm{HCl}(10$ $\mathrm{mL}, 1 \mathrm{M}$ solution in water). After diluting with $\mathrm{Et}_{2} \mathrm{O}(30 \mathrm{~mL})$, the organic phase was separated, washed with deionized water and brine, dried over anhydrous $\mathrm{MgSO}_{4}$, and concentrated under reduced pressure on the rotary evaporator. $\mathrm{MeOH}(30 \mathrm{~mL})$ was added and then removed under reduced pressure on the rotary evaporator. After repeating the $\mathrm{MeOH}$ exchange once more, the crude product was purified by flash chromatography (5:1 hexanes:EtOAc) to provide alcohol $\mathbf{6 b}(0.96 \mathrm{~g}, 3.1 \mathrm{mmol}, 86 \%)$.

${ }^{1} \mathrm{H}$ NMR (500 MHz; $\left.\mathrm{CDCl}_{3}\right): \delta 7.42-7.37(\mathrm{~m}, 1 \mathrm{H}), 7.21-7.11(\mathrm{~m}, 3 \mathrm{H}), 4.73(\mathrm{~s}$, 2H), 3.65 (t, 2H, J = 6.3 Hz), $2.62(\mathrm{t}, 2 \mathrm{H}, \mathrm{J}=7.5 \mathrm{~Hz}), 1.70-1.59(\mathrm{~m}, 4 \mathrm{H}), 1.34(\mathrm{br} \mathrm{s}, 1 \mathrm{H})$, $0.92(\mathrm{~s}, 9 \mathrm{H}), 0.08(\mathrm{~s}, 6 \mathrm{H}) .{ }^{13} \mathrm{C} \mathrm{NMR}\left(100 \mathrm{MHz} ; \mathrm{CDCl}_{3}\right): \delta 139.7,138.8,129.1,127.5$, 127.4, 126.2, 63.3, 63.1, 32.9, 32.0, 27.0, 26.2, 18.7, -5.0. LRMS (ESI) $\mathrm{m} / \mathrm{z}$ calc'd for $\mathrm{C}_{17} \mathrm{H}_{30} \mathrm{O}_{2} \mathrm{SiNa}[\mathrm{M}+\mathrm{Na}]^{+}$: 317.2 ; found: 317.1 .

\section{But-2-enoic acid 4-[2-(tert-Butyldimethylsilanyloxymethyl)phenyl]butyl ester (6c):}
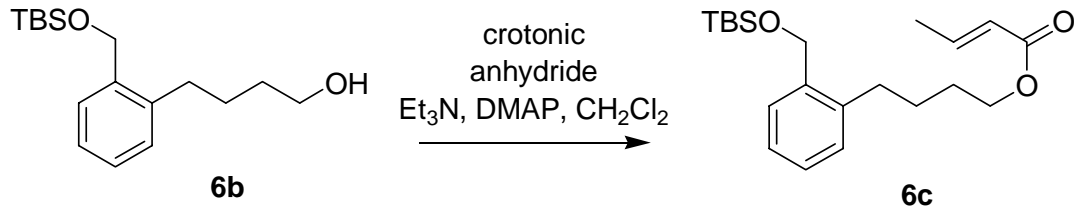

Compound 6c was prepared from $\mathbf{6 b}$ by following the procedure for the preparation of 1c and was purified by flash chromatography (15:1 hexanes:EtOAc) to provide a colorless oil $(0.68 \mathrm{~g}, 70 \%)$.

${ }^{1} \mathrm{H}$ NMR $\left(400 \mathrm{MHz} ; \mathrm{CDCl}_{3}\right): \delta 7.42-7.37(\mathrm{~m}, 1 \mathrm{H}), 7.21-7.10(\mathrm{~m}, 3 \mathrm{H}), 6.95(\mathrm{dq}$, $1 \mathrm{H}, \mathrm{J}=7.0,15.6 \mathrm{~Hz}), 5.82(\mathrm{dq}, 1 \mathrm{H}, \mathrm{J}=1.6,15.5 \mathrm{~Hz}), 4.72(\mathrm{~s}, 2 \mathrm{H}), 4.13(\mathrm{t}, 2 \mathrm{H}, \mathrm{J}=6.2$ $\mathrm{Hz}), 2.62(\mathrm{t}, 2 \mathrm{H}, \mathrm{J}=8.0 \mathrm{~Hz}), 1.85(\mathrm{dd}, 3 \mathrm{H}, \mathrm{J}=1.6,7.0 \mathrm{~Hz}), 1.76-1.60(\mathrm{~m}, 4 \mathrm{H}), 0.92(\mathrm{~s}$, 9H), $0.08(\mathrm{~s}, 6 \mathrm{H}) .{ }^{13} \mathrm{C}$ NMR $\left(100 \mathrm{MHz} ; \mathrm{CDCl}_{3}\right): \delta 166.8,144.8,139.4,138.9,129.0$, 127.4, 127.3, 126.2, 123.0, 64.3, 63.1, 53.6, 31.9, 29.0, 27.3, 26.2, 18.7, 18.2, -5.0. LRMS (ESI) $\mathrm{m} / \mathrm{z}$ calc'd for $\mathrm{C}_{21} \mathrm{H}_{34} \mathrm{O}_{3} \mathrm{SiNa}[\mathrm{M}+\mathrm{Na}]^{+}$: 385.2; found: 385.2 .

\section{But-2-enoic acid 4-(2-hydroxymethylphenyl)butyl ester (6d):}
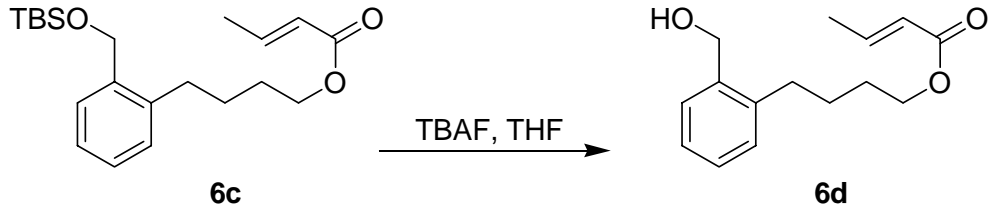

Compound 6d was prepared from 6c by following the procedure for the preparation of 1d and was purified by flash chromatography (5:1 hexanes:EtOAc) to provide a colorless oil $(0.43 \mathrm{~g}, 99 \%)$. 
NMR (400 MHz; $\left.\mathrm{CDCl}_{3}\right): \delta$ 7.36-7.33 (m, 1H), 7.25-7.15 (m, 3H), $6.94(\mathrm{dq}, 1 \mathrm{H}$, $\mathrm{J}=7.0,15.6 \mathrm{~Hz}), 5.81(\mathrm{dq}, 1 \mathrm{H}, \mathrm{J}=1.7,15.5 \mathrm{~Hz}), 4.68(\mathrm{~d}, 2 \mathrm{H}, \mathrm{J}=5.5 \mathrm{~Hz}), 4.13(\mathrm{t}, 2 \mathrm{H}, \mathrm{J}$ $=6.2 \mathrm{~Hz}), 2.69(\mathrm{t}, 2 \mathrm{H}, \mathrm{J}=8.0 \mathrm{~Hz}), 1.89(\mathrm{t}, 1 \mathrm{H}, \mathrm{J}=5.7 \mathrm{~Hz}), 1.85(\mathrm{dd}, 3 \mathrm{H}, \mathrm{J}=1.7,7.0 \mathrm{~Hz})$, 1.76-1.61 (m, 4H). ${ }^{13} \mathrm{C}$ NMR $\left(100 \mathrm{MHz} ; \mathrm{CDCl}_{3}\right): \delta 166.9,144.9,140.4,138.5,129.6$, $128.5,128.2,126.5,122.8,64.2,63.2,32.0,28.8,27.7,18.2$. LRMS (ESI) $\mathrm{m} / \mathrm{z}$ calc'd for $\mathrm{C}_{15} \mathrm{H}_{20} \mathrm{O}_{3} \mathrm{Na}[\mathrm{M}+\mathrm{Na}]^{+}:$271.1; found: 271.1.

But-2-enoic acid 4-(2-formylphenyl)butyl ester (6e):

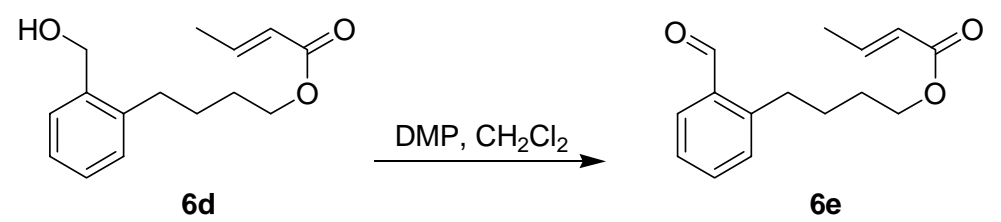

Compound 6e was prepared from $\mathbf{6 d}$ by following the procedure for the preparation of $\mathbf{4 a}$ and was purified by flash chromatography (10:1 hexanes:EtOAc) to provide a colorless oil $(0.32 \mathrm{~g}, 89 \%)$.

${ }^{1} \mathrm{H}$ NMR $\left(400 \mathrm{MHz} ; \mathrm{CDCl}_{3}\right): \delta 10.23(\mathrm{~s}, 1 \mathrm{H}), 7.80(\mathrm{dd}, 1 \mathrm{H}, \mathrm{J}=1.5,7.6 \mathrm{~Hz}), 7.48$ $(\mathrm{dt}, 1 \mathrm{H}, \mathrm{J}=1.5,7.5 \mathrm{~Hz}), 7.35(\mathrm{dt}, 1 \mathrm{H}, \mathrm{J}=4.2,7.5 \mathrm{~Hz}), 7.25(\mathrm{~d}, 1 \mathrm{H}, \mathrm{J}=8.8 \mathrm{~Hz}), 6.94(\mathrm{dq}$, $1 \mathrm{H}, \mathrm{J}=6.9,15.5 \mathrm{~Hz}), 5.82(\mathrm{dq}, 1 \mathrm{H}, \mathrm{J}=1.7,15.6 \mathrm{~Hz}), 4.13(\mathrm{t}, 2 \mathrm{H}, \mathrm{J}=6.2 \mathrm{~Hz}), 3.05(\mathrm{t}$, $2 \mathrm{H}, \mathrm{J}=7.8 \mathrm{~Hz}), 1.85(\mathrm{dd}, 3 \mathrm{H}, \mathrm{J}=1.7,6.9 \mathrm{~Hz}), 1.78-1.62(\mathrm{~m}, 4 \mathrm{H}) .{ }^{13} \mathrm{C} \mathrm{NMR}(100 \mathrm{MHz}$; $\left.\mathrm{CDCl}_{3}\right): \quad \delta 192.7,166.8,145.1,144.9,134.0,133.9,132.6,131.3,126.9,122.9,64.1$, 32.4, 28.8, 28.7, 18.2. IR $\left(\mathrm{cm}^{-1}\right): 2947,2735,1718,1698,1659,1312,1267,1185,757$. HRMS (ESI) $\mathrm{m} / \mathrm{z}$ calc'd for $\mathrm{C}_{15} \mathrm{H}_{18} \mathrm{O}_{3} \mathrm{Na}$ [M+Na] $]^{+}: 269.1148$; found: 269.1158 .

Scheme 6. Preparation of substrate 7d (precursor of lactone 7; Table 2, entry 5)

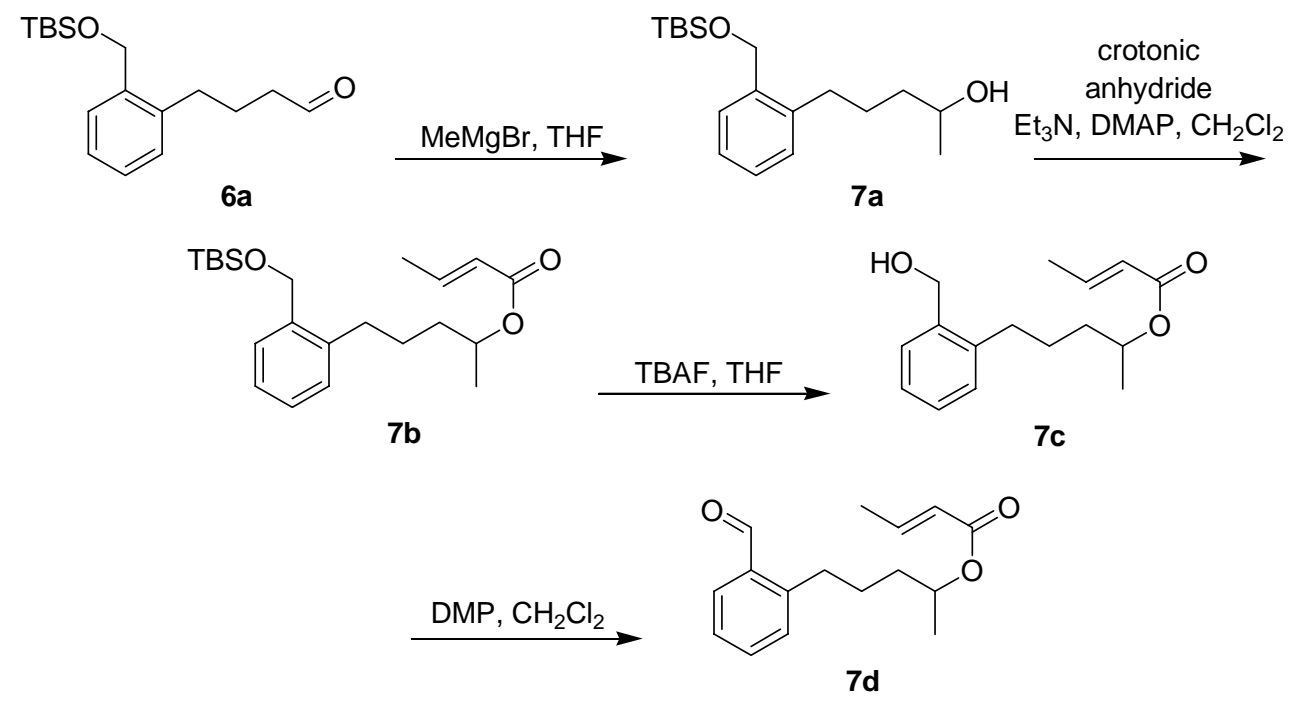

The addition of methylmagnesium bromide to aldehyde $\mathbf{6 a}$ provided alcohol $\mathbf{7 a}$, which was acylated with crotonic anhydride to afford ester $\mathbf{7 b}$. Treatment with TBAF resulted in alcohol 7c, which was oxidized to the aldehyde $\mathbf{7 d}$ with DMP. 


\section{5-[2-(tert-Butyldimethylsilanyloxymethyl)phenyl]pentan-2-ol (7a):}

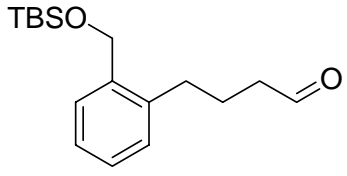

$6 a$

\section{MeMgBr, THF}

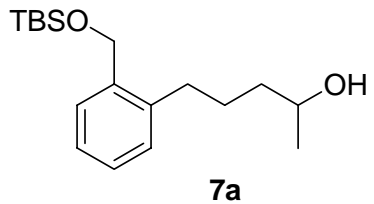

Compound 7a was prepared from 6a according to the procedure for the preparation of $\mathbf{4 b}$ and was purified by flash chromatography (10:1 hexanes:EtOAc) to provide a colorless oil $(1.90 \mathrm{~g}, 83 \%)$.

${ }^{1} \mathrm{H}$ NMR $\left(400 \mathrm{MHz} ; \mathrm{CDCl}_{3}\right): \delta$ 7.45-7.40 (m, 1H), 7.23-7.14 (m, 3H), $4.76(\mathrm{~s}$, 2H), $3.81(\mathrm{~h}, 1 \mathrm{H}, \mathrm{J}=6.2 \mathrm{~Hz}), 2.69-2.57(\mathrm{~m}, 2 \mathrm{H}), 1.79-1.44(\mathrm{~m}, 5 \mathrm{H}), 1.18(\mathrm{~d}, 3 \mathrm{H}, \mathrm{J}=6.2)$, $0.96(\mathrm{~s}, 9 \mathrm{H}), 0.11(\mathrm{~s}, 6 \mathrm{H}) .{ }^{13} \mathrm{C} \mathrm{NMR}\left(100 \mathrm{MHz}, \mathrm{CDCl}_{3}\right): \quad \delta$ 139.7, 138.8, 129.0, 127.3, 127.2, 126.1, 68.1, 63.2, 39.4, 32.1, 27.0, 26.2, 23.7, 18.6, -5.0. LRMS (ESI) m/z calc'd for $\mathrm{C}_{18} \mathrm{H}_{32} \mathrm{O}_{2} \mathrm{SiNa}[\mathrm{M}+\mathrm{Na}]^{+}$: 331.2; found: 331.2 .

\section{But-2-enoic acid 4-[2-(tert-Butyldimethylsilanyloxymethyl)phenyl]-1-methylbutyl ester (7b):}
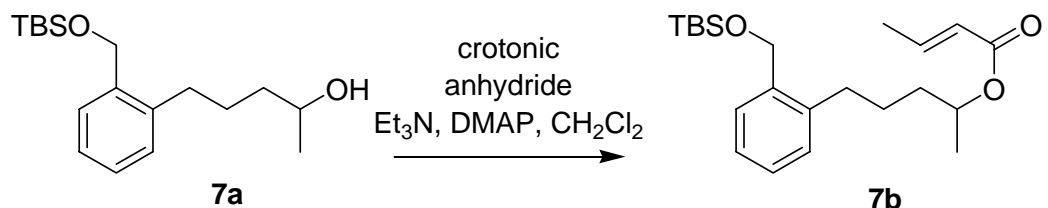

Compound 7b was prepared from 7a by following the procedure for the preparation of 1c and was purified by flash chromatography (30:1 hexanes:EtOAc) to provide a colorless oil $(1.25 \mathrm{~g}, 80 \%)$.

${ }^{1} \mathrm{H}$ NMR $\left(400 \mathrm{MHz} ; \mathrm{CDCl}_{3}\right): \delta$ 7.43-7.38 (m, 1H), 7.20-7.09 $(\mathrm{m}, 3 \mathrm{H}), 6.93(\mathrm{dq}$, $1 \mathrm{H}, \mathrm{J}=7.0,15.6 \mathrm{~Hz}), 5.80(\mathrm{dq}, 1 \mathrm{H}, \mathrm{J}=1.7,15.5 \mathrm{~Hz}), 5.03-4.94(\mathrm{~m}, 1 \mathrm{H}), 4.71(\mathrm{~s}, 2 \mathrm{H})$, 2.64-2.52 (m, 2H), $1.85(\mathrm{dd}, 3 \mathrm{H}, \mathrm{J}=1.7,7.0 \mathrm{~Hz}), 1.72-1.52(\mathrm{~m}, 4 \mathrm{H}), 1.22(\mathrm{~d}, 3 \mathrm{H}, \mathrm{J}=6.2$ $\mathrm{Hz}), 0.92(\mathrm{~s}, 9 \mathrm{H}), 0.08(\mathrm{~s}, 6 \mathrm{H}) .{ }^{13} \mathrm{C} \mathrm{NMR}\left(100 \mathrm{MHz} ; \mathrm{CDCl}_{3}\right): \delta 166.5,144.5,139.4$, 138.9, 129.0, 127.3, 127.2, 126.2, 123.4, 70.7, 63.1, 36.2, 32.1, 26.7, 26.2, 20.3, 18.7, 18.2, -5.0. LRMS (ESI) $\mathrm{m} / \mathrm{z}$ calc'd for $\mathrm{C}_{22} \mathrm{H}_{36} \mathrm{O}_{3} \mathrm{SiNa}[\mathrm{M}+\mathrm{Na}]^{+}$: 399.2; found: 399.2 .

\section{But-2-enoic acid 4-(2-hydroxymethylphenyl)-1-methylbutyl ester (7c):}

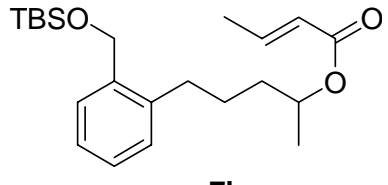

$7 \mathbf{b}$

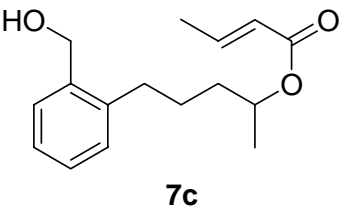

7c

Compound 7c was prepared from $\mathbf{7 b}$ by following the procedure for the preparation of 1d and was purified by flash chromatography (10:1 hexanes:EtOAc) to provide a colorless oil $(0.80 \mathrm{~g}, 95 \%)$.

${ }^{1} \mathrm{H}$ NMR $\left(500 \mathrm{MHz} ; \mathrm{CDCl}_{3}\right): \delta 7.36-7.33(\mathrm{~m}, 1 \mathrm{H}), 7.25-7.14(\mathrm{~m}, 3 \mathrm{H}), 6.93(\mathrm{dq}$, $1 \mathrm{H}, \mathrm{J}=6.8,15.4 \mathrm{~Hz}), 5.80(\mathrm{dq}, 1 \mathrm{H}, \mathrm{J}=1.5,15.5 \mathrm{~Hz}), 5.03-4.95(\mathrm{~m}, 1 \mathrm{H}), 4.68(\mathrm{~s}, 2 \mathrm{H})$, 2.74-2.60 (m, 2H), $1.84(\mathrm{dd}, 3 \mathrm{H}, \mathrm{J}=1.5,6.8 \mathrm{~Hz}), 1.76-1.52(\mathrm{~m}, 5 \mathrm{H}), 1.21(\mathrm{~d}, 3 \mathrm{H}, \mathrm{J}=6.3$ $\mathrm{Hz}) .{ }^{13} \mathrm{C} \mathrm{NMR}\left(100 \mathrm{MHz} ; \mathrm{CDCl}_{3}\right): \delta 166.6,144.6,140.6,138.5,129.6,128.6,128.2$, 
$126.5,123.3,70.5,63.3,36.2,32.2,27.2,20.4,18.2$. LRMS (ESI) $\mathrm{m} / \mathrm{z}$ calc'd for $\mathrm{C}_{16} \mathrm{H}_{22} \mathrm{O}_{3} \mathrm{Na}[\mathrm{M}+\mathrm{Na}]^{+}: 385.1$; found: 285.1 .

But-2-enoic acid 4-(2-formylphenyl)-1-methylbutyl ester (7d):
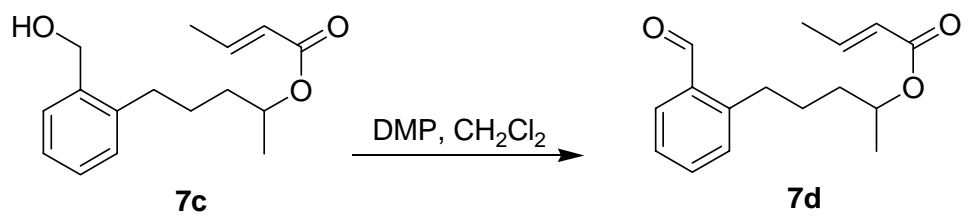

Compound 7d was prepared from 7c by following the procedure for the preparation of 4a and was purified by flash chromatography (15:1 hexanes:EtOAc) to provide a colorless oil $(0.67 \mathrm{~g}, 88 \%)$.

${ }^{1} \mathrm{H}$ NMR $\left(400 \mathrm{MHz} ; \mathrm{CDCl}_{3}\right): \delta 10.23(\mathrm{~s}, 1 \mathrm{H}), 7.80(\mathrm{dd}, 1 \mathrm{H}, \mathrm{J}=1.4,7.6 \mathrm{~Hz}), 7.48$ (dt, $1 \mathrm{H}, \mathrm{J}=1.5,6.0 \mathrm{~Hz}), 7.35(\mathrm{dt}, 1 \mathrm{H}, \mathrm{J}=1.1,7.5 \mathrm{~Hz}), 7.24(\mathrm{br} \mathrm{d}, 1 \mathrm{H}, \mathrm{J}=7.6 \mathrm{~Hz}), 6.92$ $(\mathrm{dq}, 1 \mathrm{H}, \mathrm{J}=6.9,15.5 \mathrm{~Hz}), 5.79(\mathrm{dq}, 1 \mathrm{H}, \mathrm{J}=1.7,15.5 \mathrm{~Hz}), 5.02-4.94(\mathrm{~m}, 1 \mathrm{H}), 3.08-2.95$ $(\mathrm{m}, 2 \mathrm{H}), 1.84(\mathrm{dd}, 3 \mathrm{H}, \mathrm{J}=1.7,6.9 \mathrm{~Hz}), 1.74-1.54(\mathrm{~m}, 4 \mathrm{H}), 1.21(\mathrm{~d}, 3 \mathrm{H}, \mathrm{J}=6.3 \mathrm{~Hz}) .{ }^{13} \mathrm{C}$ NMR $\left(100 \mathrm{MHz} ; \mathrm{CDCl}_{3}\right): \delta 192.6,166.5,145.3,144.6,134.0,133.9,132.4,131.3$, 126.8, 123.4, 70.5, 36.0, 32.6, 28.2, 20.3, 18.2. IR $\left(\mathrm{cm}^{-1}\right): 2935,2849,1714,1698,1658$, 1292, 1189, 756. HRMS (ESI) $\mathrm{m} / \mathrm{z}$ calc'd for $\mathrm{C}_{16} \mathrm{H}_{20} \mathrm{O}_{3} \mathrm{~K}[\mathrm{M}+\mathrm{K}]^{+}$: 299.1044; found: 299.1053.

Scheme 7. Preparation of substrate 8d (precursor of lactone 8; Table 2, entry 6)

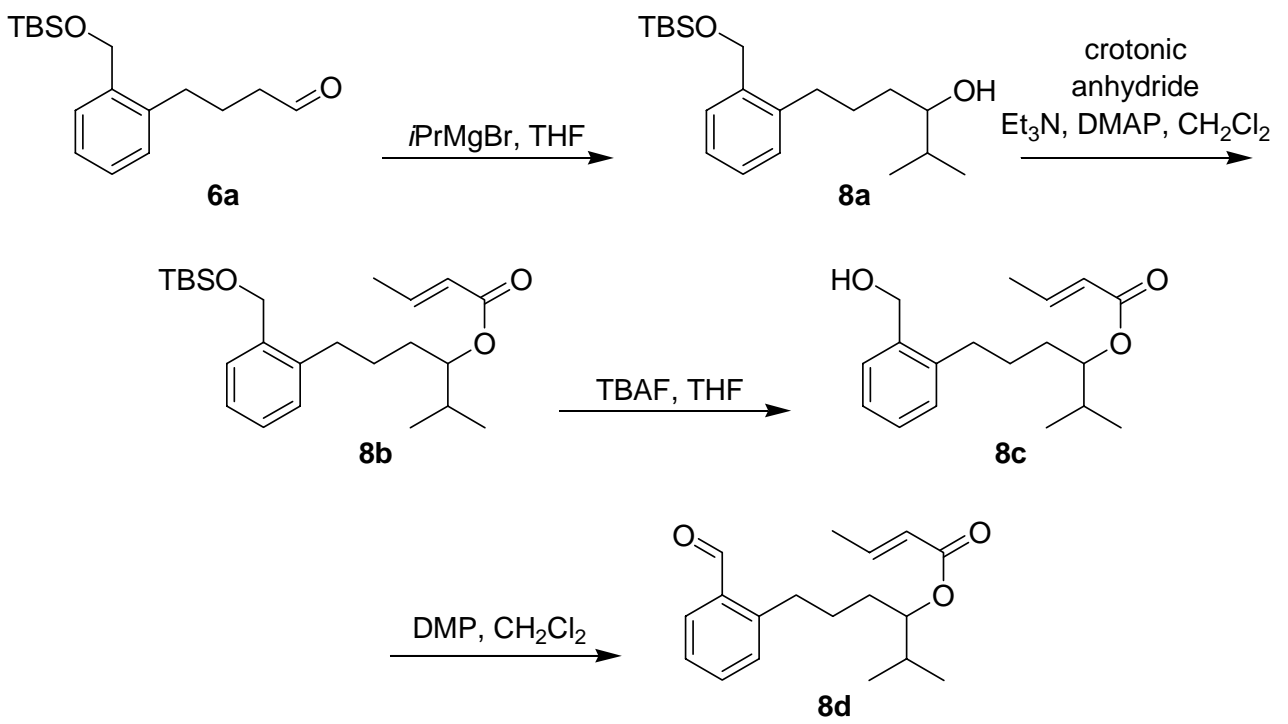

Treatment of aldehyde 6a with isopropylmagnesium bromide resulted in alcohol $\mathbf{8 a}$, which was acylated with crotonic anhydride to afford ester $\mathbf{8 b}$. The deprotection of the TBS group was achieved with TBAF to provide alcohol 8c, which was oxidized to aldehyde 8d with DMP. 


\section{6-[2-(tert-Butyldimethylsilanyloxymethyl)phenyl]-2-methylhexan-3-ol (8a):}

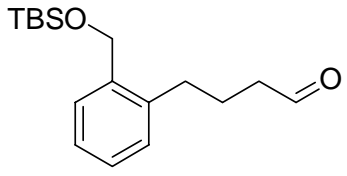

$6 a$ $\stackrel{i \mathrm{PrMgBr}, \mathrm{THF}}{\longrightarrow}$<smiles>CC(C)C(O)CCCc1ccccc1COS(C)(=O)=O</smiles>

Compound 8a was prepared from $\mathbf{6 a}$ according to the procedure for the preparation of $\mathbf{5 a}$ and was purified by flash chromatography (10:1 hexanes:EtOAc) to provide a colorless oil $(0.78 \mathrm{~g}, 78 \%)$.

${ }^{1} \mathrm{H}$ NMR $\left(400 \mathrm{MHz} ; \mathrm{CDCl}_{3}\right): \delta$ 7.42-7.38 (m, $\left.1 \mathrm{H}\right), 7.21-7.12(\mathrm{~m}, 3 \mathrm{H}), 4.74(\mathrm{~s}$, $2 \mathrm{H}), 3.42-3.34(\mathrm{~m}, 1 \mathrm{H}), 2.68-2.54(\mathrm{~m}, 2 \mathrm{H}), 1.85-1.40(\mathrm{~m}, 5 \mathrm{H}), 1.31(\mathrm{~d}, 1 \mathrm{H}, \mathrm{J}=5.0 \mathrm{~Hz})$, $0.93(\mathrm{~s}, 9 \mathrm{H}), 0.91(\mathrm{~d}, 3 \mathrm{H}, \mathrm{J}=4.1 \mathrm{~Hz}), 0.88(\mathrm{~d}, 3 \mathrm{H}, \mathrm{J}=4.0 \mathrm{~Hz}), 0.09(\mathrm{~s}, 6 \mathrm{H}) .{ }^{13} \mathrm{C} \mathrm{NMR}$ $\left(100 \mathrm{MHz} ; \mathrm{CDCl}_{3}\right): \delta$ 139.7, 138.8, 129.0, 127.3, 126.1, 76.8, 63.2, 34.4, 33.7, 32.3, 27.3, 26.2, 19.1, 18.6, 17.3, -5.0. LRMS (ESI) $\mathrm{m} / \mathrm{z}$ calc'd for $\mathrm{C}_{20} \mathrm{H}_{36} \mathrm{O}_{2} \mathrm{SiNa}[\mathrm{M}+\mathrm{Na}]^{+}$: 359.2; found: 359.2 .

\section{But-2-enoic acid 4-[2-(tert-Butyldimethylsilanyloxymethyl)phenyl]-1-isopropylbutyl ester (8b):}

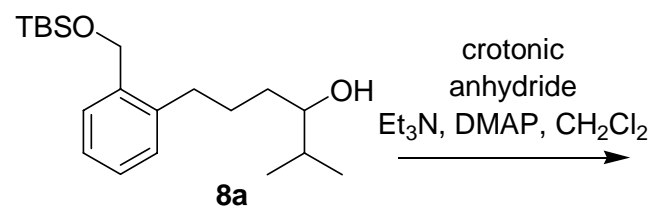<smiles>CC=CC(=O)OC(CCCc1ccccc1CO[AsH3])C(C)C</smiles>

Compound $\mathbf{8 b}$ was prepared from 8a by following the procedure for the preparation of 1c and was purified by flash chromatography (30:1 hexanes:EtOAc) to provide a colorless oil $(0.80 \mathrm{~g}, 80 \%)$.

${ }^{1} \mathrm{H}$ NMR (500 MHz; $\left.\mathrm{CDCl}_{3}\right): \delta$ 7.42-7.38 (m, 1H), 7.19-7.14 (m, 2H), 7.12-7.08 $(\mathrm{m}, 1 \mathrm{H}), 6.94(\mathrm{dq}, 1 \mathrm{H}, \mathrm{J}=6.9,15.5 \mathrm{~Hz}), 5.83(\mathrm{dq}, 1 \mathrm{H}, \mathrm{J}=1.7,15.5 \mathrm{~Hz}), 4.86-4.81(\mathrm{~m}$, $1 \mathrm{H}), 4.71(\mathrm{~s}, 2 \mathrm{H}), 2.64-2.50(\mathrm{~m}, 2 \mathrm{H}), 1.86(\mathrm{dd}, 3 \mathrm{H}, \mathrm{J}=1.7,6.9 \mathrm{~Hz}), 1.86-1.78(\mathrm{~m}, 1 \mathrm{H})$, 1.65-1.49 (m, 4H), $0.92(\mathrm{~s}, 9 \mathrm{H}), 0.89(\mathrm{~s}, 3 \mathrm{H}), 0.87(\mathrm{~s}, 3 \mathrm{H}), 0.08(\mathrm{~s}, 6 \mathrm{H}) .{ }^{13} \mathrm{C}$ NMR $(100$ $\left.\mathrm{MHz} ; \mathrm{CDCl}_{3}\right): \delta 166.7,144.5,139.3,138.9,129.0,127.2,127.1,126.1,123.3,78.0$, 63.0, 32.1, 31.8, 31.5, 26.7, 26.2, 18.8, 18.6, 18.2, 17.9, -5.0. LRMS (ESI) $\mathrm{m} / \mathrm{z}$ calc'd for $\mathrm{C}_{24} \mathrm{H}_{40} \mathrm{O}_{3} \mathrm{SiNa}[\mathrm{M}+\mathrm{Na}]^{+}$: 427.32 ; found: 427.2 .

\section{But-2-enoic acid 4-(2-hydroxymethylphenyl)-1-isopropylbutyl ester (8c):}<smiles>CC=CC(=O)OC(CCCc1ccccc1COC(C)(C)C)C(C)C</smiles>

8b

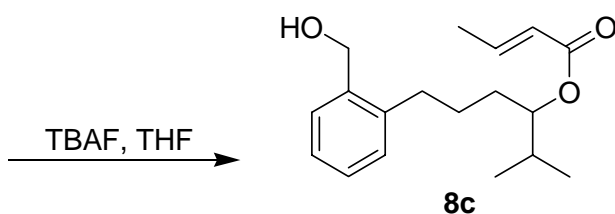

8c

Compound 8c was prepared from $\mathbf{8 b}$ by following the procedure for the preparation of $\mathbf{1 d}$ and was purified by flash chromatography (10:1 hexanes:EtOAc) to provide a colorless oil $(0.55 \mathrm{~g}, 99 \%)$.

${ }^{1} \mathrm{H}$ NMR $\left(400 \mathrm{MHz} ; \mathrm{CDCl}_{3}\right): \delta 7.35-7.32(\mathrm{~m}, 1 \mathrm{H}), 7.25-7.13(\mathrm{~m}, 3 \mathrm{H}), 6.94(\mathrm{dd}$, $1 \mathrm{H}, \mathrm{J}=6.9,15.5 \mathrm{~Hz}), 5.83(\mathrm{dq}, 1 \mathrm{H}, \mathrm{J}=1.7,15.5 \mathrm{~Hz}), 4.88-4.82(\mathrm{~m}, 1 \mathrm{H}), 4.72-4.62(\mathrm{~m}$, 
$2 \mathrm{H}), 2.77-2.56(\mathrm{~m}, 2 \mathrm{H}), 1.85(\mathrm{dd}, 3 \mathrm{H}, \mathrm{J}=1.7,6.9 \mathrm{~Hz}), 1.85-1.75(\mathrm{~m}, 2 \mathrm{H}), 1.69-1.48(\mathrm{~m}$, $4 \mathrm{H}), 0.88(\mathrm{~s}, 3 \mathrm{H}), 0.86(\mathrm{~s}, 3 \mathrm{H}) .{ }^{13} \mathrm{C}$ NMR $\left(100 \mathrm{MHz} ; \mathrm{CDCl}_{3}\right): \delta 166.9,144.8,140.6$, $138.5,129.6,128.6,128.2,126.4,123.2,77.8,63.3,32.3,31.9,31.5,27.3,18.8,18.2$, 17.8. LRMS (ESI) $\mathrm{m} / \mathrm{z}$ calc'd for $\mathrm{C}_{18} \mathrm{H}_{26} \mathrm{O}_{3} \mathrm{Na}[\mathrm{M}+\mathrm{Na}]^{+}$: 313.2 ; found: 313.1 .

\section{But-2-enoic acid 4-(2-formylphenyl)-1-isopropyllbutyl ester (8d):}
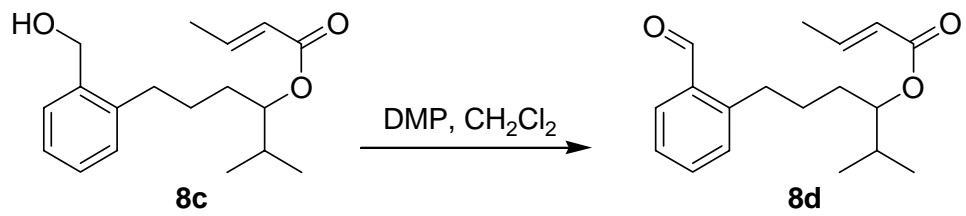

Compound 8d was prepared from 8c by following the procedure for the preparation of $\mathbf{4 a}$ and was purified by flash chromatography (20:1 hexanes:EtOAc) to provide a colorless oil $(0.42 \mathrm{~g}, 85 \%)$.

${ }^{1} \mathrm{H}$ NMR $\left.\left(400 \mathrm{MHz} ; \mathrm{CDCl}_{3}\right): \delta 10.22(\mathrm{~s}, 1 \mathrm{H}), 7.79(\mathrm{dd}, 1 \mathrm{H}), \mathrm{J}=1.5,7.7 \mathrm{~Hz}\right)$, $7.47(\mathrm{dt}, 1 \mathrm{H}, \mathrm{J}=1.5,7.5 \mathrm{~Hz}), 7.34(\mathrm{dt}, 1 \mathrm{H}, \mathrm{J}=1.0,7.5 \mathrm{~Hz}), 7.23(\mathrm{~d}, 1 \mathrm{H}, \mathrm{J}=6.0 \mathrm{~Hz}), 6.93$ $(\mathrm{dq}, 1 \mathrm{H}, \mathrm{J}=7.0,15.6 \mathrm{~Hz}), 5.82(\mathrm{dq}, 1 \mathrm{H}, \mathrm{J}=1.7,15.5 \mathrm{~Hz}), 4.86-4.80(\mathrm{~m}, 1 \mathrm{H}), 3.08-2.93$ $(\mathrm{m}, 2 \mathrm{H}), 1.86(\mathrm{dd}, 3 \mathrm{H}, \mathrm{J}=1.7,7.0 \mathrm{~Hz}), 1.85-1.75(\mathrm{~m}, 1 \mathrm{H}), 1.68-1.50(\mathrm{~m}, 4 \mathrm{H}), 0.88(\mathrm{~s}$, $3 \mathrm{H}), 0.86(\mathrm{~s}, 3 \mathrm{H}) .{ }^{13} \mathrm{C}$ NMR $\left(100 \mathrm{MHz} ; \mathrm{CDCl}_{3}\right): \delta 192.6,166.7,145.3,144.7,134.0$, $133.9,132.1,131.2,126.8,123.2,77.8,32.6,31.8,31.3,28.3,18.8,18.2,17.8 . \operatorname{IR}\left(\mathrm{cm}^{-1}\right)$ : 2964, 2875, 1714, 1695, 1658, 1292, 1266, 1188, 754. HRMS (ESI) $\mathrm{m} / \mathrm{z}$ calc'd for $\mathrm{C}_{18} \mathrm{H}_{24} \mathrm{O}_{3} \mathrm{Na}[\mathrm{M}+\mathrm{Na}]^{+}:$311.1617; found: 311.1610 .

Scheme 8. Preparation of substrate 9e (precursor of lactone 9; Table 2, entry 7)

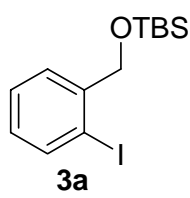

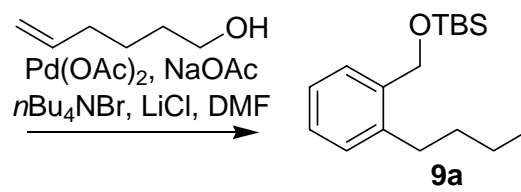
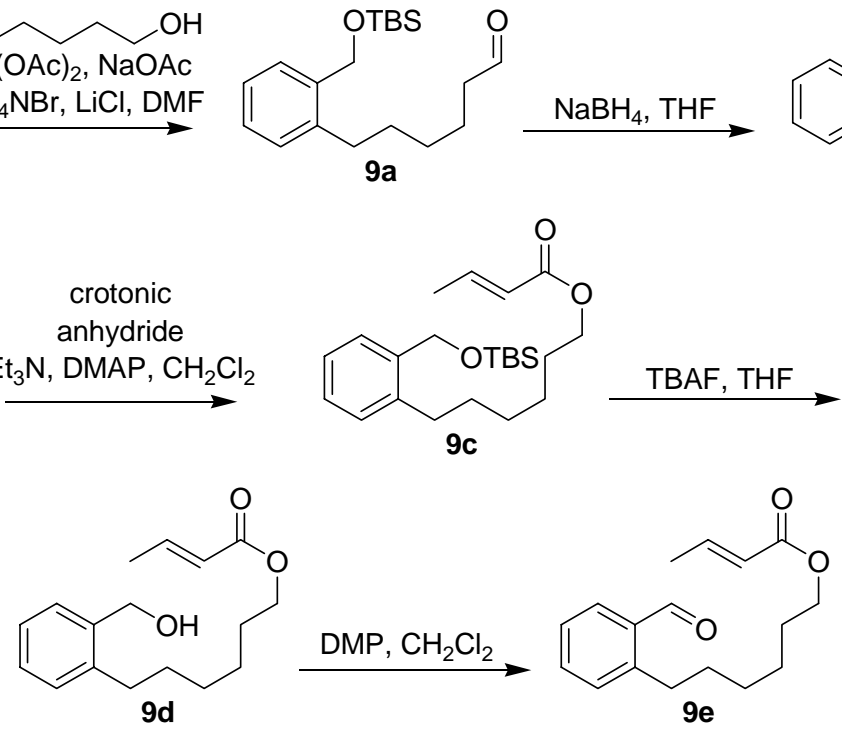

A Heck reaction between TBS ether $3 \mathrm{a}^{3}$ and 5-hexen-1-ol provided aldehyde 9a, which was reduced with sodium borohydride to afford alcohol $\mathbf{9 b}$. Acylation with crotonic anhydride followed by a TBS deprotection resulted in alcohol 9d, which was oxidized with DMP to aldehyde 9e. 


\section{6-[2-(tert-Butyldimethylsilanyloxymethyl)phenyl]hexanal (9a):}

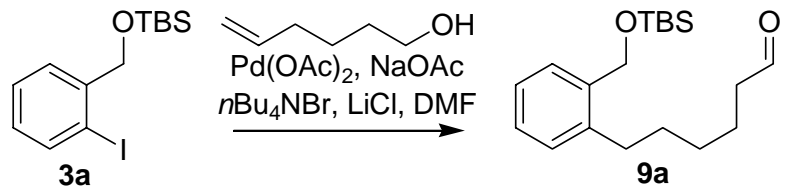

$n \mathrm{Bu}_{4} \mathrm{NBr}(25.8 \mathrm{~g}, 80 \mathrm{mmol}), \mathrm{NaOAc}(8.2 \mathrm{~g}, 100 \mathrm{mmol}), \mathrm{LiCl}(1.7 \mathrm{~g}, 40 \mathrm{mmol})$, and $\mathrm{Pd}(\mathrm{OAc})_{2}(0.9 \mathrm{~g}, 4.0 \mathrm{mmol})$ were added sequentially to a stirred solution of 3a (13.9 $\mathrm{g}, 40 \mathrm{mmol})$ and 5-hexen-1-ol (4.2 g, $42 \mathrm{mmol})$ in anhydrous DMF (80 mL). The mixture was heated to $50^{\circ} \mathrm{C}$. After 4 days at this temperature, the reaction mixture was to room temperature and diluted with EtOAc $(150 \mathrm{~mL})$ and hexanes $(300 \mathrm{~mL})$ resulting in a biphasic organic solution. The DMF-containing phase was separated and extracted with hexanes $(100 \mathrm{~mL})$. The combined hexanes/EtOAc extracts were washed with deionized water, 1.0 $\mathrm{M} \mathrm{CuSO}_{4}$, deionized water, $1.0 \mathrm{M} \mathrm{NaOH}$, deionized water, and brine. After drying over $\mathrm{MgSO}_{4}$ and concentrating under reduced pressure, the crude product was purified by flash chromatography (30:1 hexanes:EtOAc) to afford aldehyde 9a (6.3 g, $19.7 \mathrm{mmol}, 49 \%$ ).

${ }^{1} \mathrm{H}$ NMR $\left(500 \mathrm{MHz} ; \mathrm{CDCl}_{3}\right): \delta 9.75(\mathrm{t}, 1 \mathrm{H}, \mathrm{J}=1.8 \mathrm{~Hz}), 7.43-7.35(\mathrm{~m}, 1 \mathrm{H}), 7.20-$ $7.16(\mathrm{~m}, 2 \mathrm{H}), 7.13-7.10(\mathrm{~m}, 1 \mathrm{H}), 4.71(\mathrm{~s}, 2 \mathrm{H}), 2.60-2.55(\mathrm{~m}, 2 \mathrm{H}), 1.69-1.56(\mathrm{~m}, 4 \mathrm{H})$, $1.43-1.36(\mathrm{~m}, 2 \mathrm{H}), 0.92(\mathrm{~s}, 9 \mathrm{H}), 0.08(\mathrm{~s}, 6 \mathrm{H}) .{ }^{13} \mathrm{C} \mathrm{NMR}\left(100 \mathrm{MHz} ; \mathrm{CDCl}_{3}\right): \delta 202.8$, 139.7, 138.8, 129.0, 127.4, 127.3, 126.1, 63.2, 44.1, 32.1, 30.7, 29.4, 26.2, 22.2, 18.6, 5.0. LRMS (ESI) $\mathrm{m} / \mathrm{z}$ calc'd for $\mathrm{C}_{19} \mathrm{H}_{32} \mathrm{O}_{2} \mathrm{SiNa}[\mathrm{M}+\mathrm{Na}]^{+}$: 343.2; found: 343.2 .

\section{6-[2-(tert-Butyldimethylsilanyloxymethyl)phenyl]hexan-1-ol (9b):}

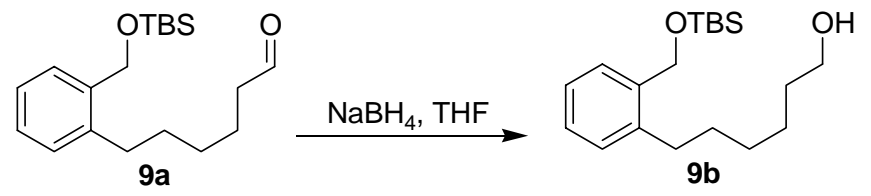

Compound 9b was prepared from 9a according to the procedure for the preparation of $\mathbf{6 b}$ and was purified by flash chromatography (7:1 hexanes:EtOAc) to provide a colorless oil $(1.11 \mathrm{~g}, 65 \%)$.

${ }^{1} \mathrm{H}$ NMR $\left(400 \mathrm{MHz} ; \mathrm{CDCl}_{3}\right): \delta$ 7.45-7.40 (m, $\left.1 \mathrm{H}\right), 7.22-7.12(\mathrm{~m}, 3 \mathrm{H}), 4.75(\mathrm{~s}$, $2 \mathrm{H}), 3.62(\mathrm{t}, 2 \mathrm{H}, \mathrm{J}=6.6 \mathrm{~Hz}), 2.62-2.56(\mathrm{~m}, 2 \mathrm{H}), 1.64-1.50(\mathrm{~m}, 5 \mathrm{H}), 1.45-1.36(\mathrm{~m}, 4 \mathrm{H})$, $0.95(\mathrm{~s}, 9 \mathrm{H}), 0.11(\mathrm{~s}, 6 \mathrm{H}) .{ }^{13} \mathrm{C} \mathrm{NMR}\left(100 \mathrm{MHz} ; \mathrm{CDCl}_{3}\right): \delta 139.9,138.8,129.0,127.2$, 127.1, 126.0, 63.1, 32.9, 32.3, 30.9, 29.7, 26.2, 25.9, 18.6, -5.0. LRMS (ESI) $\mathrm{m} / \mathrm{z}$ calc'd for $\mathrm{C}_{19} \mathrm{H}_{34} \mathrm{O}_{2} \mathrm{SiNa}[\mathrm{M}+\mathrm{Na}]^{+}$: 345.2; found: 345.1 .

But-2-enoic acid 6-[2-(tert-Butyldimethylsilanyloxymethyl)phenyl]hexyl ester (9c):

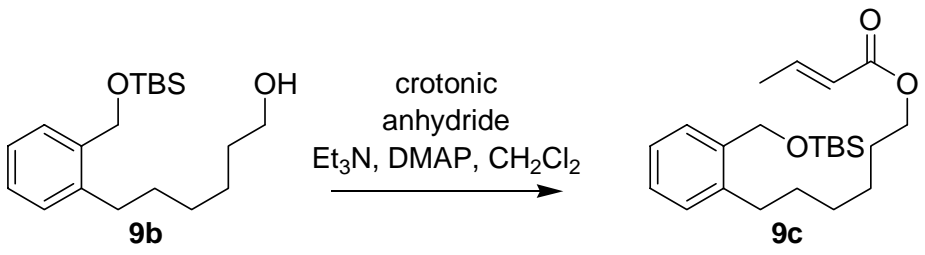


Compound 9c was prepared from 9b by following the procedure for the preparation of 1c and was purified by flash chromatography (15:1 hexanes:EtOAc) to provide a colorless oil $(0.93 \mathrm{~g}, 71 \%)$.

${ }^{1} \mathrm{H}$ NMR $\left(400 \mathrm{MHz} ; \mathrm{CDCl}_{3}\right): \delta 7.43-7.38(\mathrm{~m}, 1 \mathrm{H}), 7.21-7.10(\mathrm{~m}, 3 \mathrm{H}), 6.96(\mathrm{dq}$, $1 \mathrm{H}, \mathrm{J}=7.0,15.6 \mathrm{~Hz}), 5.84(\mathrm{dq}, 1 \mathrm{H}, \mathrm{J}=1.7,15.6 \mathrm{~Hz}), 4.73(\mathrm{~s}, 2 \mathrm{H}), 4.10(\mathrm{t}, 2 \mathrm{H}, \mathrm{J}=6.7$ $\mathrm{Hz}), 2.60-2.54(\mathrm{~m}, 2 \mathrm{H}), 1.86(\mathrm{dd}, 3 \mathrm{H}, \mathrm{J}=1.7,7.0 \mathrm{~Hz}), 1.70-1.54(\mathrm{~m}, 4 \mathrm{H}), 1.44-1.34(\mathrm{~m}$, $4 \mathrm{H}), 0.93(\mathrm{~s}, 9 \mathrm{H}), 0.09(\mathrm{~s}, 6 \mathrm{H}) .{ }^{13} \mathrm{C} \mathrm{NMR}\left(100 \mathrm{MHz} ; \mathrm{CDCl}_{3}\right): \delta 166.9,144.7,139.9$, $138.8,129.0,127.3,127.2,126.1,123.0,68.2,64.5,63.1,32.3,30.9,29.6,28.9,26.2$, 26.1, 18.7, 18.2, -5.0. LRMS (ESI) $\mathrm{m} / \mathrm{z}$ calc'd for $\mathrm{C}_{23} \mathrm{H}_{38} \mathrm{O}_{3} \mathrm{SiNa}[\mathrm{M}+\mathrm{Na}]^{+}:$413.6; found: 413.1 .

\section{But-2-enoic acid 6-(2-hydroxymethylphenyl)hexyl ester (9d):}

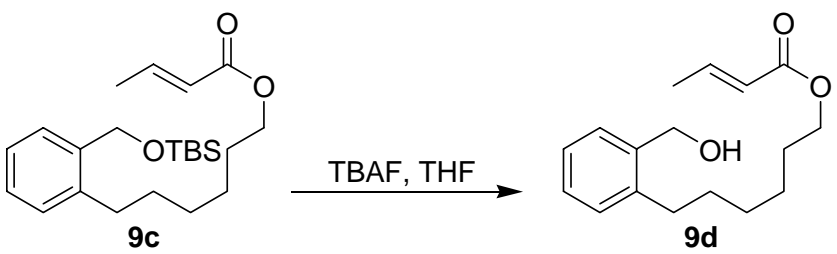

Compound 9d was prepared from 9c by following the procedure for the preparation of 1d and was purified by flash chromatography (10:1 hexanes:EtOAc) to provide a colorless oil $(0.53 \mathrm{~g}, 88 \%)$.

${ }^{1} \mathrm{H}$ NMR $\left(400 \mathrm{MHz} ; \mathrm{CDCl}_{3}\right): \delta$ 7.37-7.33 (m, 1H), 7.23-7.15 (m, 3H), $6.94(\mathrm{dq}$, $1 \mathrm{H}, \mathrm{J}=6.9,15.5 \mathrm{~Hz}), 5.82(\mathrm{dq}, 1 \mathrm{H}, \mathrm{J}=1.7,15.5 \mathrm{~Hz}), 4.70(\mathrm{~d}, 2 \mathrm{H}, \mathrm{J}=5.7 \mathrm{~Hz}), 4.09(\mathrm{t}$, $2 \mathrm{H}, \mathrm{J}=6.7 \mathrm{~Hz}), 2.68-2.62(\mathrm{~m}, 2 \mathrm{H}), 1.86(\mathrm{dd}, 3 \mathrm{H}, \mathrm{J}=1.7,6.9 \mathrm{~Hz}), 1.68-1.55(\mathrm{~m}, 4 \mathrm{H})$, $1.57(\mathrm{t}, 1 \mathrm{H}, \mathrm{J}=5.7 \mathrm{~Hz}), 1.44-1.35(\mathrm{~m}, 4 \mathrm{H}) .{ }^{13} \mathrm{C} \mathrm{NMR}\left(100 \mathrm{MHz} ; \mathrm{CDCl}_{3}\right): \delta 167.0$, 144.8, 141.0, 138.4, 129.6, 128.4, 128.2, 126.4, 123.0, 64.5, 63.4, 32.5, 31.4, 29.5, 28.9, 26.1, 18.2. LRMS (ESI) $\mathrm{m} / \mathrm{z}$ calc'd for $\mathrm{C}_{17} \mathrm{H}_{24} \mathrm{O}_{3} \mathrm{Na}[\mathrm{M}+\mathrm{Na}]^{+}:$299.4; found: 299.1 .

\section{But-2-enoic acid 6-(2-formylphenyl)hexyl ester (9e):}

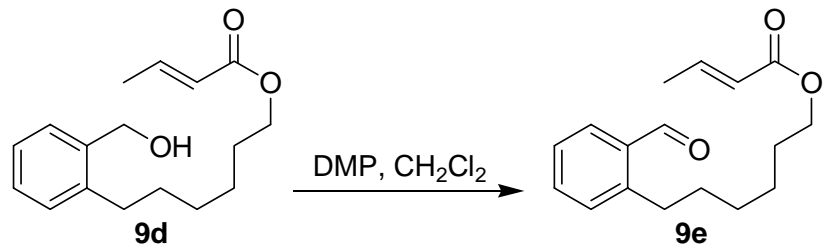

Compound 9e was prepared from 9d by following the procedure for the preparation of $\mathbf{4 a}$ and was purified by flash chromatography (20:1 hexanes:EtOAc) to provide a colorless oil $(0.47 \mathrm{~g}, 91 \%)$.

${ }^{1} \mathrm{H}$ NMR (400 MHz; $\left.\mathrm{CDCl}_{3}\right): \delta 10.25(\mathrm{~s}, 1 \mathrm{H}), 7.80(\mathrm{dd}, 1 \mathrm{H}, \mathrm{J}=1.4,7.6 \mathrm{~Hz}), 7.47$ $(\mathrm{dt}, 1 \mathrm{H}, \mathrm{J}=1.5,7.4 \mathrm{~Hz}), 7.34(\mathrm{dt}, 1 \mathrm{H}, \mathrm{J}=1.1,7.5 \mathrm{~Hz}), 7.26-7.22(\mathrm{~m}, 1 \mathrm{H}), 6.94(\mathrm{dq}, 1 \mathrm{H}, \mathrm{J}$ $=6.9,15.5 \mathrm{~Hz}), 5.81(\mathrm{dq}, 1 \mathrm{H}, \mathrm{J}=1.7,15.5 \mathrm{~Hz}), 4.08(\mathrm{t}, 2 \mathrm{H}, \mathrm{J}=6.6 \mathrm{~Hz}), 3.03-2.96(\mathrm{~m}$, $2 \mathrm{H}), 1.85(\mathrm{dd}, 3 \mathrm{H}, \mathrm{J}=1.7,7.0 \mathrm{~Hz}), 1.66-1.54(\mathrm{~m}, 4 \mathrm{H}), 1.45-1.33(\mathrm{~m}, 4 \mathrm{H}) .{ }^{13} \mathrm{C} \mathrm{NMR}$ $\left(100 \mathrm{MHz} ; \mathrm{CDCl}_{3}\right): \delta 192.6,166.9,145.8,144.7,134.0,133.9,132.0,131.2,126.7$, 123.0, 64.4, 32.7, 32.4, 29.4, 28.8, 26.0, 18.2. IR ( $\left.\mathrm{cm}^{-1}\right): 2935,2858,2740,1718,1698$, $1659,1312,1268,1186,756$. HRMS (ESI) $m / z$ calc'd for $\mathrm{C}_{17} \mathrm{H}_{23} \mathrm{O}_{3}[\mathrm{M}+\mathrm{H}]^{+}:$: 275.1641; found: 275.1634 . 
Scheme 9. Preparation of substrate 10d (precursor of lactone 10; Table 2, entry 8)

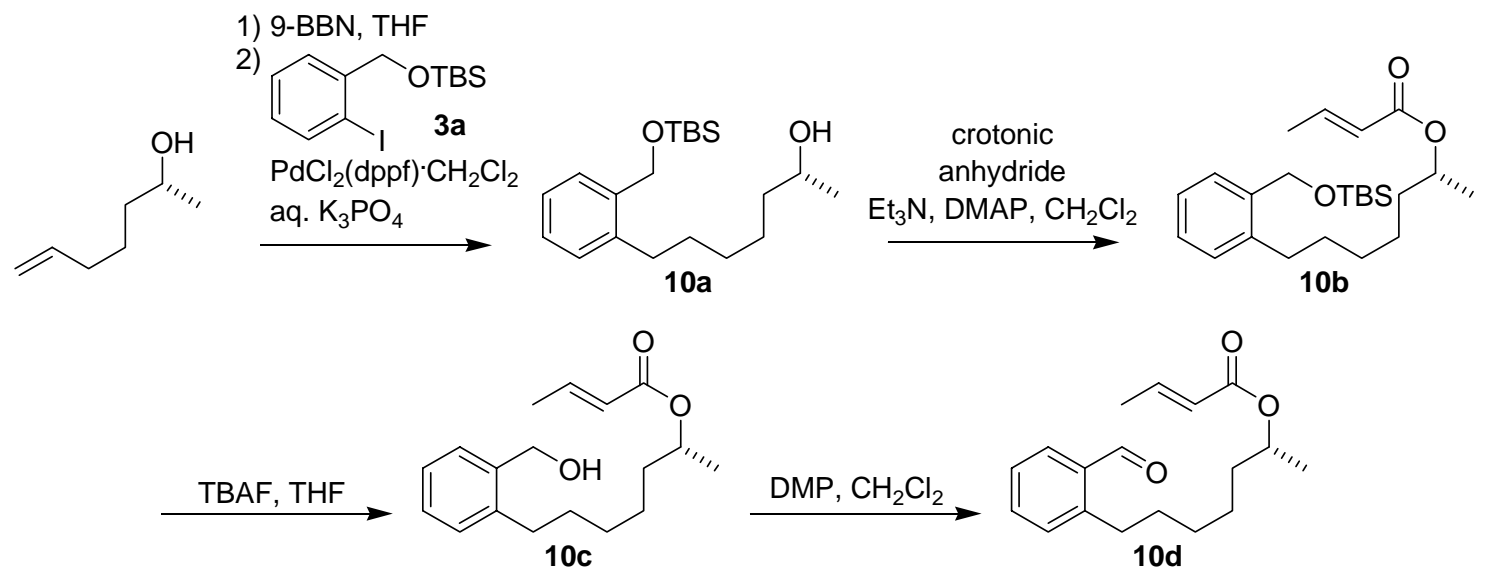

The Suzuki cross-coupling reaction between the hydroborated intermediate obtained from $(R)-6$-hepten-2-ol ${ }^{6}$ and the TBS-ether $3 \mathbf{a}^{3}$ provided alcohol 10a, which was acylated with crotonic anhydride to afford ester 10b. Treatment with TBAF resulted in alcohol 10c, which was oxidized to the aldehyde 10d with DMP.

\section{7-[2-(tert-Butyldimethylsilanyloxymethyl)phenyl]heptan-2-ol (10a):}

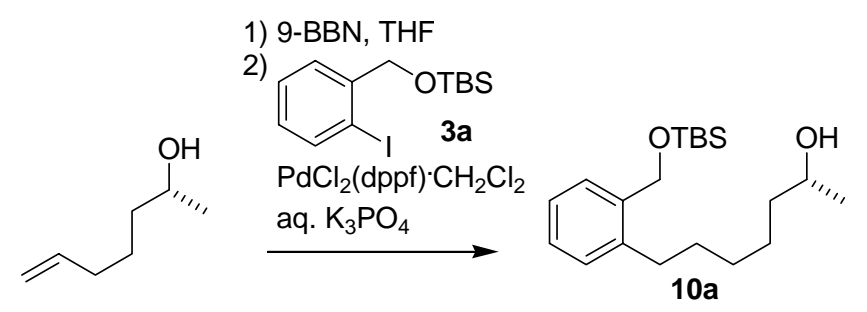

9-BBN (44.3 mL, $22.2 \mathrm{mmol}, 0.5 \mathrm{M}$ in THF) was added to $(R)-6$-hepten-2-ol ${ }^{6}$ $(1.15 \mathrm{~g}, 10.1 \mathrm{mmol})$ at $0^{\circ} \mathrm{C}$. The solution was allowed to warm to room temperature and stirred for $2 \mathrm{hr}$. A solution of $\mathrm{K}_{3} \mathrm{PO}_{4}\left(14.7 \mathrm{~mL}, 44.3 \mathrm{mmol}, 3 \mathrm{M}\right.$ in $\left.\mathrm{H}_{2} \mathrm{O}\right)$, a solution of TBS-ether $3 \mathrm{a}^{3}$ (3.94 g, $\left.11.3 \mathrm{mmol}\right)$ in THF $(4 \mathrm{~mL})$, and $\mathrm{PdCl}_{2}(\mathrm{dppf}) \mathrm{CH}_{2} \mathrm{Cl}_{2}(0.8 \mathrm{~g}, 1$ mmol) were added sequentially, and the mixture was allowed to stir at room temperature overnight $(\sim 16 \mathrm{hr}) . \mathrm{Et}_{2} \mathrm{O}(20 \mathrm{~mL})$ was added and the mixture was washed with saturated $\mathrm{NaHCO}_{3}$ and brine, dried over anhydrous $\mathrm{MgSO}_{4}$, and concentrated under reduced pressure on the rotary evaporator. The crude product was dissolved in THF $(150 \mathrm{~mL})$, cooled to $0^{\circ} \mathrm{C}$, and a solution of $\mathrm{NaOH}(50 \mathrm{~mL}, 1 \mathrm{M})$ and aqueous $\mathrm{H}_{2} \mathrm{O}_{2}(5 \mathrm{~mL}, 30 \%)$ were added sequentially. The mixture was vigorously stirred for $30 \mathrm{~min}$, diluted with $\mathrm{Et}_{2} \mathrm{O}(150 \mathrm{~mL})$, then washed with saturated $\mathrm{NaHCO}_{3}$ and brine, dried over anhydrous $\mathrm{MgSO}_{4}$, and concentrated under reduced pressure on the rotary evaporator. Purification was achieved by flash chromatography (10:1 hexanes:EtOAc) to provide the alcohol 10a $(2.73 \mathrm{~g}, 81 \%)$.

${ }^{1} \mathrm{H}$ NMR (400 MHz; $\left.\mathrm{CDCl}_{3}\right): \delta$ 7.43-7.38 (m, $\left.1 \mathrm{H}\right), 7.21-7.10(\mathrm{~m}, 3 \mathrm{H})$, 4.73 (s, 2H), 3.82-3.73 (br m, 1H), 2.60-2.54 (m, 2H), 1.63-1.54 (m, 2H), 1.50-1.32 (m, $6 \mathrm{H}), 1.30$ (br s, 1H), $1.17(\mathrm{~d}, 3 \mathrm{H}, \mathrm{J}=6.2 \mathrm{~Hz}), 0.93(\mathrm{~s}, 9 \mathrm{H}), 0.09(\mathrm{~s}, 6 \mathrm{H}) .{ }^{13} \mathrm{C} \mathrm{NMR}(100$

\footnotetext{
${ }^{6}$ Furstner, A.; Thiel, O. R.; Ackermann, L. Org. Lett. 2001, 3, 449.
} 
$\left.\mathrm{MHz} ; \mathrm{CDCl}_{3}\right): \delta 139.9,138.8,129.0,127.2,126.0,68.4,63.1,39.5,32.3,30.9,30.0$, 26.2, 26.0, 23.8, 18.7, -5.0. [ $\alpha]_{\mathrm{D}}-6.7\left(c \mathrm{c} 0.99,26^{\circ} \mathrm{C}, \mathrm{CH}_{2} \mathrm{Cl}_{2}\right)$. LRMS (ESI) $\mathrm{m} / \mathrm{z}$ calc'd for $\mathrm{C}_{20} \mathrm{H}_{36} \mathrm{O}_{2} \mathrm{SiNa}[\mathrm{M}+\mathrm{Na}]^{+}: 359.2$; found: 359.2 .

\section{But-2-enoic acid 6-[2-(tert-Butyldimethylsilanyloxymethyl)phenyl]-1-methylhexyl ester (10b):}

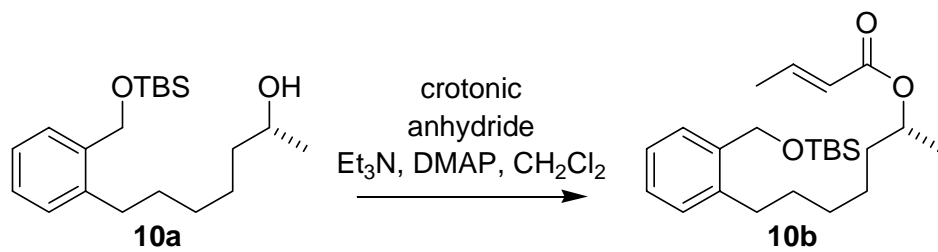

Compound 10b was prepared from 10a by following the procedure for the preparation of 1c and was purified by flash chromatography (15:1 hexanes:EtOAc) to provide a colorless oil $(1.32 \mathrm{~g}, 84 \%)$.

${ }^{1} \mathrm{H}$ NMR $\left(500 \mathrm{MHz} ; \mathrm{CDCl}_{3}\right): \delta$ 7.42-7.38 (m, 1H), 7.19-7.15 (m, 2H), 7.13-7.09 $(\mathrm{m}, 1 \mathrm{H}), 6.93(\mathrm{dq}, 1 \mathrm{H}, \mathrm{J}=6.9,15.5 \mathrm{~Hz}), 5.80(\mathrm{dq}, 1 \mathrm{H}, \mathrm{J}=1.8,15.5 \mathrm{~Hz}), 4.97-4.89(\mathrm{~m}$, $1 \mathrm{H}), 4.71(\mathrm{~s}, 2 \mathrm{H}), 2.58-2.52(\mathrm{~m}, 2 \mathrm{H}), 1.85(\mathrm{dd}, 3 \mathrm{H}, \mathrm{J}=1.8,6.9 \mathrm{~Hz}), 1.64-1.26(\mathrm{~m}, 8 \mathrm{H})$, $1.20(\mathrm{~d}, 3 \mathrm{H}, \mathrm{J}=6.3 \mathrm{~Hz}), 0.92(\mathrm{~s}, 9 \mathrm{H}), 0.08(\mathrm{~s}, 6 \mathrm{H}) .{ }^{13} \mathrm{C}$ NMR $\left(100 \mathrm{MHz} ; \mathrm{CDCl}_{3}\right): \delta$ $166.5,144.4,139.9,138.8,129.0,127.2$, 126.0, 123.5, 70.9, 63.1, 36.2, 33.0, 30.9, 29.8, 26.2, 25.6, 20.3, 18.7, 18.2, -5.0. [ $\alpha]_{\mathrm{D}}-8.1$ (c $0.70,26^{\circ} \mathrm{C}, \mathrm{CH}_{2} \mathrm{Cl}_{2}$ ). LRMS (ESI) $\mathrm{m} / \mathrm{z}$ calc'd for $\mathrm{C}_{24} \mathrm{H}_{40} \mathrm{O}_{3} \mathrm{SiNa}[\mathrm{M}+\mathrm{Na}]^{+}$: 427.3; found: 427.2 .

\section{But-2-enoic acid 6-(2-hydroxymethylphenyl)-1-methylhexyl ester (10c):}

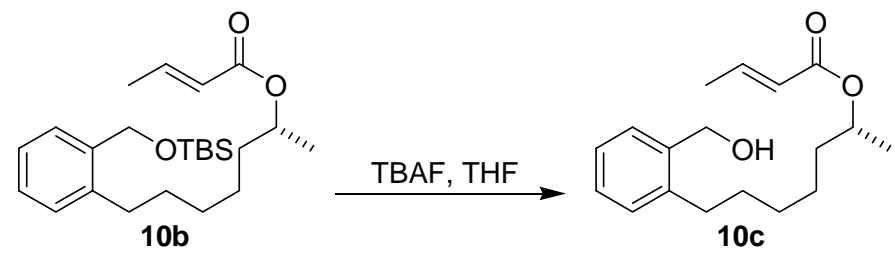

Compound 10c was prepared from 10b by following the procedure for the preparation of 1d and was purified by flash chromatography (5:1 hexanes:EtOAc) to provide a colorless oil $(0.74 \mathrm{~g}, 83 \%)$.

${ }^{1} \mathrm{H}$ NMR (400 MHz; $\left.\mathrm{CDCl}_{3}\right): \delta$ 7.37-7.33 (m, 1H), 7.23-7.14 (m, 3H), $6.93(\mathrm{dq}$, $1 \mathrm{H}, \mathrm{J}=6.9,15.5 \mathrm{~Hz}), 5.80(\mathrm{dq}, 1 \mathrm{H}, \mathrm{J}=1.7,15.5 \mathrm{~Hz}), 4.97-4.88(\mathrm{~m}, 1 \mathrm{H}), 4.70(\mathrm{~s}, 2 \mathrm{H})$, 2.67-2.61 (m, 2H), $1.85(\mathrm{dd}, 3 \mathrm{H}, \mathrm{J}=1.7,7.0 \mathrm{~Hz}), 1.65-1.26(\mathrm{~m}, 9 \mathrm{H}), 1.20(\mathrm{~d}, 3 \mathrm{H}, \mathrm{J}=6.2$ $\mathrm{Hz}) .{ }^{13} \mathrm{C} \mathrm{NMR}\left(100 \mathrm{MHz} ; \mathrm{CDCl}_{3}\right): \delta 166.6,144.5,141.0,138.5,129.6,128.4,128.2$, $126.4,123.5,70.9,63.4,36.1,32.5,31.4,29.7,25.5,20.3,18.2,15.9$. [ $\alpha]_{\mathrm{D}}-4.9$ (c 0.75 , $26^{\circ} \mathrm{C}, \mathrm{CH}_{2} \mathrm{Cl}_{2}$ ). LRMS (ESI) $\mathrm{m} / \mathrm{z}$ calc'd for $\mathrm{C}_{18} \mathrm{H}_{26} \mathrm{O}_{3} \mathrm{Na}[\mathrm{M}+\mathrm{Na}]^{+}$: 313.2 ; found: 313.1 . 
But-2-enoic acid 6-(2-formylphenyl)-1-methylhexyl ester (10d):

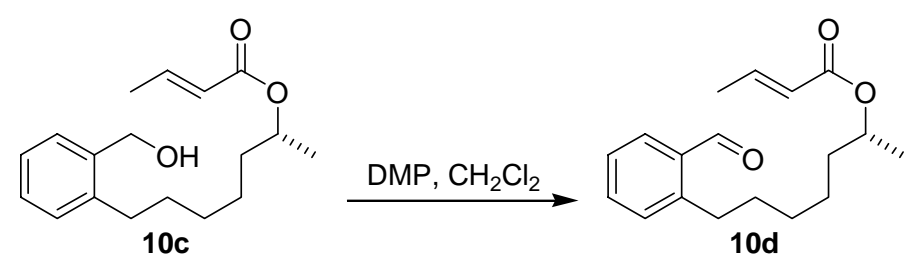

Compound 10d was prepared from 10c by following the procedure for the preparation of 4a and was purified by flash chromatography (15:1 hexanes:EtOAc) to provide a colorless oil $(0.53 \mathrm{~g}, 77 \%)$.

${ }^{1} \mathrm{H}$ NMR $\left(400 \mathrm{MHz} ; \mathrm{CDCl}_{3}\right): \delta 10.24(\mathrm{~s}, 1 \mathrm{H}), 7.79(\mathrm{dd}, 1 \mathrm{H}, \mathrm{J}=1.5,7.7 \mathrm{~Hz}), 7.46$ $(\mathrm{dt}, 1 \mathrm{H}, \mathrm{J}=1.5,7.5 \mathrm{~Hz}), 7.32(\mathrm{dt}, 1 \mathrm{H}, \mathrm{J}=1.1,7.5 \mathrm{~Hz}), 7.22(\mathrm{dd}, 1 \mathrm{H}, \mathrm{J}=0.7,7.7 \mathrm{~Hz})$, $6.91(\mathrm{dq}, 1 \mathrm{H}, \mathrm{J}=6.9,15.5 \mathrm{~Hz}), 5.78(\mathrm{dq}, 1 \mathrm{H}, \mathrm{J}=1.7,15.5 \mathrm{~Hz}), 4.95-4.86(\mathrm{~m}, 1 \mathrm{H}), 3.01$ $2.94(\mathrm{~m}, 2 \mathrm{H}), 1.83(\mathrm{dd}, 3 \mathrm{H}, \mathrm{J}=1.7,7.0 \mathrm{~Hz}), 1.63-1.26(\mathrm{~m}, 8 \mathrm{H}), 1.18(\mathrm{~d}, 3 \mathrm{H}, \mathrm{J}=6.2 \mathrm{~Hz})$. ${ }^{13} \mathrm{C}$ NMR $\left(100 \mathrm{MHz} ; \mathrm{CDCl}_{3}\right): \delta 192.5,166.4,145.8,144.3,133.9,133.8,131.8,131.2$, 126.6, 123.4, 70.7, 36.1, 32.6, 32.4, 29.5, 25.4, 20.2, 18.1. IR ( $\left.\mathrm{cm}^{-1}\right): 2936,2860,1715$, 1696, 1660, 1293, 1190, 1102, 754. $[\alpha]_{\mathrm{D}}-12.1\left(c 0.92,26^{\circ} \mathrm{C}, \mathrm{CH}_{2} \mathrm{Cl}_{2}\right)$. HRMS (ESI) $\mathrm{m} / \mathrm{z}$ calc'd for $\mathrm{C}_{18} \mathrm{H}_{24} \mathrm{O}_{3} \mathrm{Na}[\mathrm{M}+\mathrm{Na}]^{+}$: 311.1617 ; found: 311.1623 .

Scheme 10. Preparation of substrate 11d (precursor of lactone 11; Table 2, entry 9)

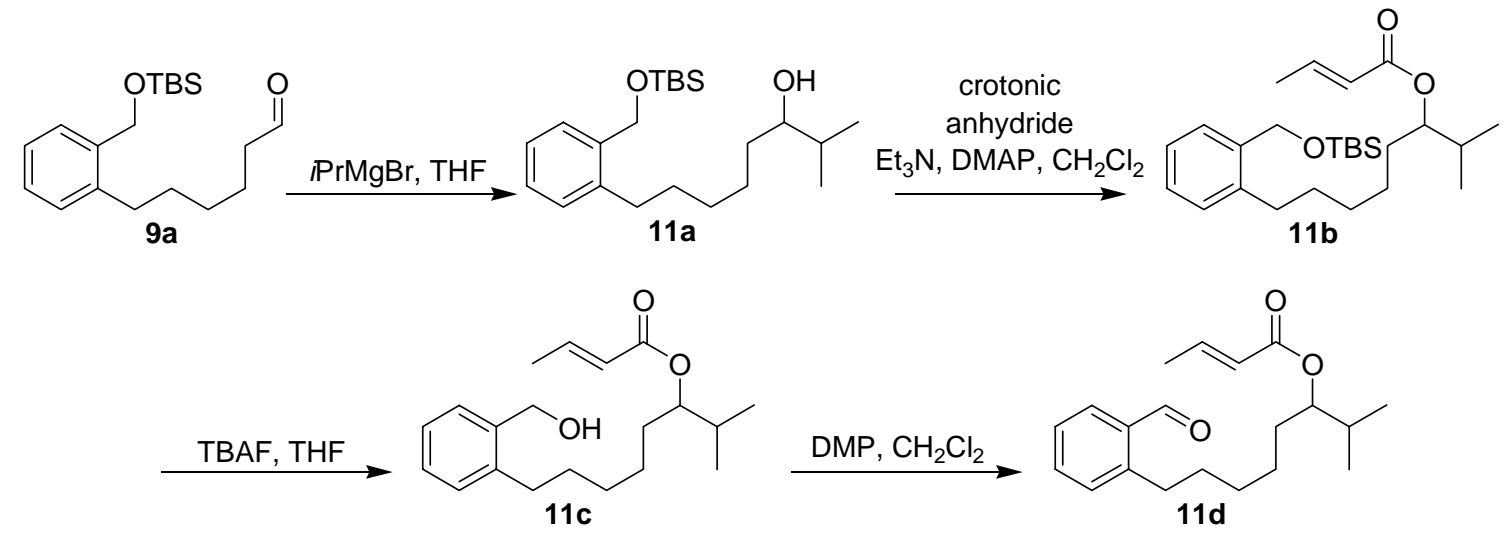

Treatment of aldehyde 9a with isopropylmagnesium bromide resulted in alcohol 11a, which was acylated with crotonic anhydride to afford ester 11b. The deprotection of the TBS group was achieved with TBAF to provide alcohol 11c, which was oxidized to aldehyde 11d with DMP.

\section{8-[2-(tert-Butyldimethylsilanyloxymethyl)phenyl]-2-methyloctan-3-ol (11a):}

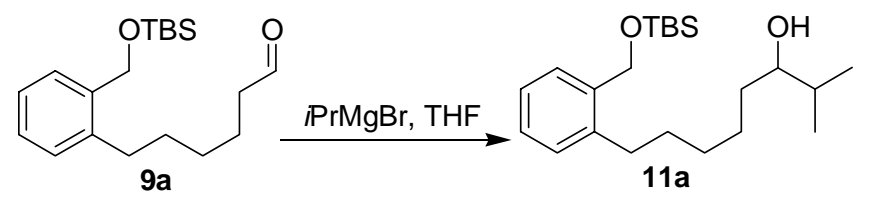

Compound 11a was prepared from 9a according to the procedure for the preparation of $\mathbf{5 a}$ and was purified by flash chromatography (10:1 hexanes:EtOAc) to provide a colorless oil $(1.73 \mathrm{~g}, 75 \%)$. 
${ }^{1} \mathrm{H}$ NMR $\left(400 \mathrm{MHz} ; \mathrm{CDCl}_{3}\right): \delta$ 7.46-7.40 (m, 1H), 7.22-7.12 (m, 3H), $4.75(\mathrm{~s}$, 2H), 3.39-3.32 (m, 1H), 2.63-2.55 (m, 2H), 1.69-1.32 (m, 8H), $0.95(\mathrm{~s}, 9 \mathrm{H}), 0.92(\mathrm{~d}, 3 \mathrm{H}$, $\mathrm{J}=4.6 \mathrm{~Hz}), 0.90(\mathrm{~d}, 3 \mathrm{H}, \mathrm{J}=4.5 \mathrm{~Hz}), 0.11(\mathrm{~s}, 6 \mathrm{H}) .{ }^{13} \mathrm{C} \mathrm{NMR}\left(100 \mathrm{MHz} ; \mathrm{CDCl}_{3}\right): \delta$ $139.9,138.8,129.0,127.2$, 127.1, 126.0, 76.9, 63.1, 34.3, 33.7, 32.3, 31.0, 30.1, 26.2, 19.1, 18.6, 17.3, -5.0. LRMS (ESI) $\mathrm{m} / \mathrm{z}$ calc'd for $\mathrm{C}_{22} \mathrm{H}_{40} \mathrm{O}_{2} \mathrm{SiNa}[\mathrm{M}+\mathrm{Na}]^{+}:$387.6; found: 387.1 .

But-2-enoic acid 6-[2-(tert-Butyldimethylsilanyloxymethyl)phenyl]-1-isopropylhexyl ester (11b):

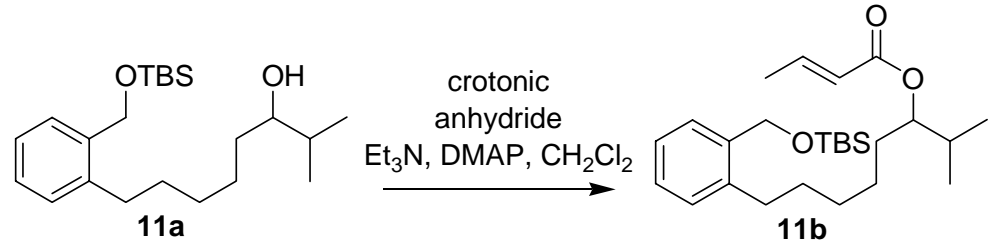

Compound 11b was prepared from 11a by following the procedure for the preparation of 1c and was purified by flash chromatography (20:1 hexanes:EtOAc) to provide a colorless oil $(1.49 \mathrm{~g}, 81 \%)$.

${ }^{1} \mathrm{H}$ NMR $\left(500 \mathrm{MHz} ; \mathrm{CDCl}_{3}\right): \delta$ 7.44-7.38 (m, 1H), 7.19-7.03 (m, 3H), $6.94(\mathrm{dq}$, $1 \mathrm{H}, \mathrm{J}=6.9,15.5 \mathrm{~Hz}), 5.83(\mathrm{dq}, 1 \mathrm{H}, \mathrm{J}=1.8,15.5 \mathrm{~Hz}), 4.80-4.75(\mathrm{~m}, 1 \mathrm{H}), 4.71(\mathrm{~s}, 2 \mathrm{H})$, 2.57-2.50 (m, 2H), 1.86-1.75 (m, 1H), $1.85(\mathrm{dd}, 3 \mathrm{H}, \mathrm{J}=1.8,6.9 \mathrm{~Hz}), 1.59-1.44(\mathrm{~m}, 4 \mathrm{H})$, 1.43-1.22 (m, 4H), $0.92(\mathrm{~s}, 9 \mathrm{H}), 0.88(\mathrm{~s}, 3 \mathrm{H}), 0.86(\mathrm{~s}, 3 \mathrm{H}), 0.08(\mathrm{~s}, 6 \mathrm{H}) .{ }^{13} \mathrm{C}$ NMR $(100$ $\left.\mathrm{MHz} ; \mathrm{CDCl}_{3}\right): \delta 166.8,144.4,139.9,138.8,129.0,127.2,127.1,126.0,123.4,78.3$, $63.1,32.3,31.7,31.4,30.8,30.2,30.0,26.2,25.8,18.8,18.7,18.2,17.9,-5.0$. LRMS (ESI) $\mathrm{m} / \mathrm{z}$ calc'd for $\mathrm{C}_{26} \mathrm{H}_{44} \mathrm{O}_{3} \mathrm{SiNa}[\mathrm{M}+\mathrm{Na}]^{+}$: 455.7; found: 456.1 .

\section{But-2-enoic acid 6-(2-hydroxymethylphenyl)-1-isopropylhexyl ester (11c):}

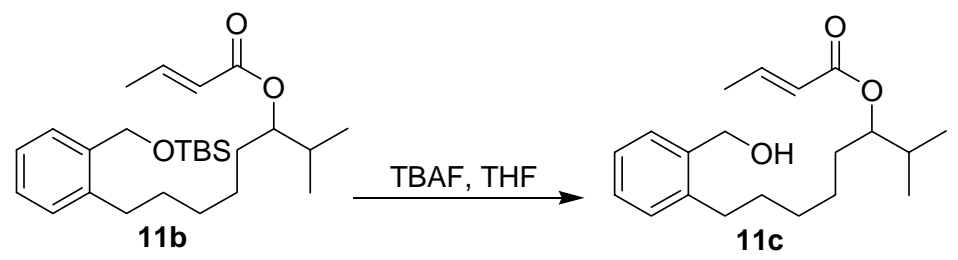

Compound 11c was prepared from 11b by following the procedure for the preparation of 1d and was purified by flash chromatography (10:1 hexanes:EtOAc) to provide a colorless oil (1.05 g, 95\%).

${ }^{1} \mathrm{H}$ NMR $\left(400 \mathrm{MHz} ; \mathrm{CDCl}_{3}\right): \delta 7.37-7.33(\mathrm{~m}, 1 \mathrm{H}), 7.24-7.13(\mathrm{~m}, 3 \mathrm{H}), 6.94(\mathrm{dq}$, $1 \mathrm{H}, \mathrm{J}=6.9,15.5 \mathrm{~Hz}), 5.82(\mathrm{dq}, 1 \mathrm{H}, \mathrm{J}=1.7,15.5 \mathrm{~Hz}), 4.77(\mathrm{dd}, 1 \mathrm{H}, \mathrm{J}=5.5,12.5 \mathrm{~Hz})$, $4.68(\mathrm{~d}, 2 \mathrm{H}, \mathrm{J}=5.6 \mathrm{~Hz}), 2.63-2.59(\mathrm{~m}, 2 \mathrm{H}), 1.88(\mathrm{t}, 1 \mathrm{H}, \mathrm{J}=5.6 \mathrm{~Hz}), 1.85(\mathrm{dd}, 3 \mathrm{H}, \mathrm{J}=$ $1.7,6.9 \mathrm{~Hz}), 1.86-1.75(\mathrm{~m}, 1 \mathrm{H}), 1.60-1.20(\mathrm{~m}, 8 \mathrm{H}), 0.87(\mathrm{~d}, 6 \mathrm{H}, \mathrm{J}=6.8 \mathrm{~Hz}) .{ }^{13} \mathrm{C} \mathrm{NMR}$ $\left(100 \mathrm{MHz} ; \mathrm{CDCl}_{3}\right): \delta 166.8,144.5,140.9,138.5,129.5,128.3,128.0,126.3,123.3,78.3$, 63.2, 32.4, 31.7, 31.3, 29.7, 25.6, 18.8, 18.2, 17.8. LRMS (ESI) $\mathrm{m} / \mathrm{z}$ calc'd for $\mathrm{C}_{20} \mathrm{H}_{30} \mathrm{O}_{3} \mathrm{Na}[\mathrm{M}+\mathrm{Na}]^{+}$: 341.4; found: 341.1 . 
But-2-enoic acid 6-(2-formylphenyl)-1-isopropyllhexyl ester (11d):

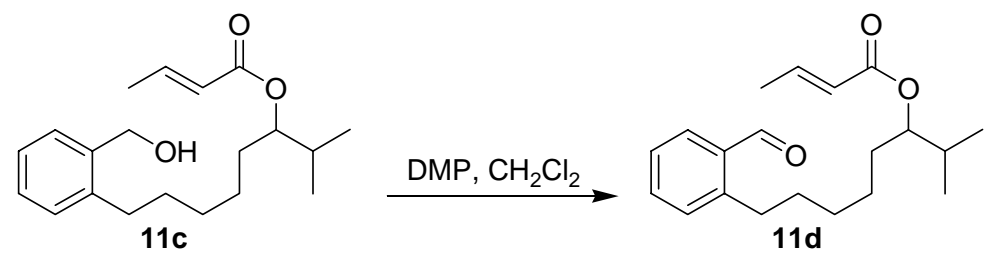

Compound 11d was prepared from 11c by following the procedure for the preparation of $4 \mathbf{a}$ and was purified by flash chromatography (15:1 hexanes:EtOAc) to provide a colorless oil $(0.89 \mathrm{~g}, 90 \%)$.

${ }^{1} \mathrm{H}$ NMR (400 MHz; $\left.\mathrm{CDCl}_{3}\right): \delta 10.25(\mathrm{~s}, 1 \mathrm{H}), 7.80(\mathrm{dd}, 1 \mathrm{H}, \mathrm{J}=1.4,7.7 \mathrm{~Hz}), 7.46$ $(\mathrm{dt}, 1 \mathrm{H}, \mathrm{J}=1.5,7.3 \mathrm{~Hz}), 7.32(\mathrm{dt}, 1 \mathrm{H}, \mathrm{J}=0.9,7.4 \mathrm{~Hz}), 7.22(\mathrm{~d}, 1 \mathrm{H}, \mathrm{J}=7.7 \mathrm{~Hz}), 6.93$ (dq, $1 \mathrm{H}, \mathrm{J}=6.9,15.5 \mathrm{~Hz}), 5.82(\mathrm{dq}, 1 \mathrm{H}, \mathrm{J}=1.7,15.5 \mathrm{~Hz}), 4.75(\mathrm{dd}, 1 \mathrm{H}, \mathrm{J}=5.7,12.2 \mathrm{~Hz})$, 3.01-2.94 (m, 2H), $1.85(\mathrm{dd}, 3 \mathrm{H}, \mathrm{J}=1.7,6.9 \mathrm{~Hz}), 1.84-1.74(\mathrm{~m}, 1 \mathrm{H}), 1.62-1.20(\mathrm{~m}, 8 \mathrm{H})$, $0.86(\mathrm{~d}, 6 \mathrm{H}, \mathrm{J}=6.8 \mathrm{~Hz}) .{ }^{13} \mathrm{C}$ NMR $\left(100 \mathrm{MHz} ; \mathrm{CDCl}_{3}\right): \delta 192.5,166.7,145.9,144.4$, $134.0,133.8,131.7,131.2,126.6,123.3,78.2,32.7,32.5,31.7,31.3,29.7,25.6,18.8$, 18.2, 17.8. IR $\left(\mathrm{cm}^{-1}\right): 2939,2861,1716,1697,1659,1293,1270,1188,752$. HRMS (ESI) $\mathrm{m} / \mathrm{z}$ calc'd for $\mathrm{C}_{20} \mathrm{H}_{28} \mathrm{O}_{3} \mathrm{Na}[\mathrm{M}+\mathrm{Na}]^{+}:$339.1930; found: 339.1932.

\section{General cyclization (intramolecular vinylogous aldol) procedure.}

A solution of the substrate $(0.5 \mathrm{mmol})$ in toluene $(5 \mathrm{~mL})$ was added to a stirred solution of freshly prepared ATPH $(1.1 \mathrm{mmol})$ in toluene $(5 \mathrm{~mL})$ at $-48^{\circ} \mathrm{C}$ (acetonitrile/dry ice bath). After 20 minutes, the ATPH-substrate solution was added dropwise via canula (1 hour) to a stirred solution of LTMP $(1.0 \mathrm{mmol})$ in THF $(5 \mathrm{~mL})$ and toluene $(35 \mathrm{~mL})$ at $-48^{\circ} \mathrm{C}$. After 30 minutes following the addition, saturated $\mathrm{NH}_{4} \mathrm{Cl}$ $(25 \mathrm{~mL})$ was added, and the biphasic mixture was stirred vigorously for 20 minutes while warming to ambient temperature. The mixture was filtered through a small pad of Celite, which was washed with $\mathrm{Et}_{2} \mathrm{O}(50 \mathrm{~mL})$. The organic phase of the filtrate was separated and washed with brine, dried over anhydrous $\mathrm{MgSO}_{4}$, and concentrated under reduced pressure on the rotary evaporator. Purification of the crude product was achieved with flash chromatography (5:1 hexanes:EtOAc then 2:1 hexanes:EtOAc) where 2,6diphenylphenol was also recovered in good yield.

Preparation of ATPH: $\mathrm{Me}_{3} \mathrm{Al}(0.55 \mathrm{~mL}, 1.1 \mathrm{mmol}, 2.0 \mathrm{M}$ solution in hexanes) was added to a stirred solution of 2,6-diphenylphenol $(0.813 \mathrm{~g}, 3.3 \mathrm{mmol})$ in toluene (5 $\mathrm{mL}$ ) at room temperature. After 30 minutes, the ATPH solution was cooled to $-48^{\circ} \mathrm{C}$ and used as described in the cyclization procedure.

Preparation of LTMP: $n B$ Bui $(0.67 \mathrm{~mL}, 1.0 \mathrm{mmol}, 1.50 \mathrm{M}$ solution in hexanes) was added to a stirred solution of TMP $(0.21 \mathrm{~mL}, 1.25 \mathrm{mmol})$ in THF $(5 \mathrm{~mL})$ at $-48^{\circ} \mathrm{C}$. After 30 minutes and immediately before the canulation step in the cyclization procedure, the solution was diluted with cooled toluene $(35 \mathrm{~mL})$. 
12-Hydroxy-5,6,11,12-tetrahydro-7-oxabenzo-(Z)-cyclodecen-8-one (2):

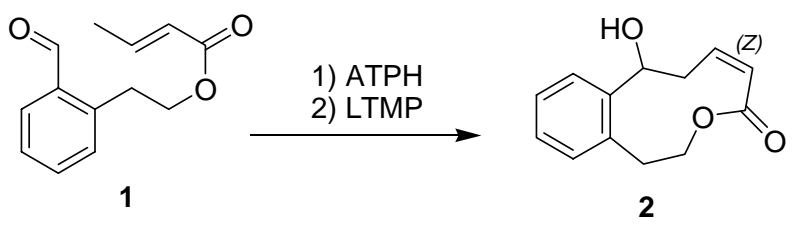

Compound 2 was prepared from 1 in $70 \%$ yield after purification using the general cyclization procedure. We could not detect any diastereomeric material by ${ }^{1} \mathrm{H}$ NMR.

${ }^{1} \mathrm{H}$ NMR $\left(400 \mathrm{MHz} ; \mathrm{CDCl}_{3}\right): \delta 7.54(\mathrm{~d}, 1 \mathrm{H}, \mathrm{J}=7.5 \mathrm{~Hz}), 7.32(\mathrm{dt}, 1 \mathrm{H}, \mathrm{J}=1.3,7.4$ $\mathrm{Hz}), 7.24(\mathrm{dt}, 1 \mathrm{H}, \mathrm{J}=1.5,7.5 \mathrm{~Hz}$ ), 7.17 (dd, $1 \mathrm{H}, \mathrm{J}=1.4,7.6 \mathrm{~Hz}$ ), 6.78 (ddd, $1 \mathrm{H}, \mathrm{J}=6.1$, $8.9,11.1 \mathrm{~Hz}), 5.92(\mathrm{dd}, 1 \mathrm{H}, \mathrm{J}=2.7,11.2 \mathrm{~Hz}), 5.33(\mathrm{dt}, 1 \mathrm{H}, \mathrm{J}=3.6,10.7 \mathrm{~Hz}), 4.84(\mathrm{~d}, 1 \mathrm{H}$, $\mathrm{J}=5.8 \mathrm{~Hz}), 3.84-3.76(\mathrm{~m}, 1 \mathrm{H}), 3.12-3.03(\mathrm{~m}, 1 \mathrm{H}), 3.00(\mathrm{br} \mathrm{d}, 1 \mathrm{H}, \mathrm{J}=12.2 \mathrm{~Hz}), 2.68(\mathrm{br}$ $\mathrm{d}, 1 \mathrm{H}, \mathrm{J}=14.2 \mathrm{~Hz}), 2.53(\mathrm{ddd}, 1 \mathrm{H}, \mathrm{J}=6.5,8.8,14.7 \mathrm{~Hz}), 2.00(\mathrm{br} \mathrm{s}, 1 \mathrm{H}) .{ }^{13} \mathrm{C}$ NMR $(100$ $\left.\mathrm{MHz} ; \mathrm{CDCl}_{3}\right): \delta 166.5,147.0,145.6,135.3,131.0,128.1,125.9,124.1,75.6,66.3,41.1$, 34.0. IR $\left(\mathrm{cm}^{-1}\right): 3412,2959,1714,1274,1185,1027,761$. HRMS (ESI) $\mathrm{m} / \mathrm{z}$ calc'd for $\mathrm{C}_{13} \mathrm{H}_{13} \mathrm{O}_{2}$ [M-OH] $]^{+}: 201.0907$; found: 201.0910 .

\section{2-Hydroxy-5-methyl-5,6,11,12-tetrahydro-7-oxabenzo-(Z)-cyclodecen-8-one (3):}

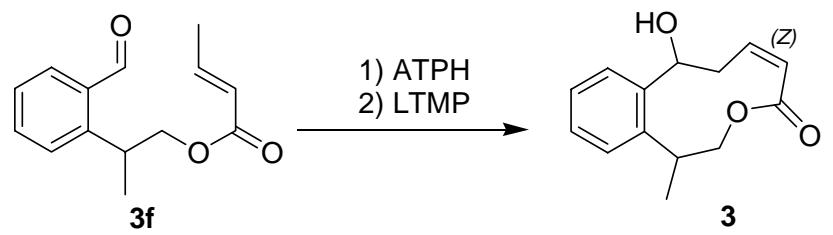

Compound 3 was prepared from $3 \mathbf{f}$ in $80 \%$ yield after purification using the general cyclization procedure. We could not detect any diastereomeric material by ${ }^{1} \mathrm{H}$ NMR.

${ }^{1} \mathrm{H}$ NMR (400 MHz; $\left.\mathrm{CDCl}_{3}\right): \delta$ 7.57-7.53 (m, 1H), 7.35-7.29 (m, 3H), 6.74 (ddd, $1 \mathrm{H}, \mathrm{J}=6.0,8.7,11.4 \mathrm{~Hz}), 5.89(\mathrm{dd}, 1 \mathrm{H}, \mathrm{J}=2.6,11.3 \mathrm{~Hz}), 5.09(\mathrm{dd}, 1 \mathrm{H}, \mathrm{J}=3.4,10.4 \mathrm{~Hz})$, $5.04(\mathrm{dd}, 1 \mathrm{H}, \mathrm{J}=1.0,6.2 \mathrm{~Hz}), 3.57(\mathrm{t}, 1 \mathrm{H}, \mathrm{J}=10.9 \mathrm{~Hz}), 3.36-3.24(\mathrm{~m}, 1 \mathrm{H}), 2.96$ (dddd, $1 \mathrm{H}, \mathrm{J}=1.7,2.7,6.0,14.8 \mathrm{~Hz}$ ), 2.62 (ddd, $1 \mathrm{H}, \mathrm{J}=6.2,8.8,14.9 \mathrm{~Hz}$ ), $1.91(\mathrm{br} \mathrm{s}, 1 \mathrm{H}), 1.36$ $(\mathrm{d}, 3 \mathrm{H}, \mathrm{J}=7.1 \mathrm{~Hz}) .{ }^{13} \mathrm{C}$ NMR $\left(100 \mathrm{MHz} ; \mathrm{CDCl}_{3}\right): \delta 166.5,146.5,145.5,139.1,128.2$, 127.6, 125.7, 125.6, 123.9, 74.1, 71.2, 41.3, 34.0, 15.9. IR $\left(\mathrm{cm}^{-1}\right): 3411,2963,1714$, 1271, 1185, 1030, 758. HRMS (ESI) $\mathrm{m} / \mathrm{z}$ calc'd for $\mathrm{C}_{14} \mathrm{H}_{16} \mathrm{O}_{3} \mathrm{Na}[\mathrm{M}+\mathrm{Na}]^{+}:$255.0991; found: 255.0985 .

\section{2-Hydroxy-6-methyl-5,6,11,12-tetrahydro-7-oxabenzocyclodecen-8-one (4):}<smiles>C/C=C/C(=O)OC(C)Cc1ccccc1C=O</smiles>

$4 e$

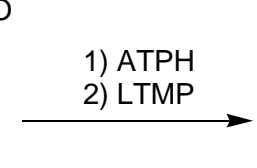

1) ATPH<smiles>COC(C)Cc1ccccc1C(O)CC=CCC(=O)O</smiles>

$4(\mathrm{Z})$<smiles>[CH2+]CC(O)c1ccccc1CC(C)OC(=O)/C=C\C</smiles>

$4(\mathrm{E})$ 
Geometrical isomers $\mathbf{4 ( Z )}$ and $\mathbf{4 ( E )}$ were prepared from $4 \mathbf{e}$ in $77 \%$ yield $[3: 1$ 4(Z):4(E)] after purification using the general cyclization procedure. We could not detect any diastereomeric material by ${ }^{1} \mathrm{H}$ NMR.

4(Z): ${ }^{1} \mathrm{H}$ NMR $\left(500 \mathrm{MHz} ; \mathrm{CDCl}_{3}\right): \delta 7.36(\mathrm{dd}, 1 \mathrm{H}, \mathrm{J}=1.4,7.7 \mathrm{~Hz}), 7.25(\mathrm{dt}, 1 \mathrm{H}$, $\mathrm{J}=1.5,7.4 \mathrm{~Hz}), 7.21(\mathrm{dt}, 1 \mathrm{H}, \mathrm{J}=1.7,7.5 \mathrm{~Hz}), 7.14(\mathrm{dd}, 1 \mathrm{H}, \mathrm{J}=1.7,7.5 \mathrm{~Hz}), 6.07$ (ddd, $1 \mathrm{H}, 5.8,7.0,12.6 \mathrm{~Hz}), 5.56(\mathrm{t}, 1 \mathrm{H}, \mathrm{J}=6.3), 5.53(\mathrm{ddd}, 1 \mathrm{H}, \mathrm{J}=1.1,2.4,12.4 \mathrm{~Hz}), 5.16$ (dddt, 1H, J = 3.0, 6.3, 9.3, $11.4 \mathrm{~Hz}$ ), 3.36 (ddt, 1H, J = 2.4, 5.7, $17.4 \mathrm{~Hz}$ ), 3.02 (dd, 1H, J $=11.4,13.7 \mathrm{~Hz}), 2.84(\mathrm{ddt}, 1 \mathrm{H}, \mathrm{J}=1.2,6.9,17.5 \mathrm{~Hz}), 2.75(\mathrm{dd}, 1 \mathrm{H}, \mathrm{J}=3.1,13.7 \mathrm{~Hz})$, $1.94(\mathrm{~s}, 1 \mathrm{H}), 1.46(\mathrm{~d}, 3 \mathrm{H}, \mathrm{J}=6.4 \mathrm{~Hz}) .{ }^{13} \mathrm{C} \mathrm{NMR}\left(100 \mathrm{MHz} ; \mathrm{CDCl}_{3}\right): \delta 165.7,141.7$, $139.7,135.5,131.0,128.4,127.8,126.1,122.9,73.0,68.7,40.5,39.2,20.9$. IR $\left(\mathrm{cm}^{-1}\right)$ : $3415,2877,2933,1712,1267,1088,1026,760$. HRMS (ESI) $m / z$ calc'd for $\mathrm{C}_{14} \mathrm{H}_{16} \mathrm{O}_{3} \mathrm{Na}$ $[\mathrm{M}+\mathrm{Na}]^{+}:$255.0991; found: 255.0985 .

4(E): ${ }^{1} \mathrm{H}$ NMR $\left(500 \mathrm{MHz} ; \mathrm{CDCl}_{3}\right): \delta 7.76(\mathrm{dd}, 1 \mathrm{H}, \mathrm{J}=1.2,8.0 \mathrm{~Hz}), 7.34(\mathrm{t}, 1 \mathrm{H}$, $\mathrm{J}=7.3 \mathrm{~Hz}), 7.25(\mathrm{dt}, 1 \mathrm{H}, \mathrm{J}=1.4,7.6 \mathrm{~Hz}), 7.10(\mathrm{~d}, 1 \mathrm{H}, \mathrm{J}=7.6 \mathrm{~Hz}), 6.37$ (ddd, 1H, J = 6.8, 9.8, 16.7 Hz), $5.65(\mathrm{~d}, 1 \mathrm{H}, \mathrm{J}=16.8 \mathrm{~Hz}), 4.83(\mathrm{~d}, 1 \mathrm{H}, \mathrm{J}=10.5 \mathrm{~Hz}), 4.26(\mathrm{~m}, 1 \mathrm{H}), 3.03$ $(\mathrm{dd}, 1 \mathrm{H}, \mathrm{J}=1.4,15.3 \mathrm{~Hz}), 2.87(\mathrm{ddd}, 1 \mathrm{H}, \mathrm{J}=1.3,6.7,11.4 \mathrm{~Hz}), 2.83(\mathrm{dd}, 1 \mathrm{H}, \mathrm{J}=5.2$, $15.3 \mathrm{~Hz}), 2.42(\mathrm{dd}, 1 \mathrm{H}, \mathrm{J}=10.3,21.6 \mathrm{~Hz}), 2.09$ (d, 1H, J = 2.4 Hz), $1.68(\mathrm{~d}, 3 \mathrm{H}, \mathrm{J}=6.4$ $\mathrm{Hz}) .{ }^{13} \mathrm{C}$ NMR $\left(100 \mathrm{MHz} ; \mathrm{CDCl}_{3}\right): \delta 169.9,141.1,138.1,137.0,128.3,128.2,128.0$, 126.6, 125.9, 80.3, 69.9, 42.4, 39.9, 25.1. IR $\left(\mathrm{cm}^{-1}\right): 3427,2932,1721,1650,1308$, 1204, 1029, 762, 733. HRMS (ESI) $\mathrm{m} / \mathrm{z}$ calc'd for $\mathrm{C}_{14} \mathrm{H}_{16} \mathrm{O}_{3} \mathrm{Na}[\mathrm{M}+\mathrm{Na}]^{+}$: 255.0991; found: 255.0989 .

\section{2-Hydroxy-6-isopropyl-5,6,11,12-tetrahydro-7-oxabenzocyclodecen-8-one (5):}

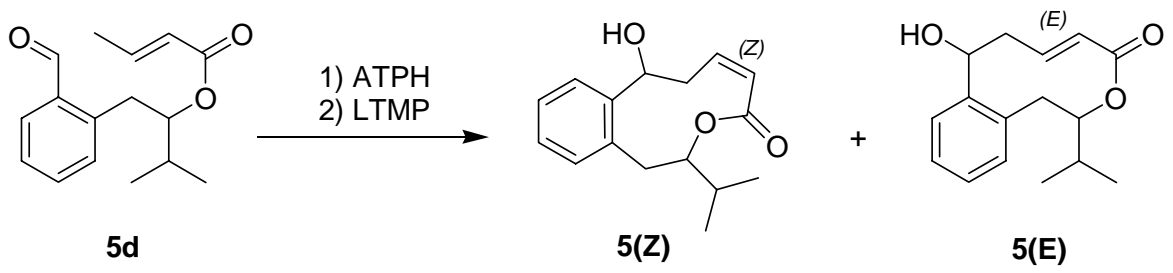

Geometrical isomers $\mathbf{5 ( Z )}$ and $\mathbf{5 ( E )}$ were prepared from $\mathbf{5 d}$ in $82 \%$ yield [1:13 5(Z):5(E)] after purification using the general cyclization procedure. We could not detect any diastereomeric material by ${ }^{1} \mathrm{H}$ NMR.

5(Z): ${ }^{1} \mathrm{H}$ NMR $\left(400 \mathrm{MHz} ; \mathrm{CDCl}_{3}\right): \delta 7.37(\mathrm{dd}, 1 \mathrm{H}, \mathrm{J}=1.7,7.6 \mathrm{~Hz}), 7.25(\mathrm{dt}$, $1 \mathrm{H}, \mathrm{J}=1.6,7.2 \mathrm{~Hz}), 7.21(\mathrm{dt}, 1 \mathrm{H}, \mathrm{J}=1.6,7.1 \mathrm{~Hz}), 7.15(\mathrm{dd}, 1 \mathrm{H}, \mathrm{J}=1.8,7.3 \mathrm{~Hz}), 6.09$ (ddd, $1 \mathrm{H}, \mathrm{J}=6.0,7.1,12.7 \mathrm{~Hz}), 5.58-5.52(\mathrm{~m}, 1 \mathrm{H}), 5.53(\mathrm{ddd}, 1 \mathrm{H}, \mathrm{J}=1.0,2.6,12.5 \mathrm{~Hz}$ ), 4.86 (ddd, 1H, J = 3.1, 6.3, $11.6 \mathrm{~Hz}), 3.49$ (ddt, $1 \mathrm{H}, \mathrm{J}=2.5,5.8,17.2 \mathrm{~Hz}), 3.03$ (dd, 1H, J $=11.7,13.4 \mathrm{~Hz}), 2.92-2.83(\mathrm{~m}, 1 \mathrm{H}), 2.76(\mathrm{dd}, 1 \mathrm{H}, \mathrm{J}=3.1,13.5 \mathrm{~Hz}), 2.00(\mathrm{o}, 1 \mathrm{H}, \mathrm{J}=6.7$ $\mathrm{Hz}), 1.65(\mathrm{~d}, 1 \mathrm{H}, \mathrm{J}=3.0 \mathrm{~Hz}), 1.06(\mathrm{~d}, 3 \mathrm{H}, \mathrm{J}=6.9 \mathrm{~Hz}), 1.02(\mathrm{~d}, 3 \mathrm{H}, \mathrm{J}=6.9 \mathrm{~Hz}) .{ }^{13} \mathrm{C}$ NMR $\left(100 \mathrm{MHz} ; \mathrm{CDCl}_{3}\right): \delta 165.9,141.5,139.8,135.8,131.1,128.5,127.8,126.2$, 123.0, 81.4, 68.8, 38.7, 35.6, 32.6, 19.1, 18.6. IR ( $\left.\mathrm{cm}^{-1}\right): 3413,2964,1714,1266,996$, 758. HRMS (ESI) $\mathrm{m} / \mathrm{z}$ calc'd for $\mathrm{C}_{16} \mathrm{H}_{20} \mathrm{O}_{3} \mathrm{~K}[\mathrm{M}+\mathrm{K}]^{+}$: 299.1044; found: 299.1053.

5(E): ${ }^{1} \mathrm{H}$ NMR $\left(400 \mathrm{MHz} ; \mathrm{CDCl}_{3}\right): \delta 7.74(\mathrm{dd}, 1 \mathrm{H}, \mathrm{J}=1.5,8.0 \mathrm{~Hz}), 7.33(\mathrm{dt}$, $1 \mathrm{H}, \mathrm{J}=1.3,7.9 \mathrm{~Hz}), 7.25(\mathrm{dt}, 1 \mathrm{H}, \mathrm{J}=1.6,7.7 \mathrm{~Hz}), 7.00(\mathrm{~d}, 1 \mathrm{H}, \mathrm{J}=7.7 \mathrm{~Hz}), 6.41$ (ddd, $1 \mathrm{H}, \mathrm{J}=6.7,9.9,16.6 \mathrm{~Hz}), 5.55(\mathrm{~d}, 1 \mathrm{H}, \mathrm{J}=16.7 \mathrm{~Hz}), 4.84(\mathrm{~d}, 1 \mathrm{H}, \mathrm{J}=10.5 \mathrm{~Hz}), 4.07(\mathrm{dt}$, $1 \mathrm{H}, \mathrm{J}=2.5,4.8 \mathrm{~Hz}$ ), 2.95 (dd, $1 \mathrm{H}, \mathrm{J}=4.5,15.6 \mathrm{~Hz}$ ), 2.86 (dddd, 1H, J = 0.6, 1.8, 6.7, $11.6 \mathrm{~Hz}), 2.81(\mathrm{dd}, 1 \mathrm{H}, \mathrm{J}=2.5,15.6 \mathrm{~Hz}), 2.39(\mathrm{dq}, 1 \mathrm{H}, \mathrm{J}=0.6,10.6 \mathrm{~Hz}), 2.17$ (dh, 1H, J 
$=2.5,6.8 \mathrm{~Hz}), 2.07(\mathrm{~d}, 1 \mathrm{H}, \mathrm{J}=2.5 \mathrm{~Hz}), 1.12(\mathrm{~d}, 6 \mathrm{H}, \mathrm{J}=6.8 \mathrm{~Hz}) .{ }^{13} \mathrm{C} \mathrm{NMR}(100 \mathrm{MHz}$; $\left.\mathrm{CDCl}_{3}\right): \delta 170.6,141.2,138.2,138.0,128.3,128.2,127.9,126.4,126.1,88.2,69.7,42.4$, 36.0, 35.8, 20.4, 15.0. IR ( $\left.\mathrm{cm}^{-1}\right): 3429,2966,2933,1713,1650,1206,1034,1015,762$, 735. HRMS (ESI) $\mathrm{m} / \mathrm{z}$ calc' $d$ for $\mathrm{C}_{16} \mathrm{H}_{20} \mathrm{O}_{3} \mathrm{~K}$ [M+K] $]^{+}$: 299.1044; found: 299.1050.

\section{4-Hydroxy-5,6,7,8,13,14-hexahydro-9-oxabenzo-(E)-cyclododecen-10-one (6):}

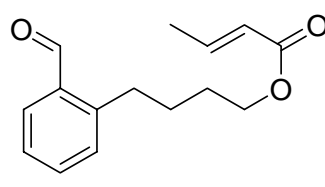

$6 e$

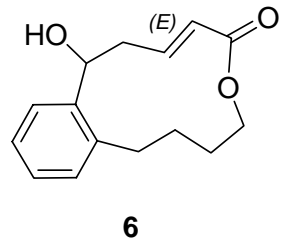

6

Compound 6 was prepared from $\mathbf{6 e}$ in $81 \%$ yield after purification using the general cyclization procedure. The macrodiolide was also isolated in the cyclization $(7 \%$ yield). We could not detect any diastereomeric material by ${ }^{1} \mathrm{H}$ NMR.

${ }^{1} \mathrm{H}$ NMR $\left(400 \mathrm{MHz} ; \mathrm{CDCl}_{3}\right): \delta 7.58($ br s, $1 \mathrm{H}), 7.28-7.18(\mathrm{~m}, 2 \mathrm{H}), 7.16-7.09(\mathrm{~m}$, 1H), 6.68 (br s, 1H), 5.78 (d, 1H, J = 15.9 Hz), 4.98 (br d, 1H, J = 9.5 Hz), 4.45-4.34 (br m, 1H), 4.26-4.13 (br m, 1H), 2.96-2.84 (br m, 1H), 2.68-2.44 (br m, 3H), 2.14-1.96 (m, $2 \mathrm{H}), 1.86-1.74$ (br m, 1H), 1.64-1.50 (br m, 2H). ${ }^{1} \mathrm{H} \mathrm{NMR}\left(\mathrm{T}=59^{\circ} \mathrm{C}, 400 \mathrm{MHz} ; \mathrm{CDCl}_{3}\right)$ : $\delta 7.55$ (br d, $1 \mathrm{H}, \mathrm{J}=7.0 \mathrm{~Hz}), 7.28-7.18(\mathrm{~m}, 2 \mathrm{H}), 7.15-7.10(\mathrm{~m}, 1 \mathrm{H}), 6.69(\mathrm{dt}, 1 \mathrm{H}, \mathrm{J}=8.2$, $16.1 \mathrm{~Hz}), 5.77(\mathrm{~d}, 1 \mathrm{H}, \mathrm{J}=15.8 \mathrm{~Hz}), 5.00(\mathrm{br} d t, 1 \mathrm{H}, \mathrm{J}=3.1,10.2 \mathrm{~Hz}), 4.38$ (ddd, 1H, J = $3.8,6.0,9.8 \mathrm{~Hz}$ ), 4.24 (ddd, $1 \mathrm{H}, \mathrm{J}=3.7,8.6,11.5 \mathrm{~Hz}$ ), 2.94-2.86 (br m, 1H), 2.70-2.62 $(\mathrm{m}, 2 \mathrm{H}), 2.54(\mathrm{dt}, 1 \mathrm{H}, \mathrm{J}=7.5,10.8 \mathrm{~Hz}), 2.10-1.97$ (br m, 1H), 1.84 (d, 1H, J = 2.8 Hz), $1.85-1.74(\mathrm{~m}, 1 \mathrm{H}), 1.70-1.54(\mathrm{~m}, 2 \mathrm{H}) .{ }^{13} \mathrm{C} \mathrm{NMR}\left(100 \mathrm{MHz} ; \mathrm{CDCl}_{3}\right): \delta 167.5,147.0$, $141.4,138.9,128.0,126.9,126.0,124.8,70.8,66.8,43.1,30.6,27.7,25.7 . \quad$ IR $\left(\mathrm{cm}^{-1}\right)$ : 3429, 2932, 1712, 1644, 1246, 1034, 763. HRMS (ESI) $\mathrm{m} / \mathrm{z}$ calc'd for $\mathrm{C}_{15} \mathrm{H}_{18} \mathrm{O}_{3} \mathrm{Na}$ $[\mathrm{M}+\mathrm{Na}]^{+}:$269.1148; found: 269.1145 .

14-Hydroxy-8-methyl-5,6,7,8,13,14-hexahydro-9-oxabenzo-(E)-cyclododecen-10-one (7):
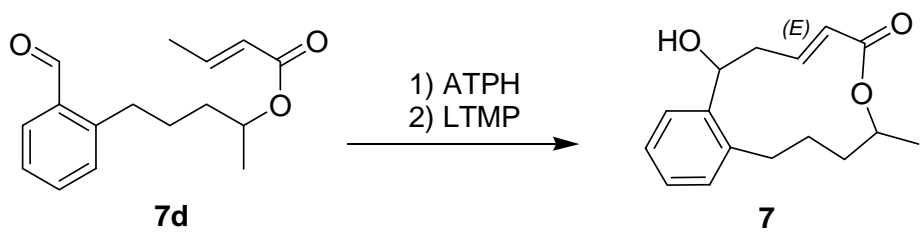

Compound 7 was prepared from $\mathbf{7 d}$ in $84 \%$ yield after purification using the general cyclization procedure. We could not detect any diastereomeric material by ${ }^{1} \mathrm{H}$ NMR.

${ }^{1} \mathrm{H}$ NMR (500 MHz; $\left.\mathrm{CDCl}_{3}\right): \delta 7.52($ br s, $1 \mathrm{H}), 7.23-7.14(\mathrm{~m}, 3 \mathrm{H}), 7.05(\mathrm{br} \mathrm{d}, 1 \mathrm{H}$, $\mathrm{J}=6.4 \mathrm{~Hz}), 6.80(\mathrm{ddd}, 1 \mathrm{H}, \mathrm{J}=5.5,10.9,16.0 \mathrm{~Hz}), 5.47(\mathrm{br} \mathrm{d}, 1 \mathrm{H}, \mathrm{J}=13.6 \mathrm{~Hz}), 5.08$ (dd, $1 \mathrm{H}, \mathrm{J}=3.4,10.7 \mathrm{~Hz}$ ), 5.04 (br s, 1H), 2.96 (br s, 1H), 2.70-2.52 (m, 2H), 2.49-2.36 (br m, 1H), 2.12 (br s, 1H), 2.00-1.80 (br m, 2H), 1.46 (br s, 1H), 1.29 (d, 3H, J = 6.4 Hz), 1.16 (br s, $1 \mathrm{H}) .{ }^{1} \mathrm{H} \mathrm{NMR}\left(\mathrm{T}=59^{\circ} \mathrm{C}, 500 \mathrm{MHz} ; \mathrm{CDCl}_{3}\right): \delta 7.50($ br d, $1 \mathrm{H}, \mathrm{J}=7.4 \mathrm{~Hz}), 7.21$ (br t, 1H, J = 7.3 Hz), 7.17 (dt, 1H, J = 1.6, 7.3 Hz), 7.07 (br d, 1H, J = 7.6 Hz), 6.77 (ddd, $1 \mathrm{H}, \mathrm{J}=5.8,10.6,16.1 \mathrm{~Hz}$ ), 5.54 (br d, 1H, J = 15.8 Hz), 5.13-5.08 (br m, 1H), 5.04 
(ddq, 1H, J = 3.2, 6.4, 9.5 Hz), 2.99-2.91 (m, 1H), 2.74-2.57 (m, 2H), 2.46 (q, 1H, J = $10.7 \mathrm{~Hz}$ ), 2.16-2.03 (br m, 1H), 1.86 (ddt, $1 \mathrm{H}, \mathrm{J}=2.9,9.1,14.9 \mathrm{~Hz}), 1.82$ (d, 1H, J = 2.4 $\mathrm{Hz}), 1.53-1.42(\mathrm{~m}, 1 \mathrm{H}), 1.30(\mathrm{~d}, 3 \mathrm{H}, \mathrm{J}=6.4 \mathrm{~Hz}), 1.34-1.26(\mathrm{~m}, 1 \mathrm{H}) .{ }^{13} \mathrm{C} \mathrm{NMR}(100$ $\left.\mathrm{MHz} ; \mathrm{CDCl}_{3}\right): \delta 167.6,144.5,140.9,139.5,127.9,126.7,125.8,125.0,74.4,70.5,43.1$, 35.3, 31.2, 30.0, 25.0, 20.6, 14.3. IR ( $\left.\mathrm{cm}^{-1}\right): 3432,2931,1711,1645,1251,1034,1001$, 761. HRMS (ESI) $\mathrm{m} / \mathrm{z}$ calc'd for $\mathrm{C}_{16} \mathrm{H}_{20} \mathrm{O}_{3} \mathrm{~K}[\mathrm{M}+\mathrm{K}]^{+}$: 299.1044; found: 299.1048.

\section{4-Hydroxy-8-isopropyl-5,6,7,8,13,14-hexahydro-9-oxabenzo-(E)-cyclododecen-10-} one (8):
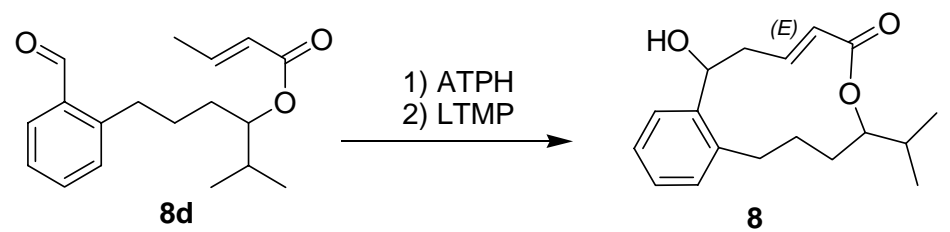

Compound 8 was prepared from 8d in 83\% yield after purification using the general cyclization procedure. We could not detect any diastereomeric material by ${ }^{1} \mathrm{H}$ NMR.

${ }^{1} \mathrm{H}$ NMR $\left(400 \mathrm{MHz} ; \mathrm{CDCl}_{3}\right): \delta 7.52(\mathrm{br} \mathrm{d}, 1 \mathrm{H}, \mathrm{J}=6.2 \mathrm{~Hz}), 7.23-7.13(\mathrm{~m}, 2 \mathrm{H})$, 7.05 (br d, 1H, J = 7.6 Hz), 6.82 (ddd, 1H, J = 5.4, 10.9, $16.0 \mathrm{~Hz}$ ), 5.49 (br d, 1H, J = 15.7 $\mathrm{Hz}$ ), 5.08 (br d, 1H, J = 9.4 Hz), 4.63 (ddd, 1H, J = 3.0, 6.7, $10.0 \mathrm{~Hz}$ ), 3.00-2.91 (br m, $1 \mathrm{H}$ ), 2.64-2.52 (br m, 2H), 2.42 (br q, $1 \mathrm{H}, \mathrm{J}=10.9 \mathrm{~Hz}$ ), 2.20-2.04 (br m, 1H), 2.03-1.98 (br d, $1 \mathrm{H}, \mathrm{J}=1.8 \mathrm{~Hz}$ ), 1.92-1.74 (m, 2H), 1.54-1.42 (br m, 1H), 1.08-0.96 (br m, 1H), $0.94(\mathrm{~d}, 3 \mathrm{H}, \mathrm{J}=2.8 \mathrm{~Hz}), 0.92(\mathrm{~d}, 3 \mathrm{H}, \mathrm{J}=2.9 \mathrm{~Hz}) .{ }^{1} \mathrm{H} \mathrm{NMR}\left(\mathrm{T}=59^{\circ} \mathrm{C}, 500 \mathrm{MHz}\right.$; $\left.\mathrm{CDCl}_{3}\right): \delta 7.52(\mathrm{~d}, 1 \mathrm{H}, \mathrm{J}=7.6 \mathrm{~Hz}), 7.21(\mathrm{dt}, 1 \mathrm{H}, \mathrm{J}=1.3,7.8 \mathrm{~Hz}), 7.16(\mathrm{dt}, 1 \mathrm{H}, \mathrm{J}=1.5$, $7.2 \mathrm{~Hz}), 7.06(\mathrm{~d}, 1 \mathrm{H}, \mathrm{J}=7.7 \mathrm{~Hz}), 6.78(\mathrm{ddd}, 1 \mathrm{H}, \mathrm{J}=5.6,10.7,16.1 \mathrm{~Hz}), 5.55(\mathrm{~d}, 1 \mathrm{H}, \mathrm{J}=$ $15.9 \mathrm{~Hz}), 5.11(\mathrm{dt}, 1 \mathrm{H}, \mathrm{J}=2.8,10.3 \mathrm{~Hz}), 4.64(\mathrm{ddd}, 1 \mathrm{H}, \mathrm{J}=3.0,6.6,10.0 \mathrm{~Hz}), 3.00-2.92$ $(\mathrm{m}, 1 \mathrm{H}), 2.69-2.55(\mathrm{~m}, 2 \mathrm{H}), 2.45(\mathrm{q}, 1 \mathrm{H}, \mathrm{J}=10.8 \mathrm{~Hz}), 2.17-2.04(\mathrm{~m}, 1 \mathrm{H}), 1.91-1.76(\mathrm{~m}$, $3 \mathrm{H}), 1.56-1.45$ (br m, 1H), 1.16-1.06 (br m, 1H), 0.96 (d, 3H, J = 3.3 Hz), 0.94 (d, 3H, J = $3.3 \mathrm{~Hz}) .{ }^{13} \mathrm{C}$ NMR $\left(100 \mathrm{MHz} ; \mathrm{CDCl}_{3}\right): \delta 167.8,144.4,140.8,139.7,127.8,126.7$, $125.7,124.9,83.1,70.5,43.1,32.3,31.5,31.0,25.4,19.1,18.6$. IR $\left(\mathrm{cm}^{-1}\right): 3431,2962$, $2875,1712,1645,1247,1034,1003,760$. HRMS (ESI) $\mathrm{m} / \mathrm{z}$ calc'd for $\mathrm{C}_{18} \mathrm{H}_{24} \mathrm{O}_{3} \mathrm{Na}$ $[\mathrm{M}+\mathrm{Na}]^{+}:$311.1617; found: 311.1606 .

\section{5-Hydroxyl-5,6,11,12,13,14,15,16-octohydro-10-oxabenzo-(E)-tetradecen-9-one (9):}

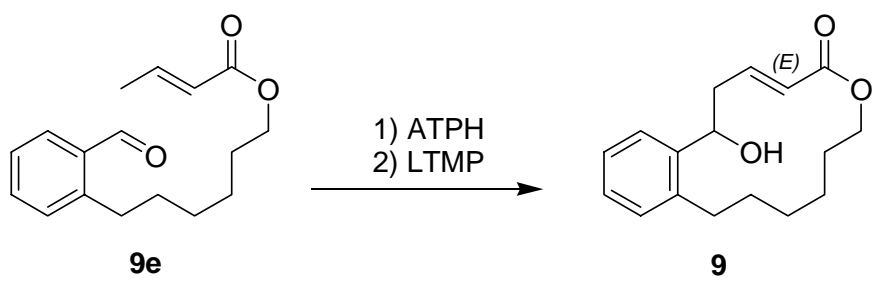

Compound 9 was prepared from 9e in 90\% yield after purification using the general cyclization procedure. We could not detect any diastereomeric material by ${ }^{1} \mathrm{H}$ NMR.

${ }^{1} \mathrm{H}$ NMR $\left(400 \mathrm{MHz} ; \mathrm{CDCl}_{3}\right): \delta$ 7.57-7.52 (m, 1H), 7.27-7.14 (m, 3H), 6.54 (ddd, $1 \mathrm{H}, \mathrm{J}=6.7,8.9,15.7 \mathrm{~Hz}), 5.73(\mathrm{dt}, 1 \mathrm{H}, \mathrm{J}=1.3,15.7 \mathrm{~Hz}), 5.10(\mathrm{dd}, 1 \mathrm{H}, \mathrm{J}=3.6,11.1 \mathrm{~Hz})$, 
4.19 (ddd, 1H, J = 2.3, 5.5, $10.9 \mathrm{~Hz}$ ), 4.12 (ddd, 1H, J = 1.9, 9.6, $11.1 \mathrm{~Hz}$ ), 2.88 (dddd, $1 \mathrm{H}, \mathrm{J}=1.1,3.6,8.9,12.8 \mathrm{~Hz}), 2.65-2.54(\mathrm{~m}, 2 \mathrm{H}), 2.49$ (ddd, $1 \mathrm{H}, \mathrm{J}=4.9,9.4,14.2 \mathrm{~Hz})$, 1.97 (br s, $1 \mathrm{H}), 1.78-1.21(\mathrm{~m}, 8 \mathrm{H}) .{ }^{13} \mathrm{C} \mathrm{NMR}\left(100 \mathrm{MHz} ; \mathrm{CDCl}_{3}\right): \delta 165.8,144.3,140.9$, $140.0,129.0,128.0,126.8,126.1,124.6,69.3,66.1,43.2,30.9,30.0,28.6,28.0,25.7$. IR $\left(\mathrm{cm}^{-1}\right): 3427,2930,2856,1716,1643,1266,1016,984,763$. HRMS (ESI) $\mathrm{m} / \mathrm{z}$ calc'd for $\mathrm{C}_{17} \mathrm{H}_{22} \mathrm{O}_{3} \mathrm{Na}[\mathrm{M}+\mathrm{Na}]^{+}$: 297.1461; found: 297.1468 .

\section{5-Hydroxyl-11-methyl-5,6,11,12,13,14,15,16-octohydro-10-oxabenzo-(E)-tetradecen- 9-one (10):}

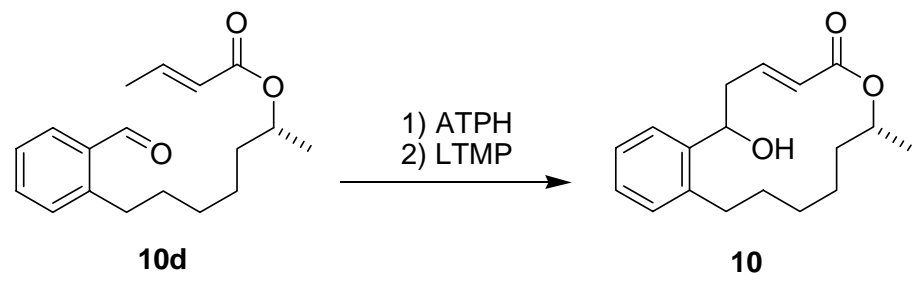

Compound 10 was prepared from 10d in $89 \%$ yield after purification using the general cyclization procedure. We could not detect any diastereomeric material by ${ }^{1} \mathrm{H}$ NMR.

${ }^{1} \mathrm{H}$ NMR $\left(400 \mathrm{MHz} ; \mathrm{CDCl}_{3}\right): \delta 7.47(\mathrm{dd}, 1 \mathrm{H}, \mathrm{J}=1.6,7.3 \mathrm{~Hz}), 7.24-7.15(\mathrm{~m}, 2 \mathrm{H})$, $7.10(\mathrm{dd}, 1 \mathrm{H}, \mathrm{J}=1.7,7.3 \mathrm{~Hz}), 6.66(\mathrm{ddd}, 1 \mathrm{H}, \mathrm{J}=5.2,10.6,15.8 \mathrm{~Hz}), 5.51(\mathrm{dd}, 1 \mathrm{H}, \mathrm{J}=$ $1.1,15.7 \mathrm{~Hz}), 5.23(\mathrm{dt}, 1 \mathrm{H}, \mathrm{J}=3.0,10.8 \mathrm{~Hz}), 4.97(\mathrm{dp}, 1 \mathrm{H}, \mathrm{J}=2.4,6.4 \mathrm{~Hz}), 2.98-2.90(\mathrm{~m}$, $1 \mathrm{H}), 2.64-2.54(\mathrm{~m}, 3 \mathrm{H}), 1.83(\mathrm{~d}, 1 \mathrm{H}, \mathrm{J}=2.7 \mathrm{~Hz}), 1.76-1.58(\mathrm{~m}, 3 \mathrm{H}), 1.53-1.24(\mathrm{~m}, 5 \mathrm{H})$, $1.23(\mathrm{~d}, 3 \mathrm{H}, \mathrm{J}=6.6 \mathrm{~Hz}) .{ }^{13} \mathrm{C} \mathrm{NMR}\left(100 \mathrm{MHz} ; \mathrm{CDCl}_{3}\right): \delta 165.5,143.5,140.6,139.9$, 129.1, 128.0, 126.8, 126.1, 125.2, 71.9, 42.6, 32.0, 30.9, 30.4, 28.8, 24.6, 19.1. IR ( $\left.\mathrm{cm}^{-1}\right)$ : $3419,2935,2859,1712,1645,1270,1034,982,760$. $[\alpha]_{\mathrm{D}}+13.9\left(\right.$ c $\left.1.14,26^{\circ} \mathrm{C}, \mathrm{CH}_{2} \mathrm{Cl}_{2}\right)$. HRMS (ESI) $\mathrm{m} / \mathrm{z}$ calc'd for $\mathrm{C}_{18} \mathrm{H}_{24} \mathrm{O}_{3} \mathrm{Na}[\mathrm{M}+\mathrm{Na}]^{+}: 311.1617$; found: 311.1619 .

\section{5-Hydroxyl-11-isopropyl-5,6,11,12,13,14,15,16-octohydro-10-oxabenzo-(E)- tetradecen-9-one (11):}

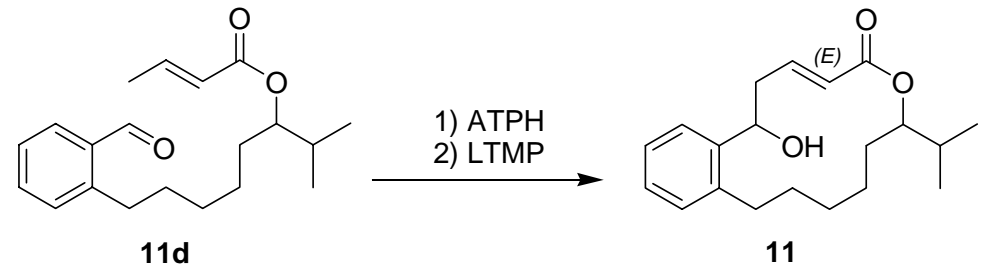

Compound 11 was prepared from 11d in 88\% yield after purification using the general cyclization procedure. We could not detect any diastereomeric material by ${ }^{1} \mathrm{H}$ NMR.

${ }^{1} \mathrm{H}$ NMR $\left(400 \mathrm{MHz} ; \mathrm{CDCl}_{3}\right): \delta 7.47(\mathrm{dd}, 1 \mathrm{H}, \mathrm{J}=1.4,7.5 \mathrm{~Hz}), 7.21(\mathrm{dt}, 1 \mathrm{H}, \mathrm{J}=$ 1.5, 7.2 Hz), $7.16(\mathrm{dt}, 1 \mathrm{H}, \mathrm{J}=1.7,7.2 \mathrm{~Hz}), 7.08(\mathrm{dd}, 1 \mathrm{H}, \mathrm{J}=1.4,7.3 \mathrm{~Hz}), 6.69$ (ddd, $1 \mathrm{H}, \mathrm{J}$ $=5.1,10.9,15.8 \mathrm{~Hz}), 5.50(\mathrm{dd}, 1 \mathrm{H}, \mathrm{J}=1.4,15.7 \mathrm{~Hz}), 5.20(\mathrm{dd}, 1 \mathrm{H}, \mathrm{J}=3.8,10.8 \mathrm{~Hz})$, 4.61-4.54 (m, 1H), 2.97-2.90 (m, 1H), 2.62-2.51 (m, 3H), 2.00-1.86 (m, 2H), 1.69-1.51 $(\mathrm{m}, 4 \mathrm{H}), 1.43-1.20(\mathrm{~m}, 4 \mathrm{H}), 0.88(\mathrm{~d}, 3 \mathrm{H}, \mathrm{J}=4.4 \mathrm{~Hz}), 0.86(\mathrm{~d}, 3 \mathrm{H}, \mathrm{J}=4.4 \mathrm{~Hz}) .{ }^{13} \mathrm{C} \mathrm{NMR}$ $\left(100 \mathrm{MHz} ; \mathrm{CDCl}_{3}\right): \delta 165.7,143.2,140.6,139.9,128.9,128.0,126.7,126.0,125.3,80.3$, 
$42.8,30.8,30.1,30.0,28.8,27.3,24.2,19.4,18.7$. IR $\left(\mathrm{cm}^{-1}\right): 3416,2934,2860,1713$, 1646, 1267, 1035, 988, 760. HRMS (ESI) $\mathrm{m} / \mathrm{z}$ calc'd for $\mathrm{C}_{20} \mathrm{H}_{28} \mathrm{O}_{3} \mathrm{Na}[\mathrm{M}+\mathrm{Na}]^{+}$: 339.1930; found: 339.1934 .

\section{Hydrogenation of $4(Z) / 4(E)$ and $5(Z) / 5(E)$.}

\section{2-Hydroxy-6-methyl-5,6,9,10,11,12-hexahydro-7-oxa-benzocyclodecen-8-one (12):}<smiles>COC(C)Cc1ccccc1C(O)CC=CC(=O)O</smiles>

$4(\mathrm{Z})$<smiles>CC/C=C\C(=O)OC(C)Cc1ccccc1C(O)C[18O]</smiles>

$4(E)$

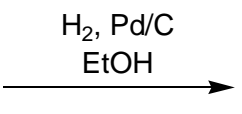

A mixture of lactone $4(Z)$ and palladium on carbon $(5 \mathrm{~mol} \%)$ in absolute EtOH was vigorously stirred under a hydrogen atmosphere overnight at room temperature. The reaction mixture was filtered through a pad of silica gel, which was washed with EtOAc, and then the resulting filtrate was concentrated under reduced pressure on the rotary evaporator. Compound $\mathbf{1 2}$ was produced quantitatively and did not need further purification. Treating lactone $\mathbf{4}(\boldsymbol{E})$ to the same conditions also provided compound 12 quantitatively.

${ }^{1} \mathrm{H}$ NMR $\left(400 \mathrm{MHz} ; \mathrm{CDCl}_{3}\right): \delta 7.41(\mathrm{dd}, 1 \mathrm{H}, \mathrm{J}=1.6,7.7 \mathrm{~Hz}), 7.28(\mathrm{dt}, 1 \mathrm{H}, \mathrm{J}=$ 1.5, $7.3 \mathrm{~Hz}), 7.24(\mathrm{dt}, 1 \mathrm{H}, \mathrm{J}=1.6,7.2 \mathrm{~Hz}), 7.16(\mathrm{dd}, 1 \mathrm{H}, \mathrm{J}=1.7,7.4 \mathrm{~Hz}), 5.39(\mathrm{dd}, 1 \mathrm{H}, \mathrm{J}$ $=5.0,9.9 \mathrm{~Hz}), 5.10(\mathrm{ddq}, 1 \mathrm{H}, \mathrm{J}=2.3,6.4,11.4 \mathrm{~Hz}), 3.12(\mathrm{dd}, 1 \mathrm{H}, \mathrm{J}=11.4,13.9 \mathrm{~Hz})$, $2.68(\mathrm{dd}, 1 \mathrm{H}, \mathrm{J}=2.3,14.0 \mathrm{~Hz}), 2.30-2.05(\mathrm{~m}, 4 \mathrm{H}), 1.99-1.88(\mathrm{~m}, 1 \mathrm{H}), 1.54($ br s, $1 \mathrm{H})$, $1.41(\mathrm{~d}, 3 \mathrm{H}, \mathrm{J}=6.4 \mathrm{~Hz}) .{ }^{13} \mathrm{C}$ NMR $\left(100 \mathrm{MHz} ; \mathrm{CDCl}_{3}\right): \delta 173.0,141.4,136.6,131.3$, $128.8,128.0,126.7,72.2,68.5,40.7,38.2,35.3,23.2,20.9$. HRMS (ESI) $\mathrm{m} / \mathrm{z}$ calc'd for $\mathrm{C}_{14} \mathrm{H}_{18} \mathrm{O}_{3} \mathrm{Na}[\mathrm{M}+\mathrm{Na}]^{+}:$257.1148; found: 257.1145 .

\section{2-Hydroxy-6-isopropyl-5,6,9,10,11,12-hexahydro-7-oxa-benzocyclodecen-8-one} (13):

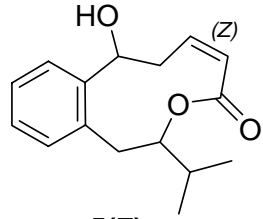

$5(Z)$

(or)

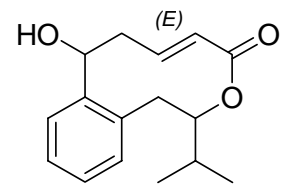

$5(E)$<smiles>COC(Cc1ccccc1C(O)CCCC(=O)O)C(C)C</smiles>

13

Compound 13 was prepared quantitatively from either $5(Z)$ or $\mathbf{5 ( E )}$ by following the procedure for the preparation of 12.

${ }^{1} \mathrm{H} \mathrm{NMR}\left(400 \mathrm{MHz} ; \mathrm{CDCl}_{3}\right): \delta 7.39(\mathrm{dd}, 1 \mathrm{H}, \mathrm{J}=1.7,7.6 \mathrm{~Hz}), 7.27(\mathrm{dt}, 1 \mathrm{H}, \mathrm{J}=$ 1.6, $7.3 \mathrm{~Hz}), 7.22(\mathrm{dt}, 1 \mathrm{H}, \mathrm{J}=1.6,7.2 \mathrm{~Hz}), 7.14(\mathrm{dd}, 1 \mathrm{H}, \mathrm{J}=1.7,7.4 \mathrm{~Hz}), 5.31(\mathrm{dd}, 1 \mathrm{H}, \mathrm{J}$ $=4.8,10.1 \mathrm{~Hz}), 4.77(\mathrm{ddd}, 1 \mathrm{H}, \mathrm{J}=2.3,6.0,11.5 \mathrm{~Hz}), 3.08(\mathrm{dd}, 1 \mathrm{H}, \mathrm{J}=11.6,13.7 \mathrm{~Hz})$, $2.67(\mathrm{dd}, 1 \mathrm{H}, \mathrm{J}=2.2,13.7 \mathrm{~Hz}), 2.29-1.88(\mathrm{~m}, 6 \mathrm{H}), 1.68(\mathrm{~s}, 1 \mathrm{H}), 1.49-1.38(\mathrm{~m}, 1 \mathrm{H}), 1.03$ $(\mathrm{d}, 3 \mathrm{H}, \mathrm{J}=6.8 \mathrm{~Hz}), 1.00(\mathrm{~d}, 3 \mathrm{H}, \mathrm{J}=6.8 \mathrm{~Hz}) .{ }^{13} \mathrm{C} \mathrm{NMR}\left(100 \mathrm{MHz} ; \mathrm{CDCl}_{3}\right): \delta 172.9$, $141.5,136.8,131.4,128.7,127.8,127.0,80.2$, 68.5, 38.2, 35.6, 35.0, 32.3, 23.1, 19.1, 18.3. HRMS (ESI) $\mathrm{m} / \mathrm{z}$ calc'd for $\mathrm{C}_{16} \mathrm{H}_{22} \mathrm{O}_{3} \mathrm{Na}[\mathrm{M}+\mathrm{Na}]^{+}: 285.1461$; found: 285.1471 . 


\section{Determination of Stereochemisty for Lactones 3, 4, 7, and 10.}

The relative stereochemistry for lactones 3, 4, and 7 was determined by X-ray crystallography of their 3,5-dinitrobenzoate derivatives. The stereochemistry for the related lactones $\mathbf{5}$ and $\mathbf{8}$ were assigned by analogy.

The absolute stereochemistry for lactone $\mathbf{1 0}$ was determined by correlation with a related compound of known stereochemistry. The stereochemistry for the related lactone 11 was assigned by analogy.

\section{Derivatization of 3 to the 3,5-dinitrobenzoate 14 (X-ray structure).}

\section{(Z)-2,4,7,8-tetrahydro-1-methyl-4-oxo-1H-benzo[d]oxecin-8-yl 3,5-dinitrobenzoate} (14):

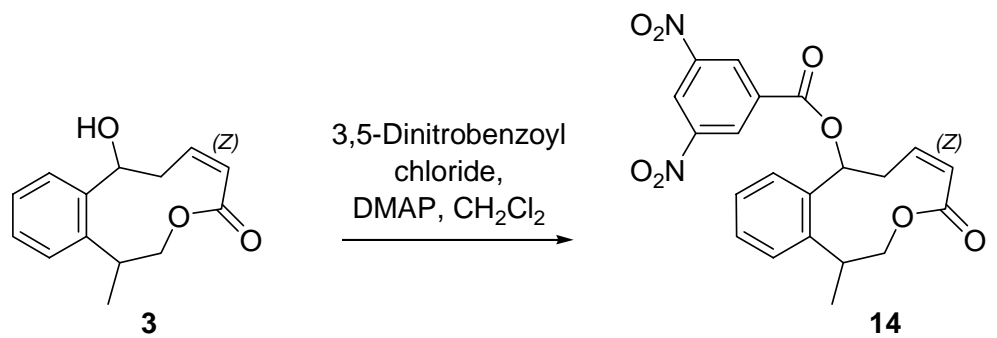

3,5-Dintrobenzoyl chloride (104 mg, $0.45 \mathrm{mmol}$ ) and DMAP (53 mg, $0.45 \mathrm{mmol}$ ) was added sequentially to a solution of lactone $3(70 \mathrm{mg}, 0.3 \mathrm{mmol})$ in $\mathrm{CH}_{2} \mathrm{Cl}_{2}(3 \mathrm{~mL})$ at room temperature. After 1 hour, the reaction was quenched with saturated $\mathrm{NaHCO}_{3}(3$ $\mathrm{mL}$ ). The organic phase was separated, washed with brine, dried over anhydrous $\mathrm{MgSO}_{4}$, and concentrated under reduced pressure on the rotary evaporator. The crude product was purified by flash chromatography ( $5: 1$ hexanes:EtOAc) to provide the off-white solid $14(118 \mathrm{mg}, 0.28 \mathrm{mmol}, 93 \%) .14$ was recrystallized by the vapor diffusion method from toluene and hexanes.

${ }^{1} \mathrm{H}$ NMR $\left(400 \mathrm{MHz} ; \mathrm{CDCl}_{3}\right): \delta 9.19(\mathrm{t}, 1 \mathrm{H}, \mathrm{J}=2.1 \mathrm{~Hz}), 9.11(\mathrm{~d}, 2 \mathrm{H}, \mathrm{J}=2.1 \mathrm{~Hz})$, 7.47-7.44 (m, 1H), 7.39-7.28 (m, 3H), 6.68 (ddd, 1H, J = 5.7, 8.4, $11.4 \mathrm{~Hz}), 6.25$ (dd, 1H, $\mathrm{J}=1.8,6.3 \mathrm{~Hz}), 5.97(\mathrm{dd}, 1 \mathrm{H}, \mathrm{J}=2.6,11.4 \mathrm{~Hz}), 5.20(\mathrm{dd}, 1 \mathrm{H}, \mathrm{J}=2.8,9.5 \mathrm{~Hz}), 3.72-3.58$ $(\mathrm{m}, 2 \mathrm{H}), 3.23-3.15(\mathrm{~m}, 1 \mathrm{H}), 3.04(\mathrm{ddd}, 1 \mathrm{H}, \mathrm{J}=6.3,8.4,14.8 \mathrm{~Hz}), 1.44$ (d, 3H, J = 6.6 $\mathrm{Hz}) .{ }^{13} \mathrm{C} \mathrm{NMR}\left(100 \mathrm{MHz} ; \mathrm{CDCl}_{3}\right): \delta 165.6,162.2,148.9,144.2,140.9,140.2,134.0$, $129.6,129.1,127.8,125.9,125.6,124.8,122.7,78.6,71.0,38.1,34.2,15.6$. 


\section{X-ray structure of 14:}

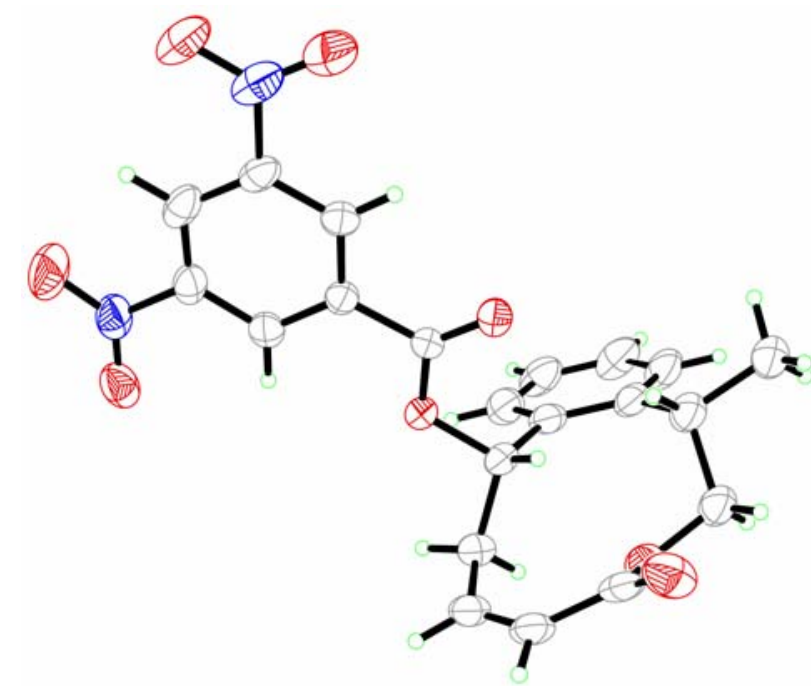

\section{Derivatization of $4(Z)$ to the 3,5-dinitrobenzoate 15 (X-ray structure).}

(Z)-2,4,7,8-tetrahydro-2-methyl-4-oxo-1H-benzo[d]oxecin-8-yl 3,5-dinitrobenzoate (15):<smiles>COC(C)Cc1ccccc1C(O)C/C=C\C(=O)O</smiles>

$4(Z)$

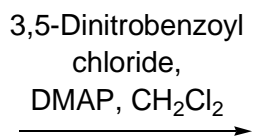

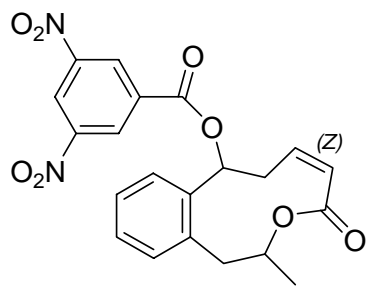

15

Compound 15 was prepared from $4(Z)$ by following the procedure for the preparation of $\mathbf{1 4}$ and was purified by flash chromatography (5:1 hexanes:EtOAc) to provide an off-white solid (121 mg, $0.29 \mathrm{mmol}, 95 \%$ ). Recrystallization of 15 was achieved by vapor diffusion from toluene and hexanes

${ }^{1} \mathrm{H}$ NMR $\left(400 \mathrm{MHz} ; \mathrm{CDCl}_{3}\right): \delta 9.16(\mathrm{t}, 1 \mathrm{H}, \mathrm{J}=2.1 \mathrm{~Hz}), 9.07(\mathrm{~d}, 2 \mathrm{H}, \mathrm{J}=2.1 \mathrm{~Hz})$, 7.49-7.43 (m, 1H), 7.31-7.18 (m, 3H), $6.99(\mathrm{dd}, 1 \mathrm{H}, \mathrm{J}=6.1,8.3 \mathrm{~Hz}), 6.05(\mathrm{ddd}, 1 \mathrm{H}, \mathrm{J}=$ 4.9, 6.4, $12.6 \mathrm{~Hz}), 5.66(\mathrm{ddd}, 1 \mathrm{H}, \mathrm{J}=1.7,2.7,12.8 \mathrm{~Hz}), 5.28-5.18(\mathrm{~m}, 1 \mathrm{H}), 3.57(\mathrm{dd}, 1 \mathrm{H}$, $\mathrm{J}=11.6,13.8 \mathrm{~Hz}), 3.40-3.24(\mathrm{~m}, 2 \mathrm{H}), 2.85(\mathrm{dd}, 1 \mathrm{H}, \mathrm{J}=3.3,13.9 \mathrm{~Hz}), 1.56(\mathrm{~d}, 3 \mathrm{H}, \mathrm{J}=6.3$ $\mathrm{Hz}) .{ }^{13} \mathrm{C} \mathrm{NMR}\left(100 \mathrm{MHz} ; \mathrm{CDCl}_{3}\right): \delta 165.0,161.8,148.8,137.0,136.9,136.7,134.1$, $131.3,129.7,129.6,128.0,126.8,123.1,122.6,73.7,72.1,40.5,36.9,20.8$. 


\section{$\mathrm{X}$-ray structure of 15:}

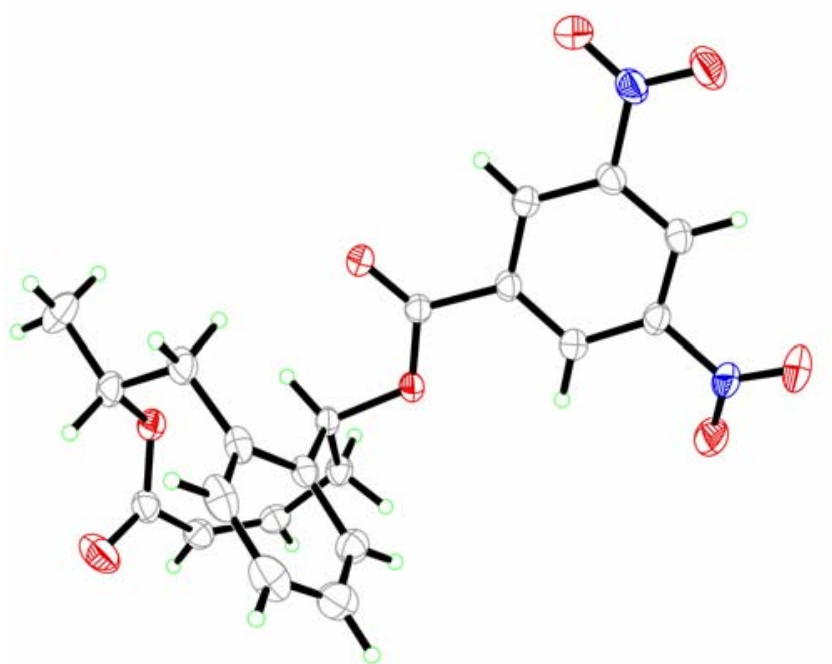

Derivatization of 7 to the 3,5-dinitrobenzoate 16 (X-ray structure).

3,5-Dinitro-benzoic acid 8-methyl-10-oxo-5,7,8,10,13,14-hexahydro-6H-9-oxabenzocyclododecen-14-yl ester (16):

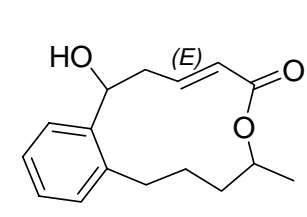

7
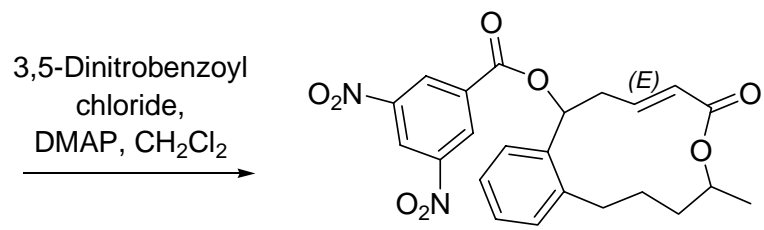

16

Compound 16 was prepared from 7 by following the procedure for the preparation of $\mathbf{1 4}$ and was purified by flash chromatography (5:1 hexanes:EtOAc) to provide an off-white solid ( $84 \mathrm{mg}, 0.2 \mathrm{mmol}, 92 \%)$. Recrystallization of $\mathbf{1 6}$ was achieved by vapor diffusion from toluene and hexanes

${ }^{1} \mathrm{H}$ NMR $\left(400 \mathrm{MHz} ; \mathrm{CDCl}_{3}\right): \delta 9.24(\mathrm{t}, 1 \mathrm{H}, \mathrm{J}=2.2 \mathrm{~Hz}), 9.14(\mathrm{~d}, 2 \mathrm{H}, \mathrm{J}=2.1 \mathrm{~Hz})$, 7.52-7.42 (br m, 1H), 7.27-7.18 (br m, 2H), 7.17-7.08 (br m, 1H), 6.85-6.72 (br m, 1H), 6.29 (br d, 1H, J = 9.3 Hz), 5.65 (br d, 1H, J = 16.0 Hz), 5.07 (br s, 1H), 3.15 (br s, 1H), 2.90-2.70 (m, 3H), 2.24-2.10 (br m, 1H), 1.95-1.84 (br m, 1H), 1.55 (br s, 1H), 1.33 (d, $3 \mathrm{H}, \mathrm{J}=6.4 \mathrm{~Hz}), 1.30-1.20$ (br m, 1H). ${ }^{13} \mathrm{C} \mathrm{NMR}\left(100 \mathrm{MHz} ; \mathrm{CDCl}_{3}\right): \delta 174.1,161.9$, $149.0,141.6,134.0,129.7,128.9,128.3,127.0,126.3,125.9,122.9,74.6,40.1,35.0$, $31.2,24.8,20.4$. 


\section{$\mathrm{X}$-ray structure of 16:}

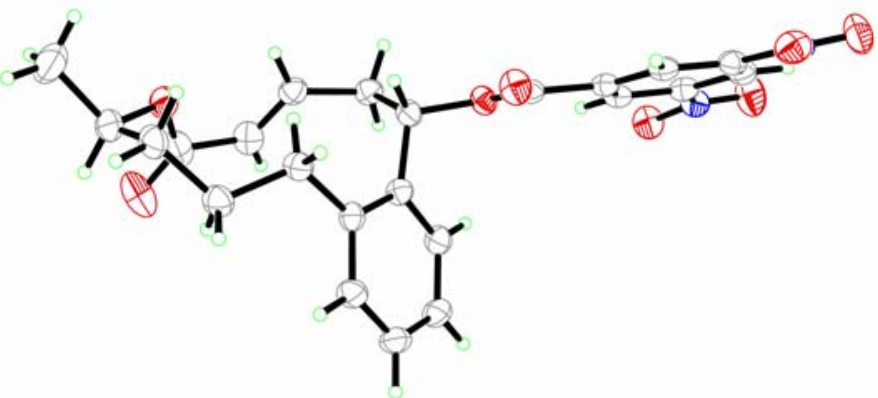

Scheme 11. Preparation of $(5 R, 11 R)-17$ and $(5 S, 11 R)-17$ from lactone 10.

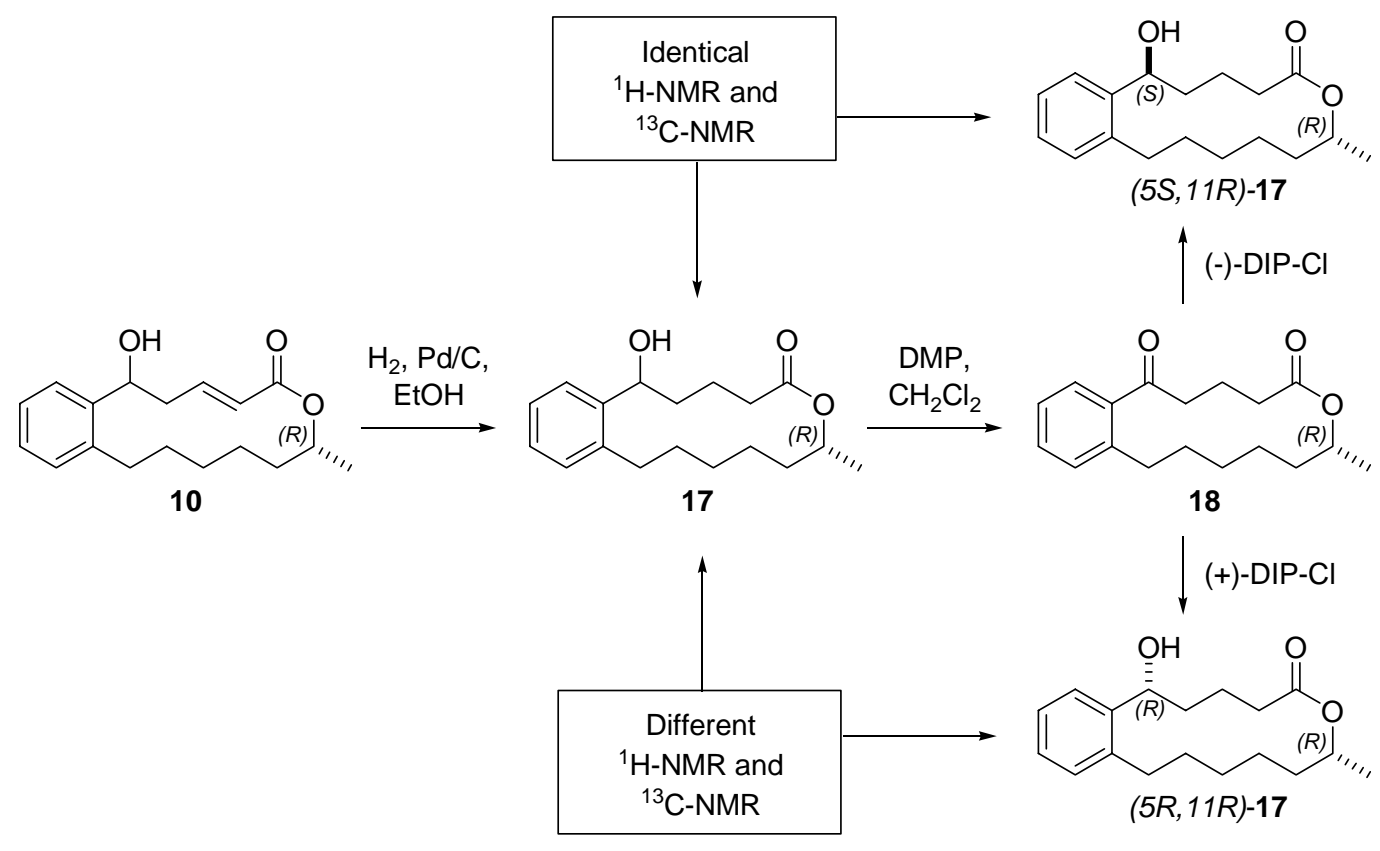

Hydrogenation of lactone 10 with $\mathrm{H}_{2}-\mathrm{Pd} / \mathrm{C}$ provided lactone 17. A Dess-Martin periodinane oxidation of the stereogenic alcohol within lactone $\mathbf{1 7}$ resulted in ketone $\mathbf{1 8}$. Ketone 18 was then subjected to an asymmetric reduction with DIP-Chloride to obtain both diastereomers $(5 R, 11 R)-17$ and $(5 S, 11 R)-17$.

The asymmetric reduction of ketone 18 with (-)-DIP-Chloride provided (5S,11R)-17, which has spectral data $\left({ }^{1} \mathrm{H}\right.$ and $\left.{ }^{13} \mathrm{C} N M R\right)$ that is identical to that of alcohol 17. Additionally, the diastereomer $(5 R, 11 R)-17$ obtained from the asymmetric reduction with (+)-DIP-Chloride has spectral data that differs from that of 17. This, therefore, indicates that the alcohol stereocenter formed in the intramolecular vinylgous aldol reaction has the $S$ configuration and that the relationship between the two tetrahedral stereocenters within lactone $\mathbf{1 0}$ is anti, as drawn in the scheme above. 


\section{5-Hydroxy-11-(R)-methyl-5,6,7,8,11,12,13,14,15,16-decahydro-10-oxa-} benzocyclotetradecen-9-one (17):

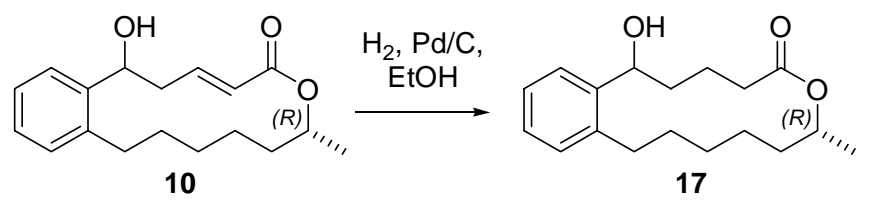

Compound 17 was prepared from 10 by following the procedure for the preparation of 12 and was purified by flash chromatography (5:1 hexanes:EtOAc) to provide a colorless oil $(0.30 \mathrm{~g}, 1.0 \mathrm{mmol}, 96 \%)$.

${ }^{1} \mathrm{H}$ NMR $\left(400 \mathrm{MHz} ; \mathrm{CDCl}_{3}\right): \quad \delta$ 7.50-7.45 $(\mathrm{m}, 1 \mathrm{H}), 7.23-7.13(\mathrm{~m}, 3 \mathrm{H})$, 5.09-4.98 (m, 2H), 2.70 (ddd, $1 \mathrm{H}, \mathrm{J}=6.6,9.4,14.0 \mathrm{~Hz}$ ), 2.59 (ddd, $1 \mathrm{H}, \mathrm{J}=3.4,8.4,15.6$ $\mathrm{Hz}$ ), 2.49 (ddd, $1 \mathrm{H}, \mathrm{J}=5.5,9.2,14.5 \mathrm{~Hz}$ ), 2.27 (ddd, $1 \mathrm{H}, \mathrm{J}=3.4,9.3,15.6 \mathrm{~Hz}$ ), 2.06-1.84 $(\mathrm{m}, 3 \mathrm{H}), 1.76-1.23(\mathrm{~m}, 10 \mathrm{H}), 1.21(\mathrm{~d}, 3 \mathrm{H}, \mathrm{J}=6.6 \mathrm{~Hz}) .{ }^{13} \mathrm{C} \mathrm{NMR}\left(100 \mathrm{MHz} ; \mathrm{CDCl}_{3}\right): \delta$ $173.1,143.2,138.5,129.2,127.5,126.5,125.5,70.5,69.3,38.0,33.8,33.4,29.9,29.8$, 27.2, 22.2, 21.7, 19.0. IR $\left(\mathrm{cm}^{-1}\right): 3436,2935,2860,1728,1238,1063,757 . \quad[\alpha]_{\mathrm{D}}-27.4$ (c $0.24,26^{\circ} \mathrm{C}, \mathrm{CH}_{2} \mathrm{Cl}_{2}$ ). HRMS (ESI) $\mathrm{m} / \mathrm{z}$ calc'd for $\mathrm{C}_{18} \mathrm{H}_{26} \mathrm{O}_{3} \mathrm{Na}[\mathrm{M}+\mathrm{Na}]^{+}$: 313.1779 ; found: 313.1769 .

11-Methyl-7,8,11,12,13,14,15,16-octahydro-6H-10-oxa-benzocyclotetradecene-5,9dione (18):

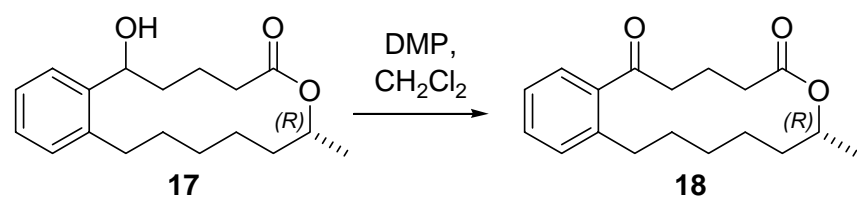

Compound $\mathbf{1 8}$ was prepared from $\mathbf{1 7}$ by following the procedure for the preparation of $4 \mathbf{a}$ and was purified by flash chromatography (10:1 hexanes:EtOAc) to provide a colorless oil $(0.16 \mathrm{~g}, 78 \%)$.

${ }^{1} \mathrm{H}$ NMR $\left(400 \mathrm{MHz} ; \mathrm{CDCl}_{3}\right): \delta$ 7.35-7.29 (m, $\left.1 \mathrm{H}\right), 7.26-7.22(\mathrm{~m}, 2 \mathrm{H}), 7.20-7.15$ (m, 1H), 5.05-4.97 (m, 1H), 3.04 (ddd, 1H, J = 5.6, 7.1, 19.3 Hz), 2.89-2.76 (m, 2H), 2.63 (ddd, $1 \mathrm{H}, \mathrm{J}=3.5,9.5,15.9 \mathrm{~Hz}), 2.57$ (ddd, $1 \mathrm{H}, \mathrm{J}=5.8,7.8,13.9 \mathrm{~Hz}$ ), 2.39 (ddd, $1 \mathrm{H}, \mathrm{J}=$ 3.6, 8.3, 15.9 Hz), 2.19-2.07 (m, 1H), 2.03-1.92 (m, 1H), 1.67-1.18 (m, 8H), $1.18(\mathrm{~d}, 3 \mathrm{H}$, $\mathrm{J}=6.5 \mathrm{~Hz}) .{ }^{13} \mathrm{C}$ NMR $\left(100 \mathrm{MHz} ; \mathrm{CDCl}_{3}\right): \delta 207.1,173.0,141.8,139.6,130.3,130.2$, 126.0, 125.7, 70.8, 41.6, 33.8, 33.2, 30.6, 30.5, 27.2, 22.6, 19.4, 18.8. IR $\left(\mathrm{cm}^{-1}\right): 2934$, $2858,1726,1695,1255,758$. $[\alpha]_{\mathrm{D}}+12.1\left(c 1.14,26^{\circ} \mathrm{C}, \mathrm{CH}_{2} \mathrm{Cl}_{2}\right)$. HRMS (ESI) $\mathrm{m} / \mathrm{z}$ calc'd for $\mathrm{C}_{18} \mathrm{H}_{24} \mathrm{O}_{3} \mathrm{Na}[\mathrm{M}+\mathrm{Na}]^{+}$: 311.1623 ; found: 311.1614 .

5-(S)-Hydroxy-11-(R)-methyl-5,6,7,8,11,12,13,14,15,16-decahydro-10-oxabenzocyclotetradecen-9-one [(5S,11R)-17]

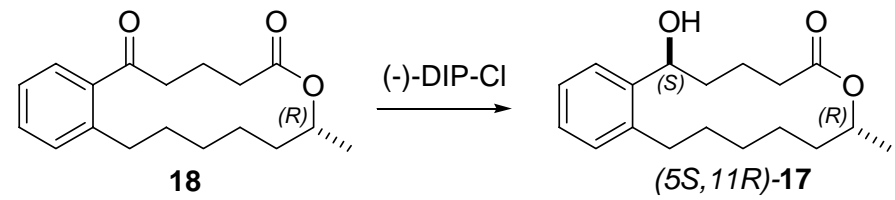


A freshly prepared solution ${ }^{7}$ of (-)-DIP-Cl (93 uL of 1.65 M sol. in DME, 0.15 mmol) was added to a solution of $18(22 \mathrm{mg}, 0.076 \mathrm{mmol})$ in THF $(1 \mathrm{~mL})$ at room temperature. After allowing the reaction to stir overnight $(18 \mathrm{hr})$, the reaction mixture was diluted with $\mathrm{Et}_{2} \mathrm{O}(5 \mathrm{~mL})$ and then treated with $6 \mathrm{M} \mathrm{NaOH}(1 \mathrm{~mL})$. After vigorous stirring for $5 \mathrm{~min}$, the organic phase was separated, washed with brine, dried over anhydrous $\mathrm{MgSO}_{4}$, and concentrated under reduced pressure on the rotary evaporator. The crude product was purified by flash chromatography ( $5: 1$ hexanes:EtOAc) to provide exclusively $(5 S, 11 R)-17$ (17 mg, $0.06 \mathrm{mmol}, 78 \%)$.

${ }^{1} \mathrm{H}$ NMR $\left(400 \mathrm{MHz} ; \mathrm{CDCl}_{3}\right): \delta$ 7.50-7.45 (m, 1H), 7.23-7.13 (m, 3H), 5.09-4.99 ( $\mathrm{m}, 2 \mathrm{H}), 2.70(\mathrm{ddd}, 1 \mathrm{H}, \mathrm{J}=6.6,9.4,14.0 \mathrm{~Hz}), 2.60(\mathrm{ddd}, 1 \mathrm{H}, \mathrm{J}=3.4,8.4,15.6 \mathrm{~Hz}), 2.50$ (ddd, $1 \mathrm{H}, \mathrm{J}=5.5,9.2,14.5 \mathrm{~Hz}), 2.27(\mathrm{ddd}, 1 \mathrm{H}, \mathrm{J}=3.4,9.3,15.6 \mathrm{~Hz}), 2.07-1.85(\mathrm{~m}, 2 \mathrm{H})$, $1.76-1.24(\mathrm{~m}, 11 \mathrm{H}), 1.21(\mathrm{~d}, 3 \mathrm{H}, \mathrm{J}=6.6 \mathrm{~Hz}) .{ }^{13} \mathrm{C} \mathrm{NMR}\left(100 \mathrm{MHz} ; \mathrm{CDCl}_{3}\right): \delta 173.1$, $143.2,138.5,129.2,127.5,126.5,125.5,70.5,69.3,38.0,33.8,33.4,29.9,29.8,27.2$, 22.2, 21.7, 19.0. IR $\left(\mathrm{cm}^{-1}\right): 3436,2935,2861,1728,1238,1063,757$. [ $\left.\alpha\right]_{\mathrm{D}}-31.6(c$ $0.75,26^{\circ} \mathrm{C}, \mathrm{CH}_{2} \mathrm{Cl}_{2}$ ). HRMS (ESI) $\mathrm{m} / \mathrm{z}$ calc'd for $\mathrm{C}_{18} \mathrm{H}_{26} \mathrm{O}_{3} \mathrm{Na}[\mathrm{M}+\mathrm{Na}]^{+}$: 313.1779 ; found: 313.1782 .

\section{5-(R)-Hydroxy-11-(R)-methyl-5,6,7,8,11,12,13,14,15,16-decahydro-10-oxa- benzocyclotetradecen-9-one [(5R,11R)-17]:}

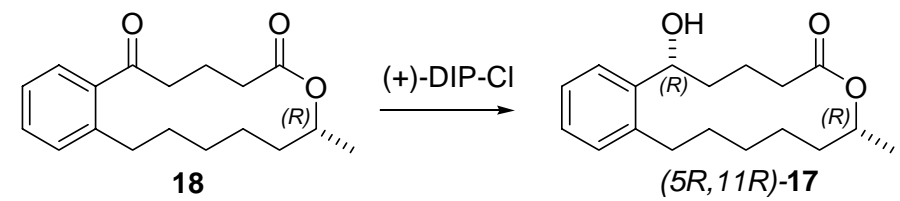

(+)-DIP-Cl (49 mg, $0.15 \mathrm{mmol})$ was added to a solution of 18 (22 mg, 0.076 $\mathrm{mmol})$ in THF $(1 \mathrm{~mL})$ at room temperature. After allowing the reaction to stir overnight $(18 \mathrm{hr})$, the reaction mixture was diluted with $\mathrm{Et}_{2} \mathrm{O}(5 \mathrm{~mL})$ and then treated with $6 \mathrm{M}$ $\mathrm{NaOH}(1 \mathrm{~mL})$. After vigorous stirring for $5 \mathrm{~min}$, the organic phase was separated, washed with brine, dried over anhydrous $\mathrm{MgSO}_{4}$, and concentrated under reduced pressure on the rotary evaporator. The crude product was purified by flash chromatography $(5: 1$ hexanes:EtOAc) to provide exclusively $(5 R, 11 R)-17(18 \mathrm{mg}, 0.06$ mmol, 82\%).

${ }^{1} \mathrm{H}$ NMR $\left(400 \mathrm{MHz} ; \mathrm{CDCl}_{3}\right): \delta 7.48(\mathrm{dd}, 1 \mathrm{H}, \mathrm{J}=1.7,7.6 \mathrm{~Hz}), 7.20(\mathrm{dt}, 1 \mathrm{H}, \mathrm{J}=$ 1.7, $7.2 \mathrm{~Hz}), 7.16(\mathrm{dt}, 1 \mathrm{H}, \mathrm{J}=1.7,7.5 \mathrm{~Hz}), 7.12(\mathrm{dd}, 1 \mathrm{H}, \mathrm{J}=1.7,7.4 \mathrm{~Hz}), 5.12-5.04(\mathrm{~m}$, $1 \mathrm{H}), 4.78-4.72(\mathrm{~m}, 1 \mathrm{H}), 2.81(\mathrm{~d}, 1 \mathrm{H}, \mathrm{J}=3.5 \mathrm{~Hz}), 2.71-2.62(\mathrm{~m}, 1 \mathrm{H}), 2.55(\mathrm{ddd}, 1 \mathrm{H}, \mathrm{J}=$ 2.0, 8.0, $16.5 \mathrm{~Hz}), 2.40-2.24(\mathrm{~m}, 2 \mathrm{H}), 2.17-2.04(\mathrm{~m}, 1 \mathrm{H}), 1.92-1.71(\mathrm{~m}, 3 \mathrm{H}), 1.65-1.53$ $(\mathrm{m}, 3 \mathrm{H}), 1.50-1.26(\mathrm{~m}, 5 \mathrm{H}), 1.22(\mathrm{~d}, 3 \mathrm{H}, \mathrm{J}=6.6 \mathrm{~Hz}) .{ }^{13} \mathrm{C} \mathrm{NMR}\left(100 \mathrm{MHz} ; \mathrm{CDCl}_{3}\right): \delta$ 1.75.3, 142.3, 138.7, 129.3, 127.4, 126.6, 126.0, 71.3, 68.0, 39.7, 34.4, 32.8, 30.4, 29.3, 27.1, 20.3, 19.3. IR $\left(\mathrm{cm}^{-1}\right): 3479,2929,2866,1725,1270,751 .[\alpha]_{\mathrm{D}}+22.7(c 0.28$, $26^{\circ} \mathrm{C}, \mathrm{CH}_{2} \mathrm{Cl}_{2}$ ). HRMS (ESI) $\mathrm{m} / \mathrm{z}$ calc'd for $\mathrm{C}_{18} \mathrm{H}_{26} \mathrm{O}_{3} \mathrm{Na}[\mathrm{M}+\mathrm{Na}]^{+}: 313.1779$; found: 313.1784 .

\footnotetext{
${ }^{7}$ (-)-DIP-Cl was prepared according to: Zhao, M.; King, A. O.; Larsen, R. D.; Verhoeven, T. R.; Reider, P. J., Tetrahedral Lett. 1997, 38, 2641.
} 


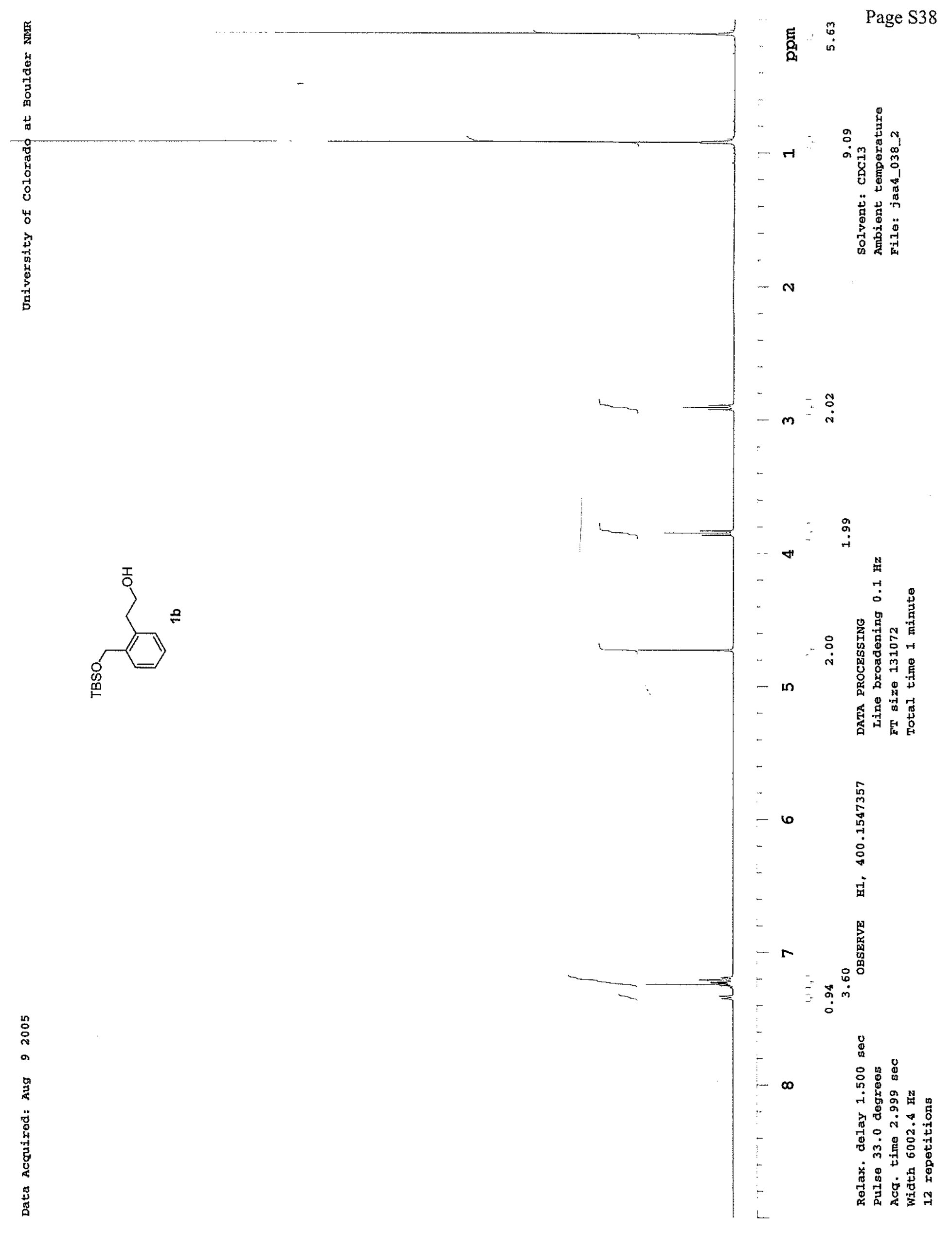




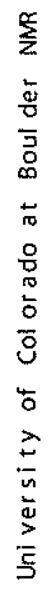
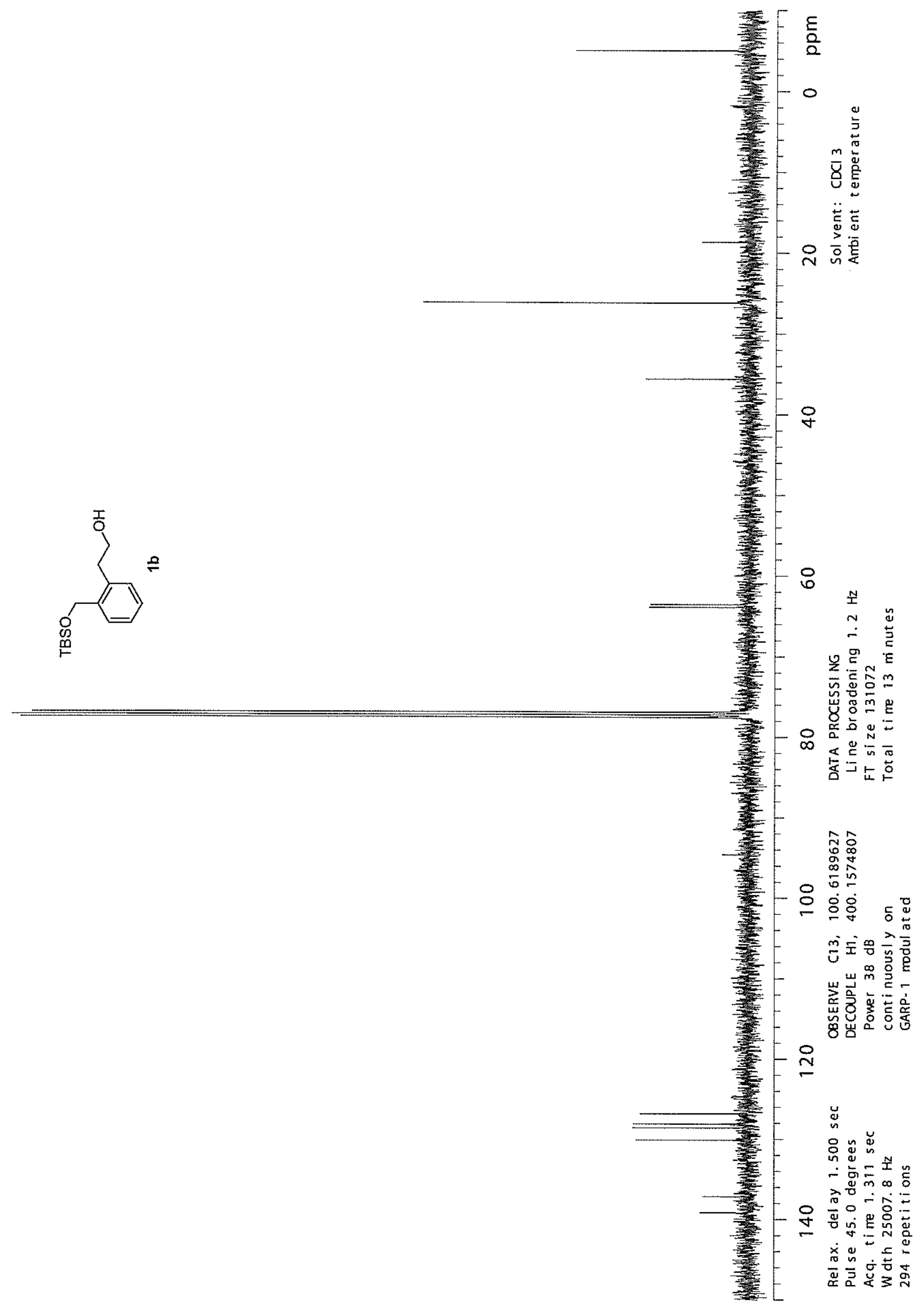


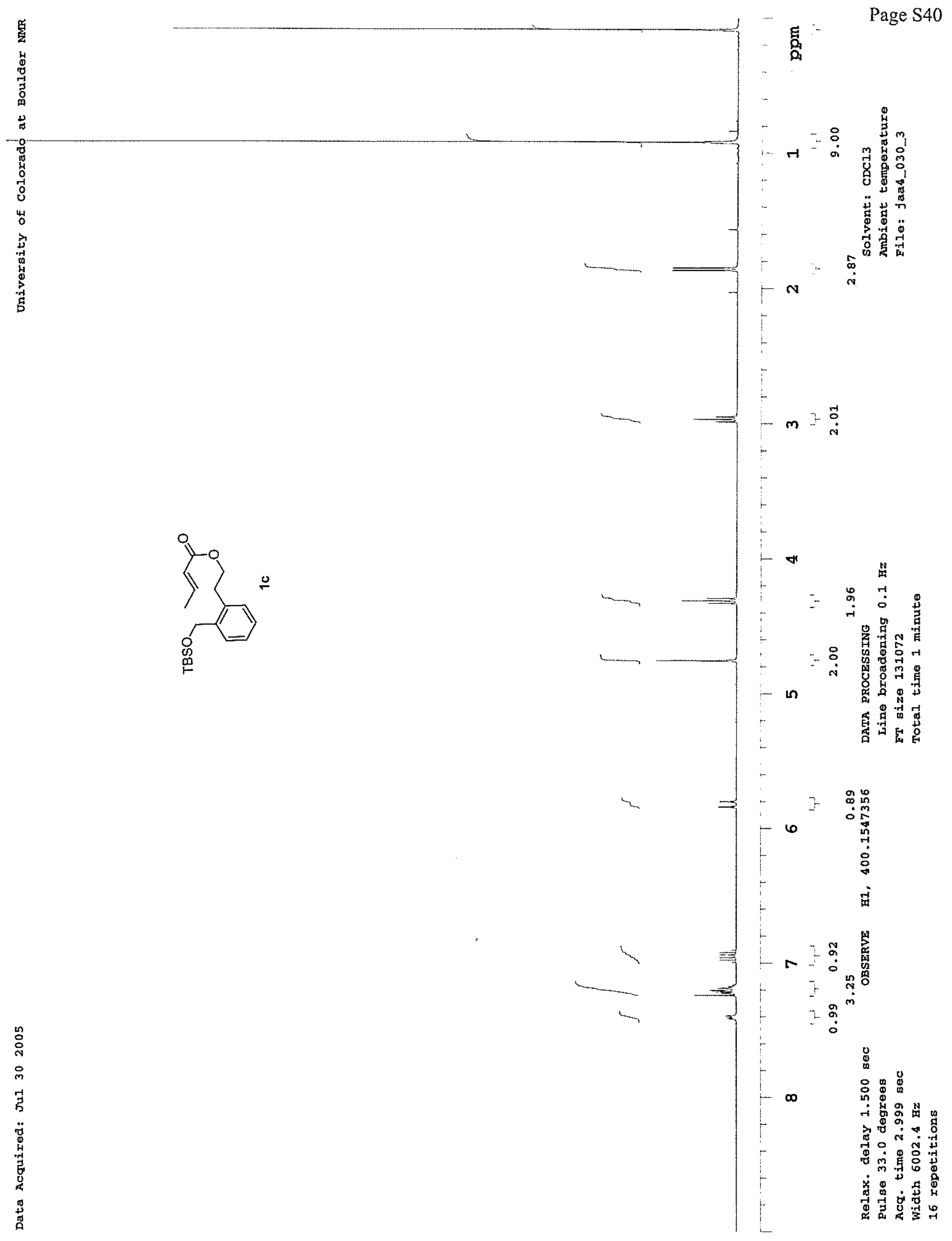


Page S41

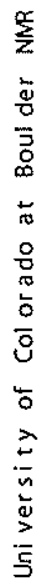

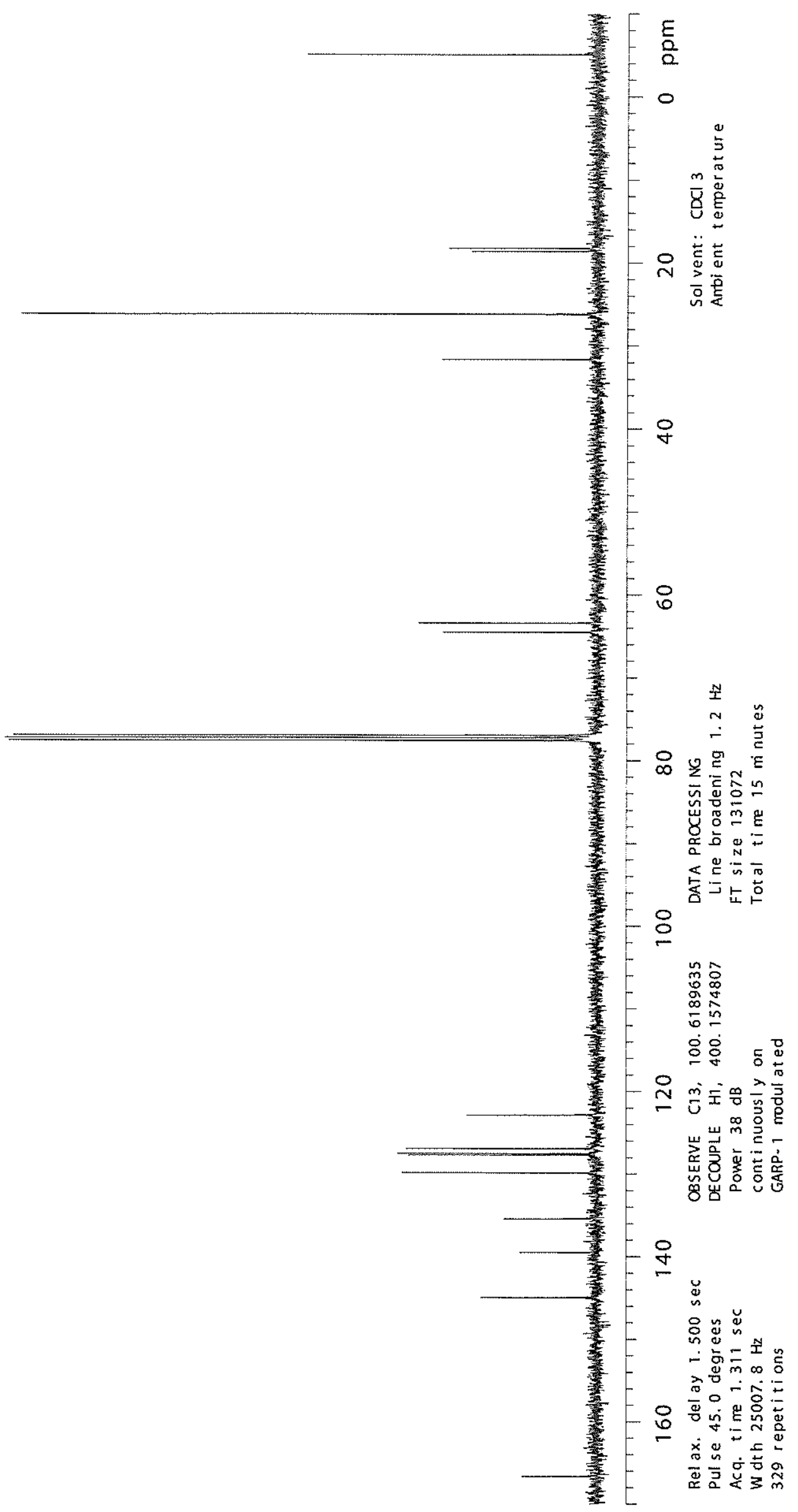




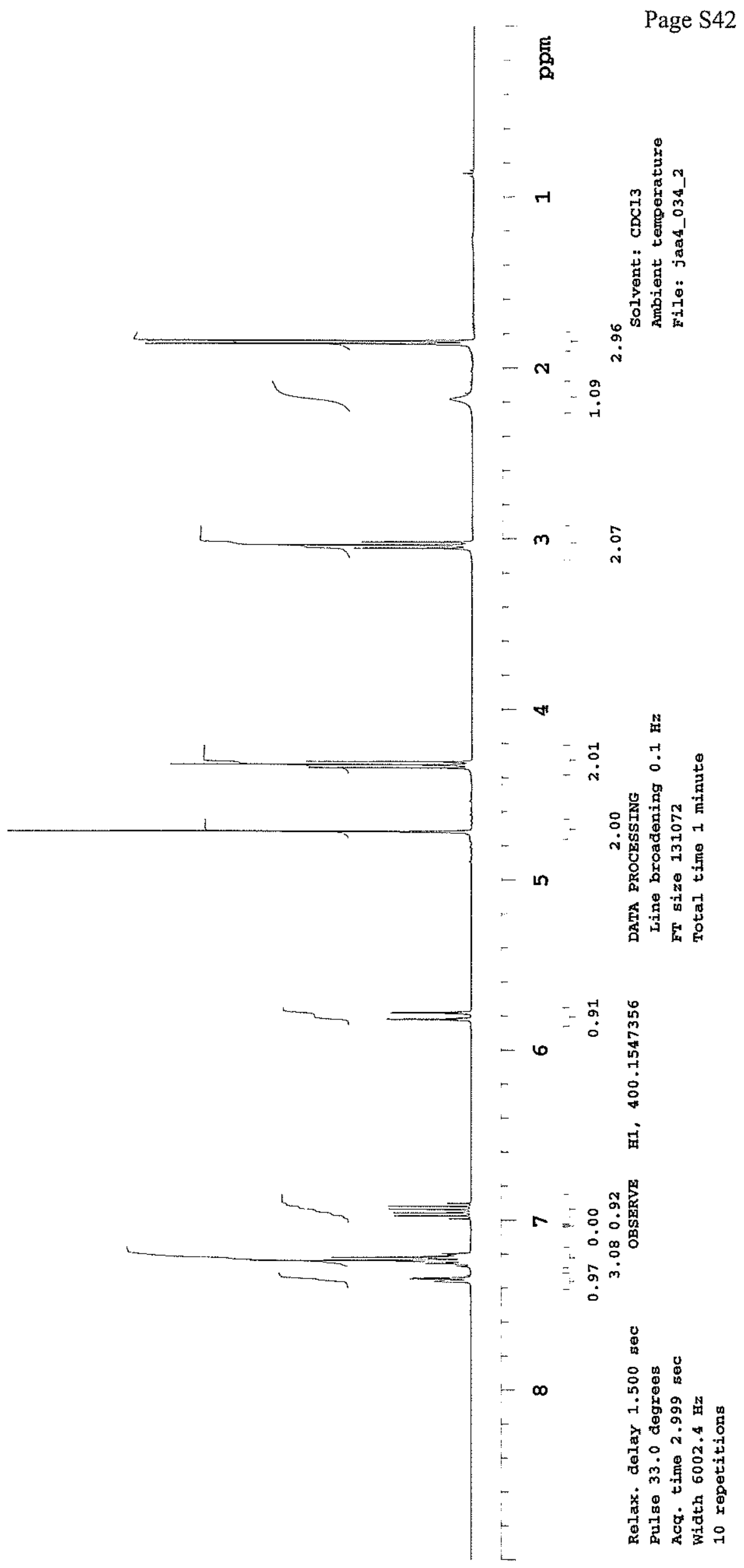




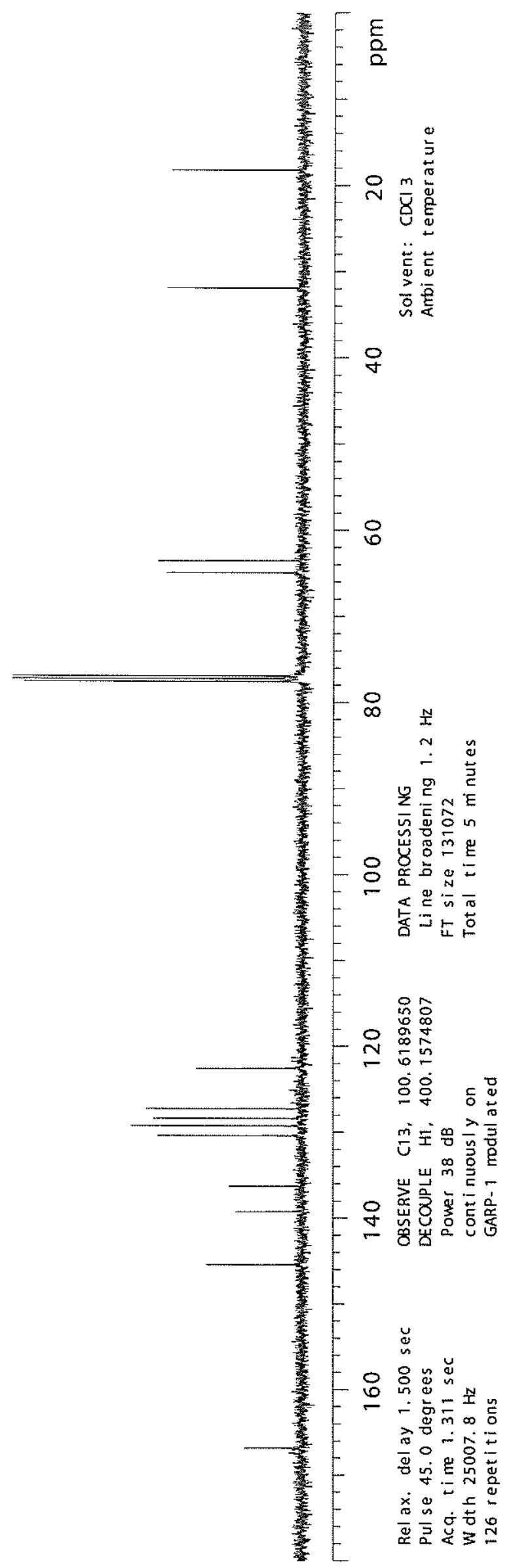




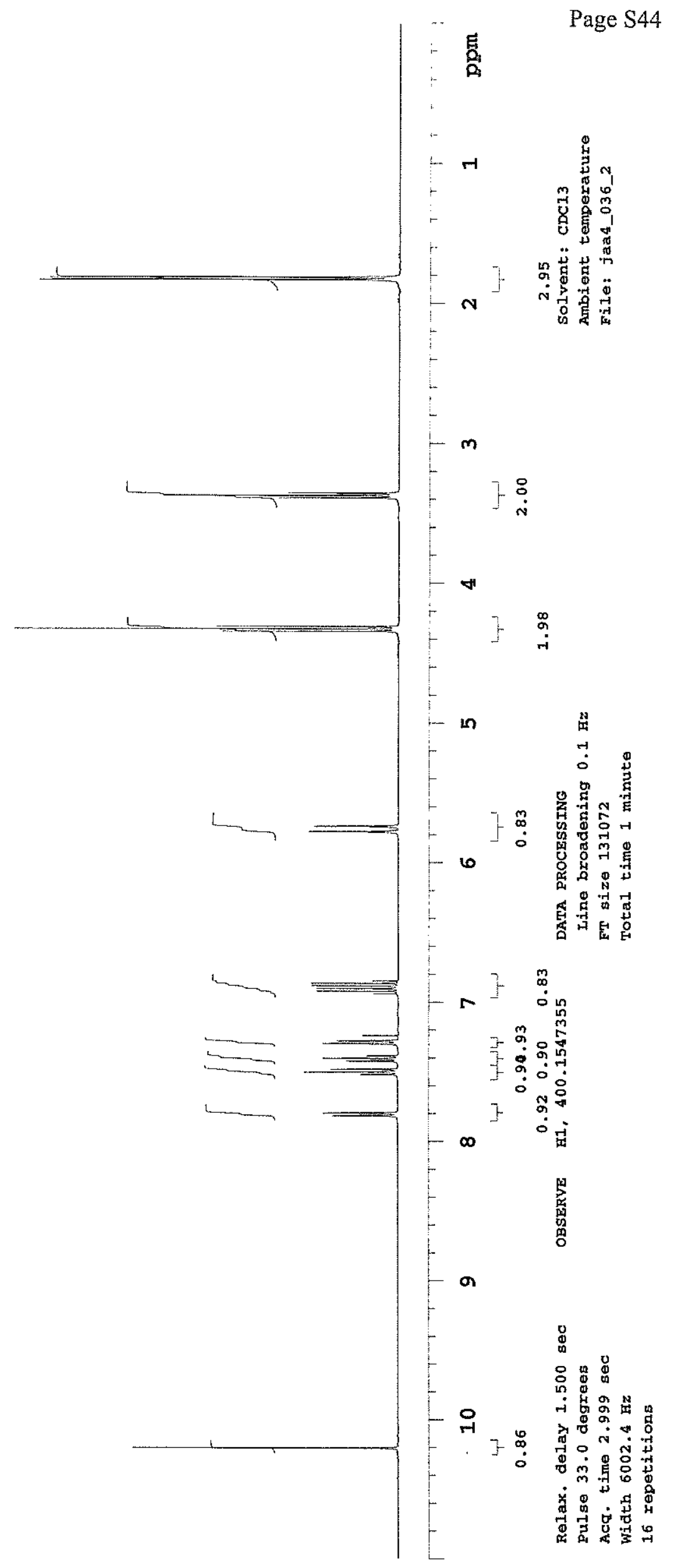




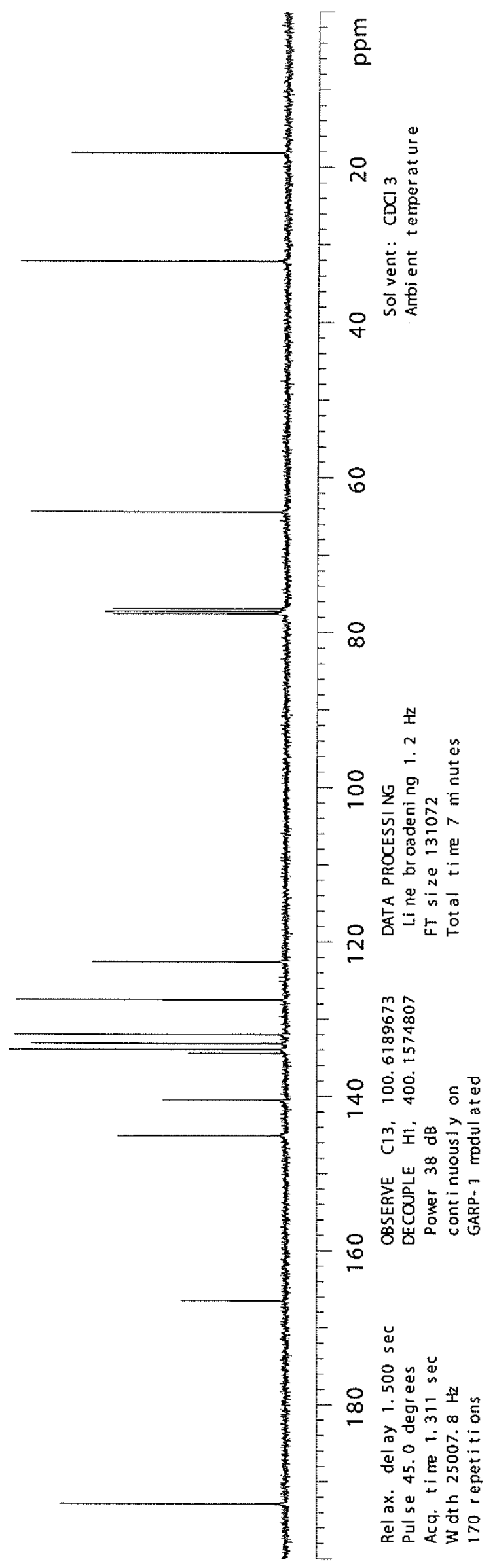




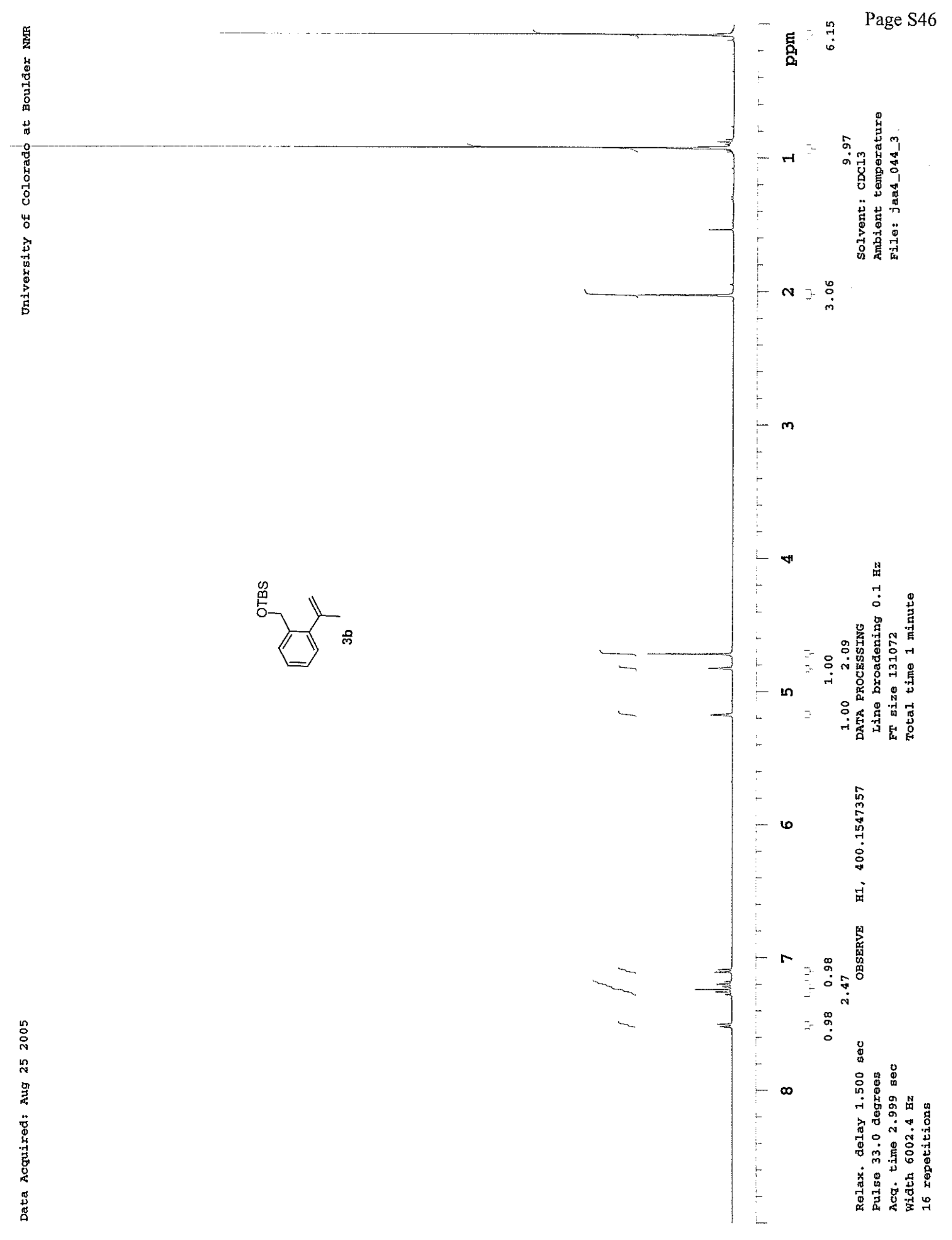




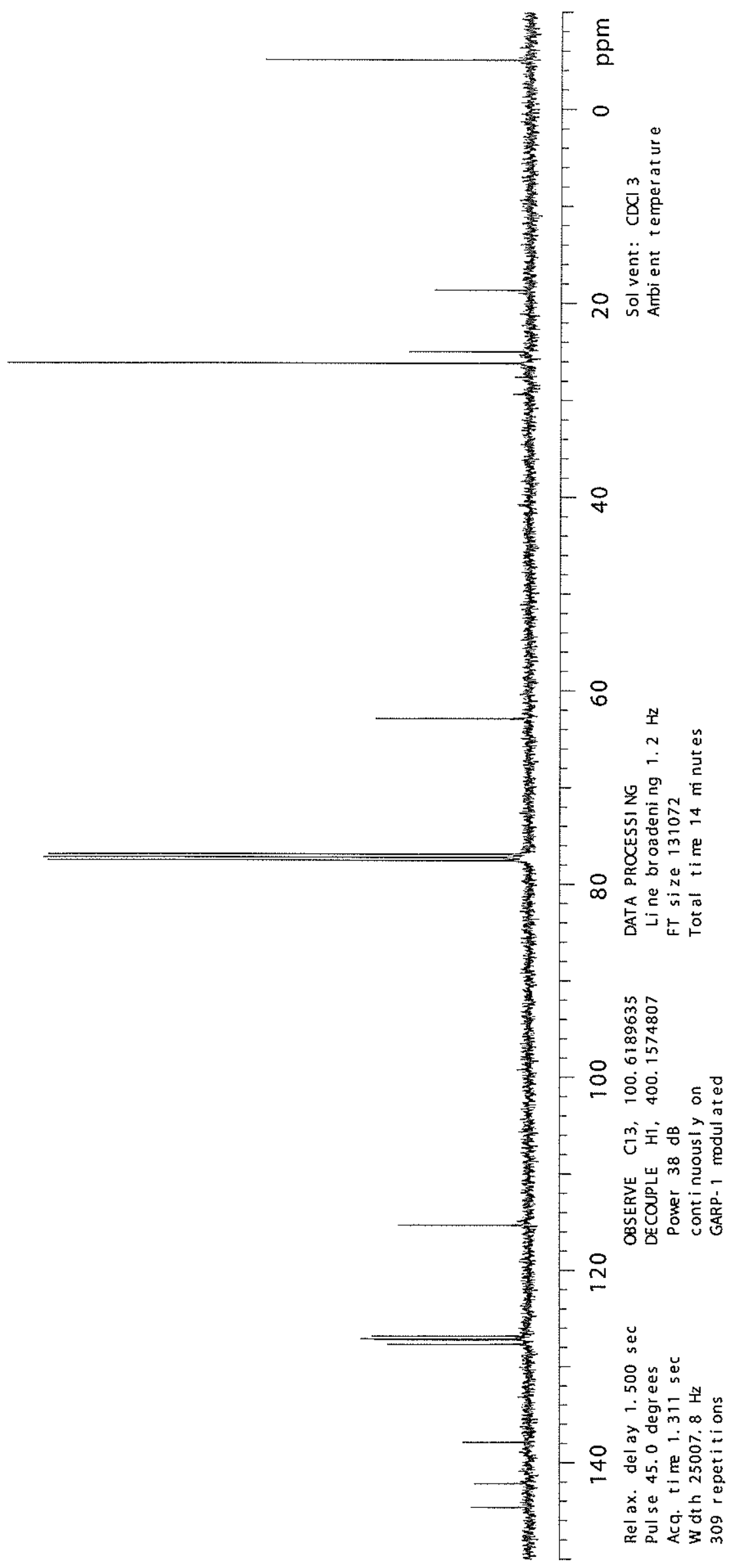




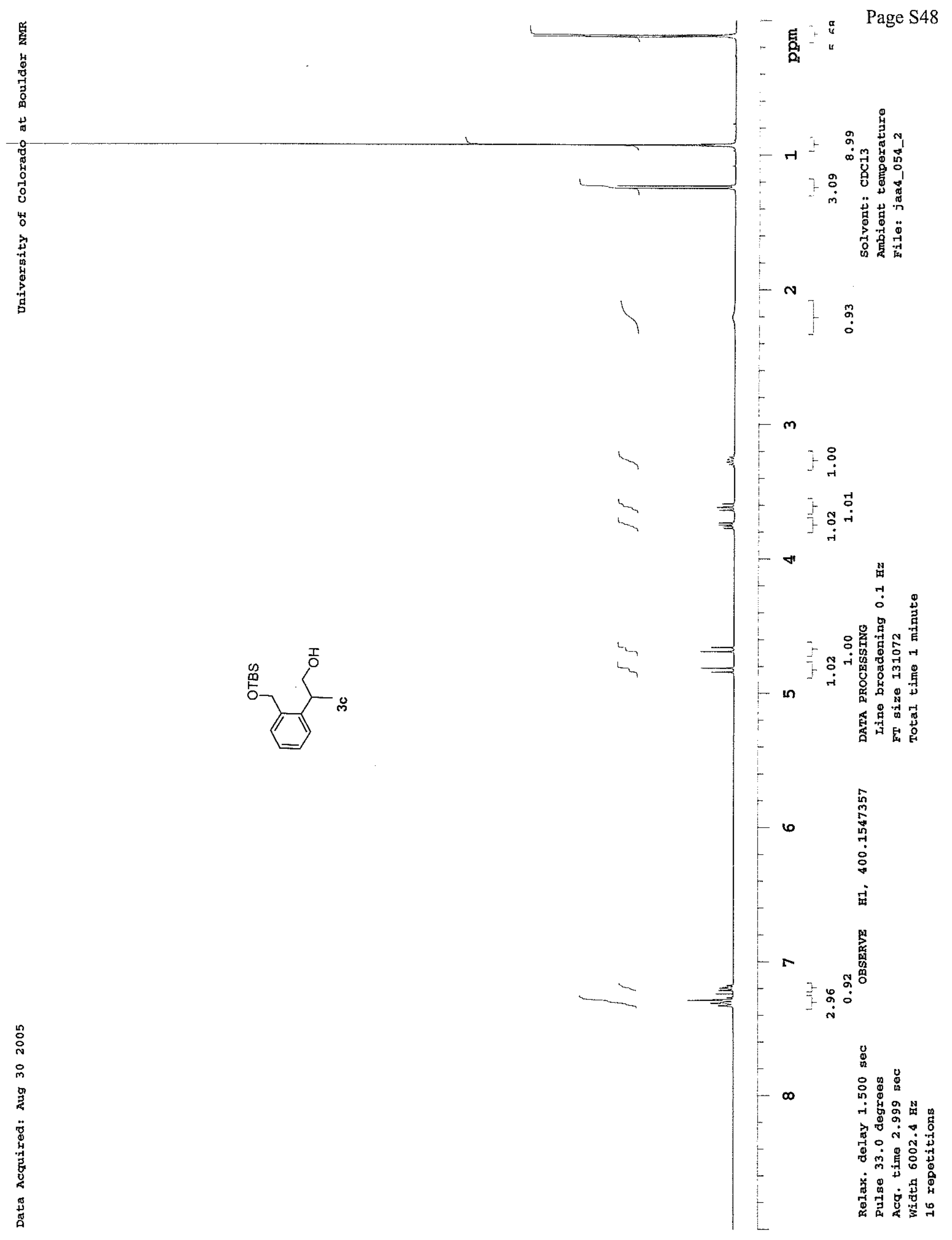




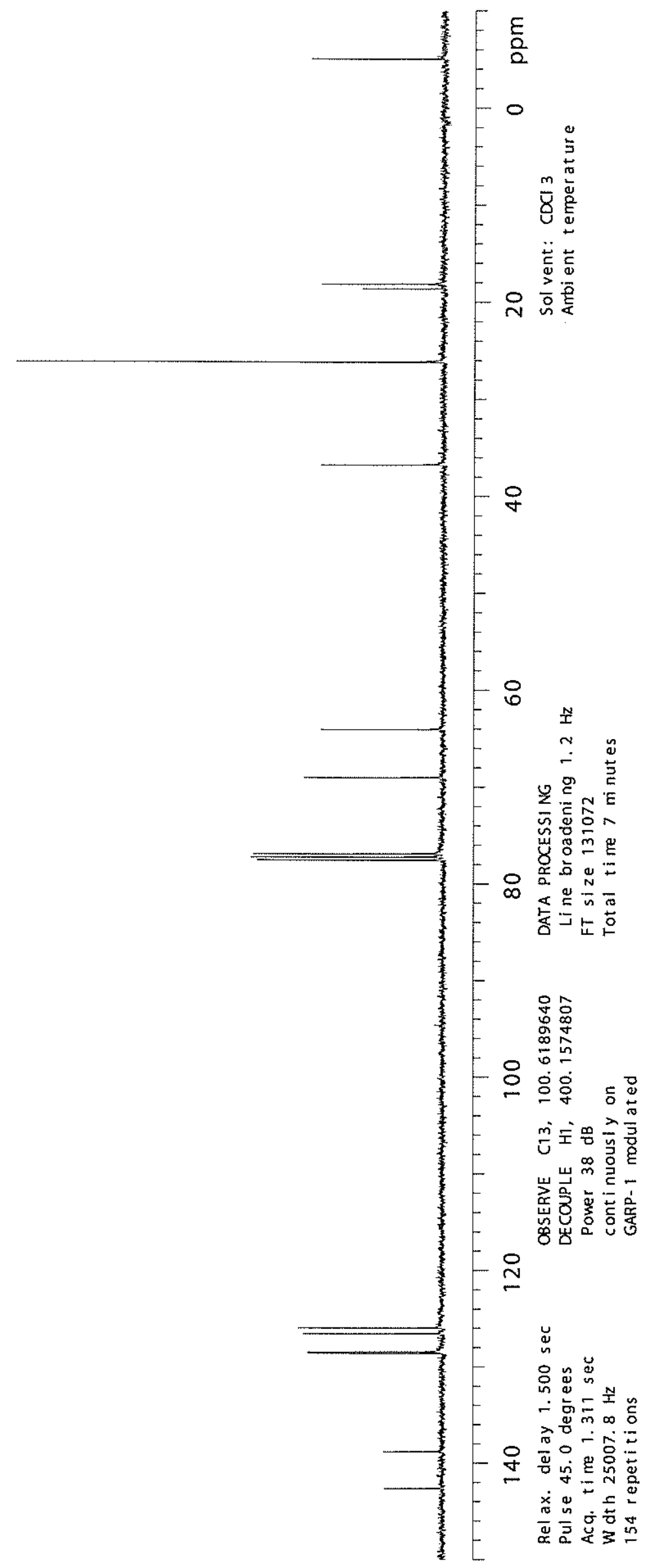




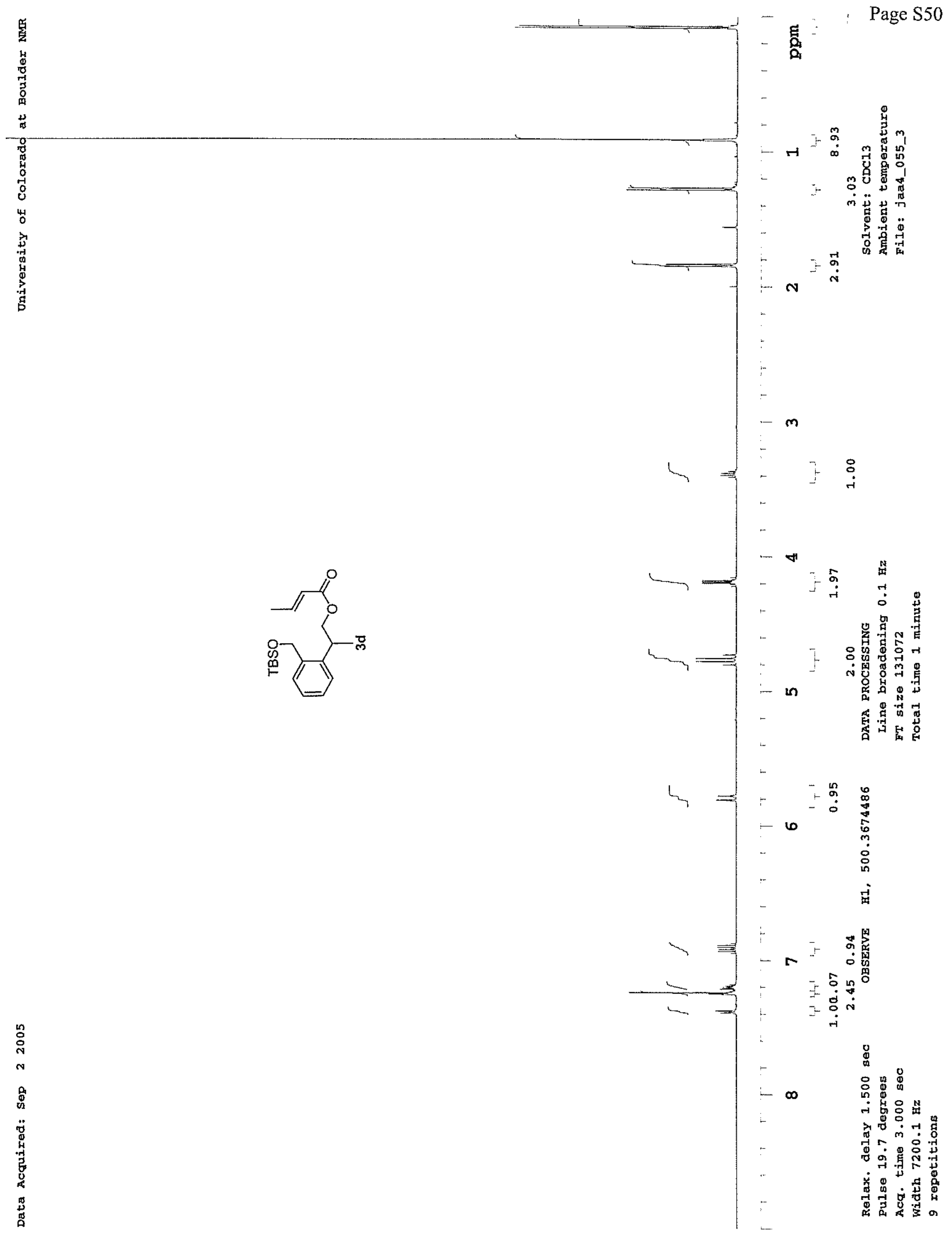


Page S51

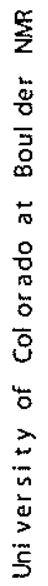
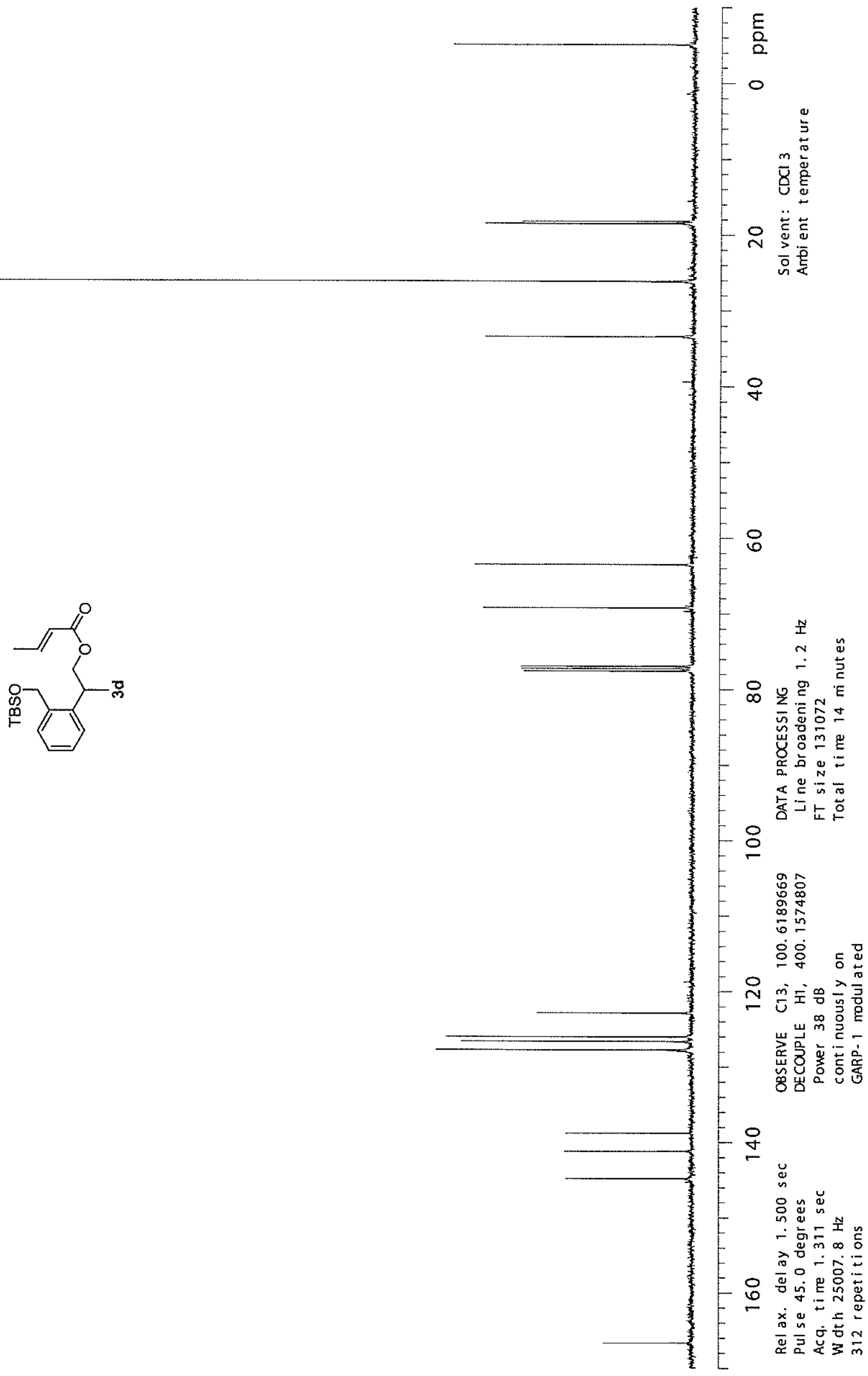


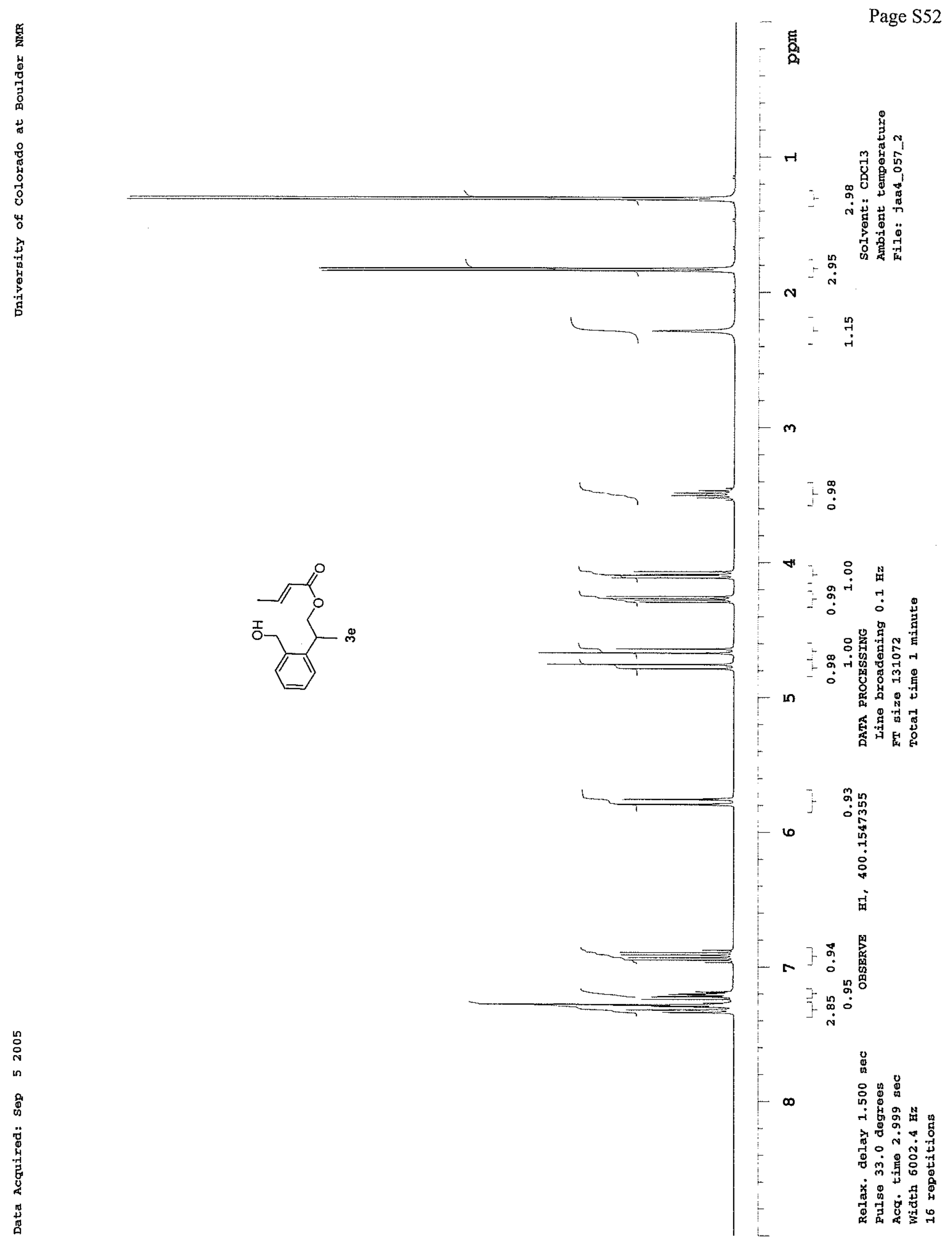


s.

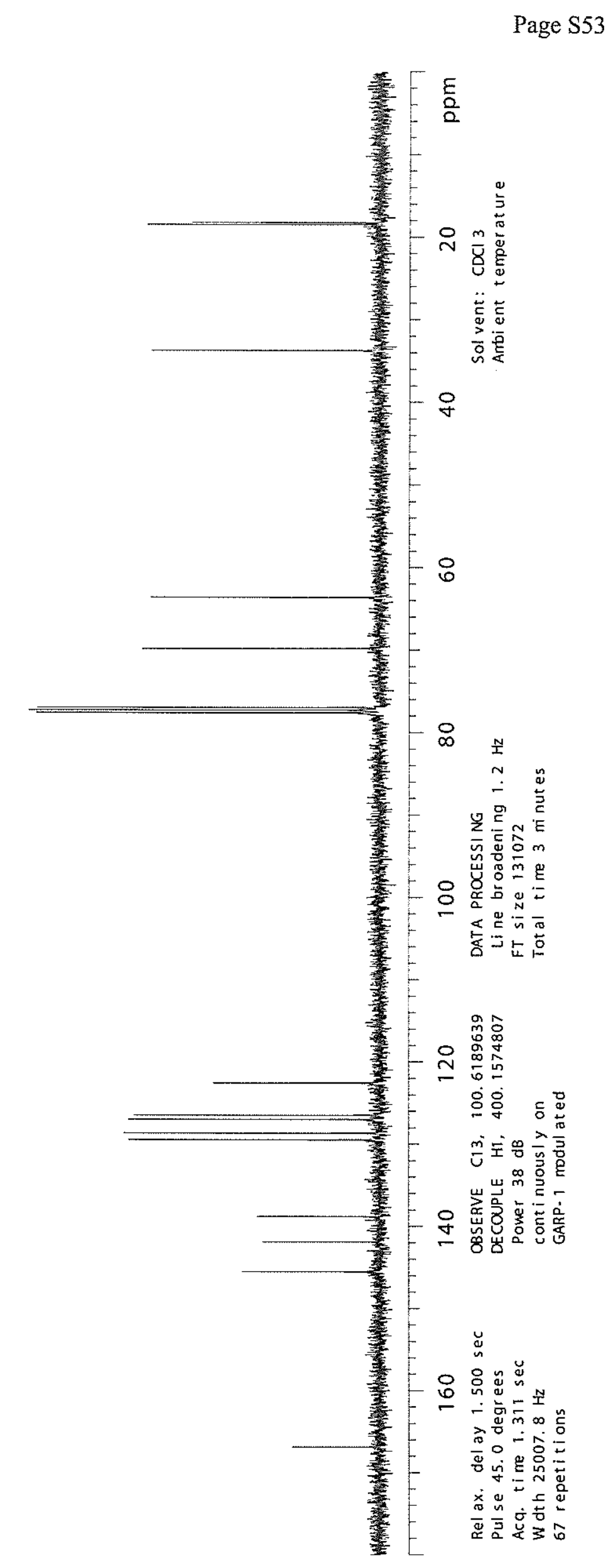




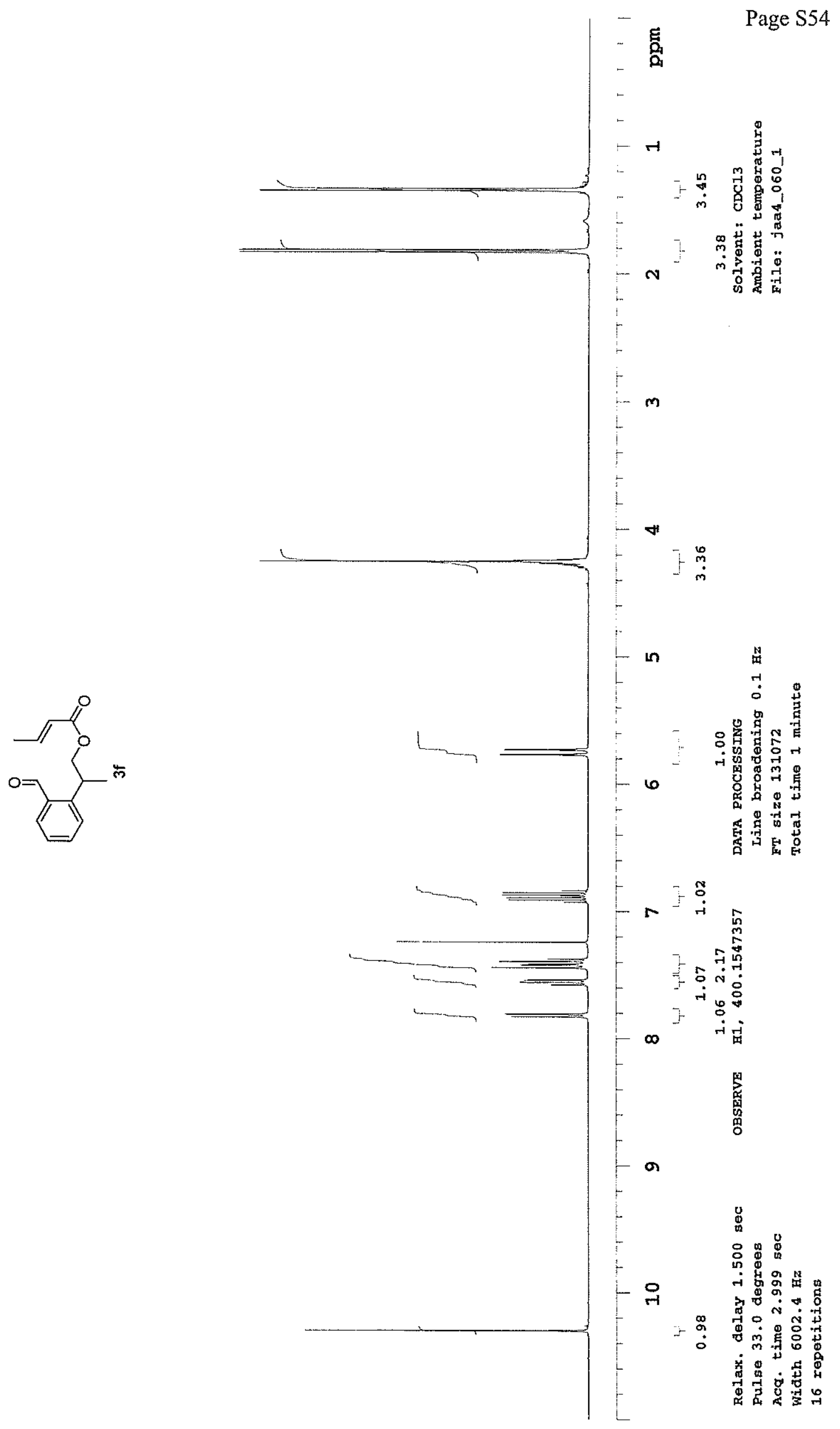




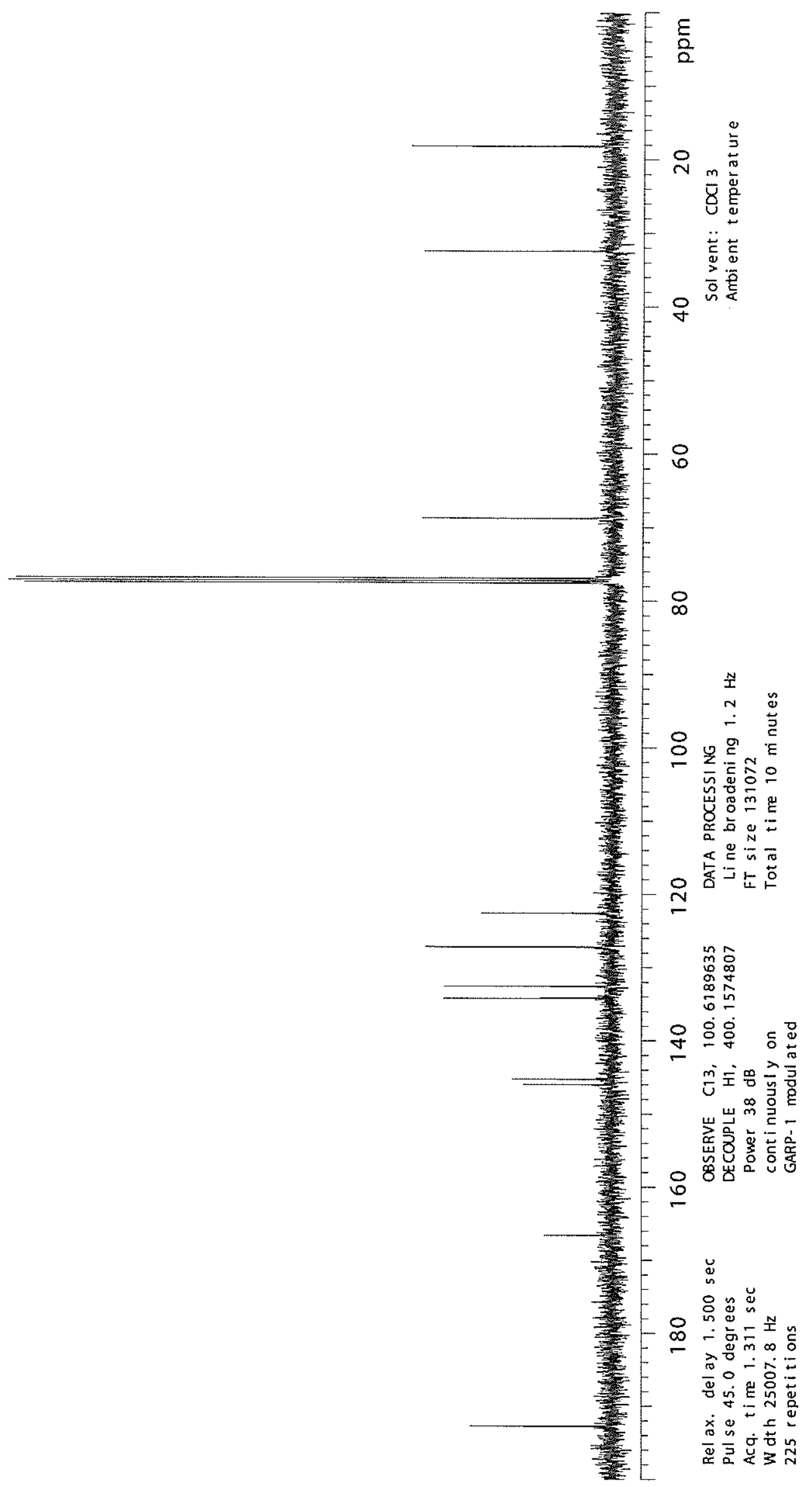




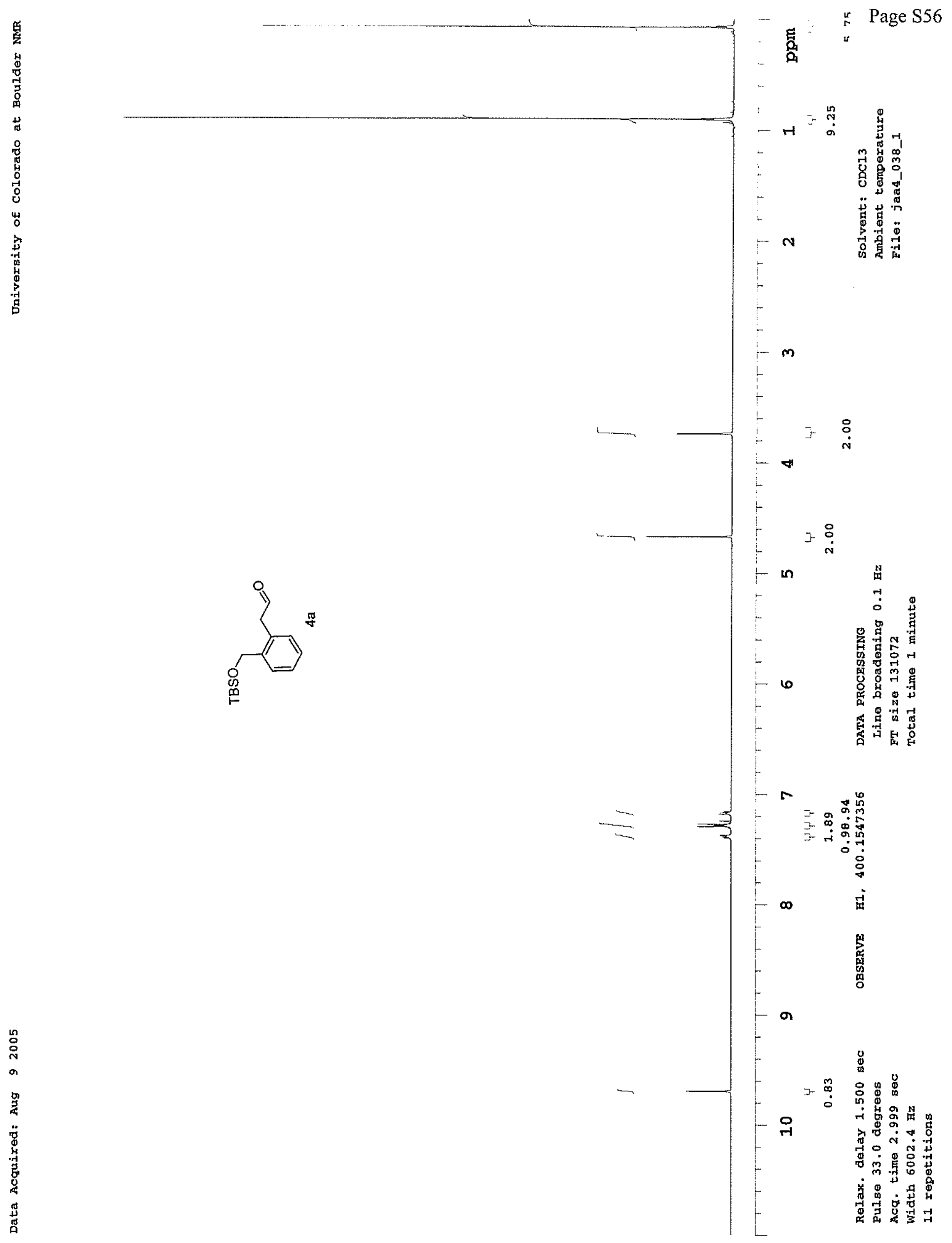




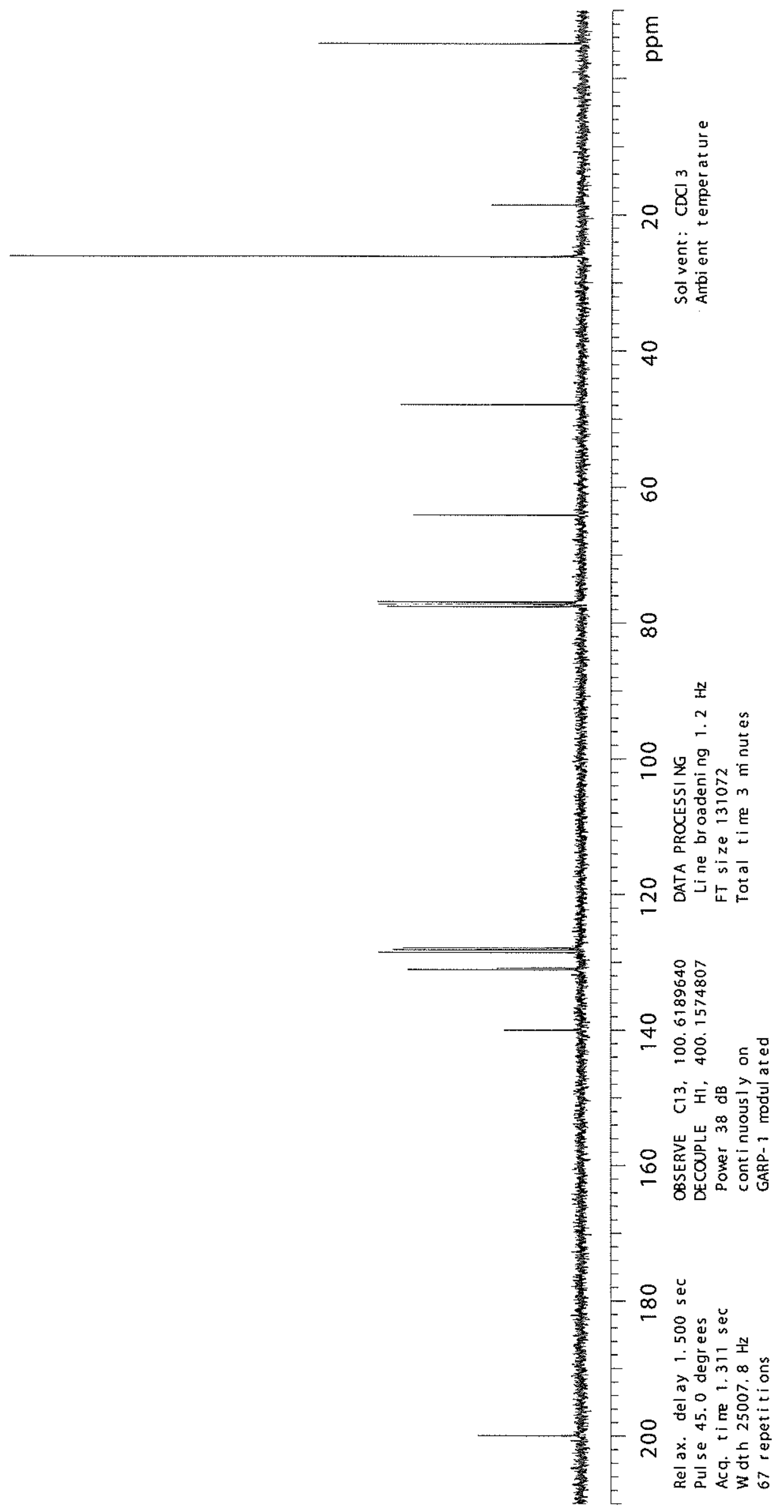




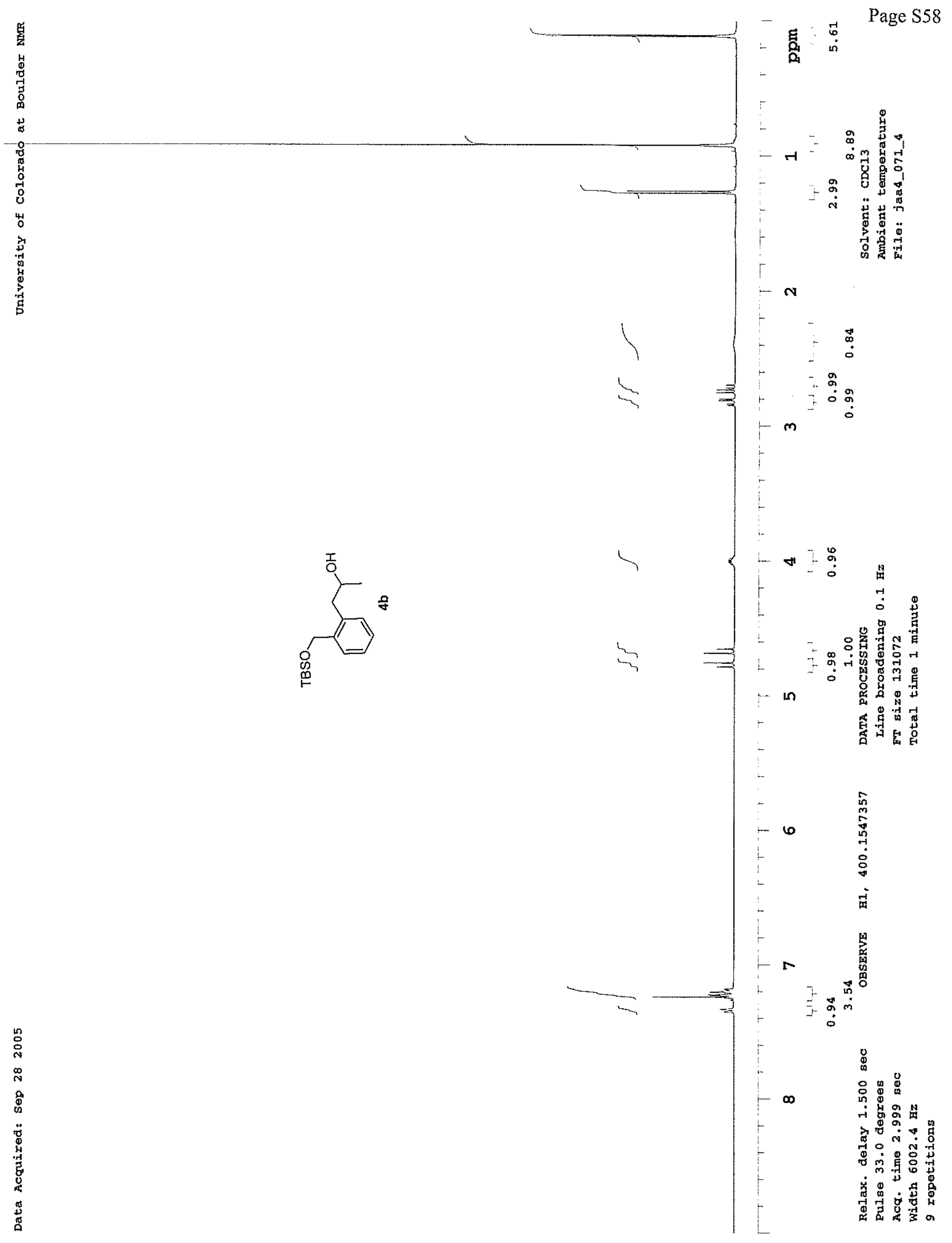



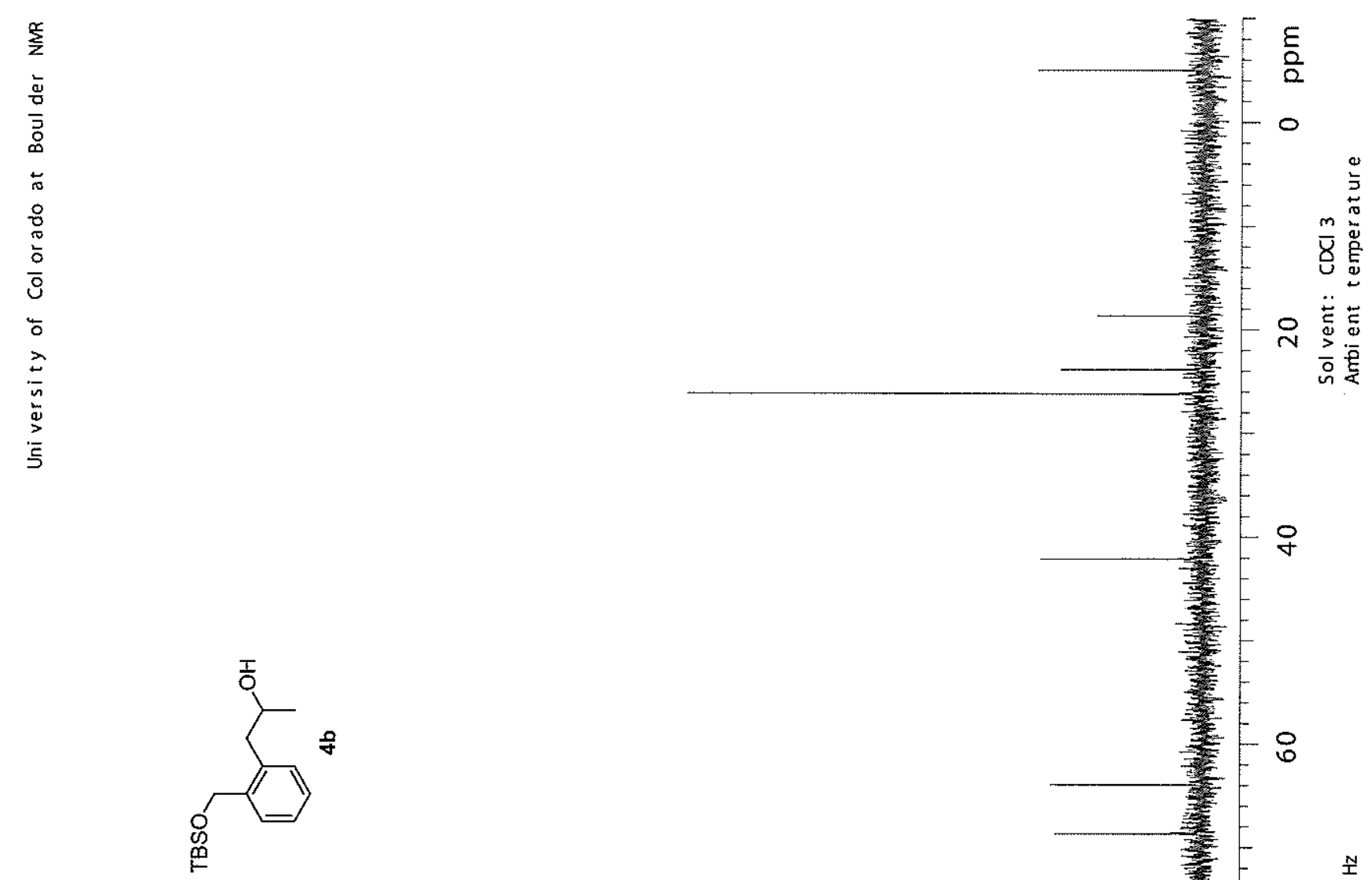


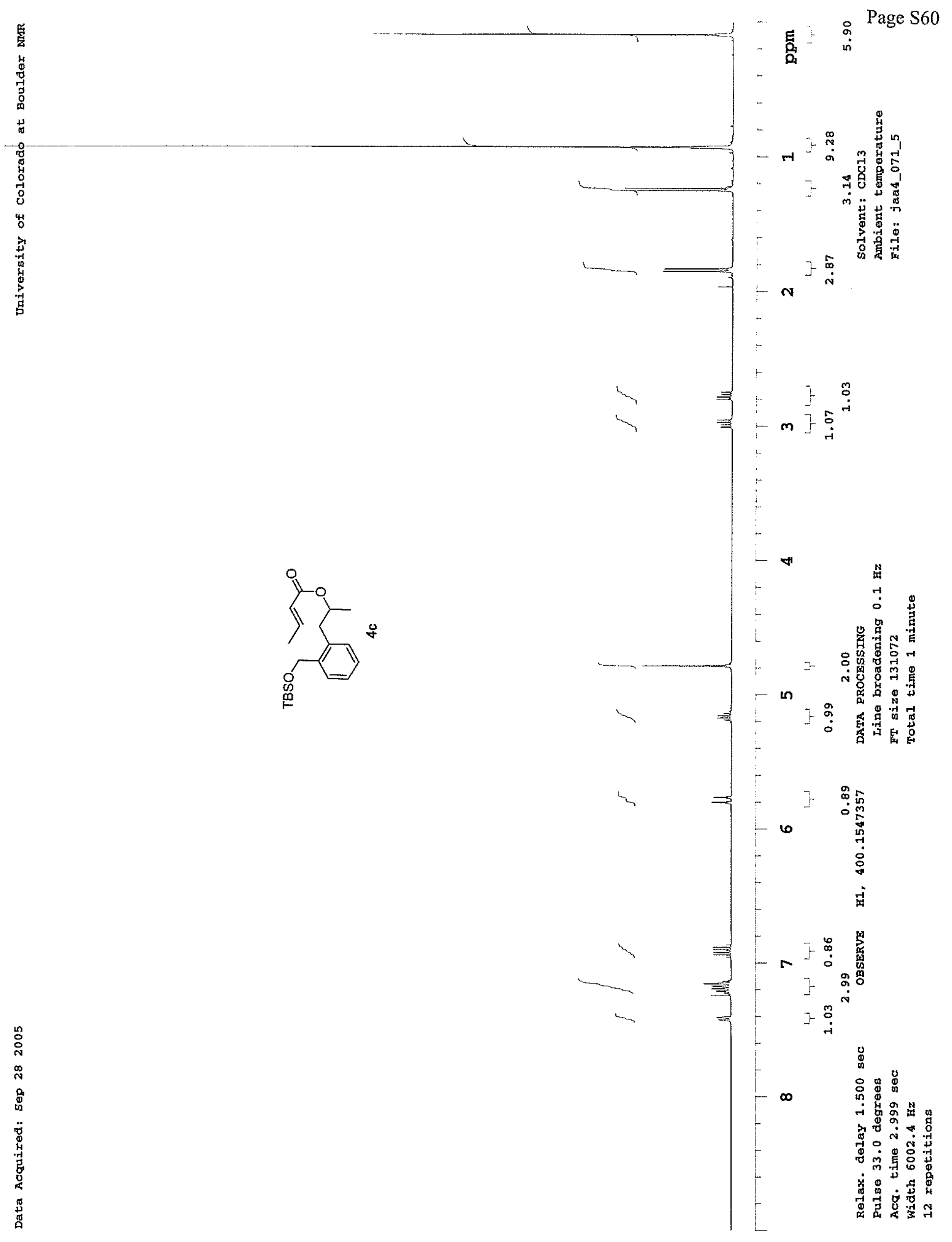




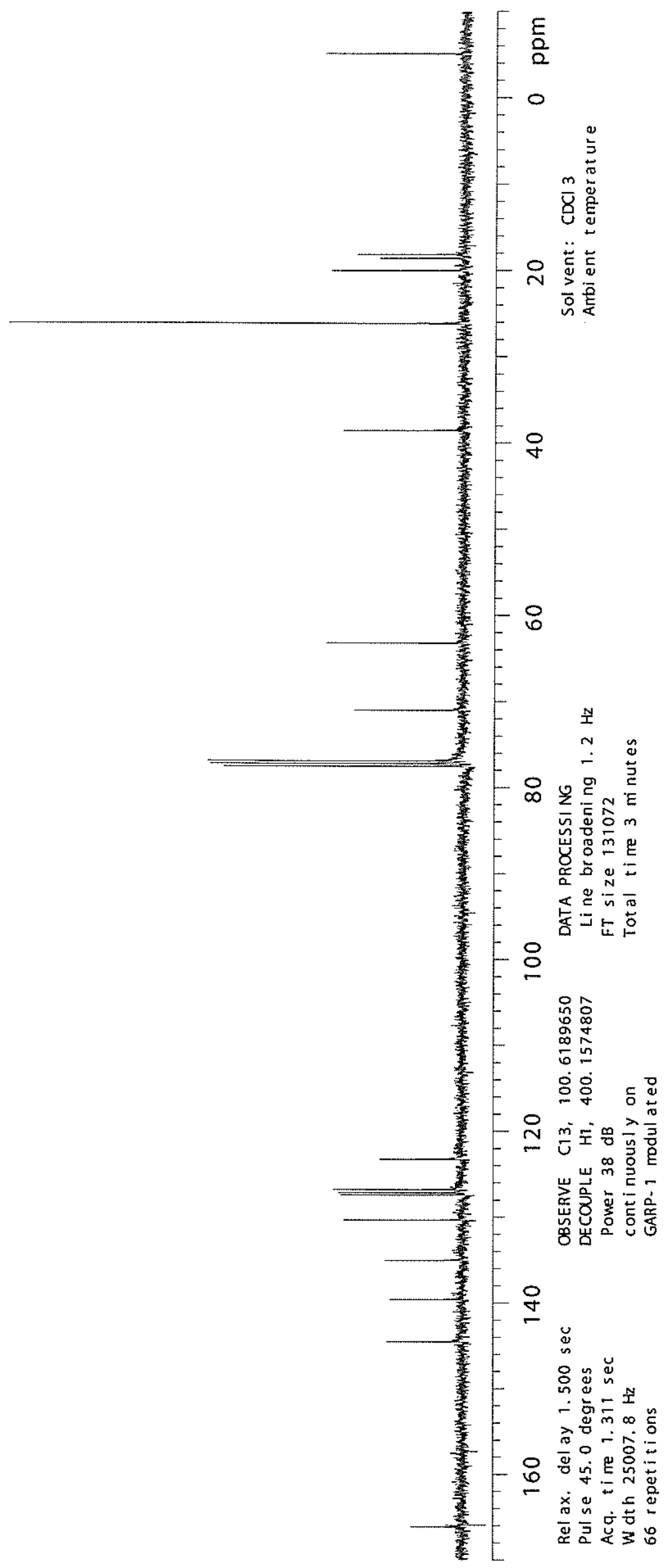




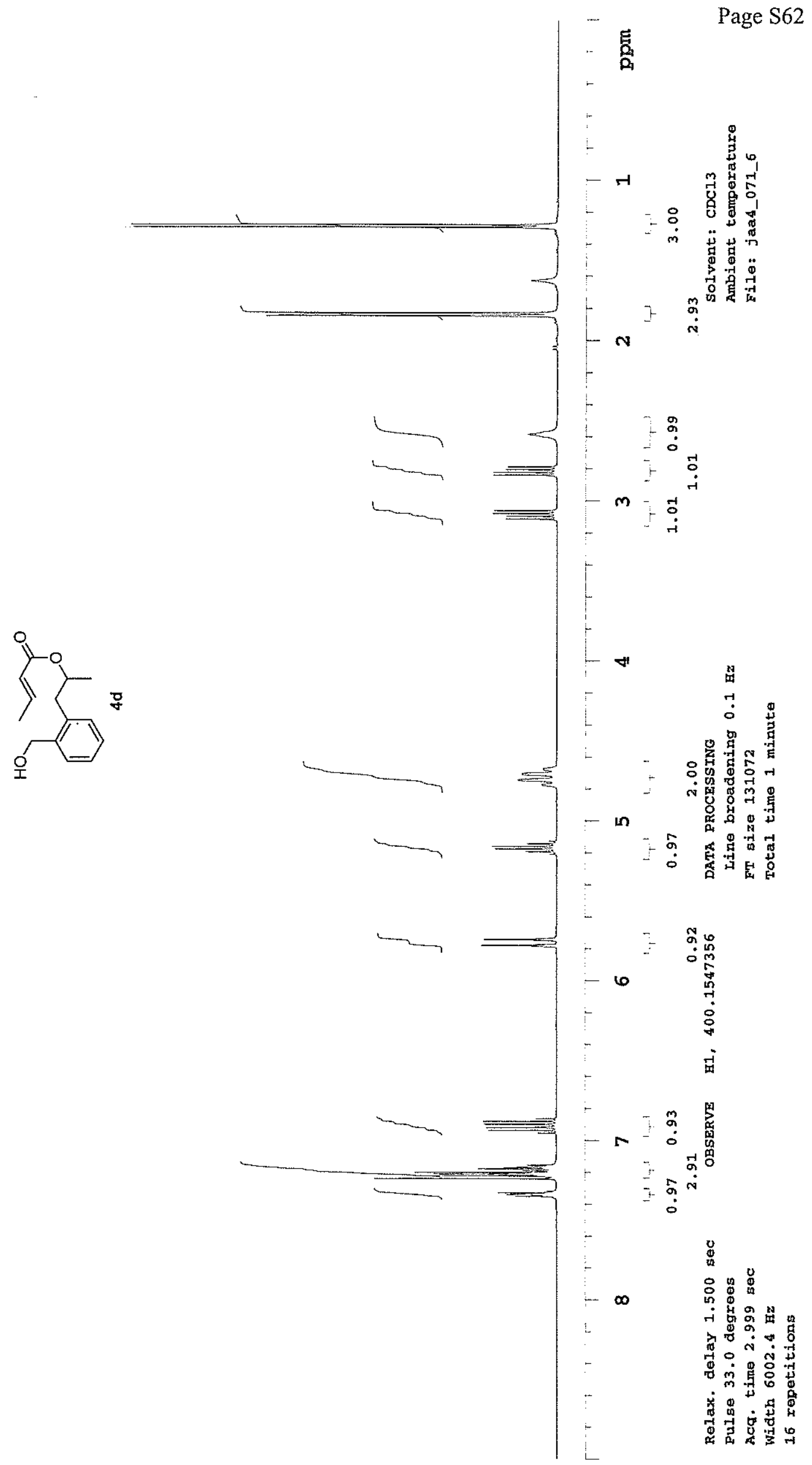




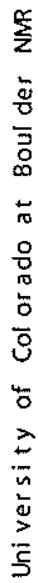

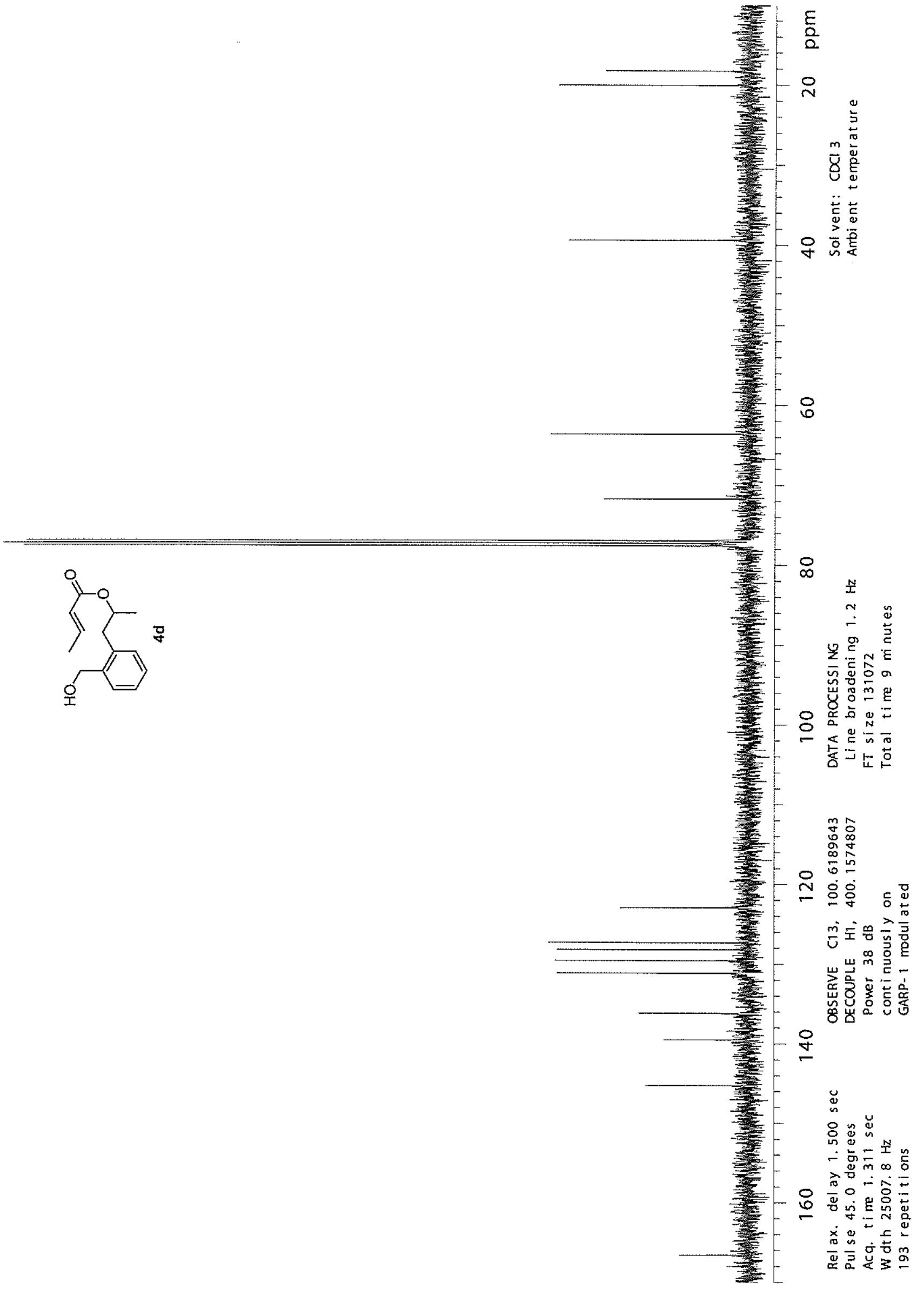




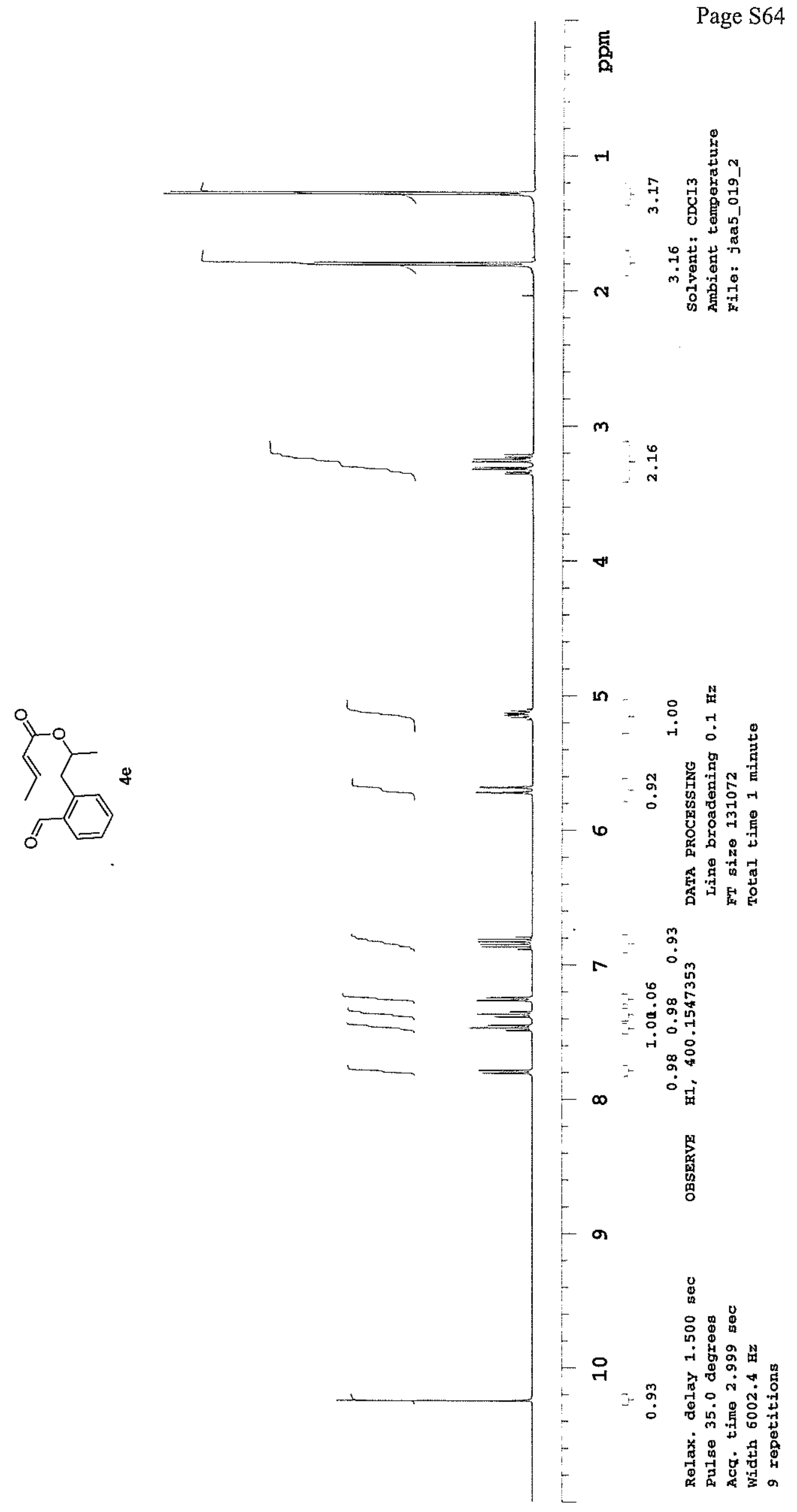




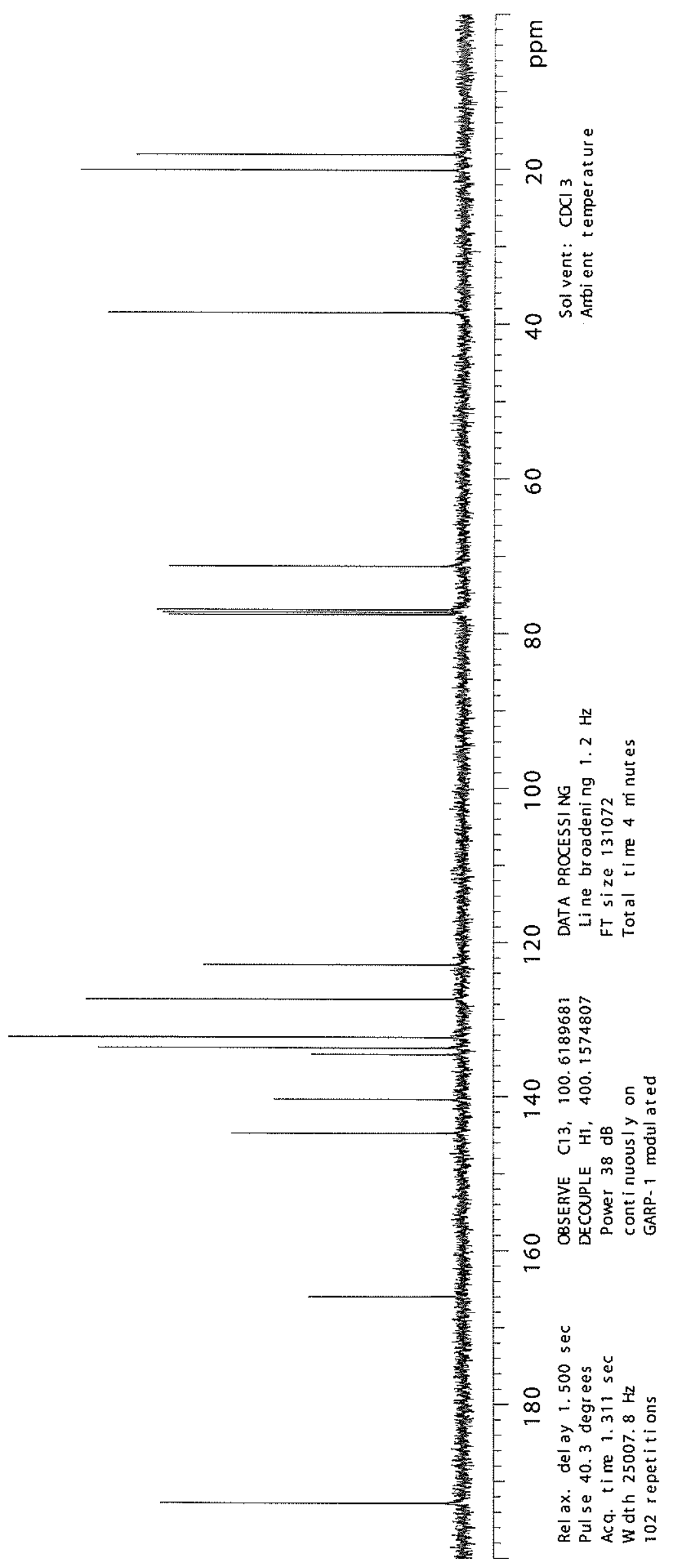




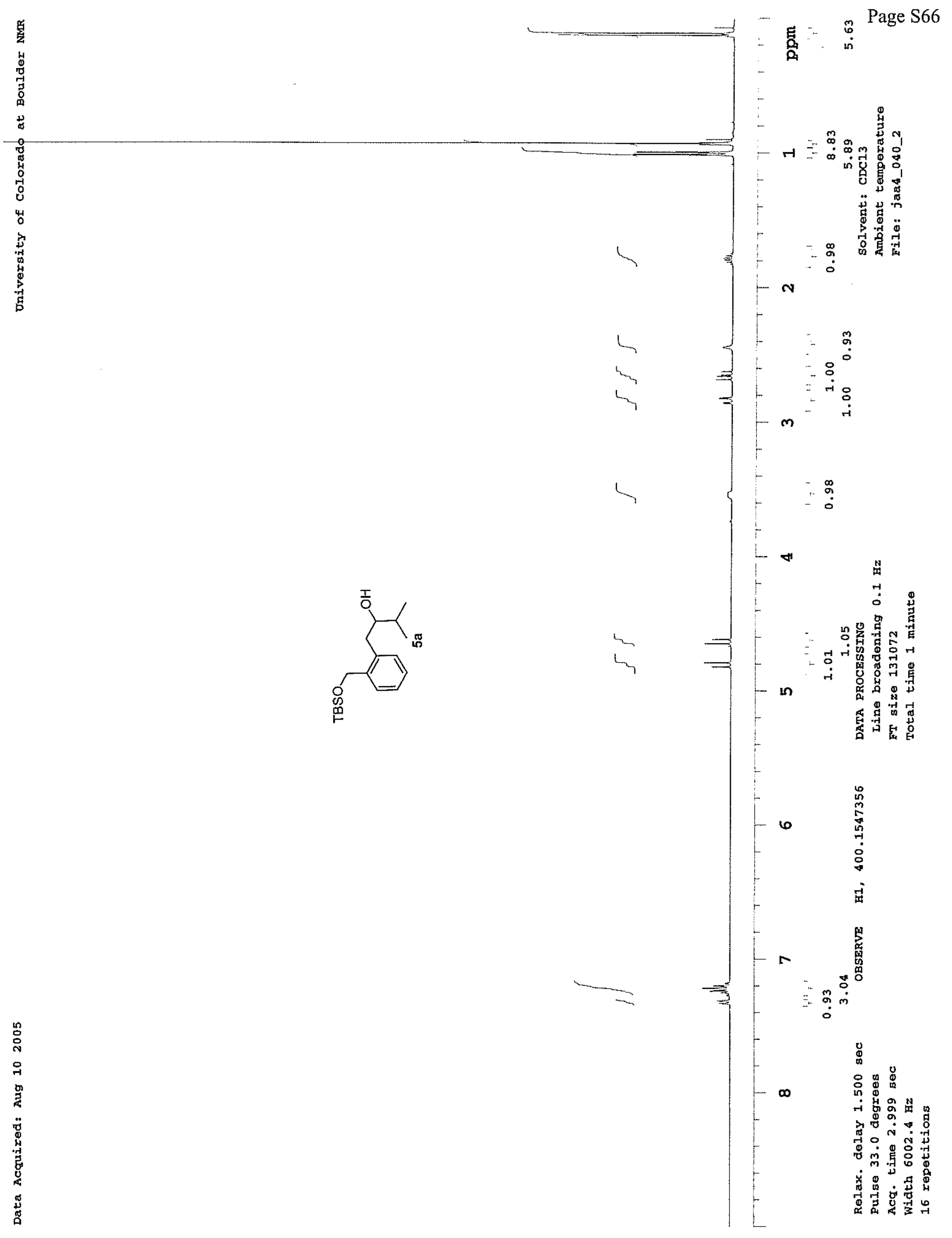


Page S67

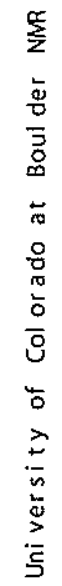

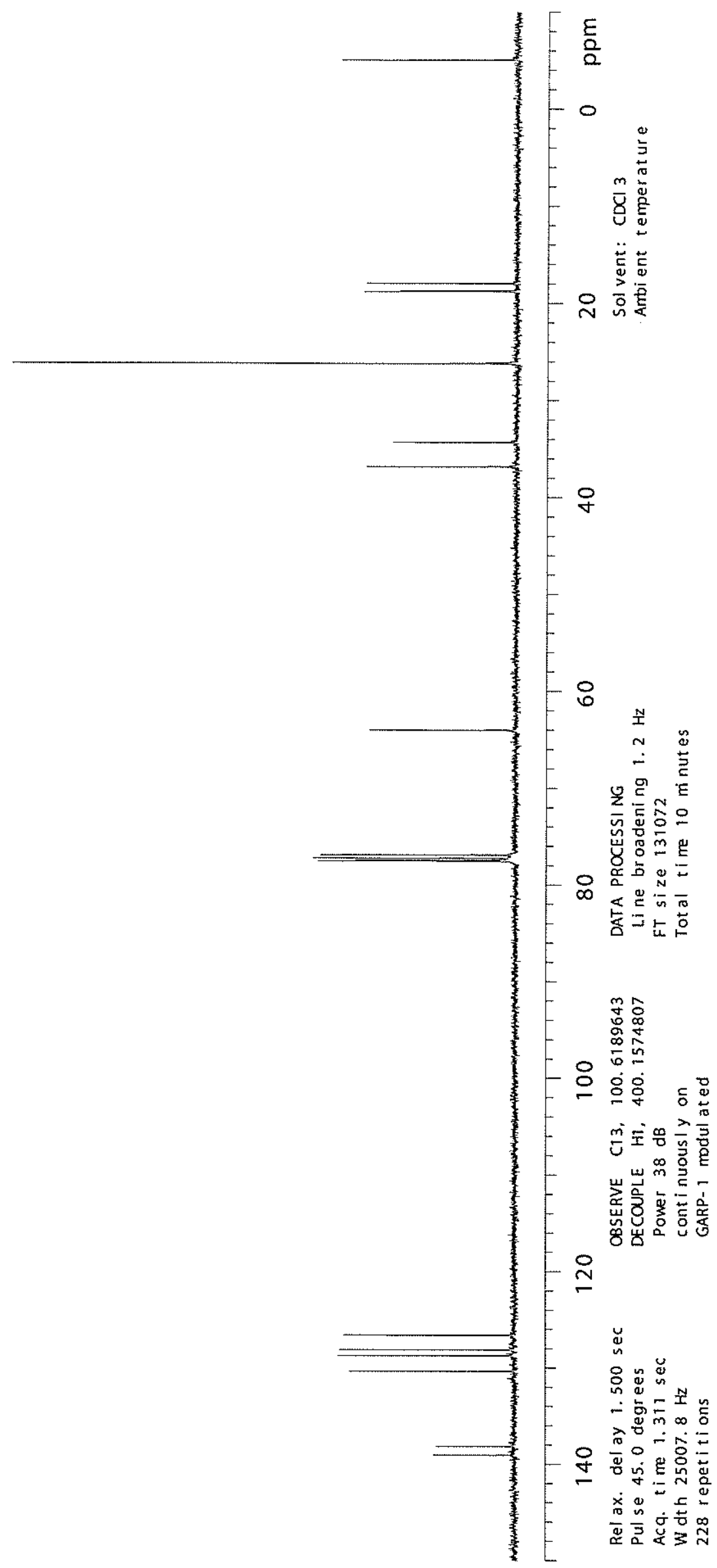




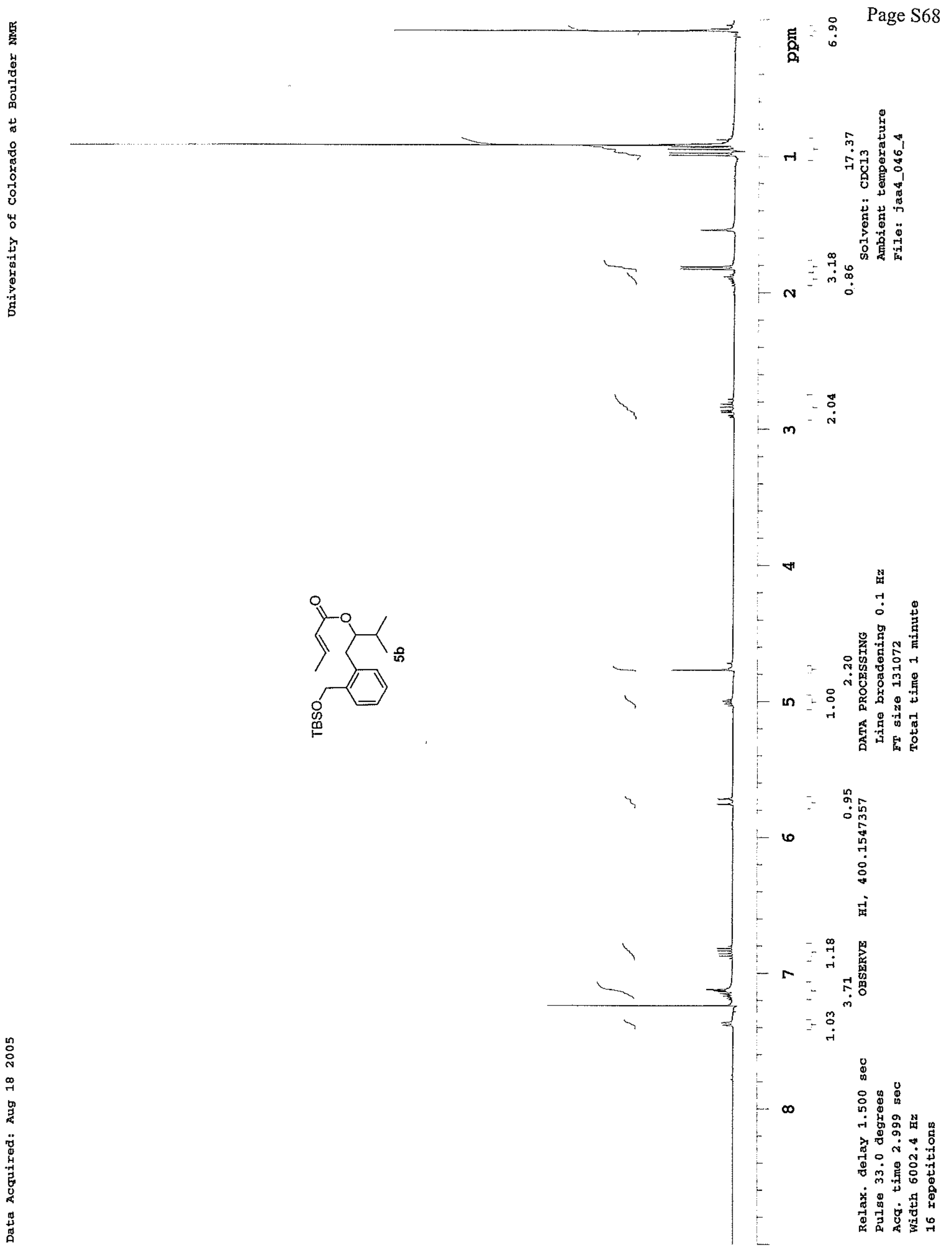




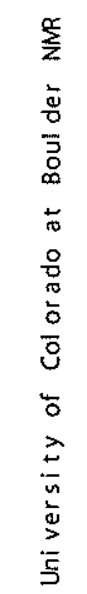

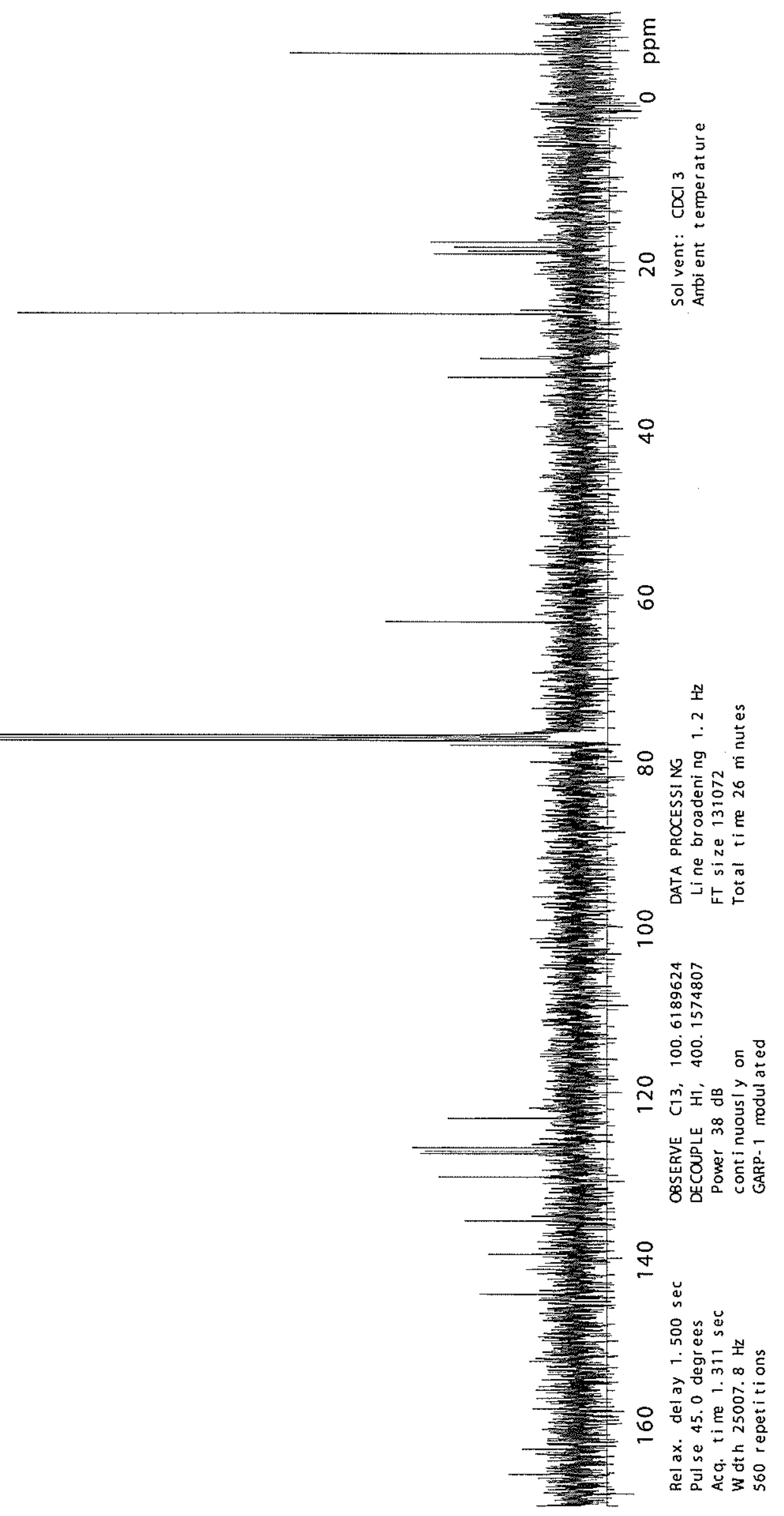



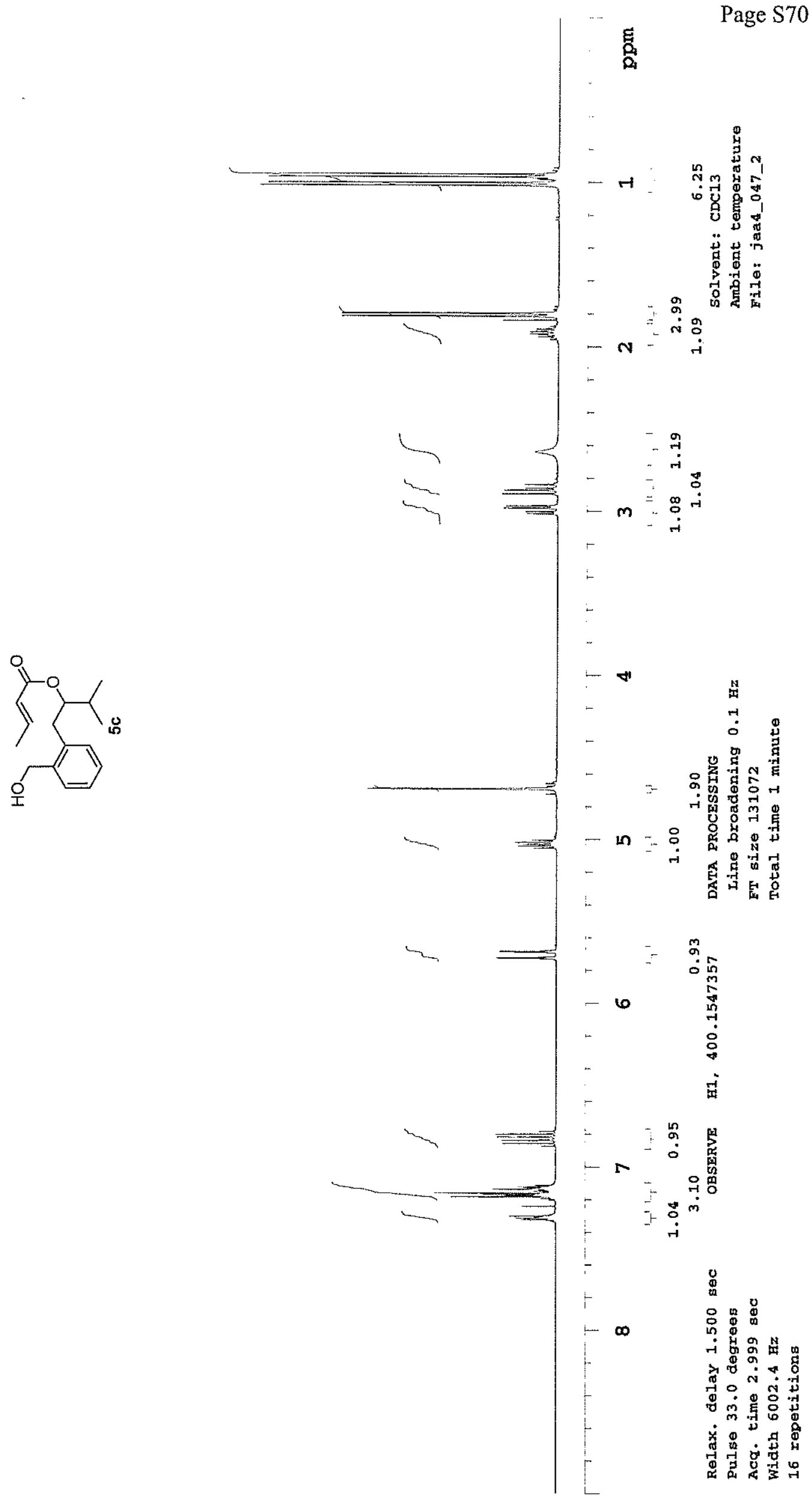


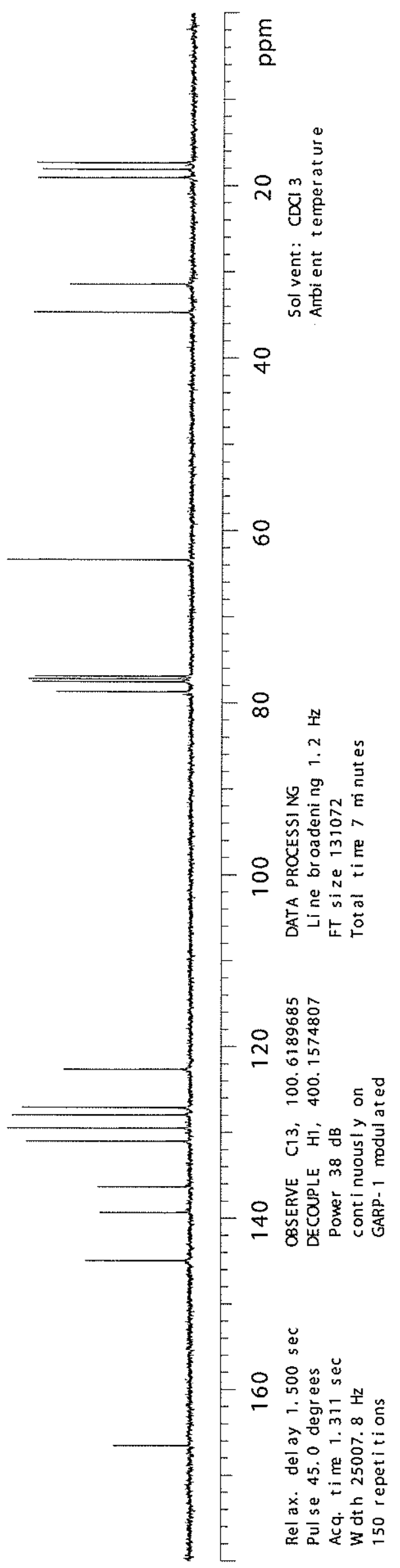




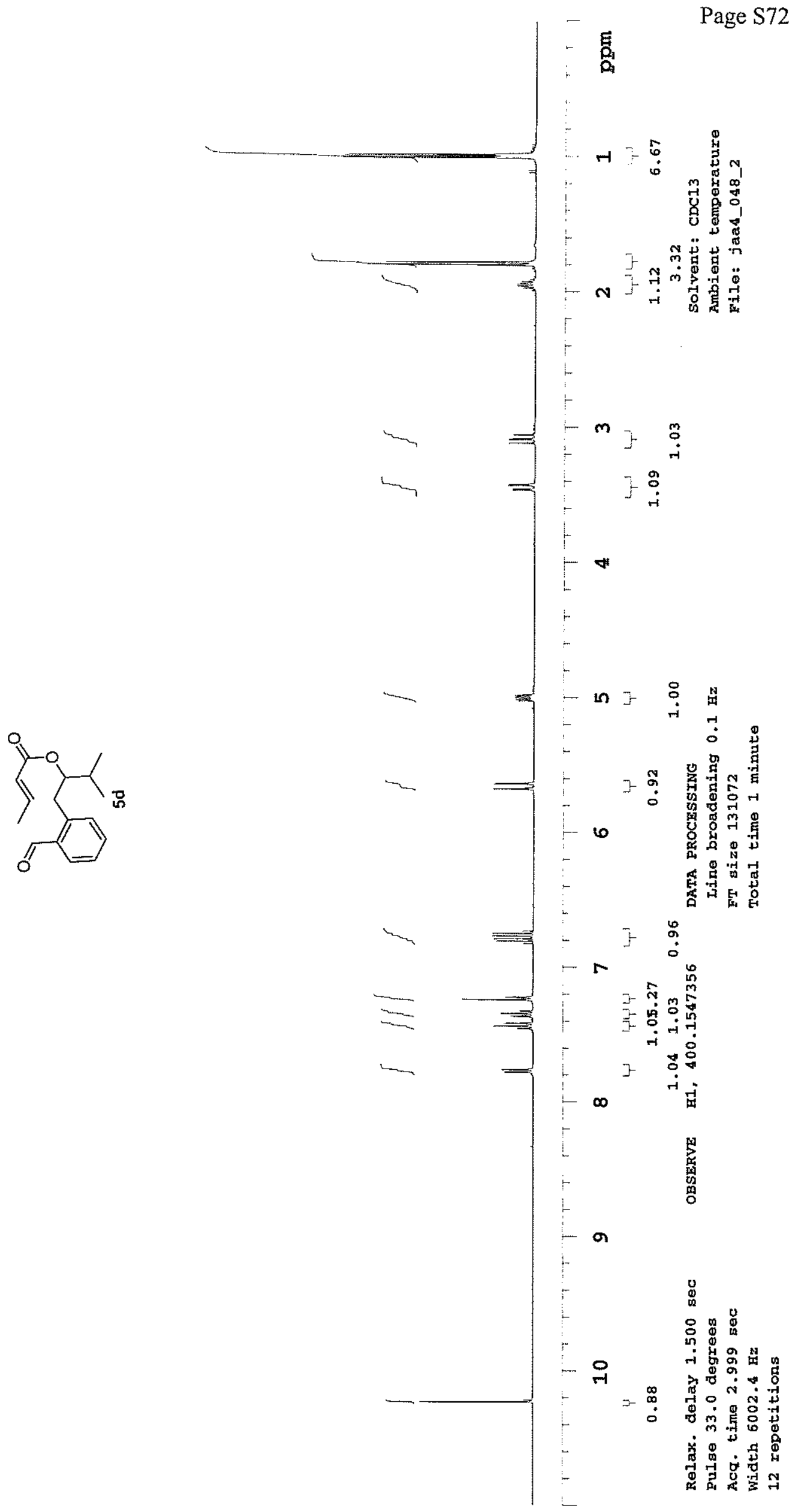




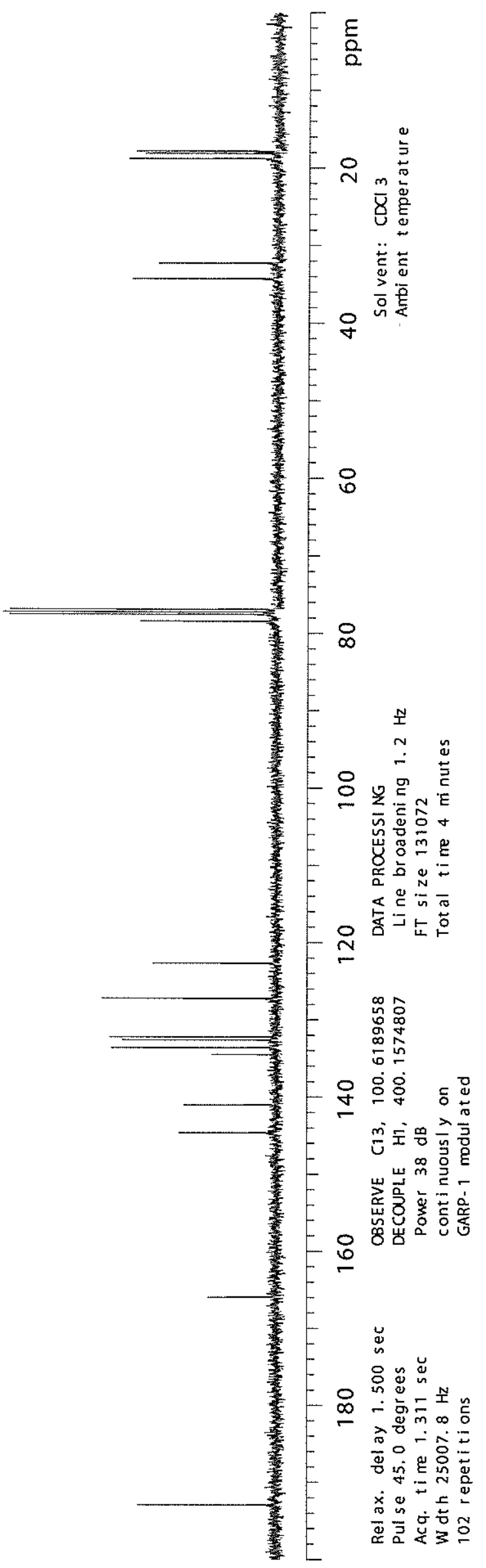




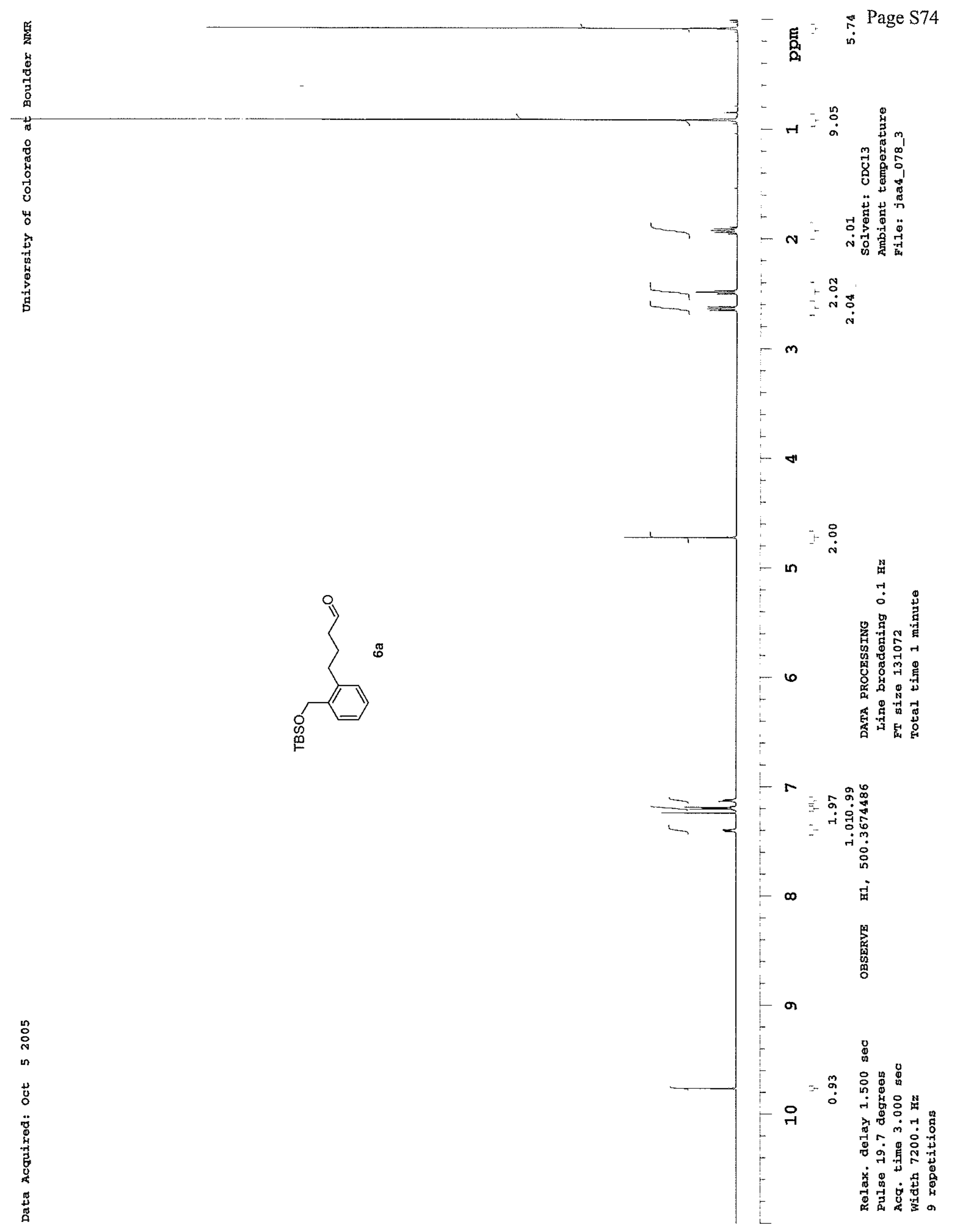




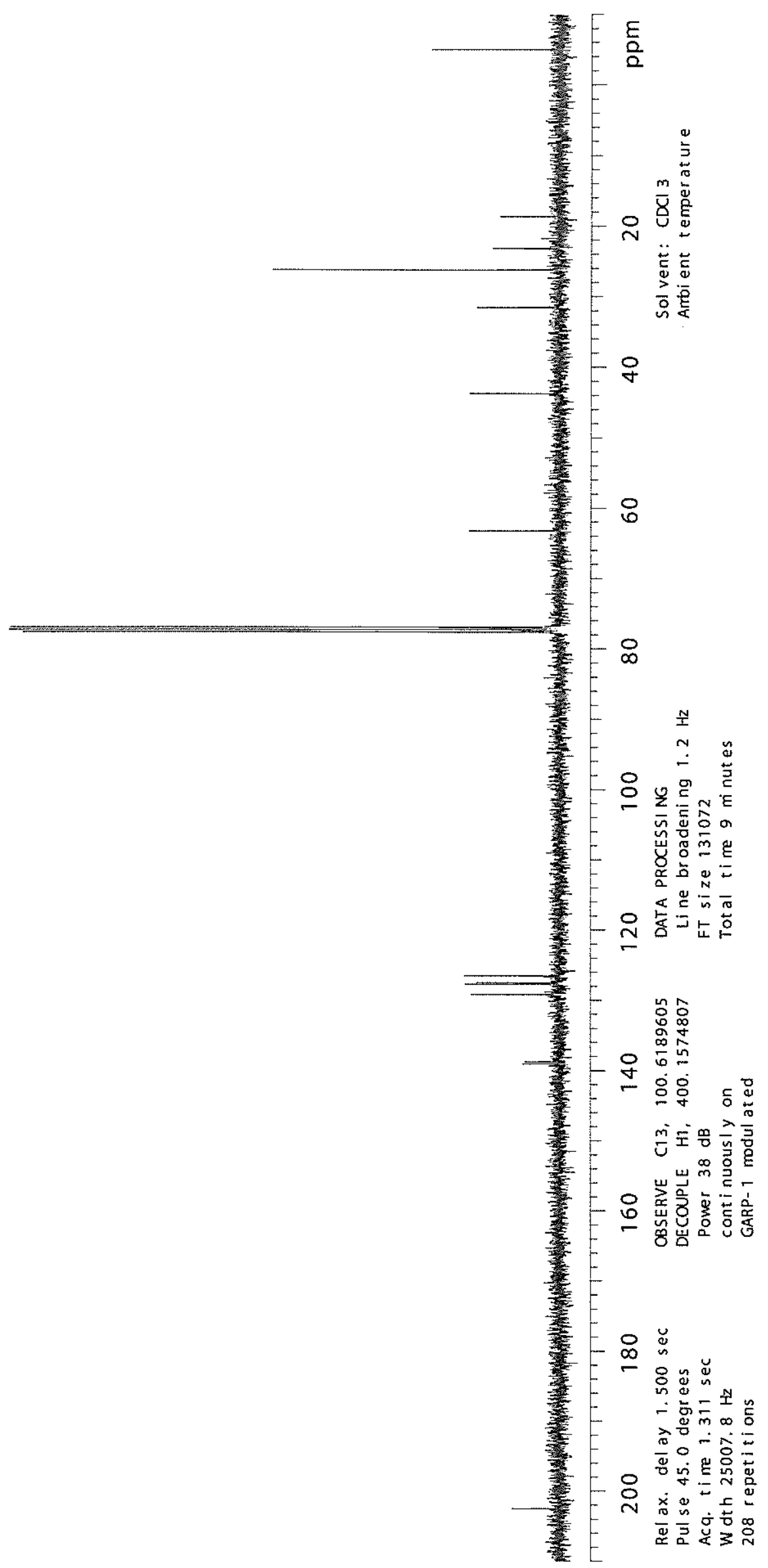




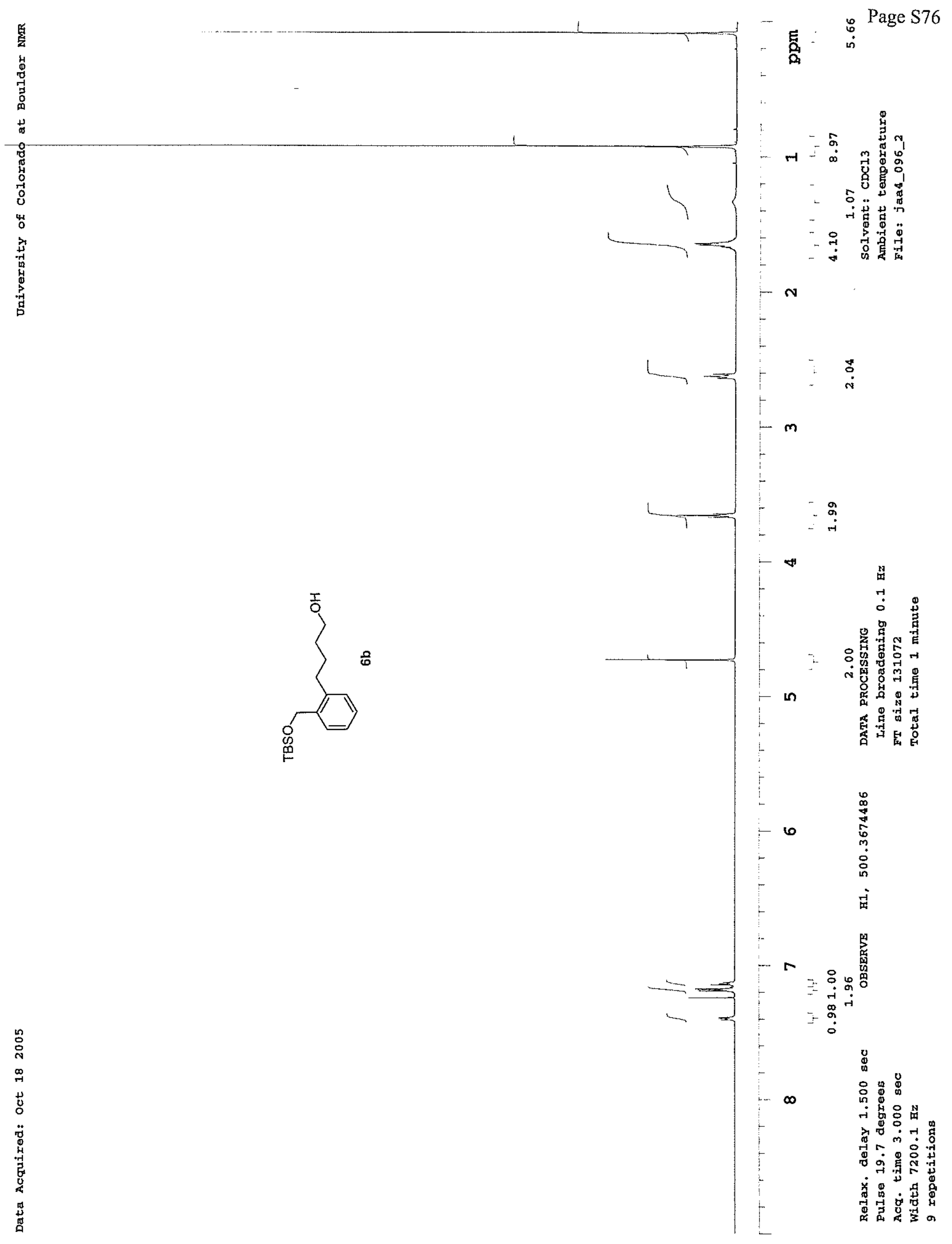




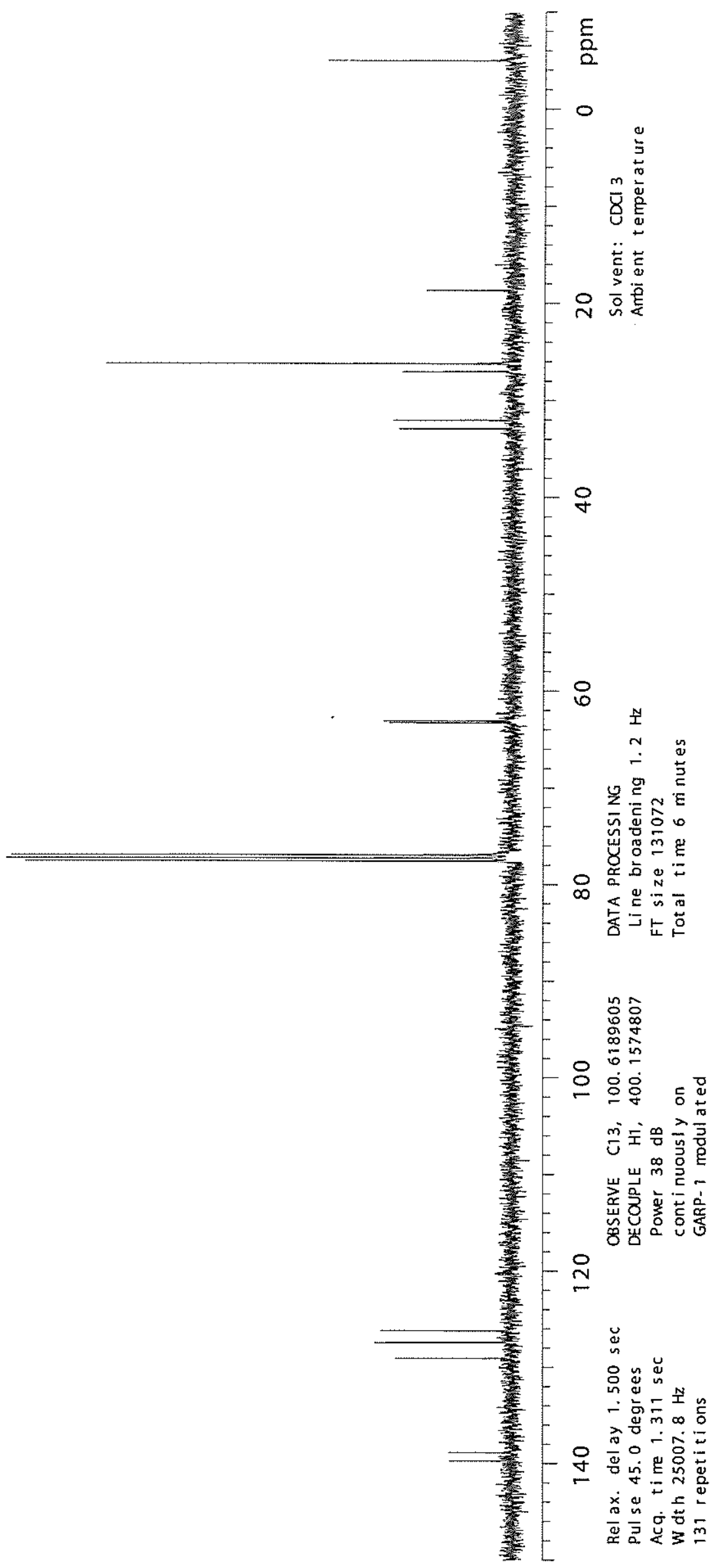




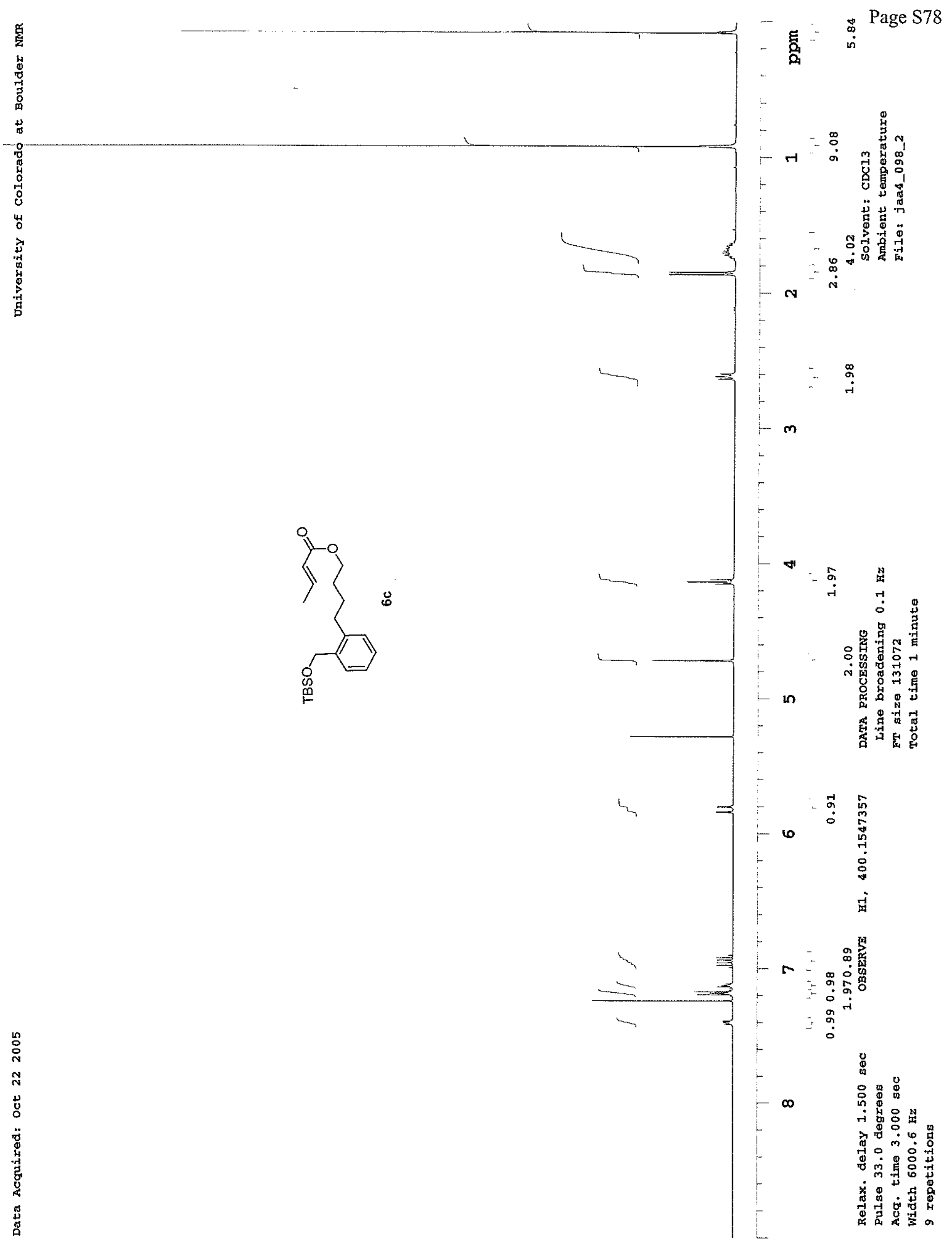



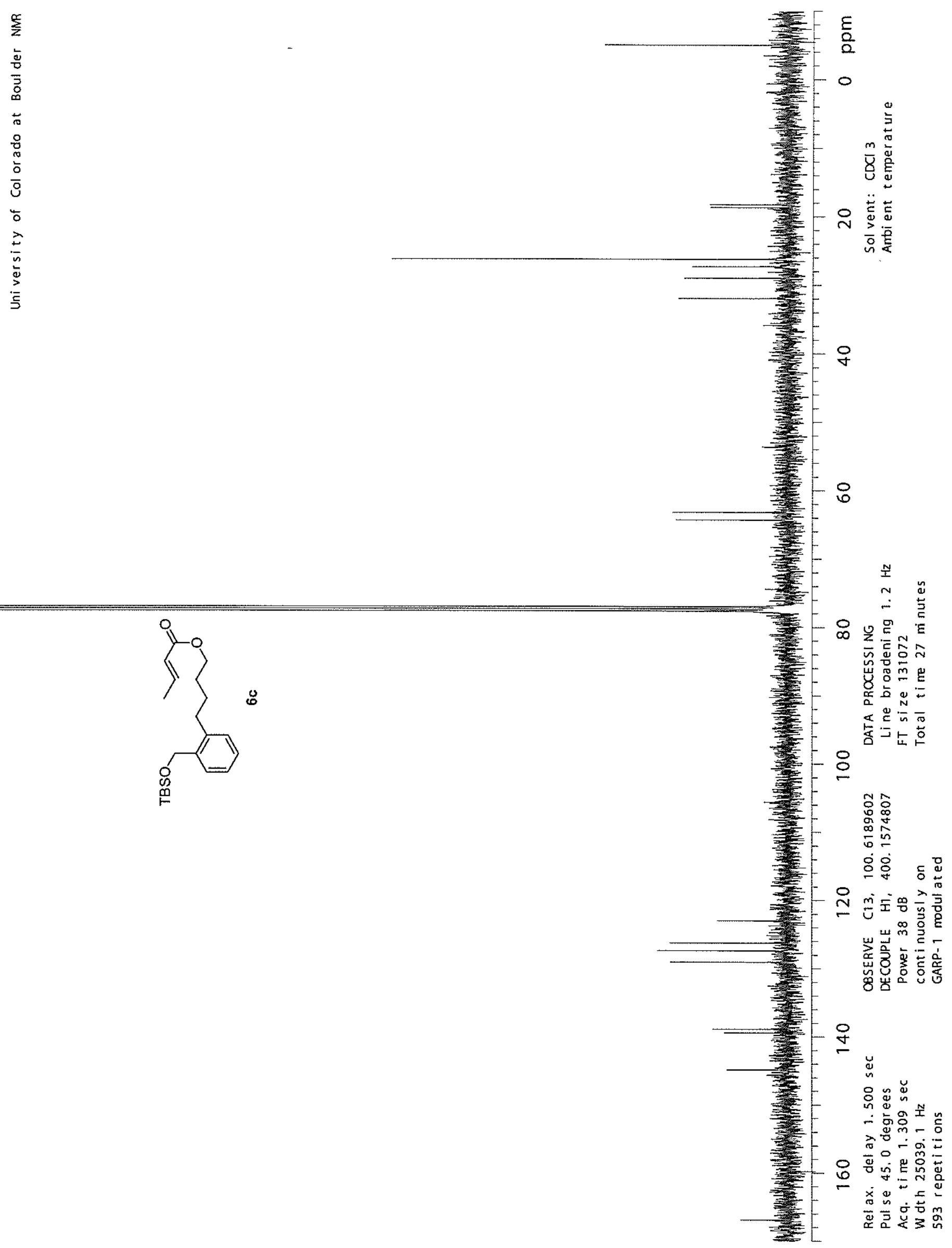
$\mid \begin{aligned} & -\mathbf{g}_{0} \\ & -\end{aligned}$
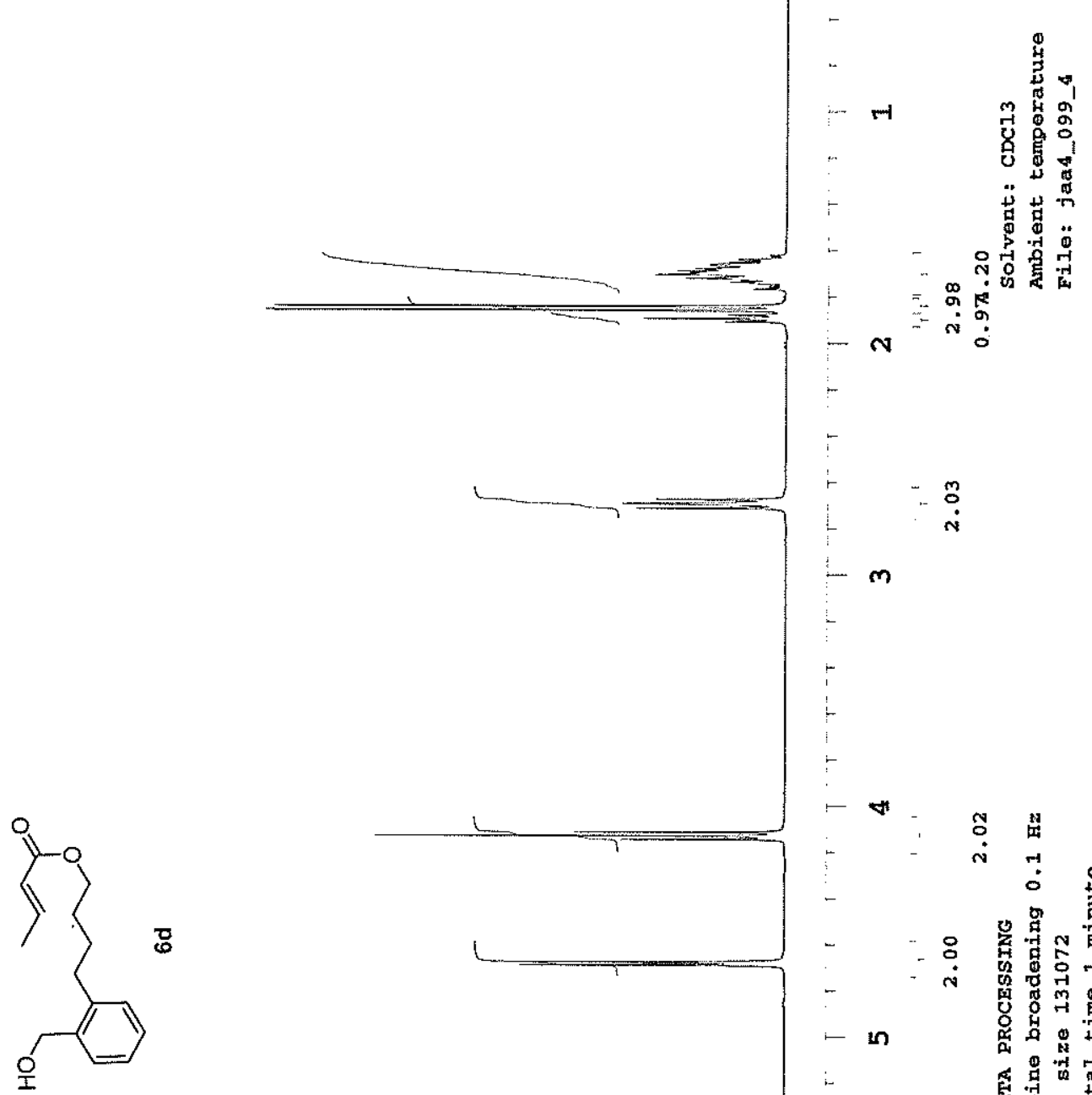

- m

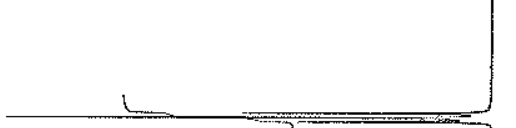

$\vdash$
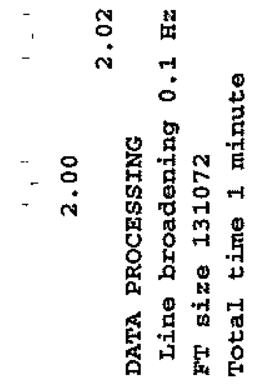

- n

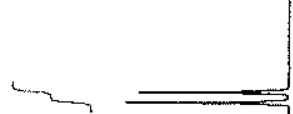

苔是是

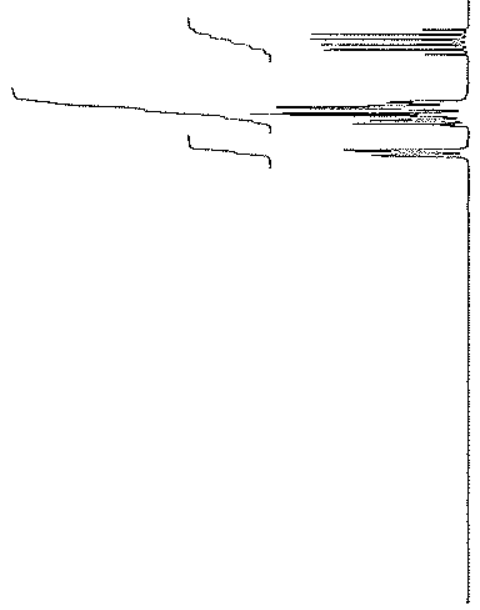

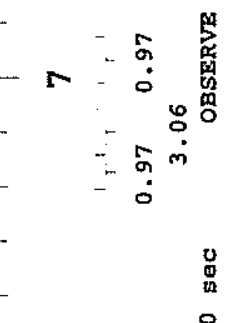

$\infty$

옹

ํㅐ영

我

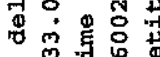

我

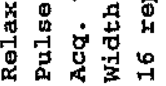


Page S81

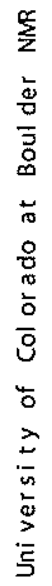

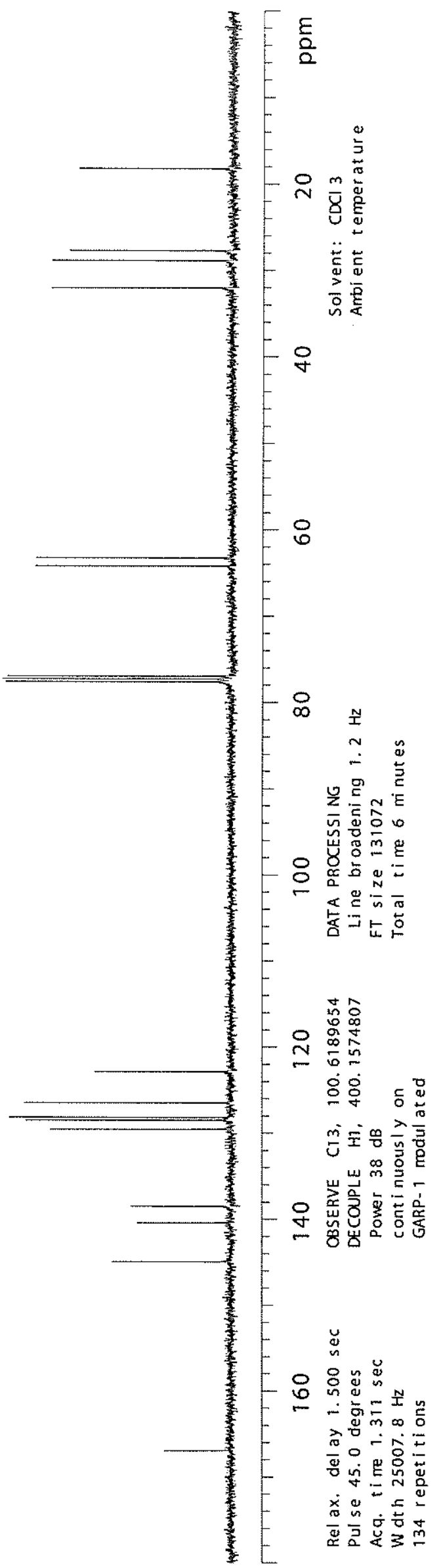




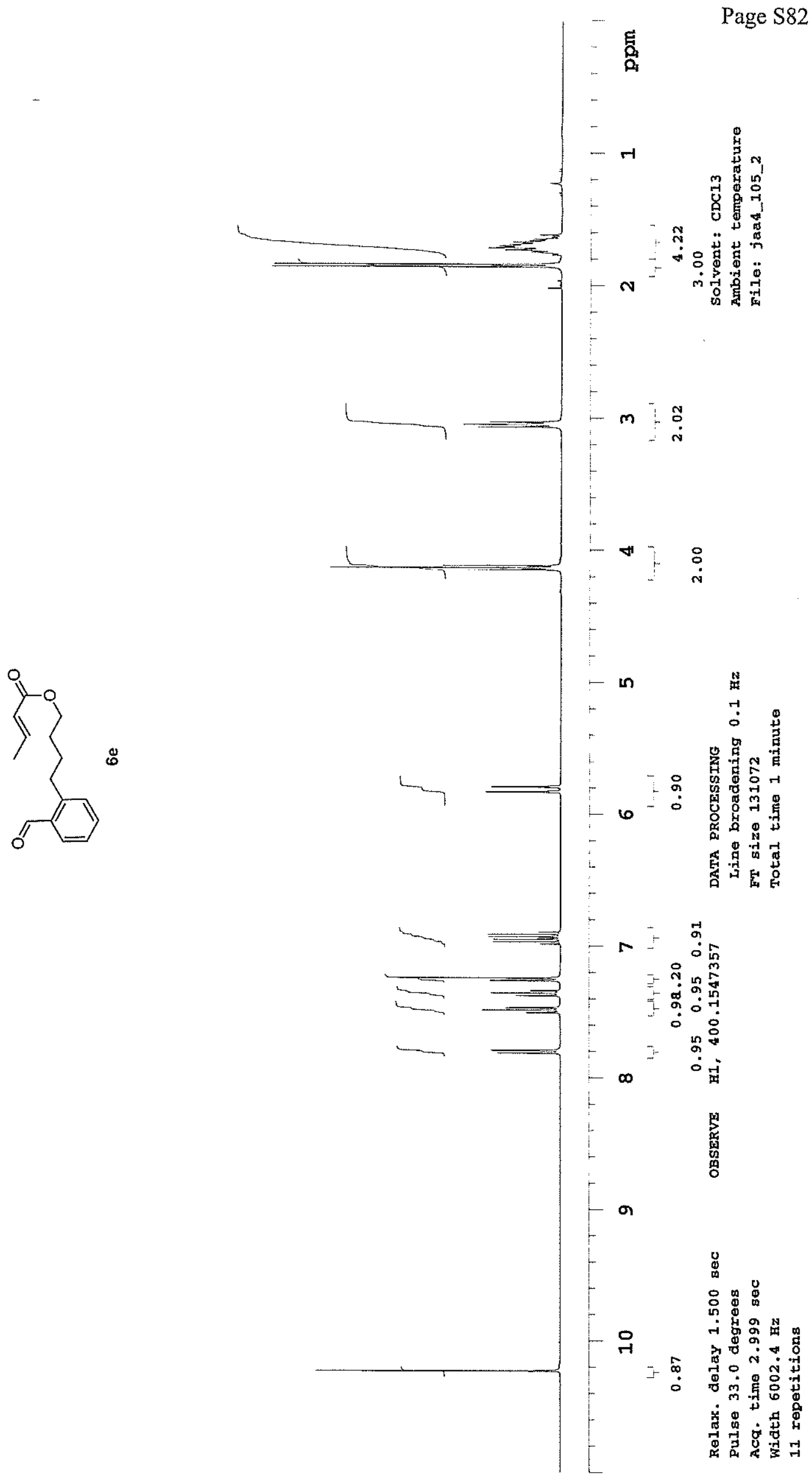




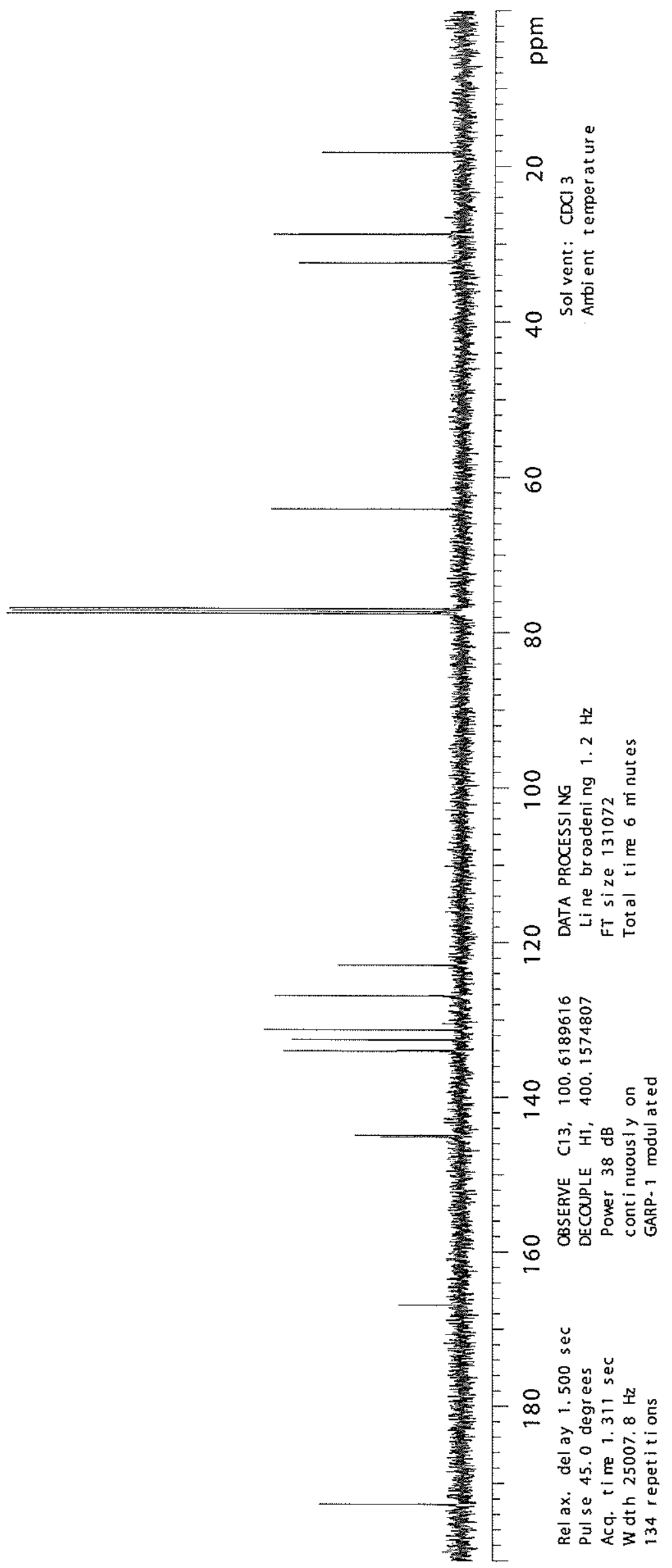




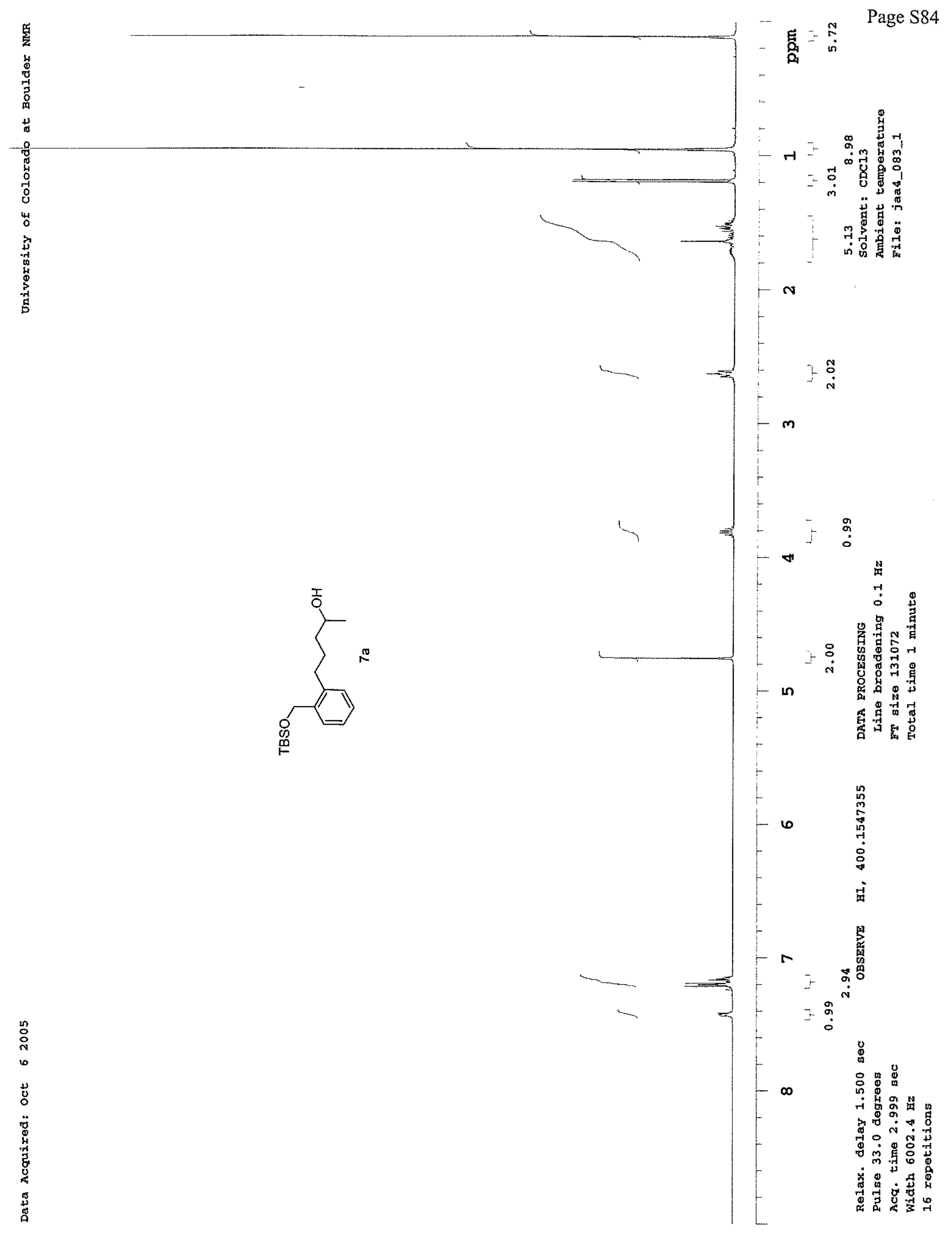


Page S85

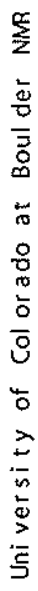
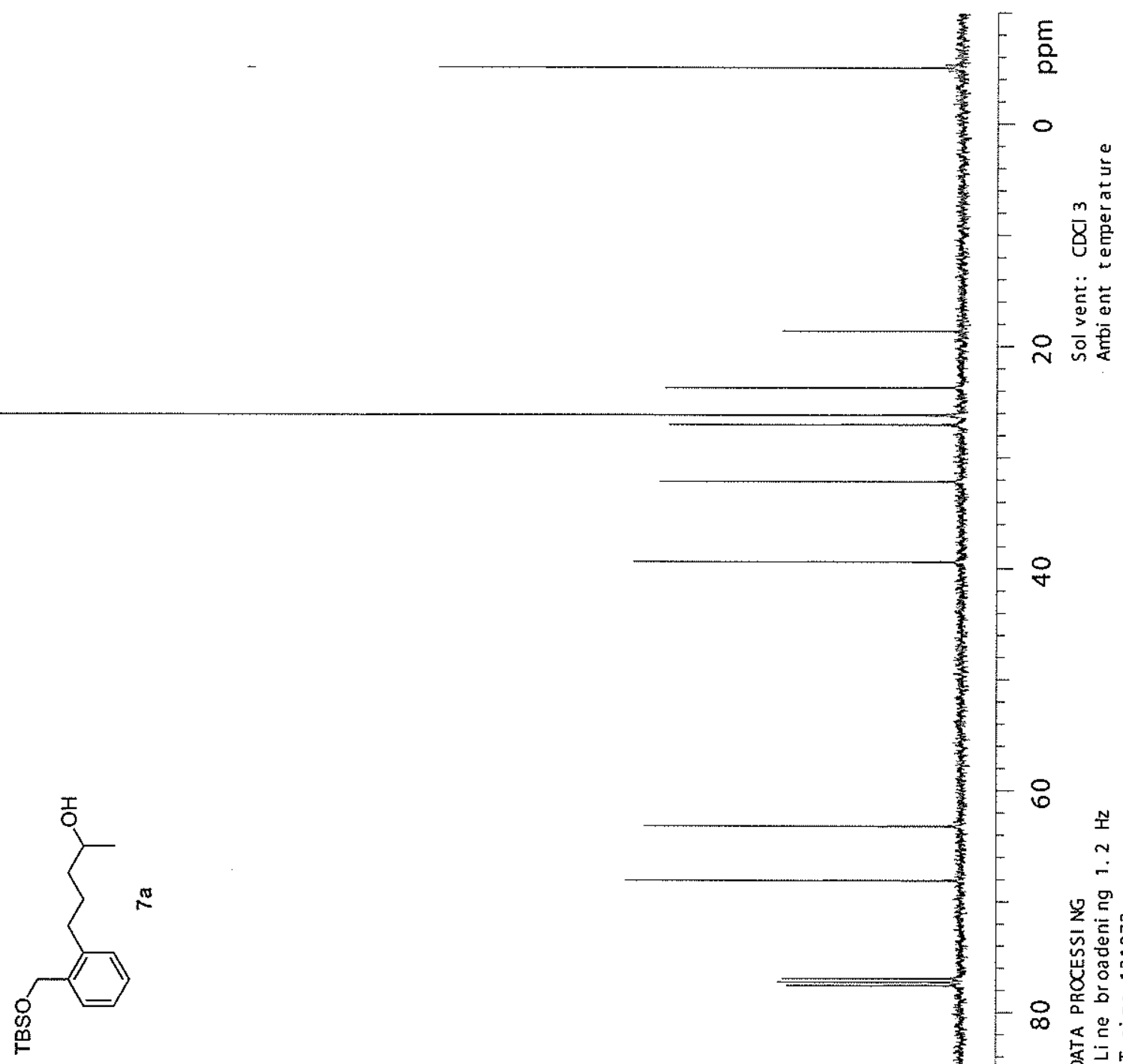

$\sim$ in
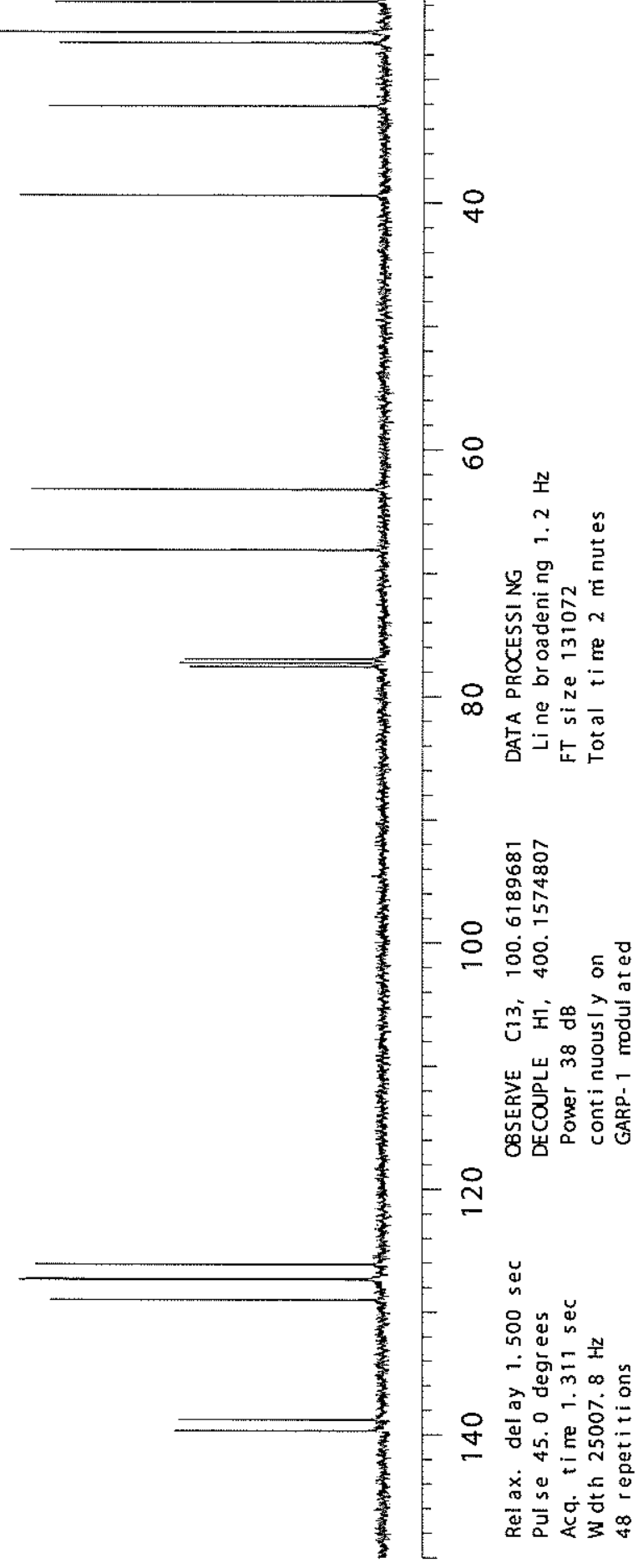


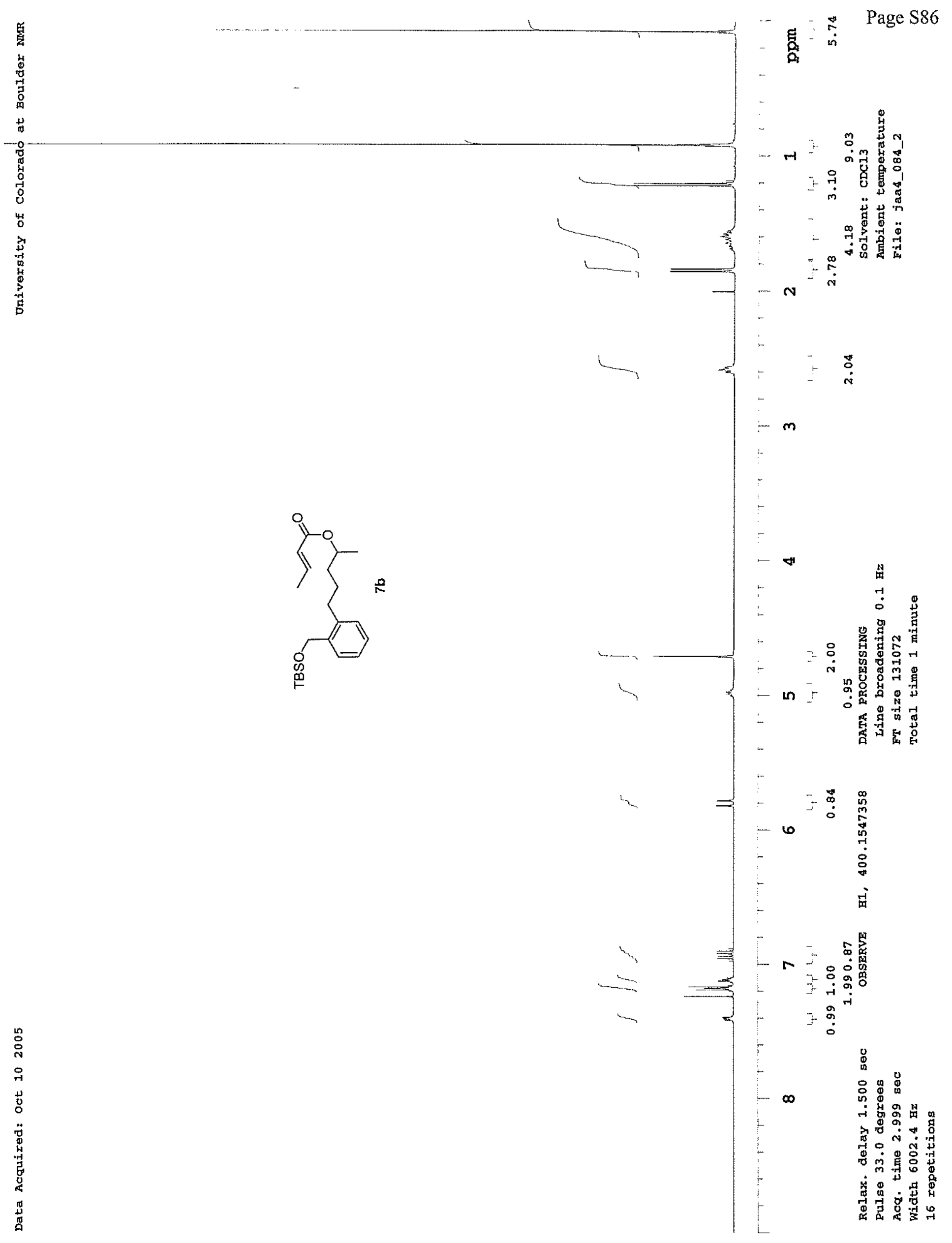




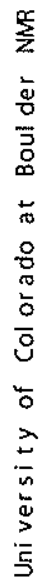

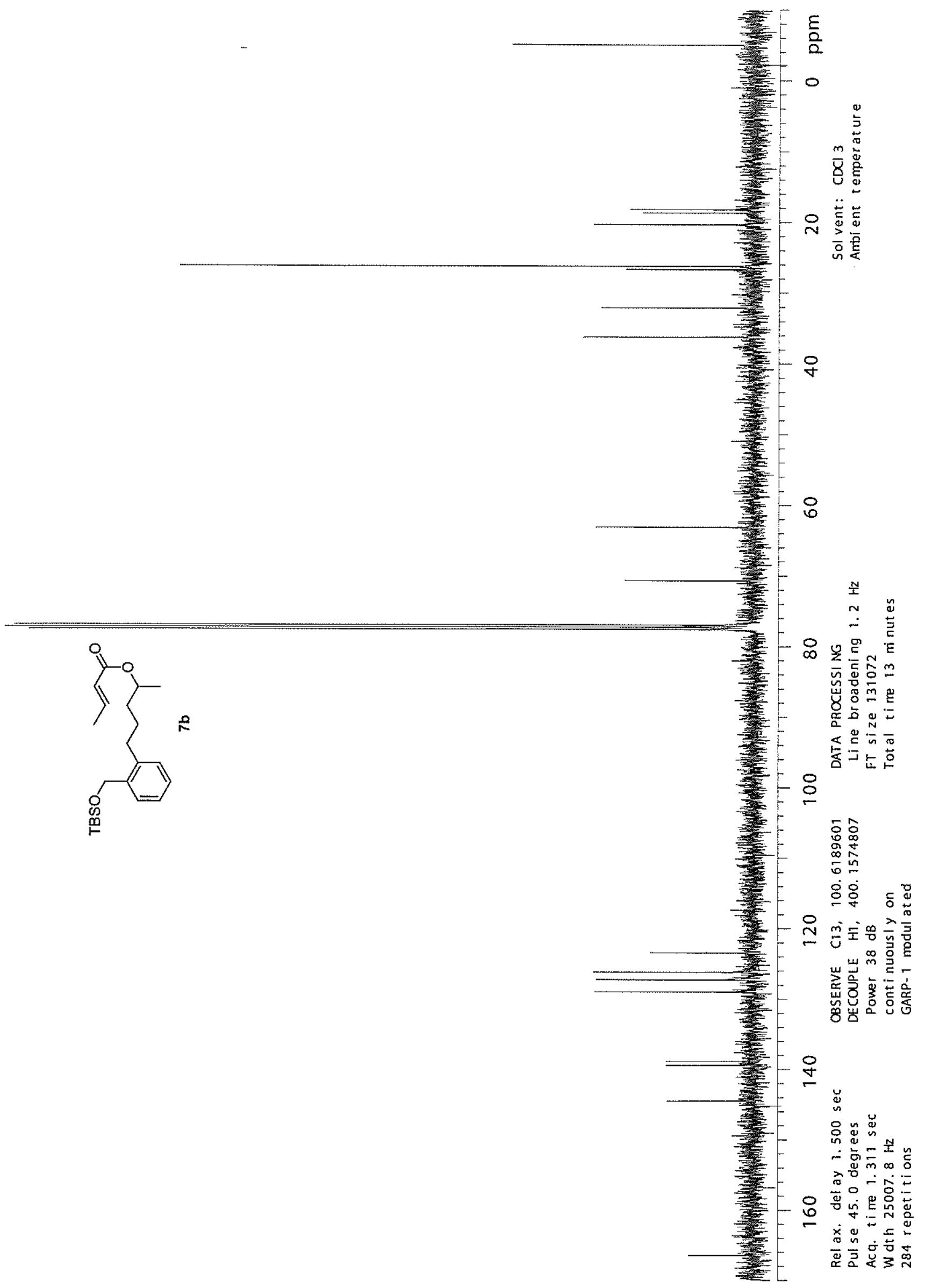




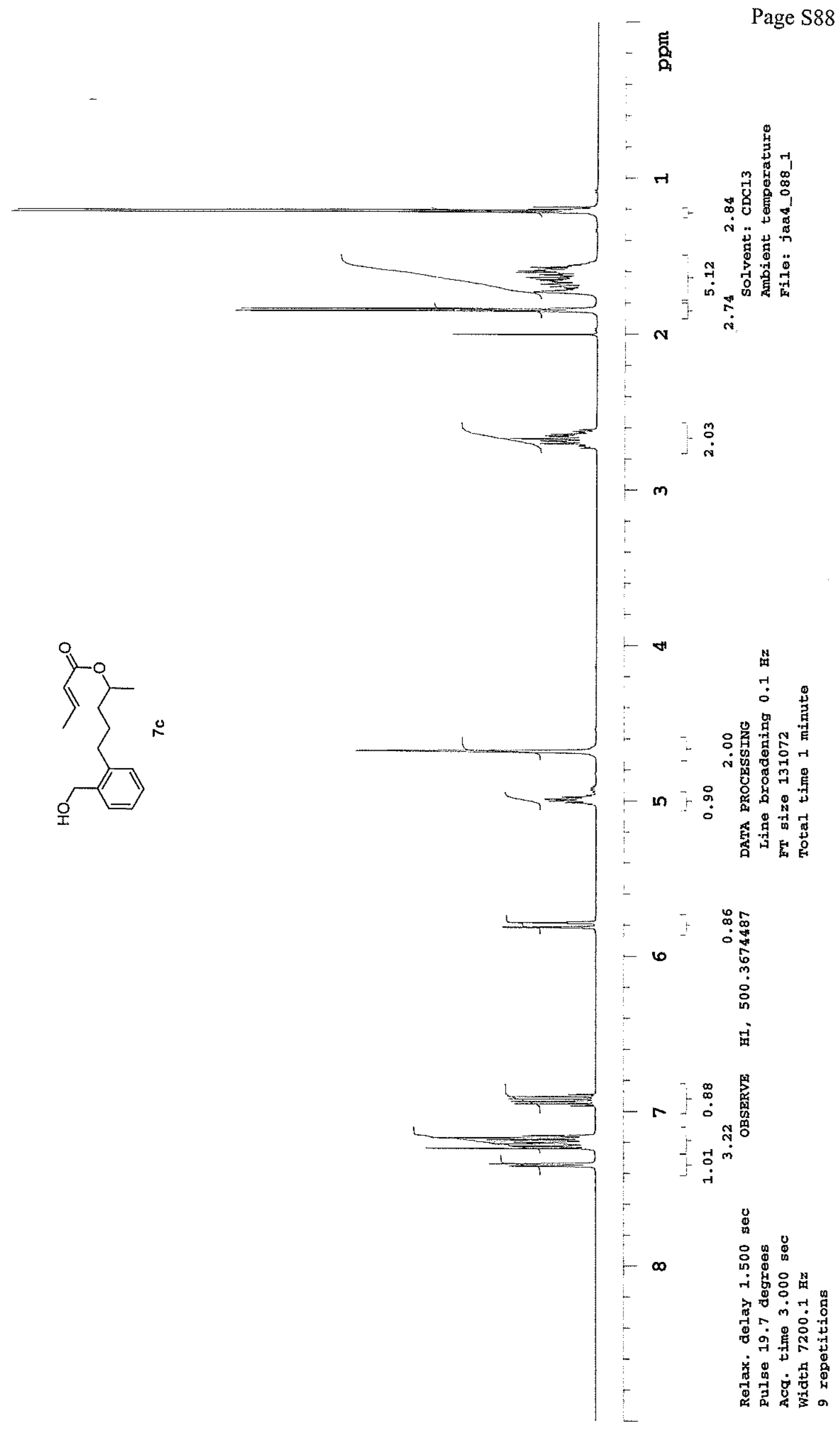




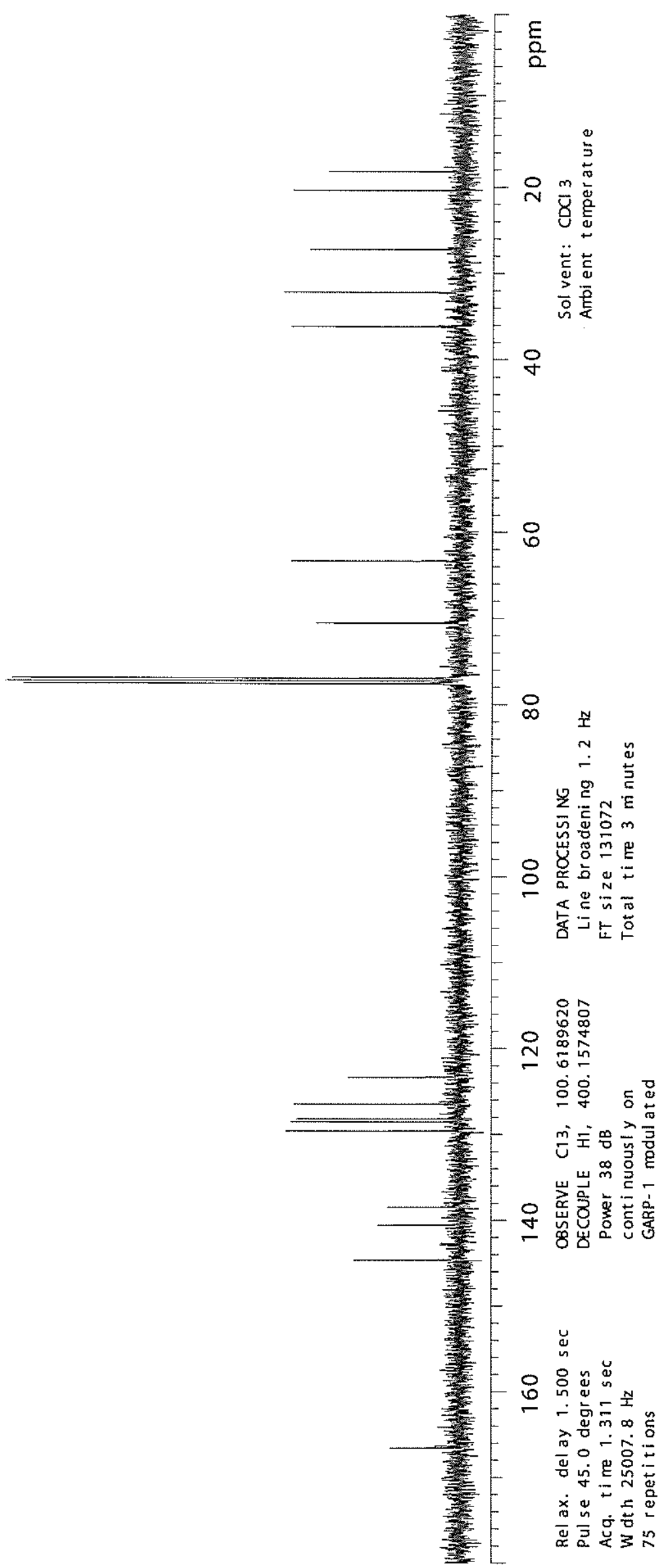



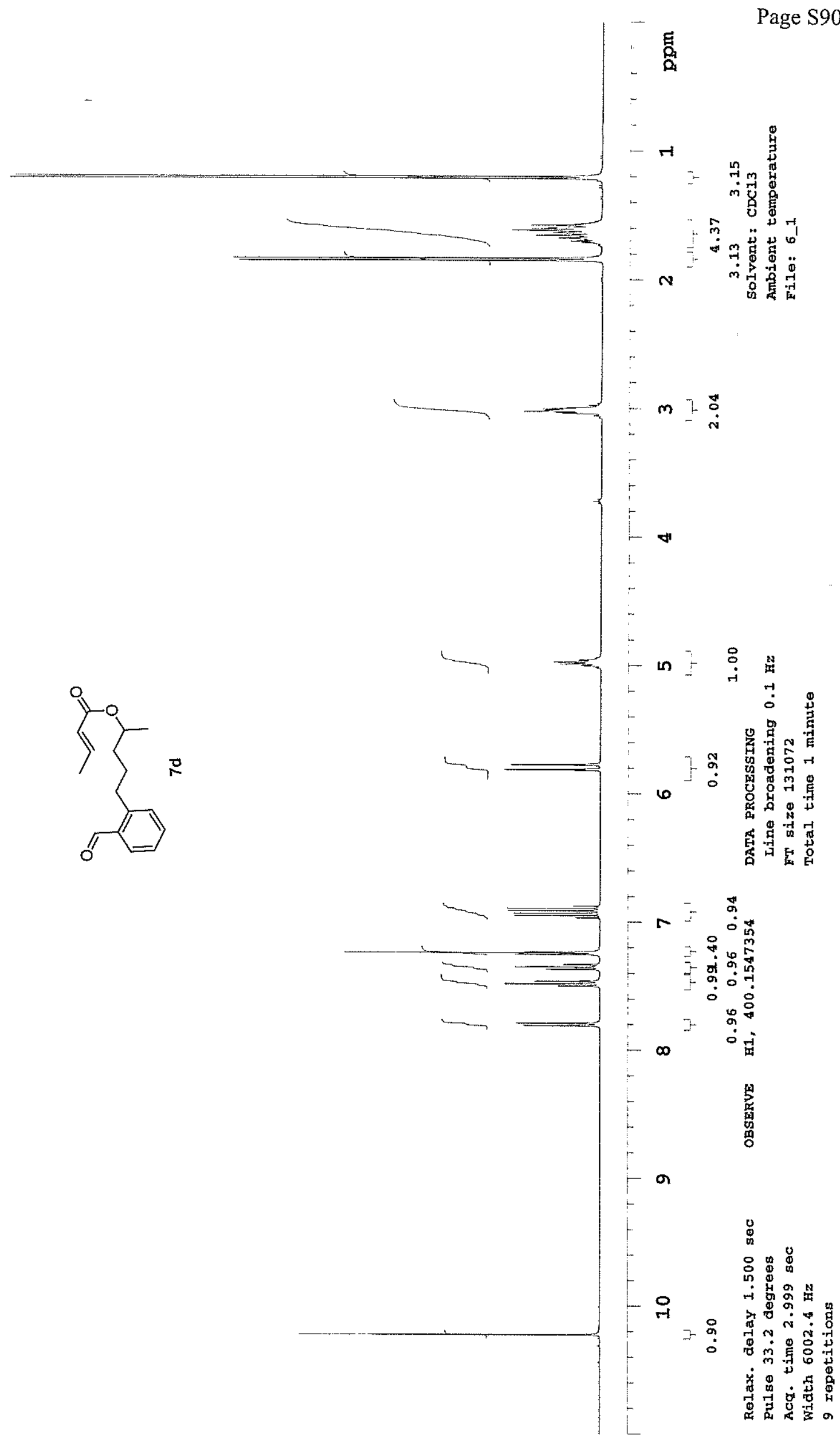


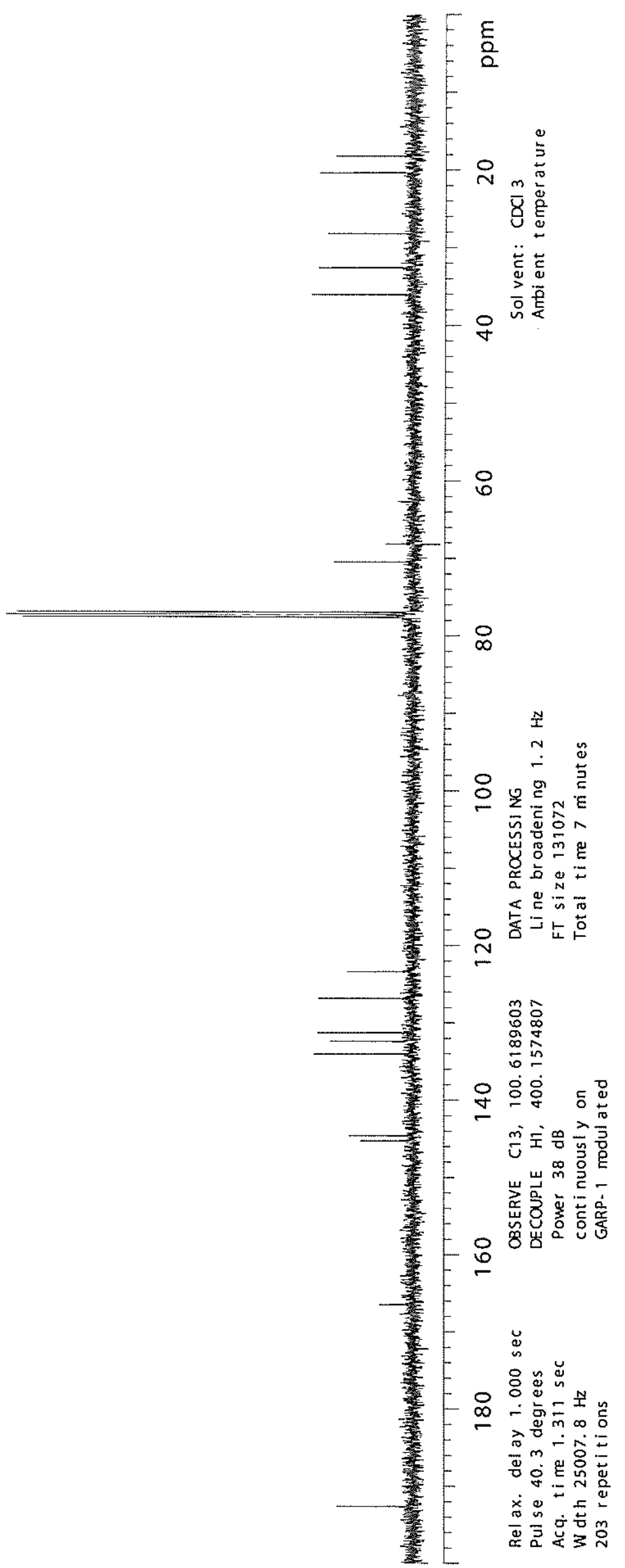




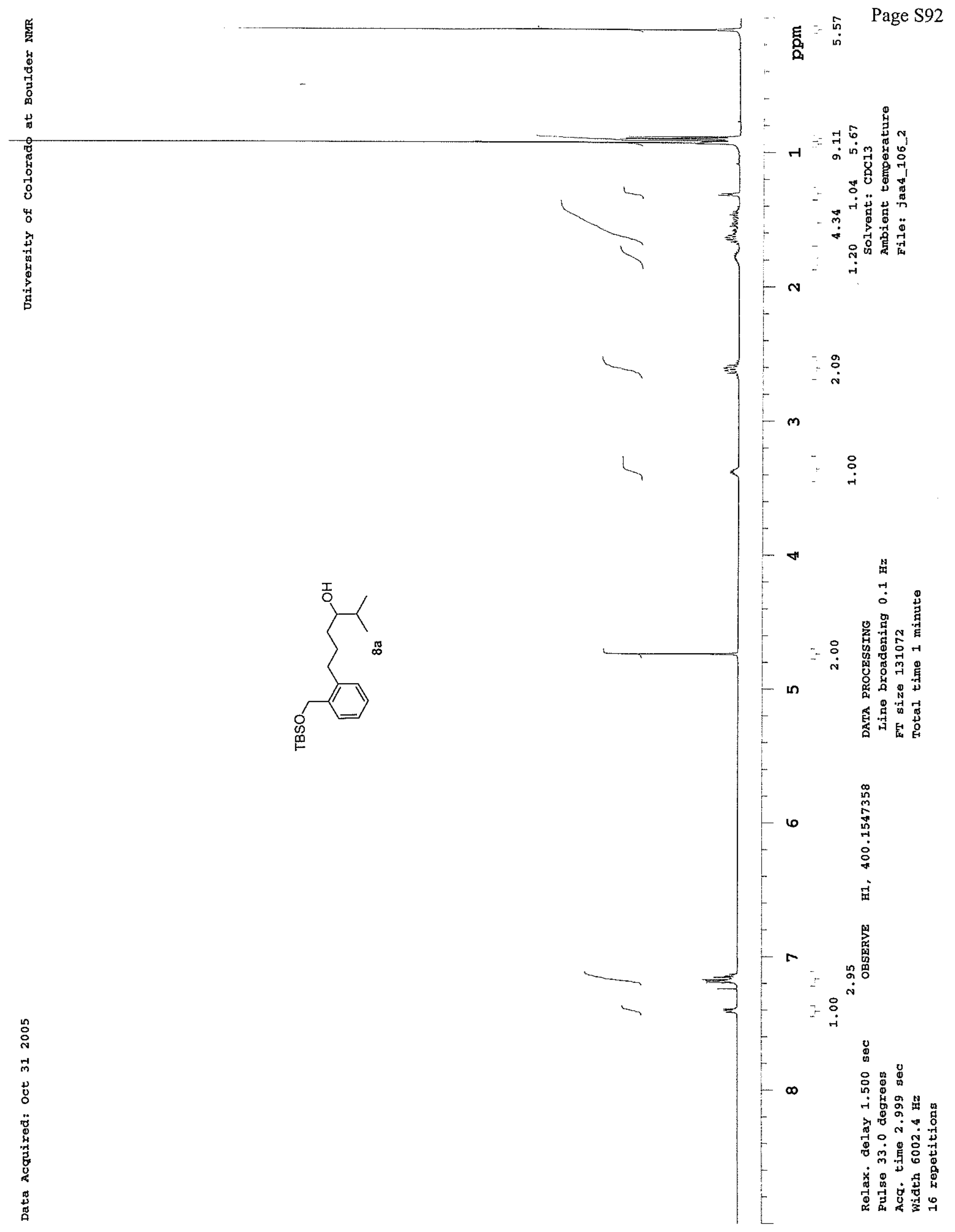




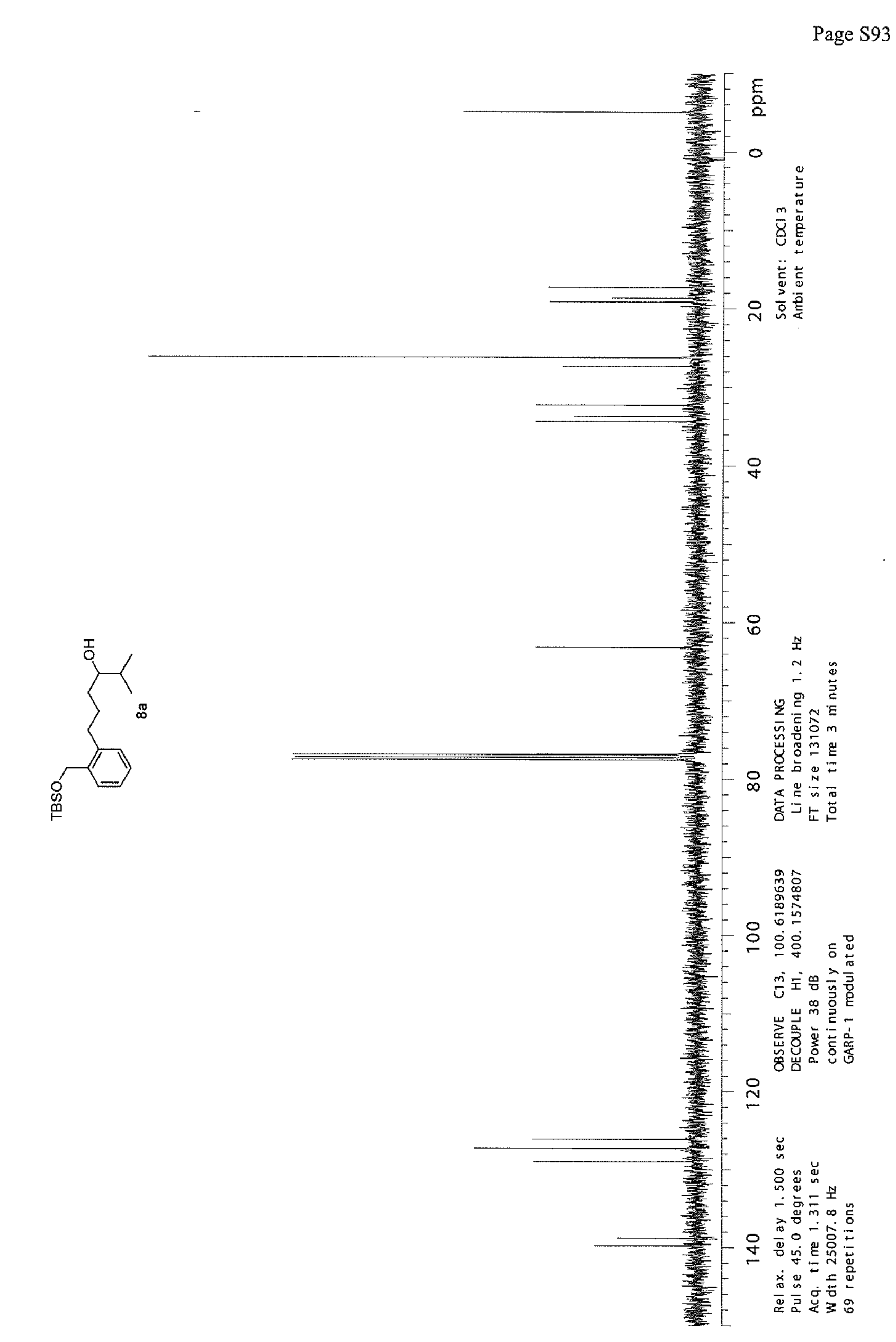




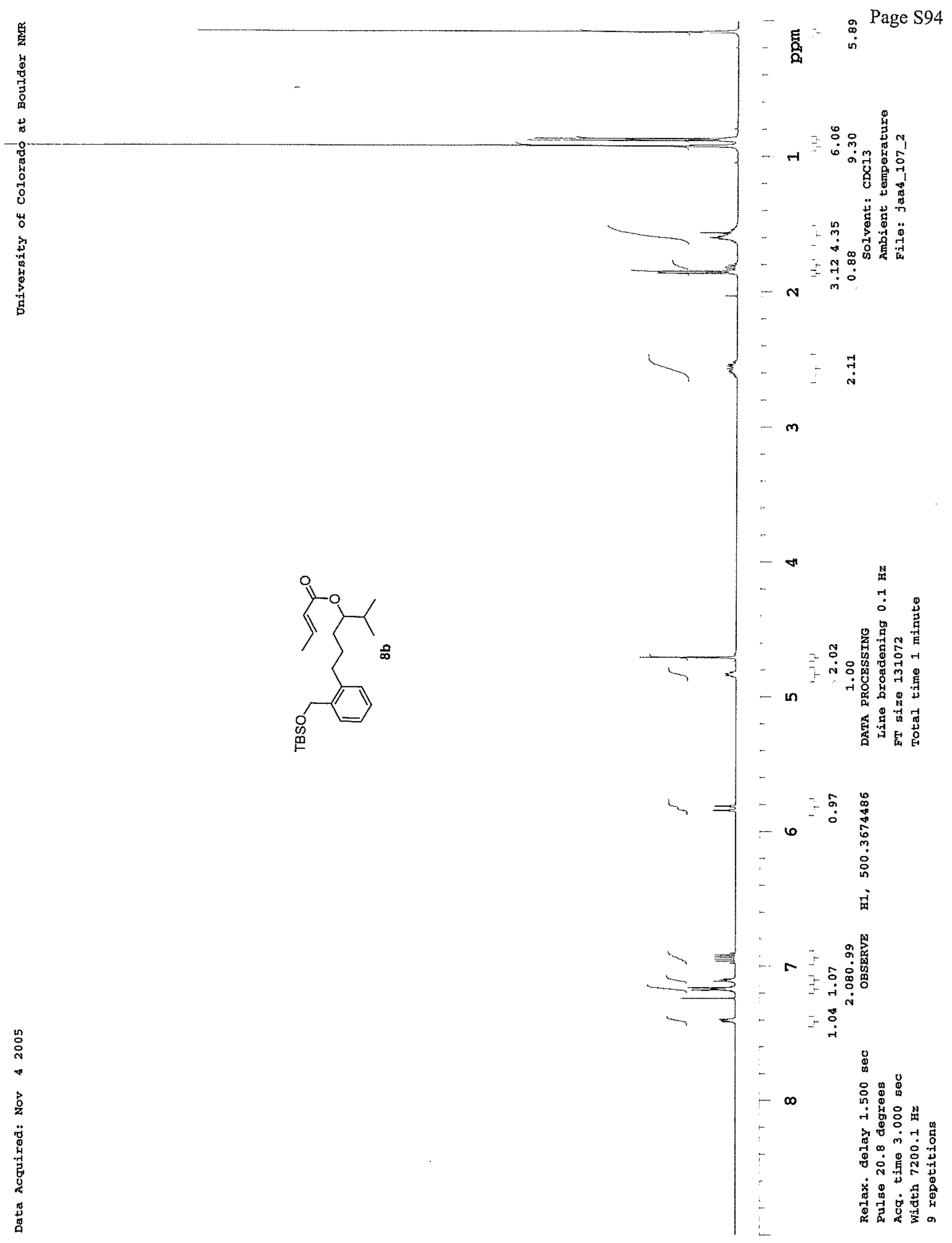


Page S95

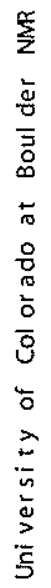

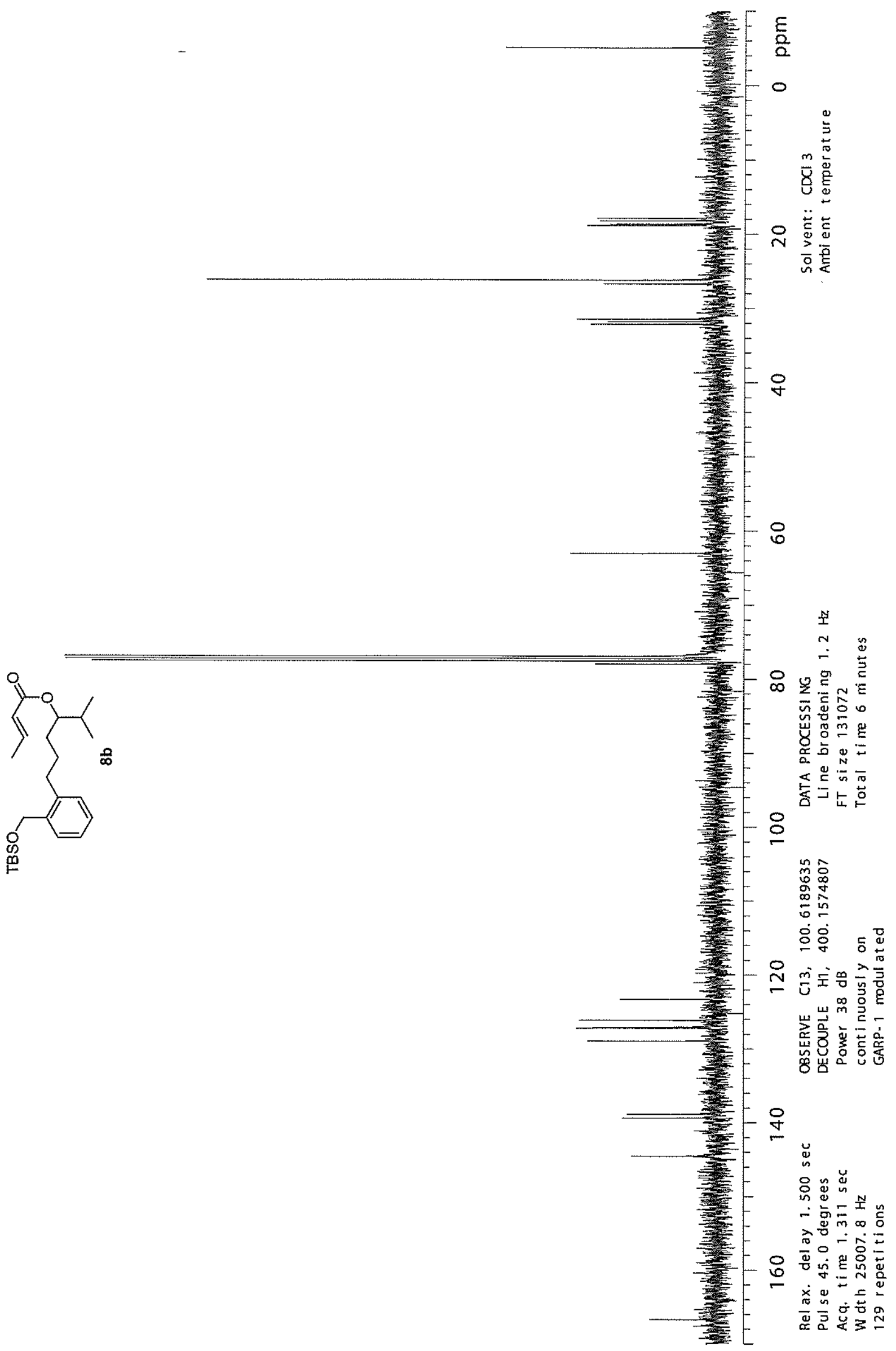




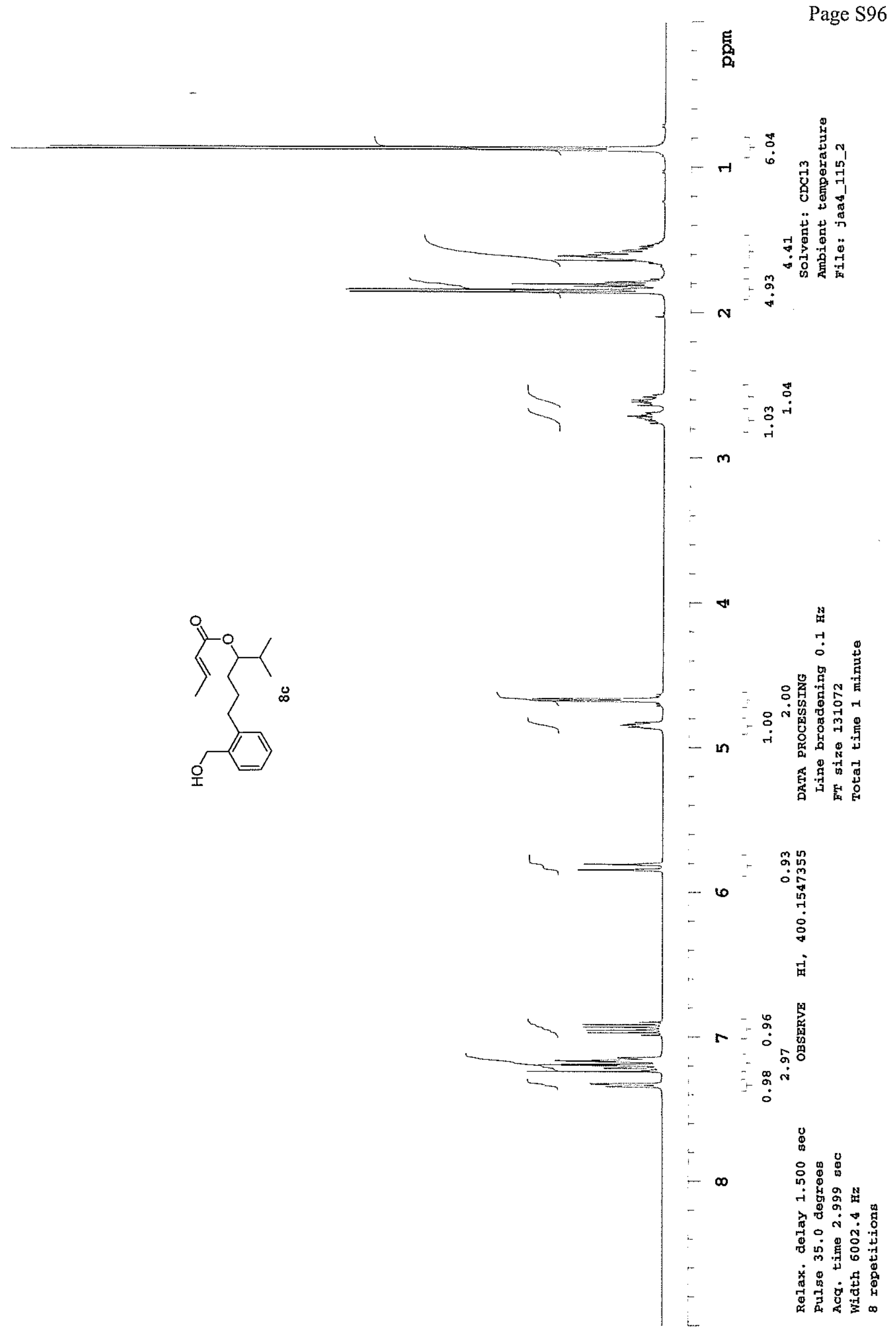




$$
1
$$




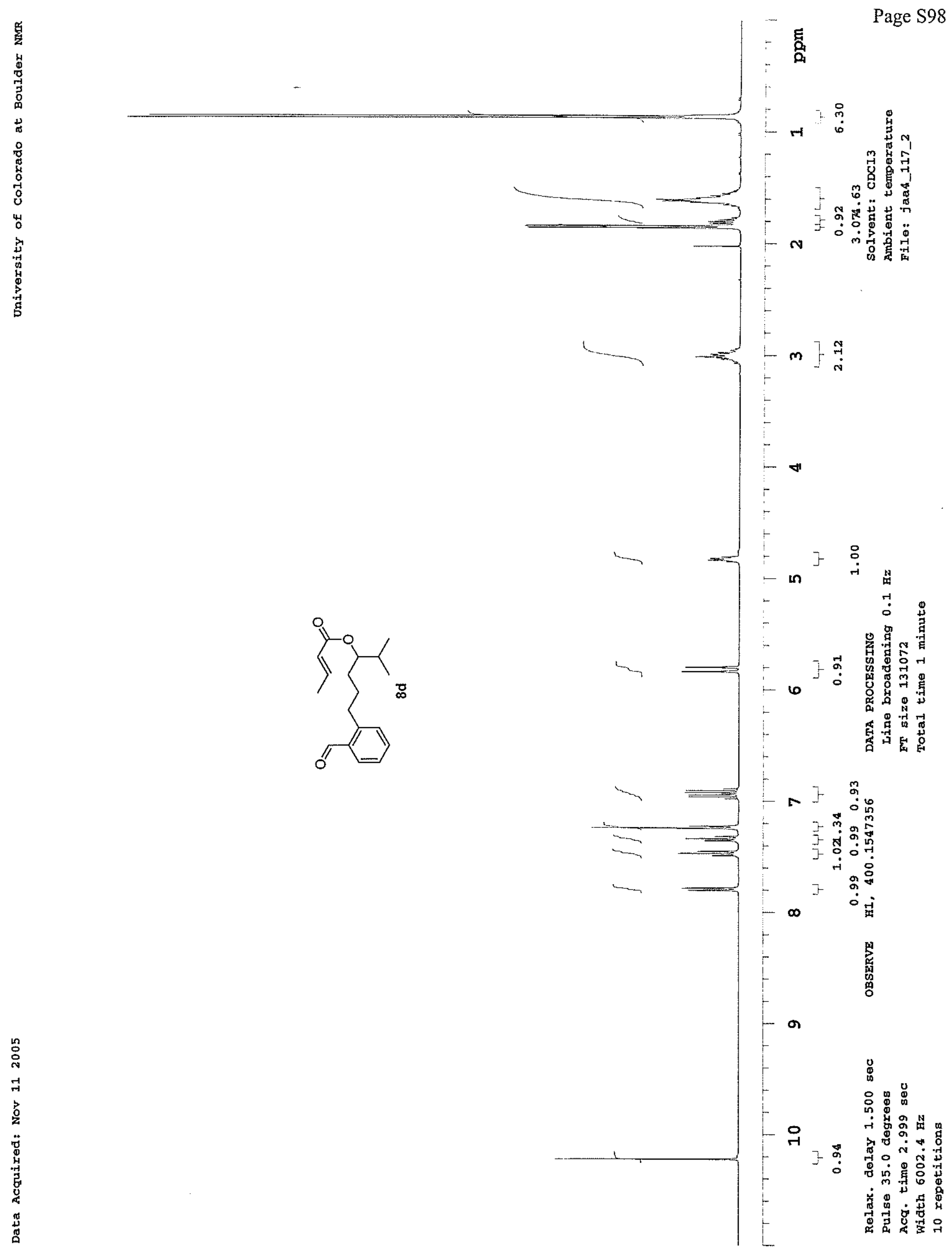




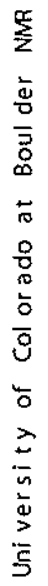
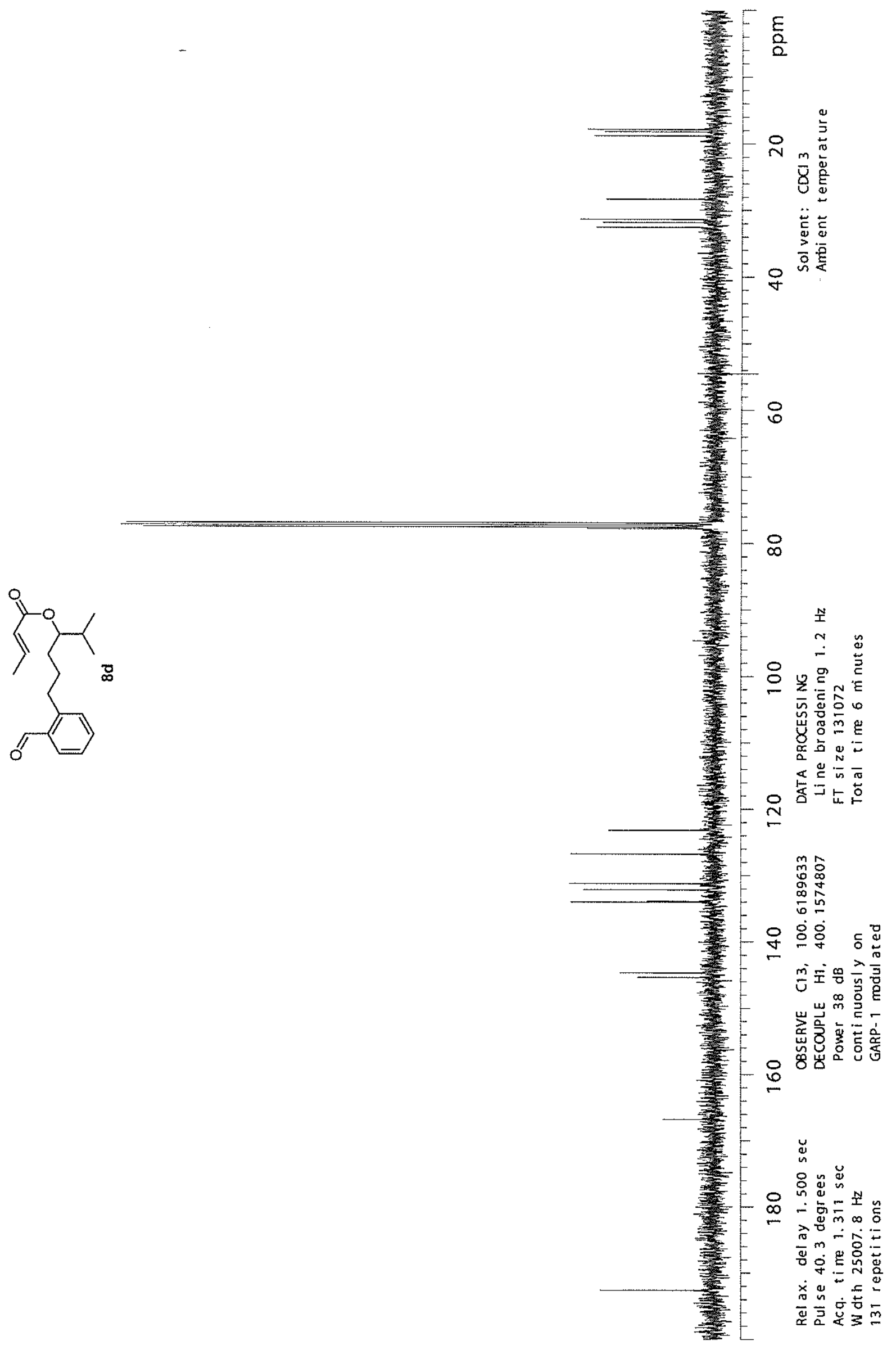


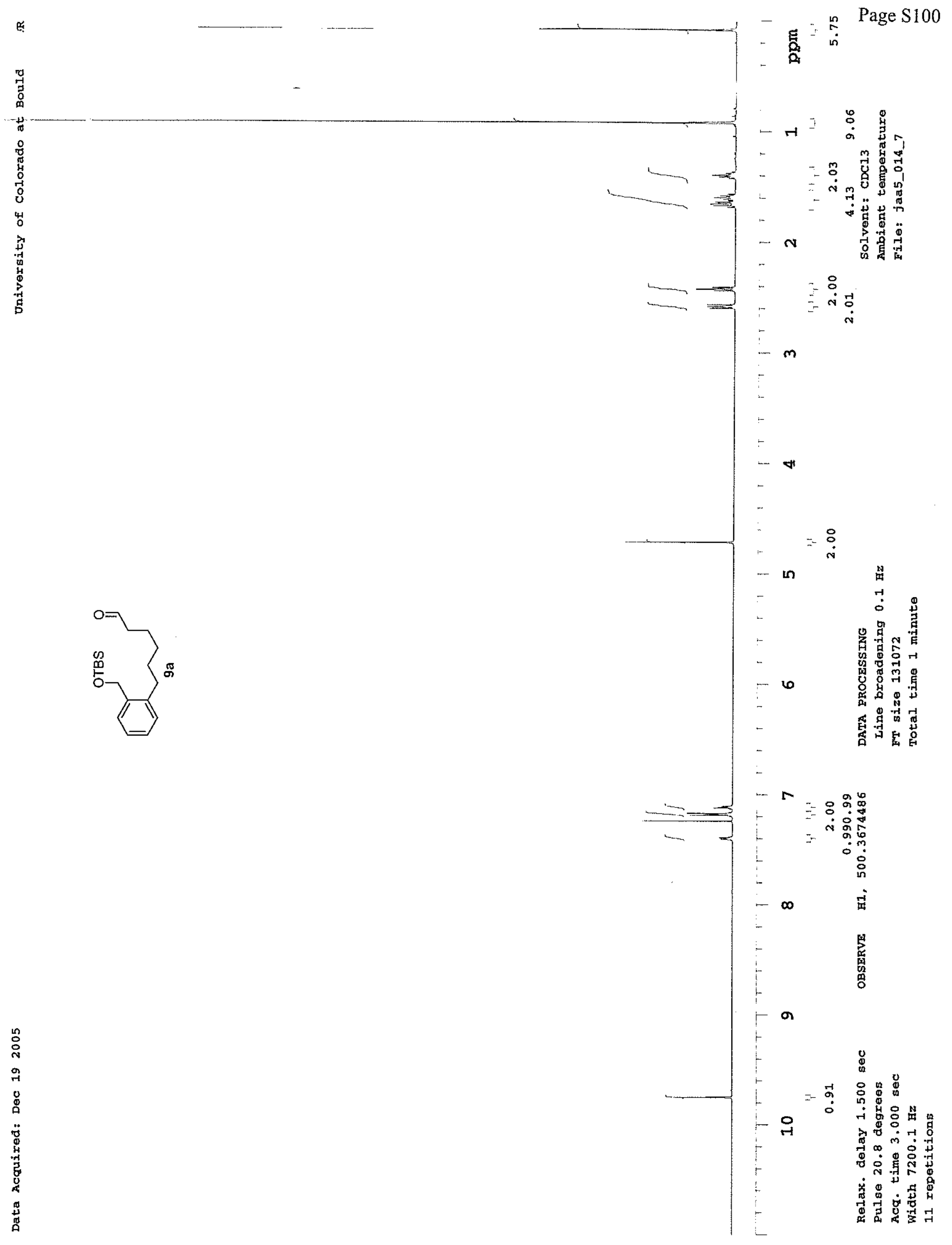


Page S101

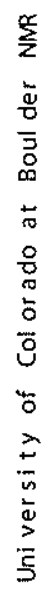

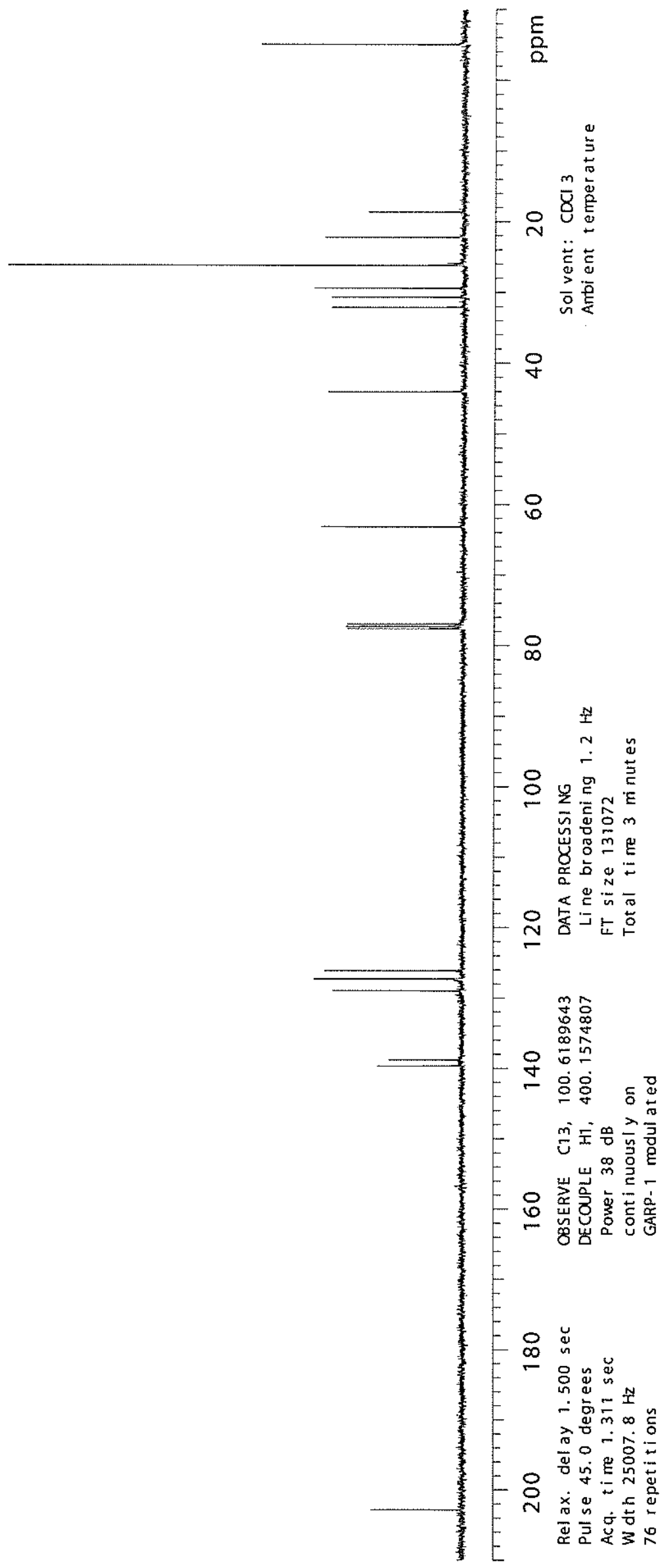




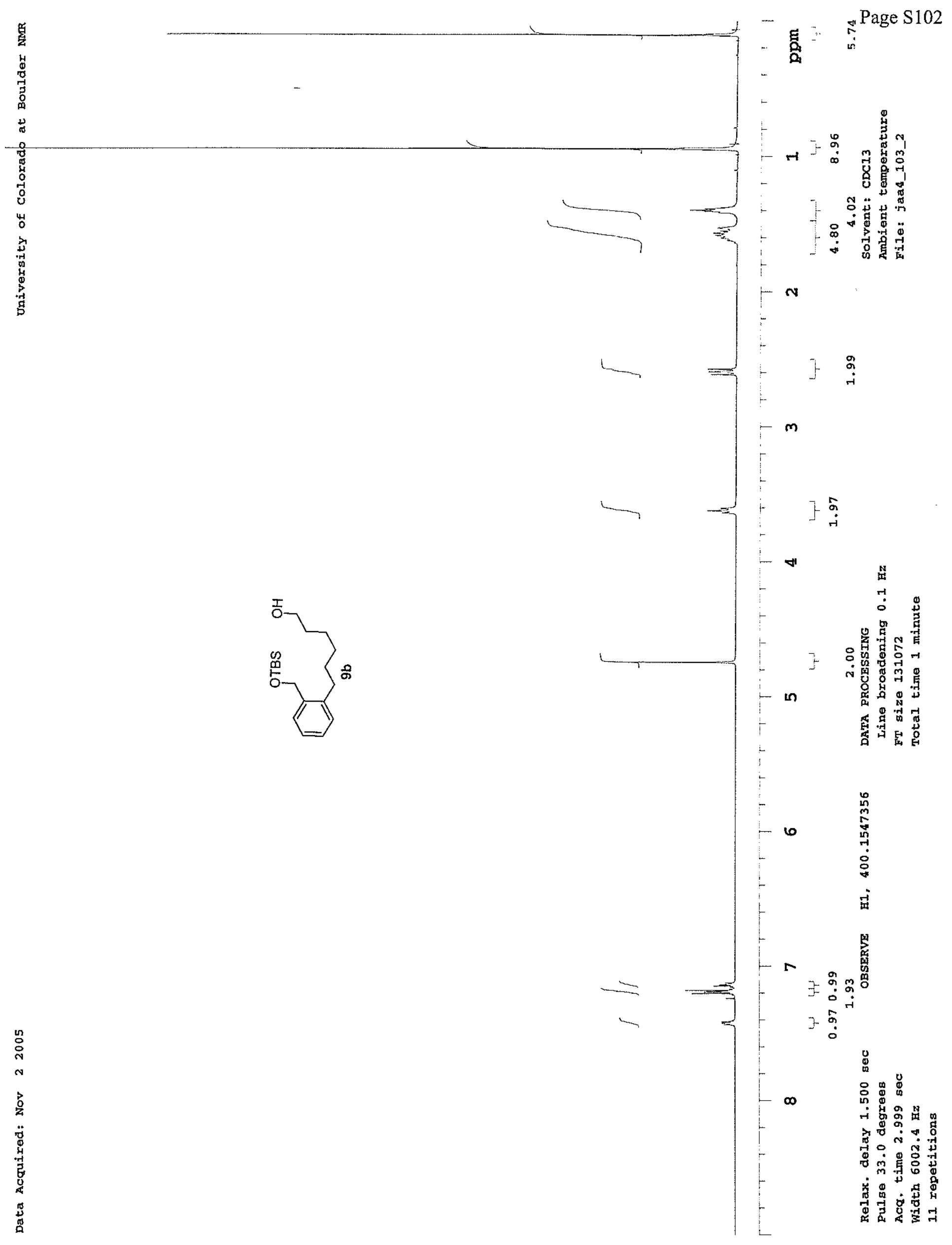


Page S103

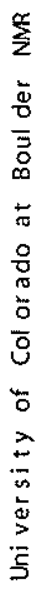

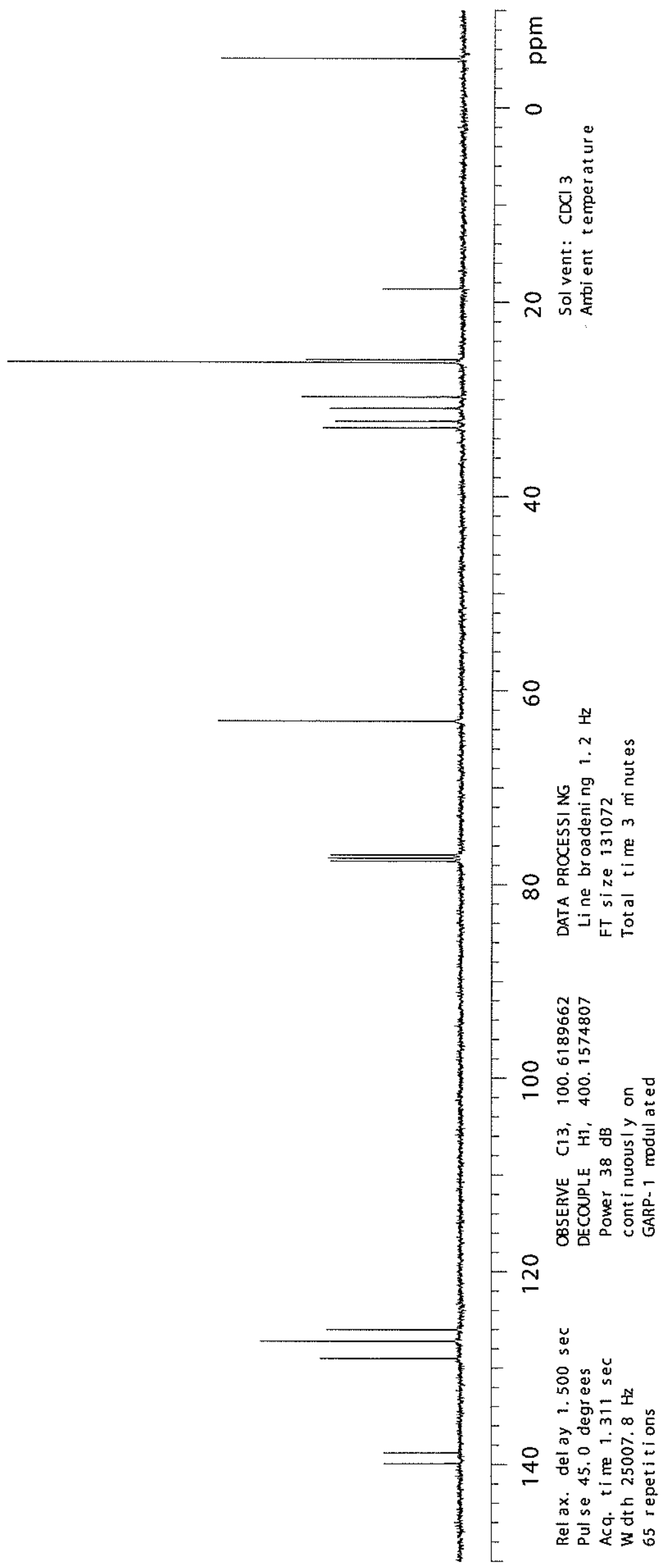




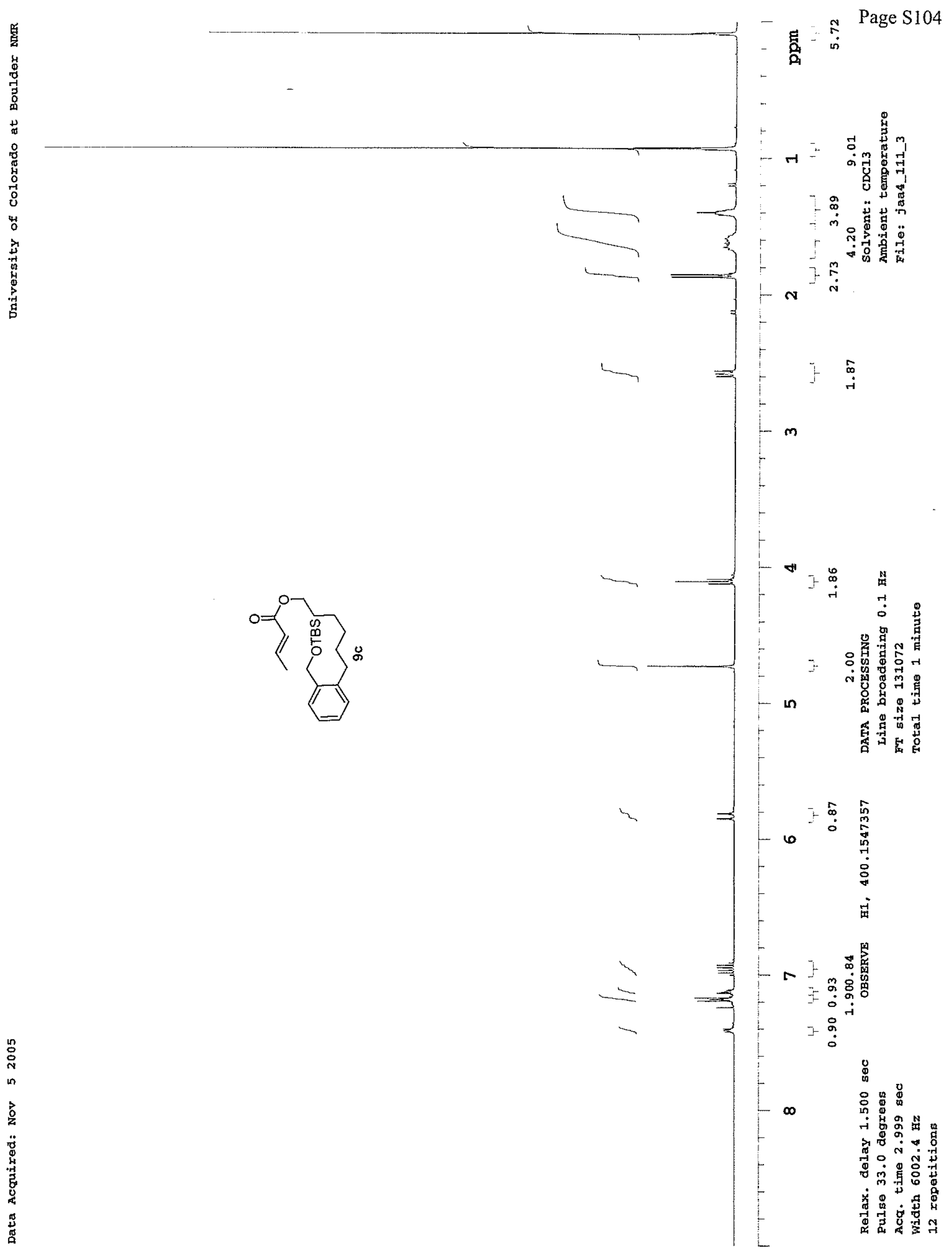


Page $\$ 105$

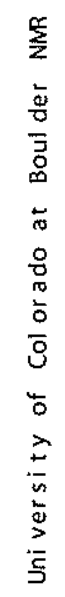

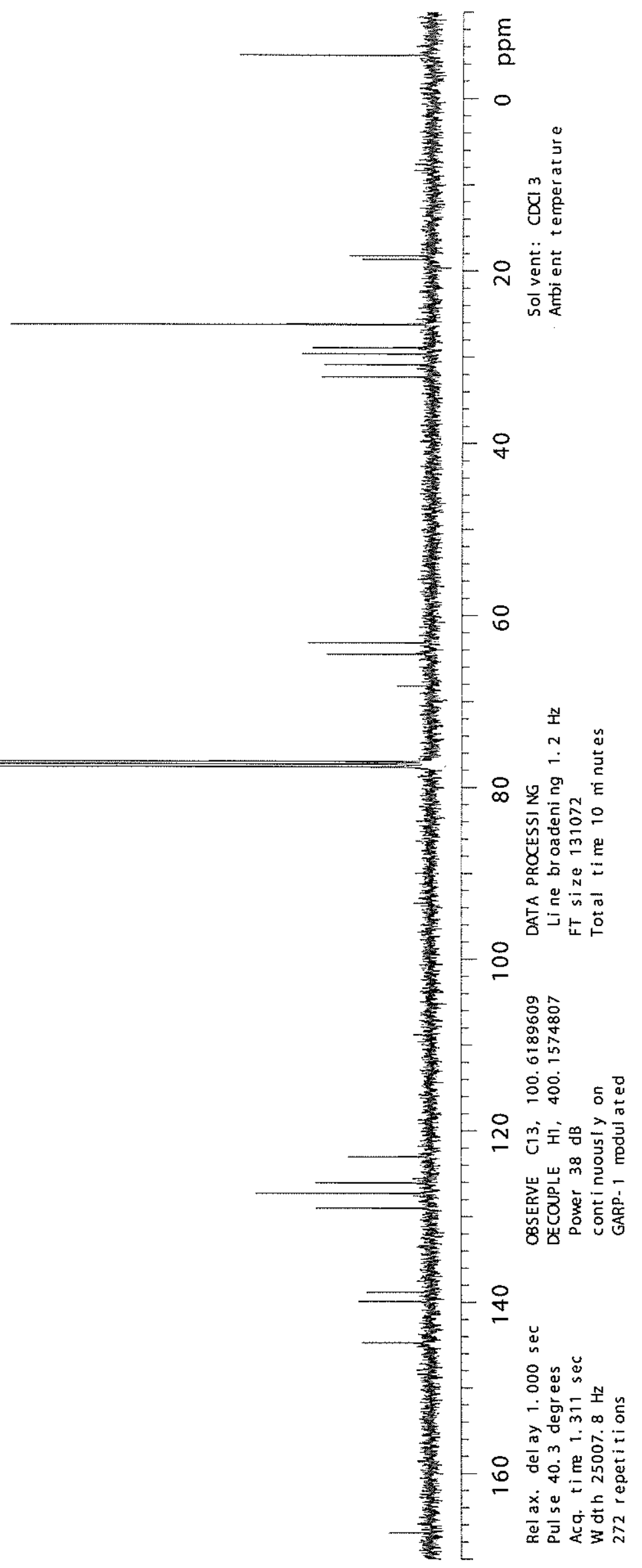




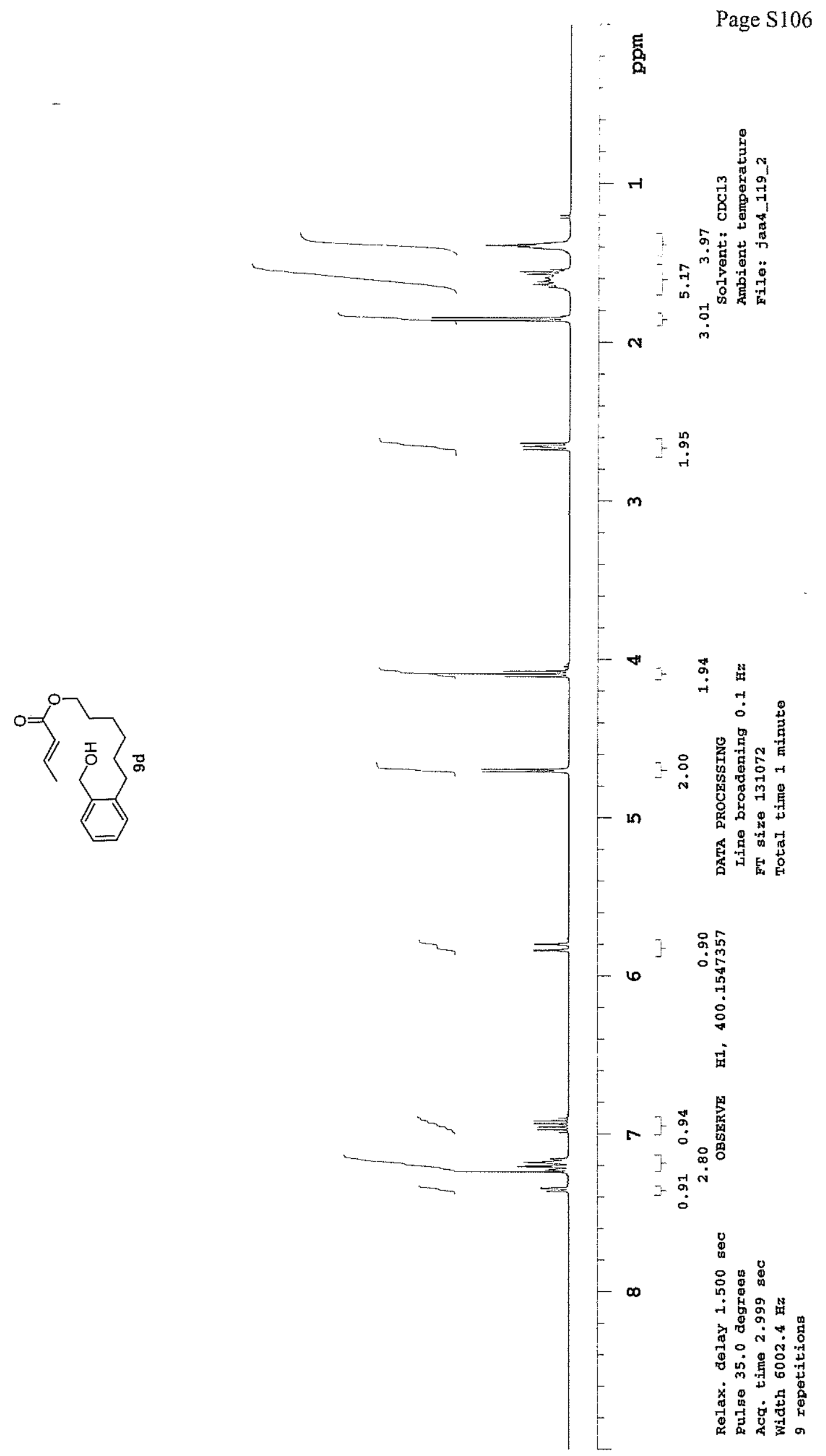


Page S107

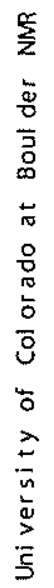

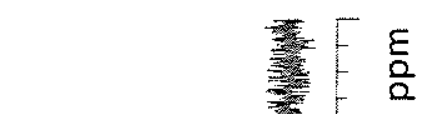

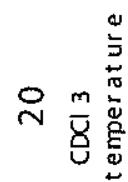

茝

용

$\stackrel{0}{8}$

8

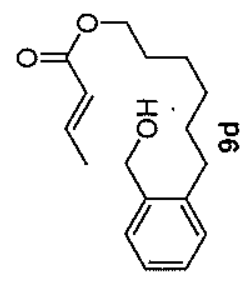

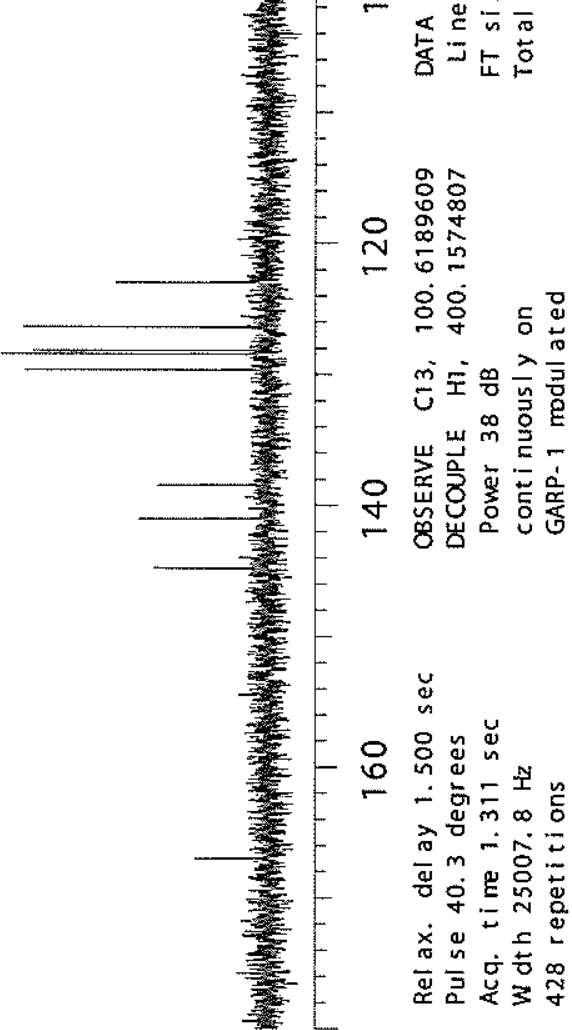




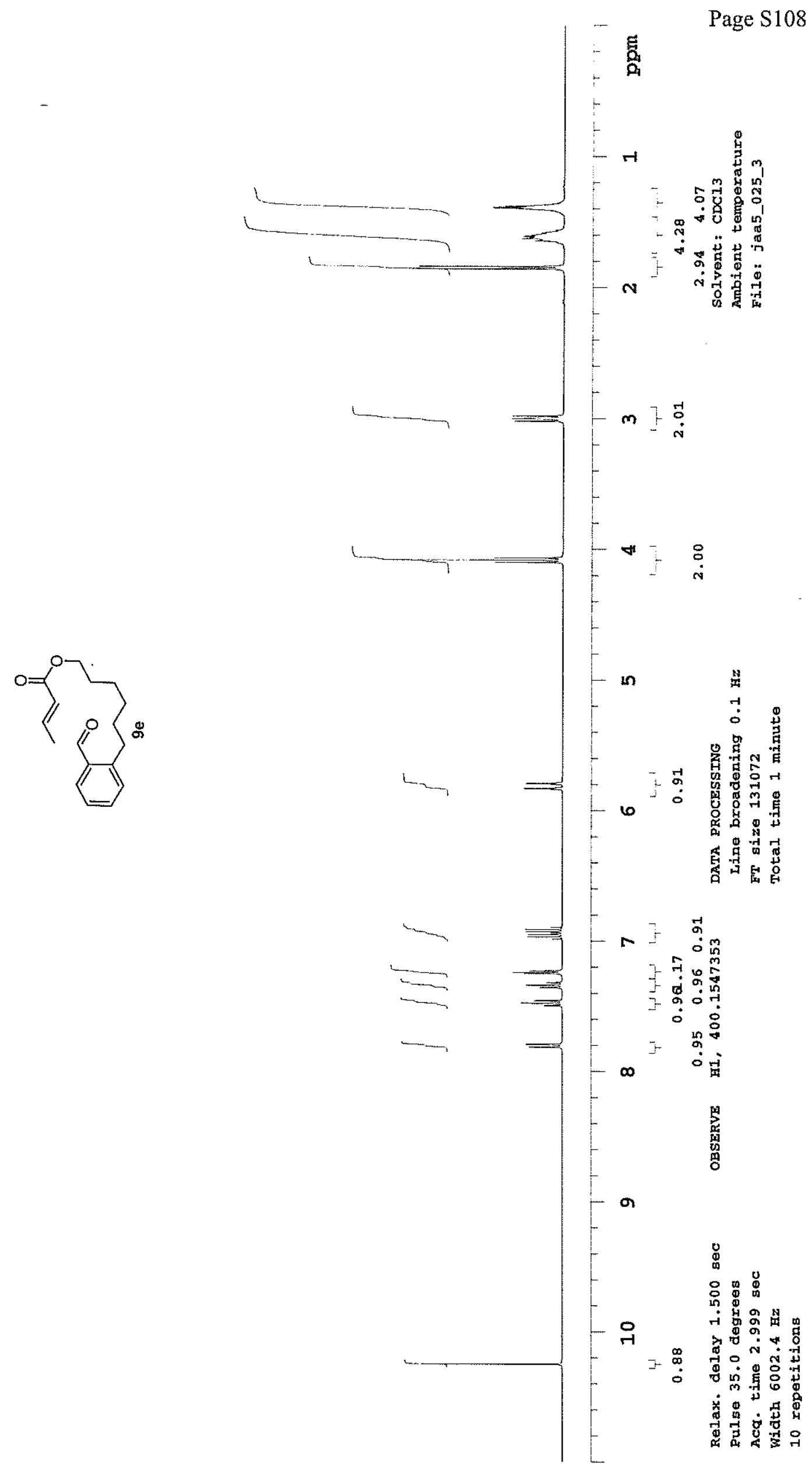


Page S109

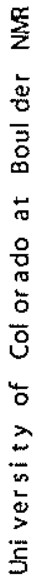

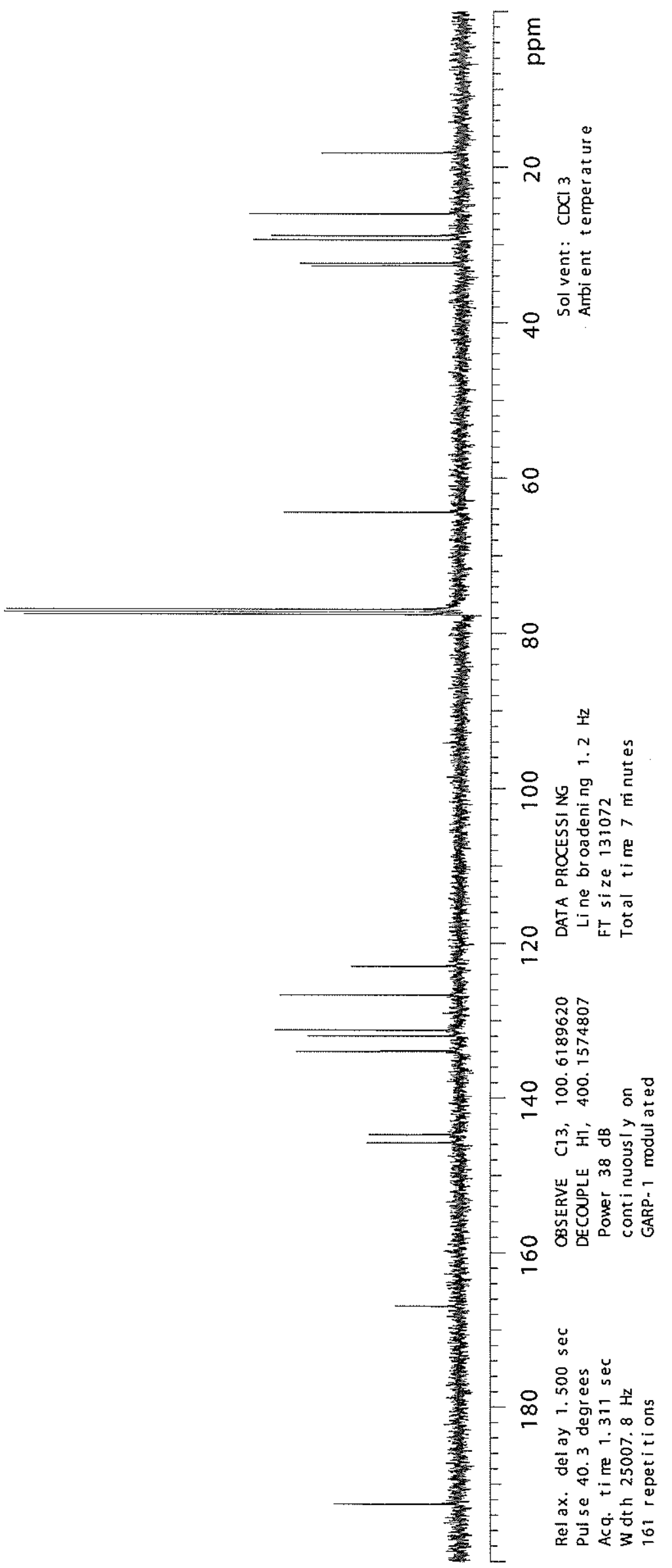




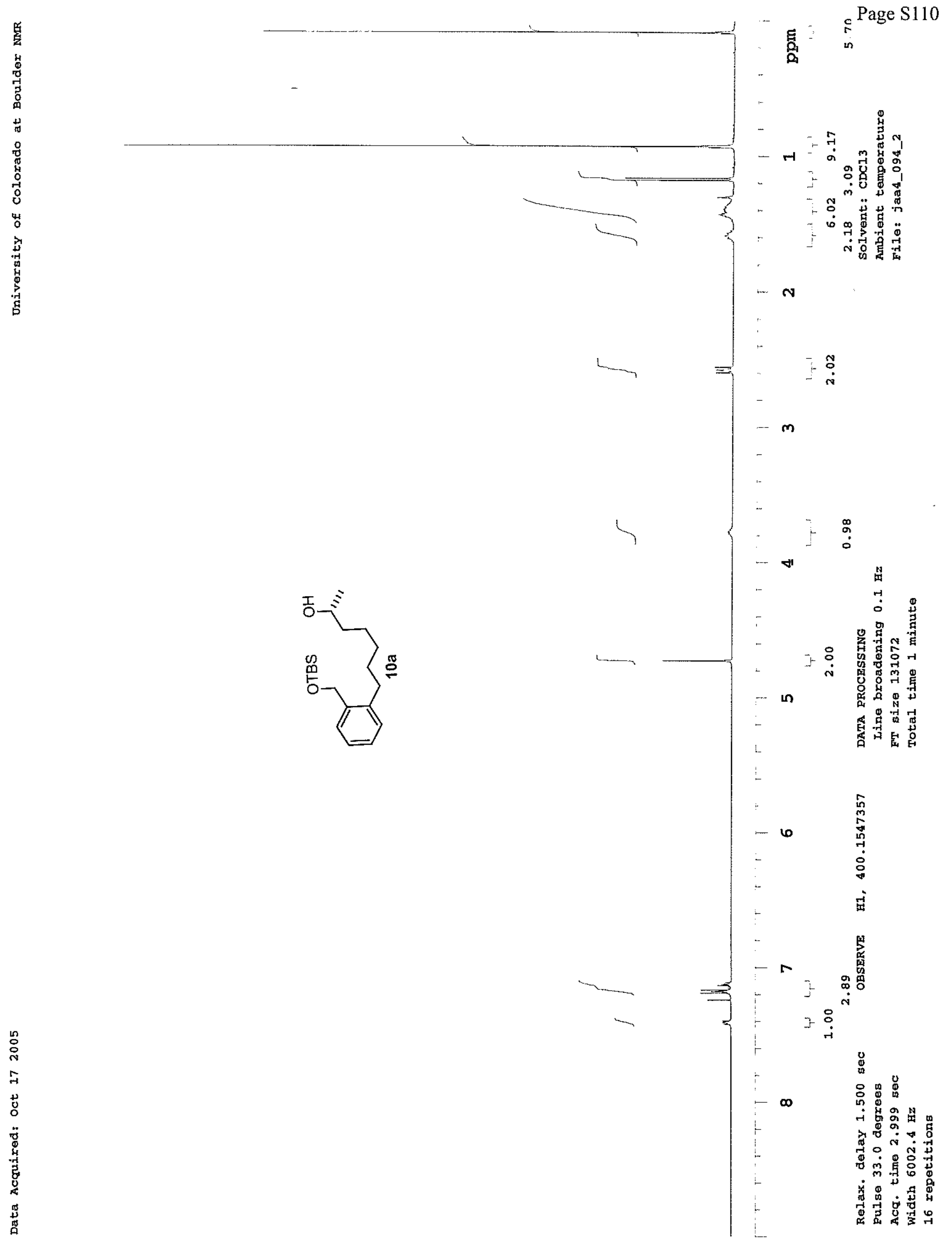


Page S111

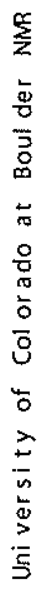

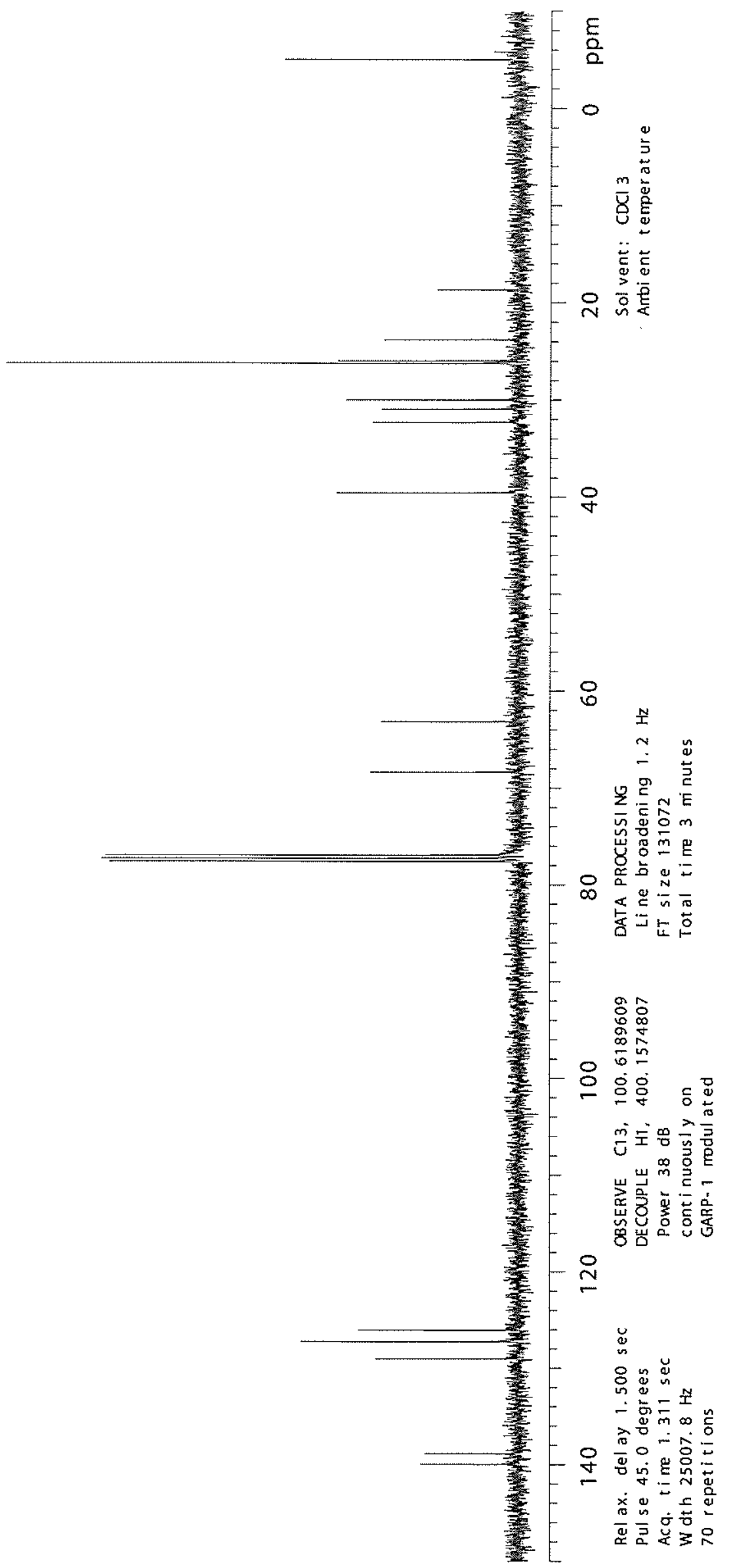




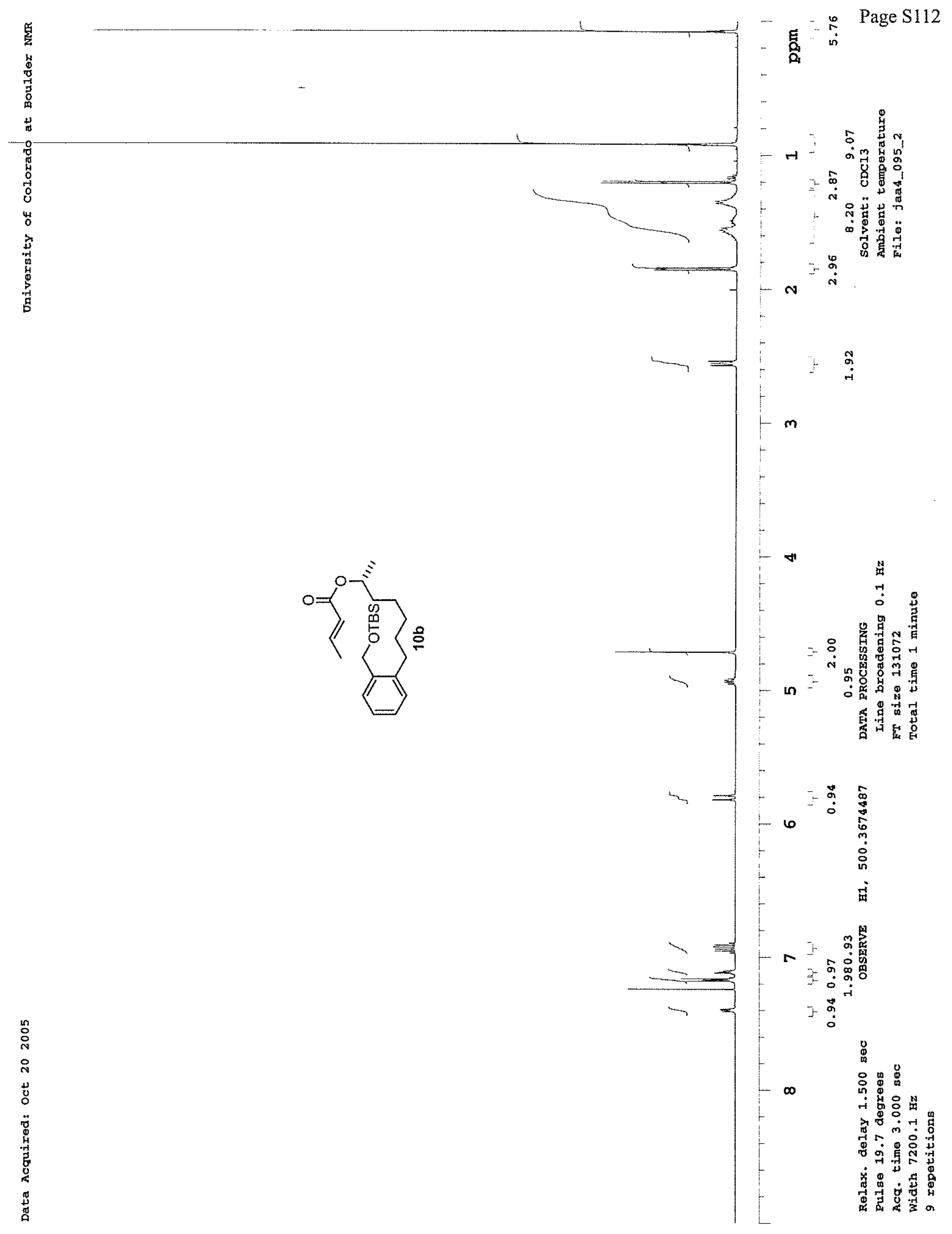


Page S113

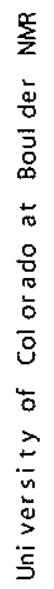

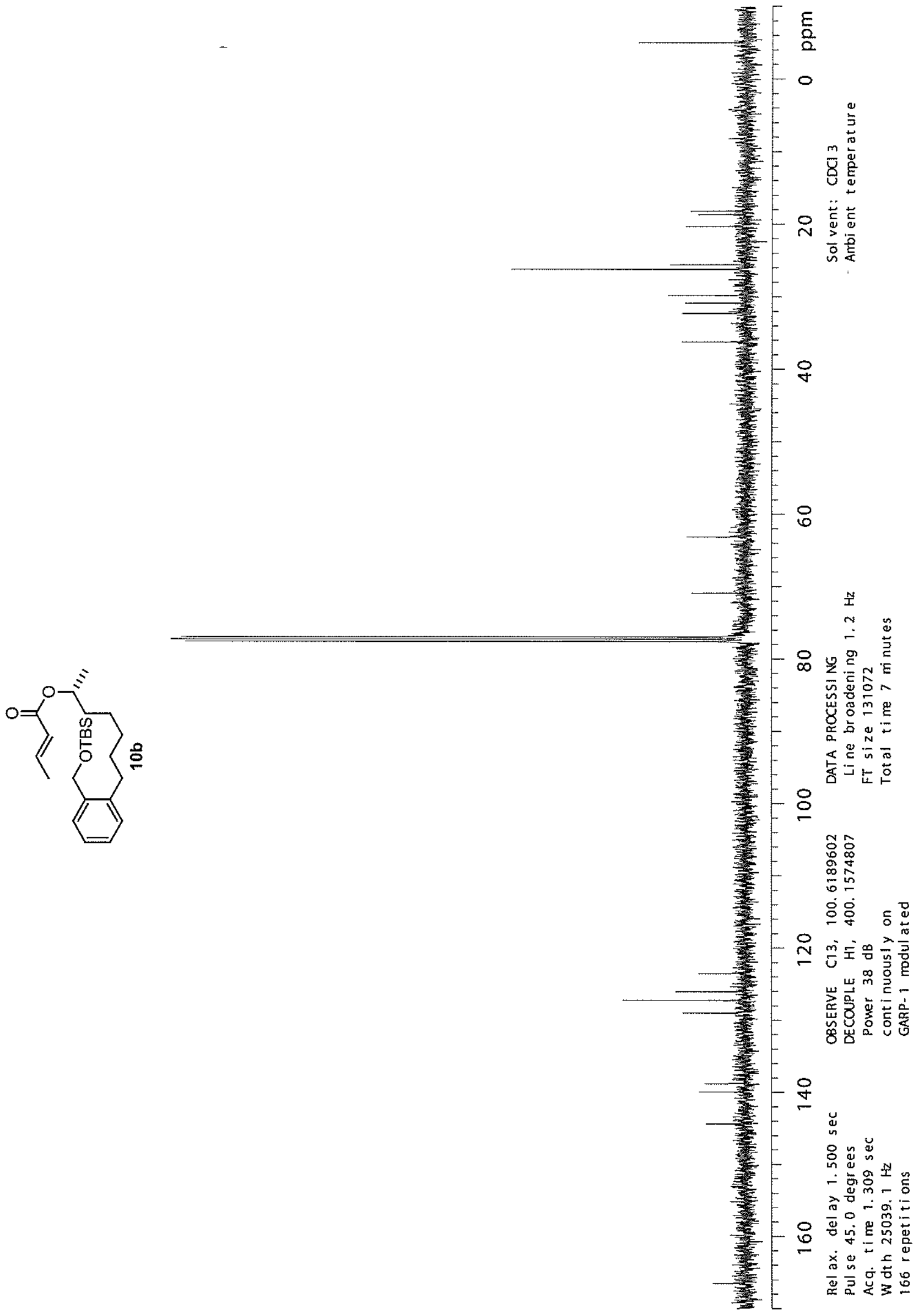



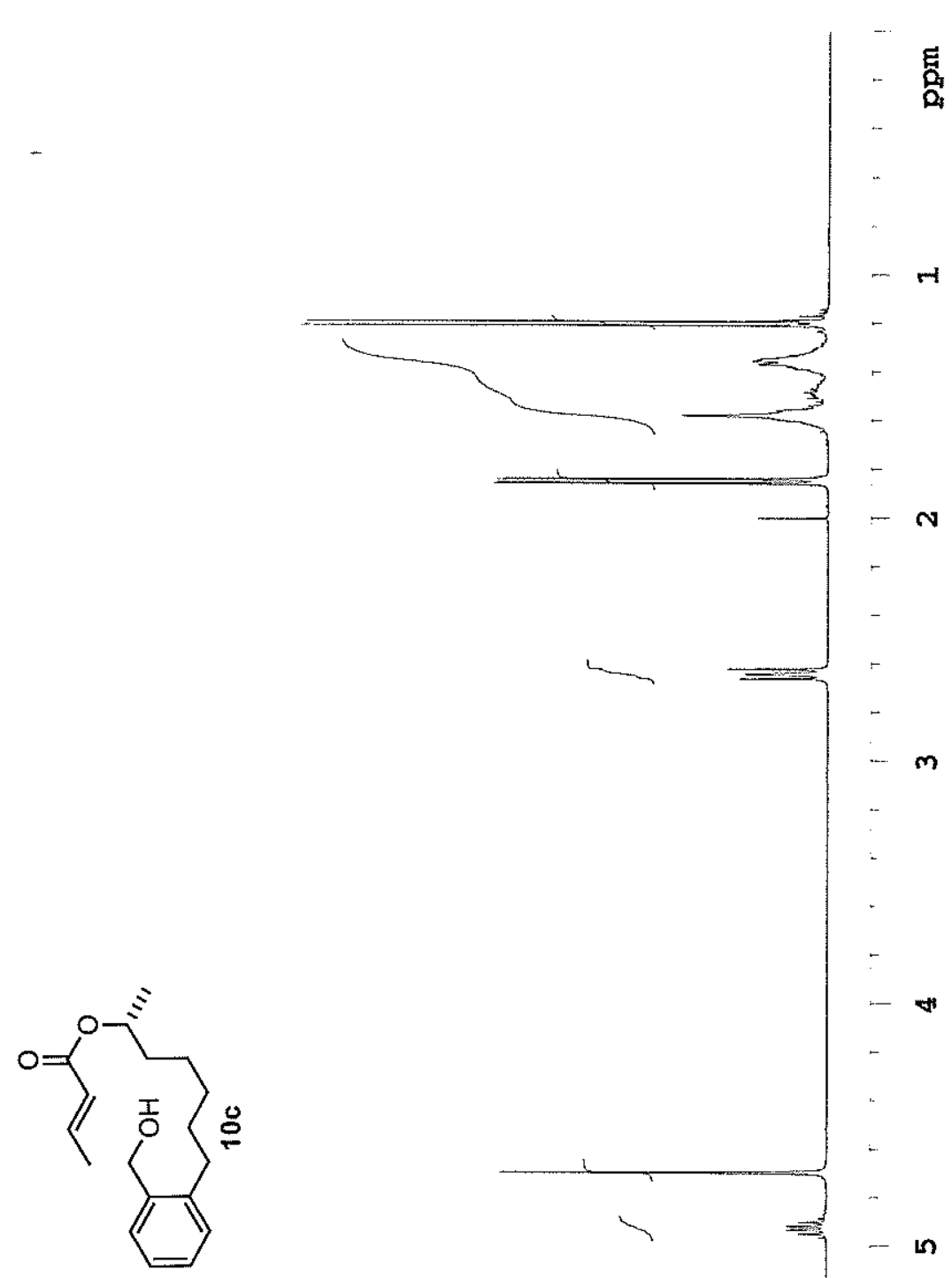

吕
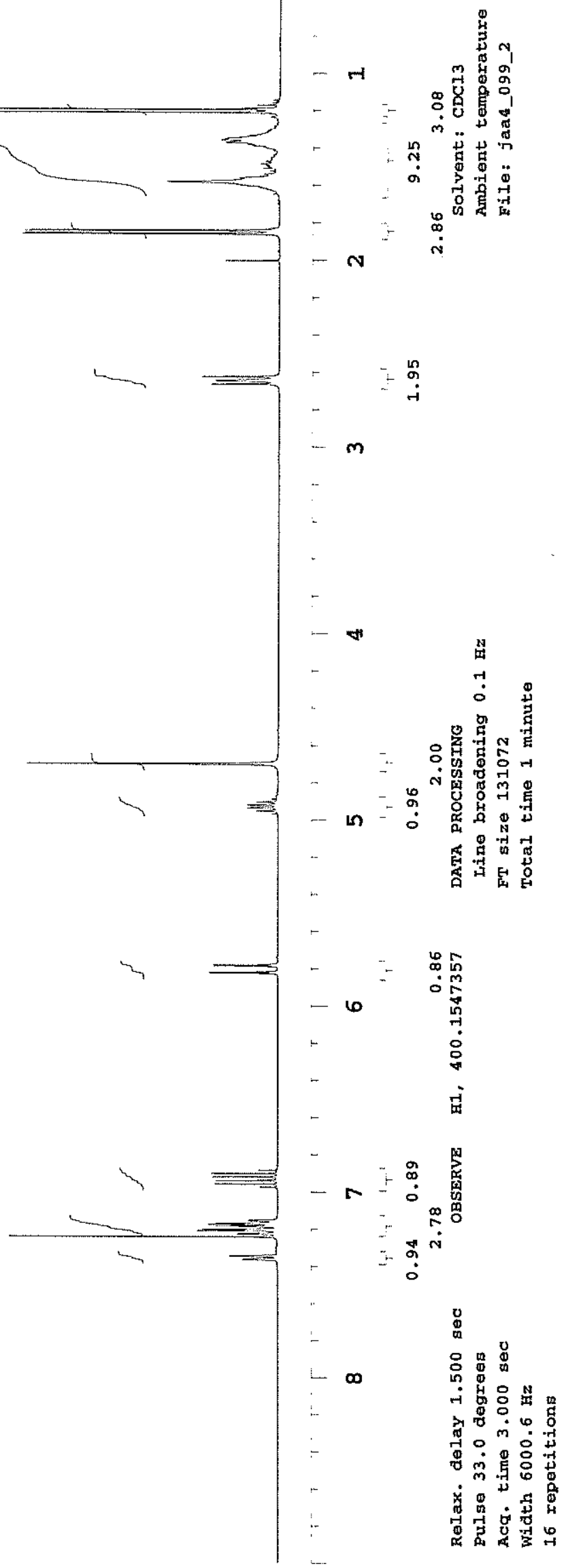
Page S115

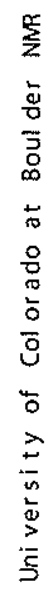
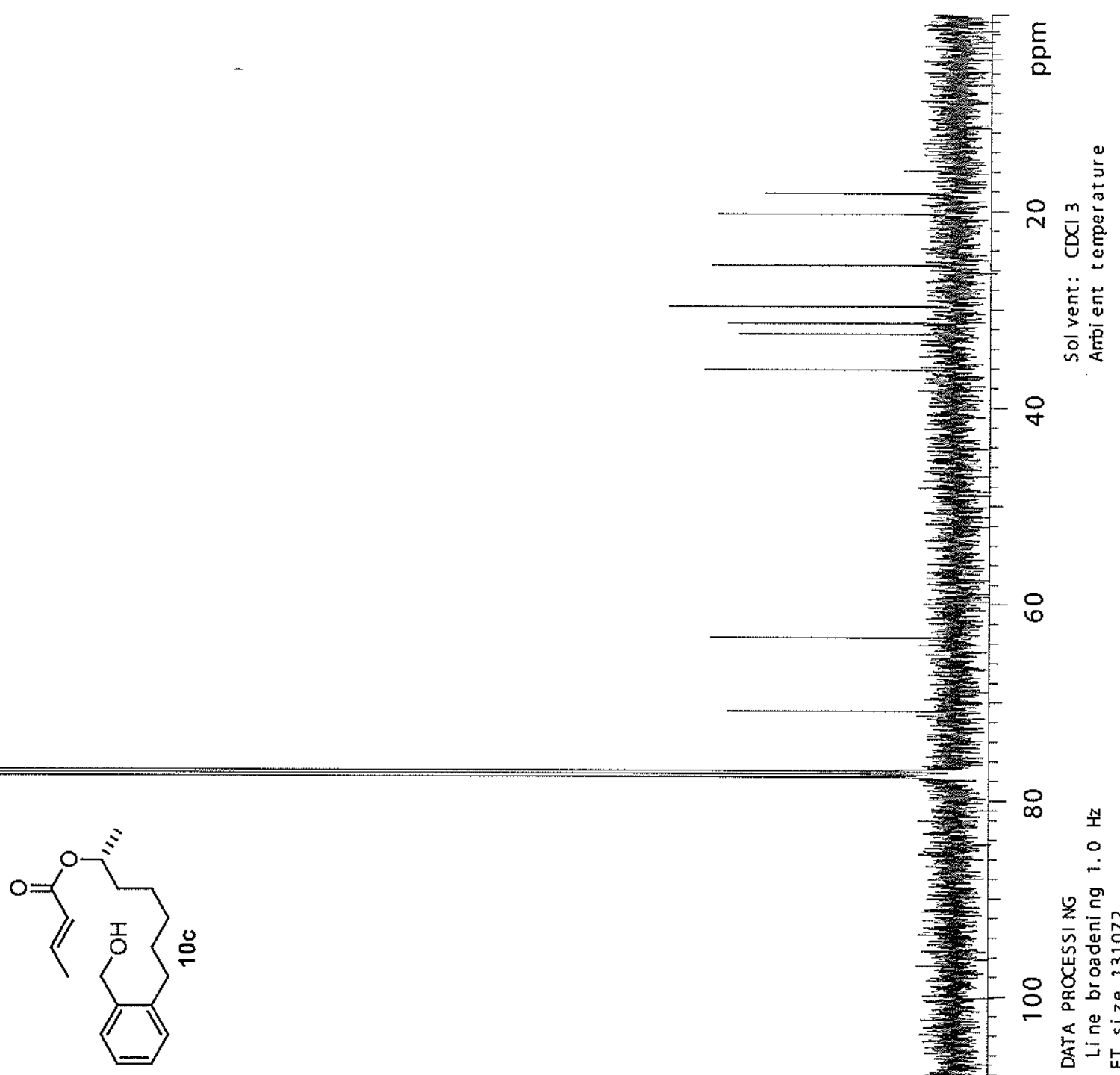

$\infty$

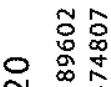

는

实安 ᄃ

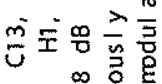

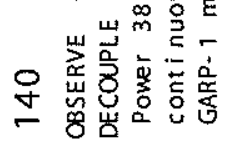

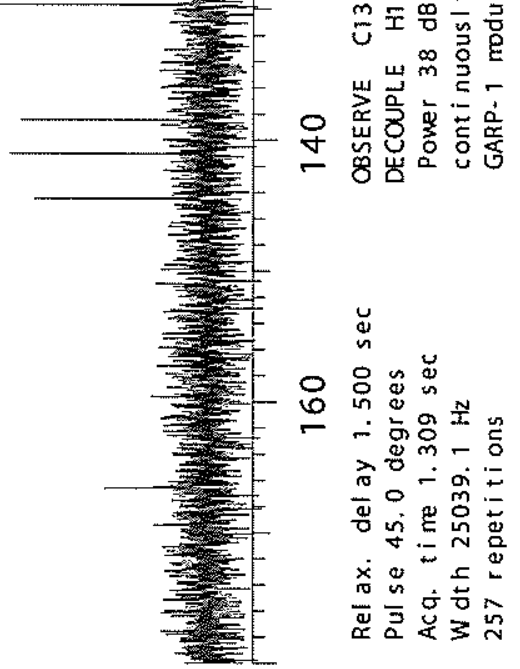




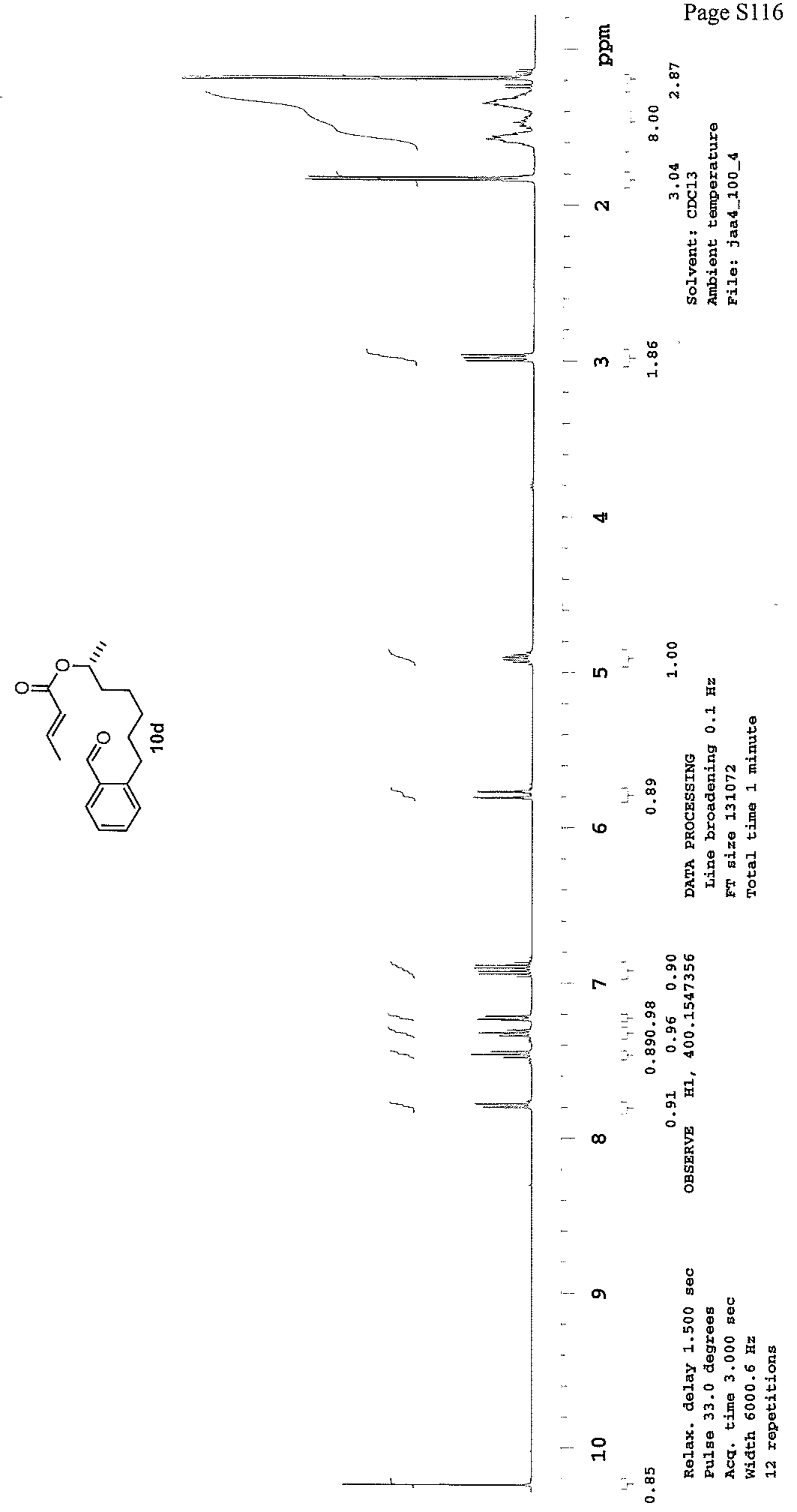


Page S117

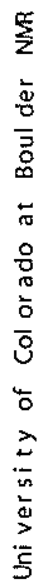

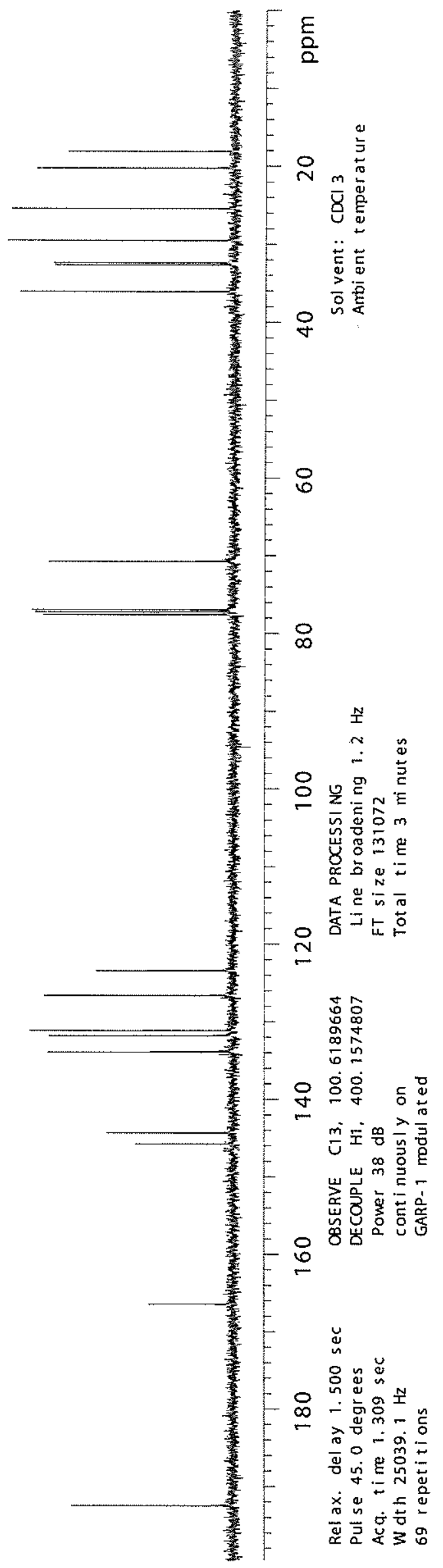



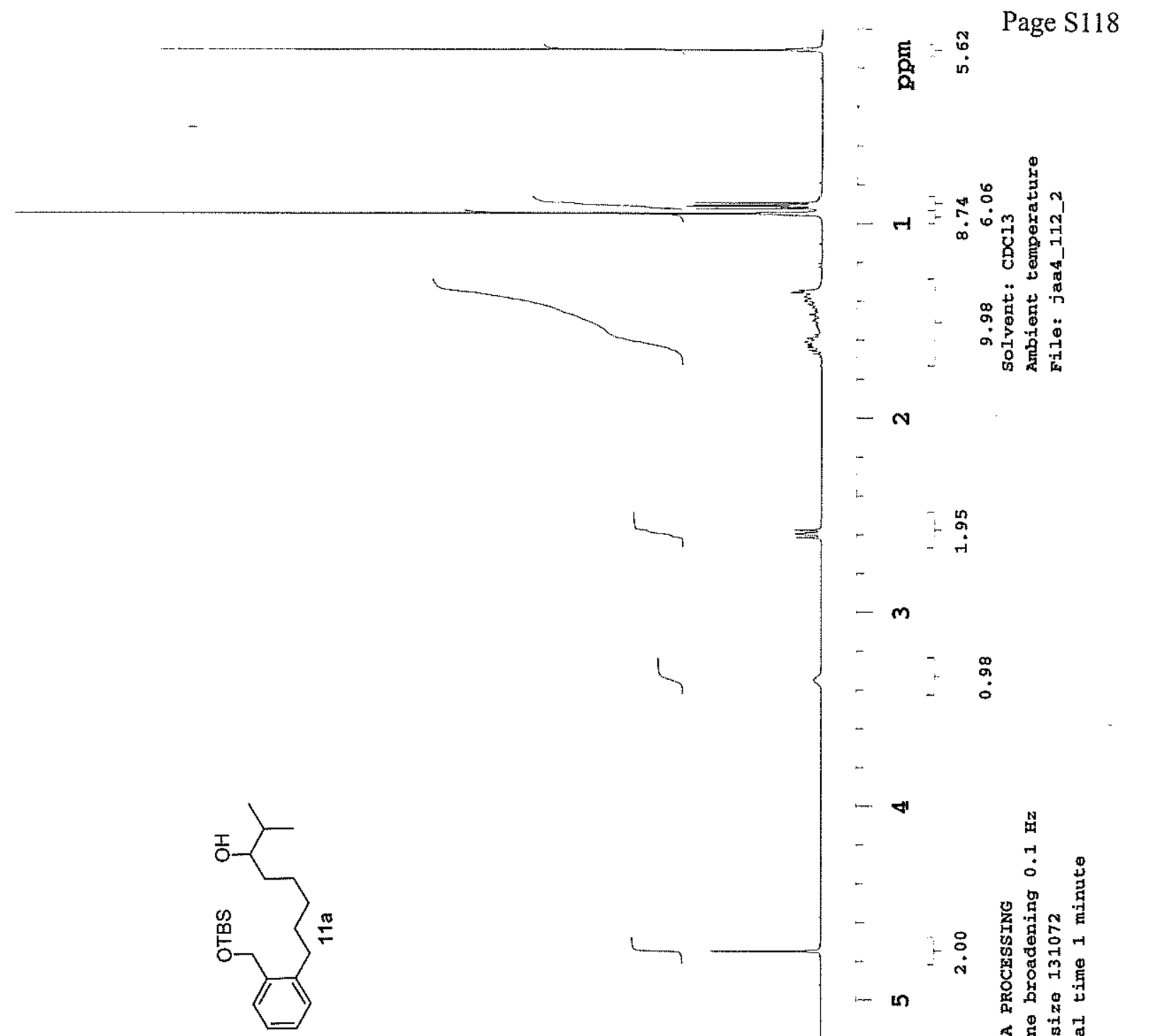
Page S119

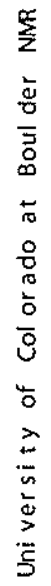
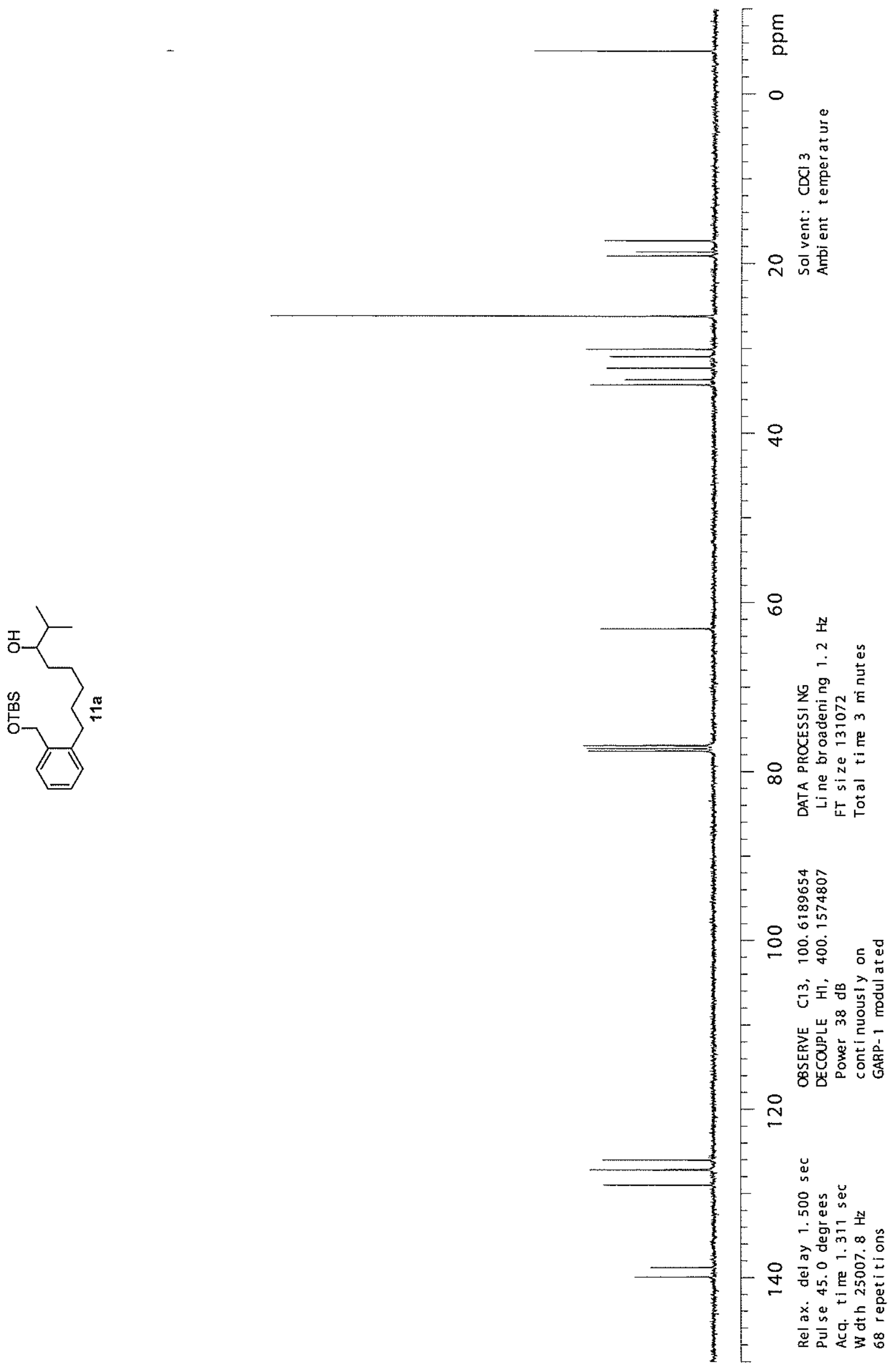


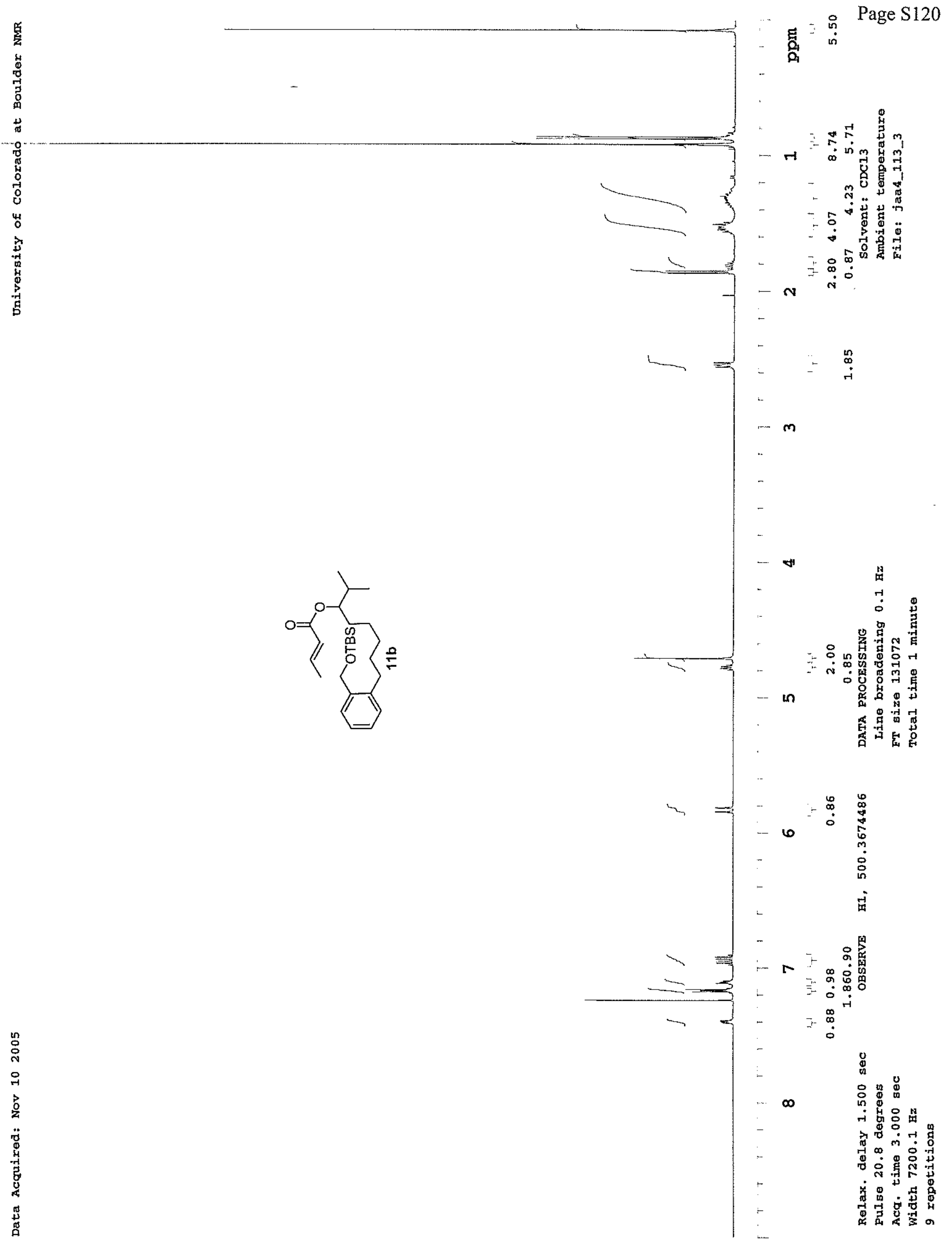


Page S121
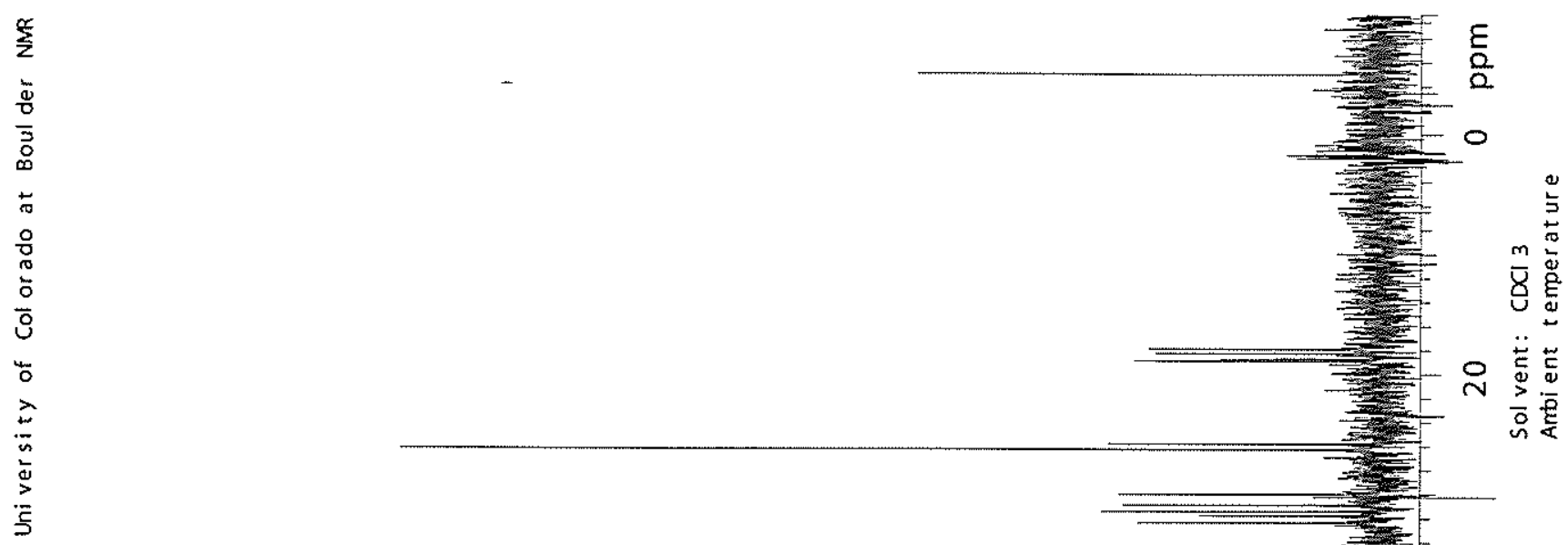

5

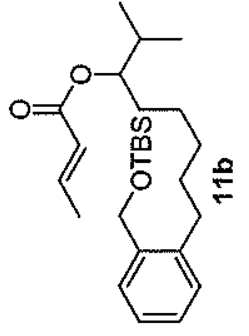

N

$\stackrel{\text { N }}{\stackrel{N}{*}}$

$\infty$ 至

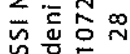

嵌

范嵒

䟘六

客要占

8

50
00
0
0
5
5
0

官导

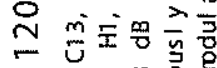

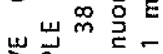

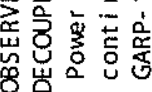

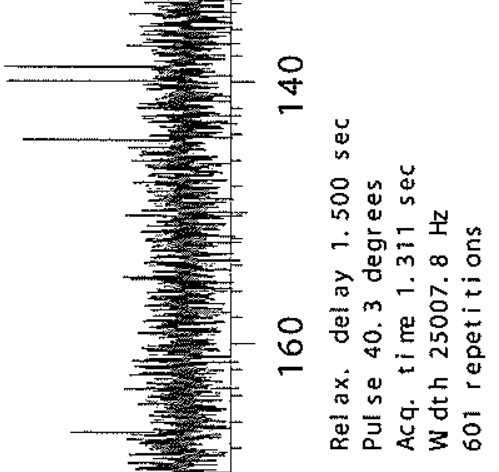




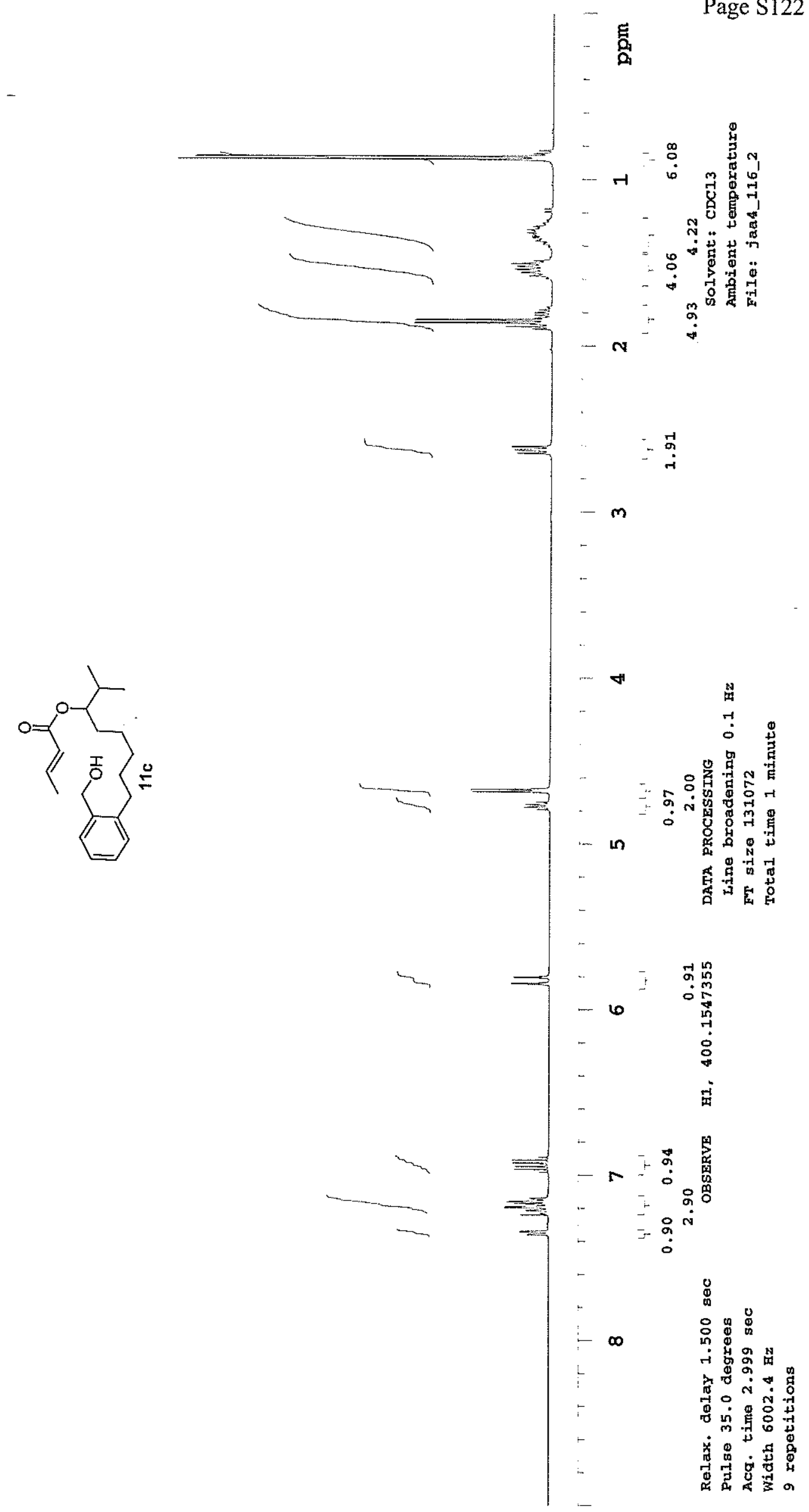


Page S123

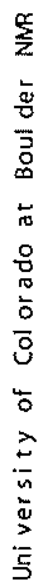

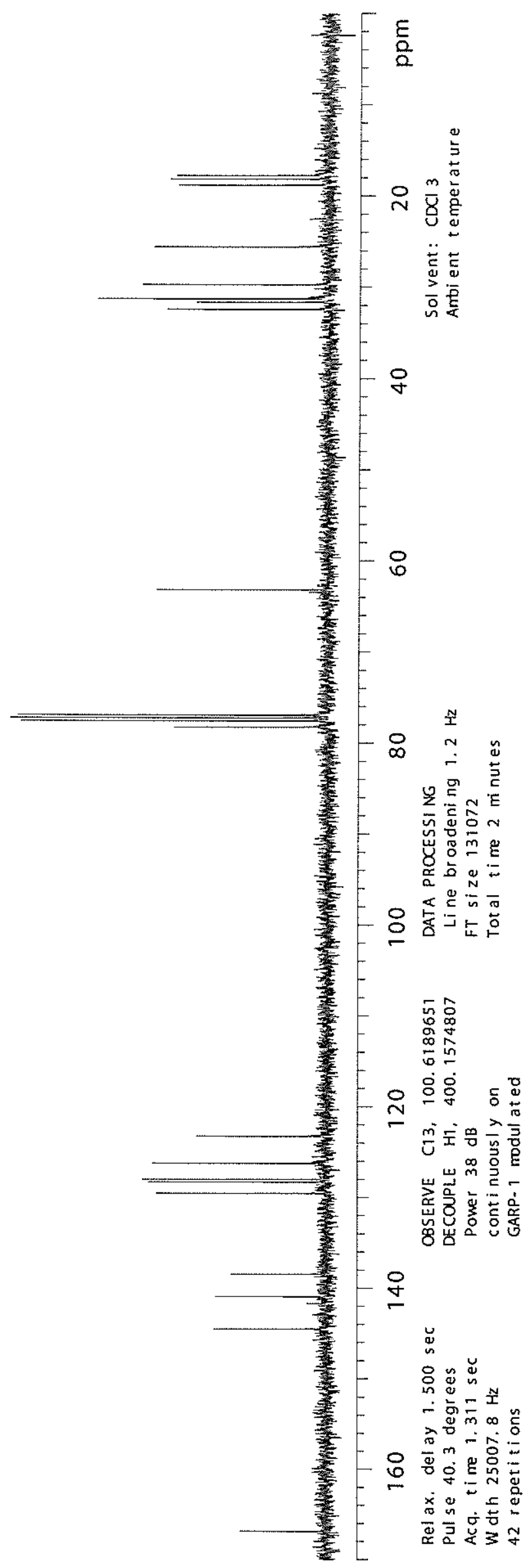




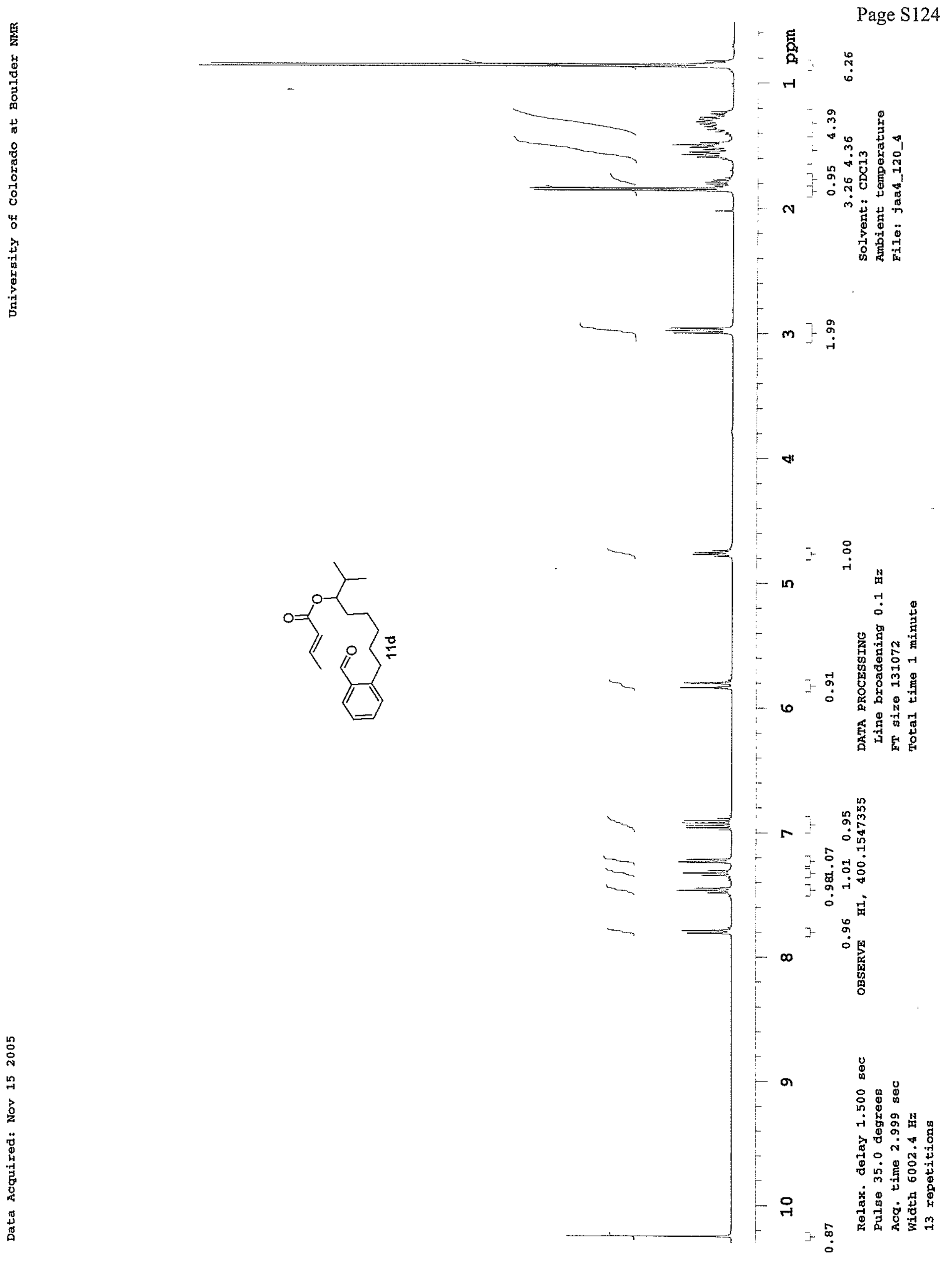




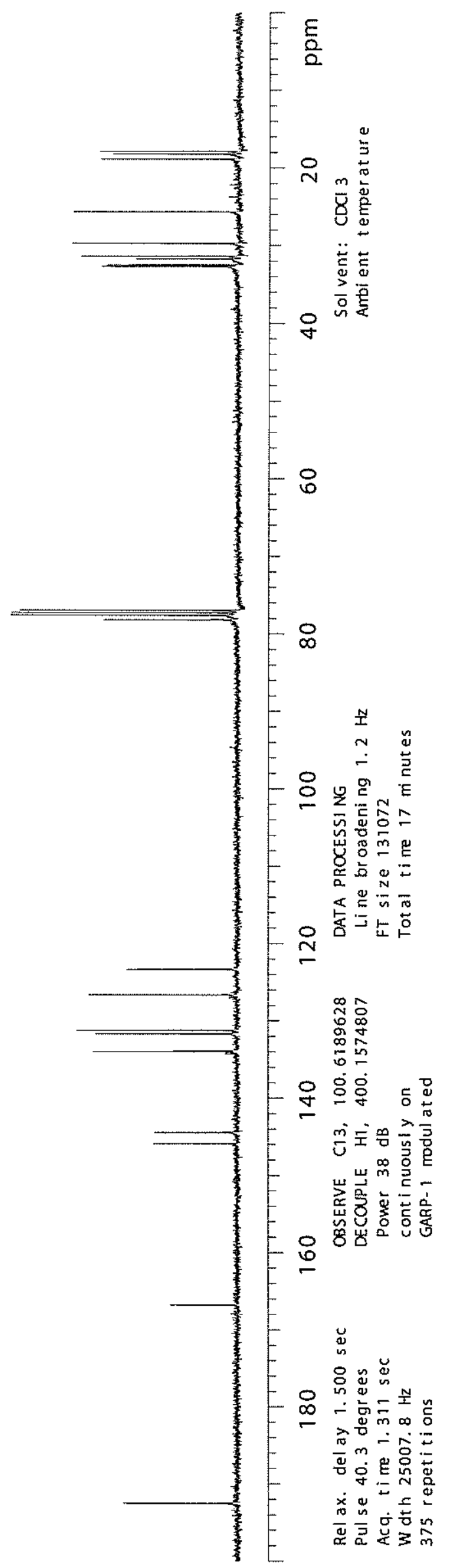




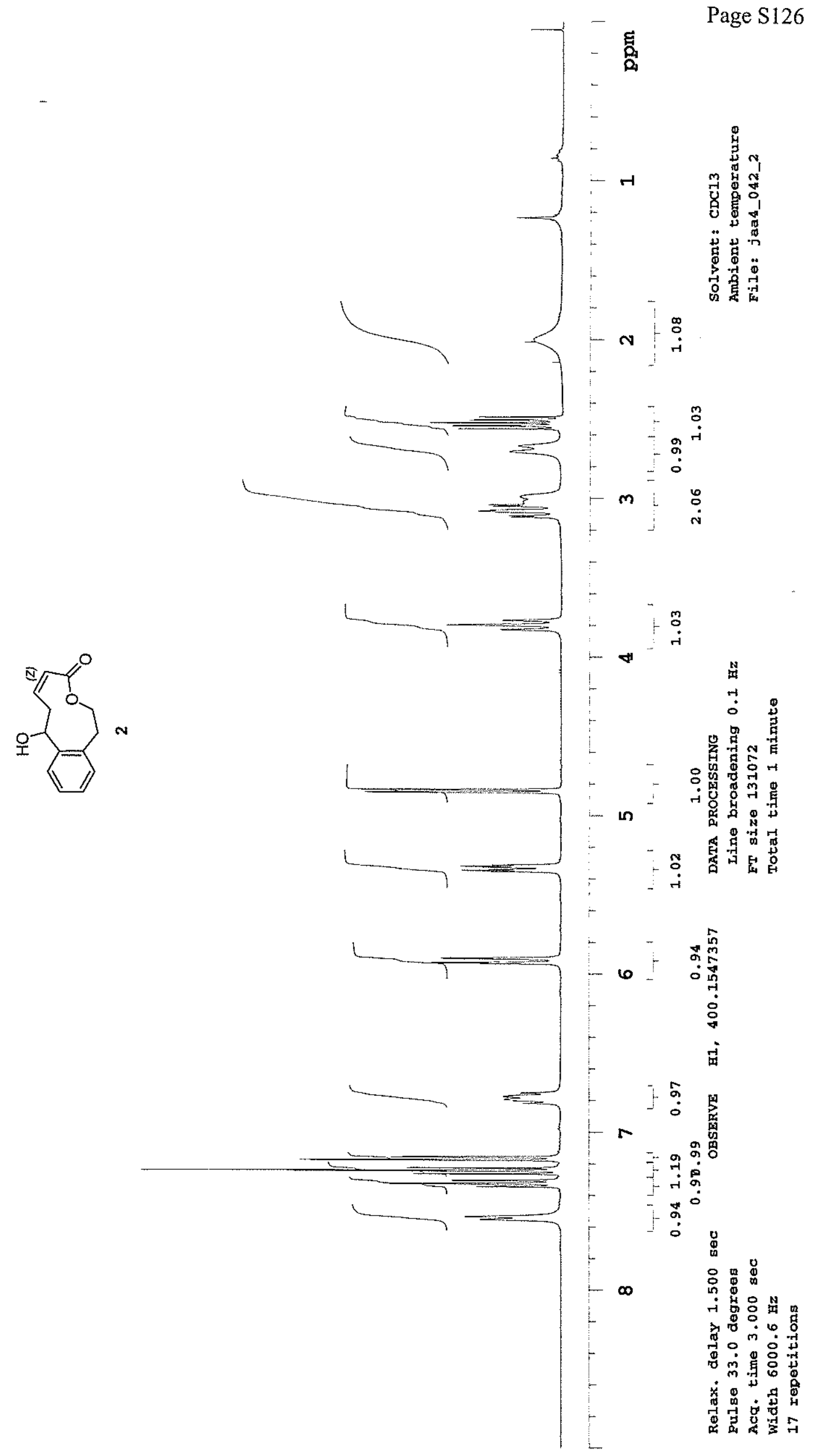



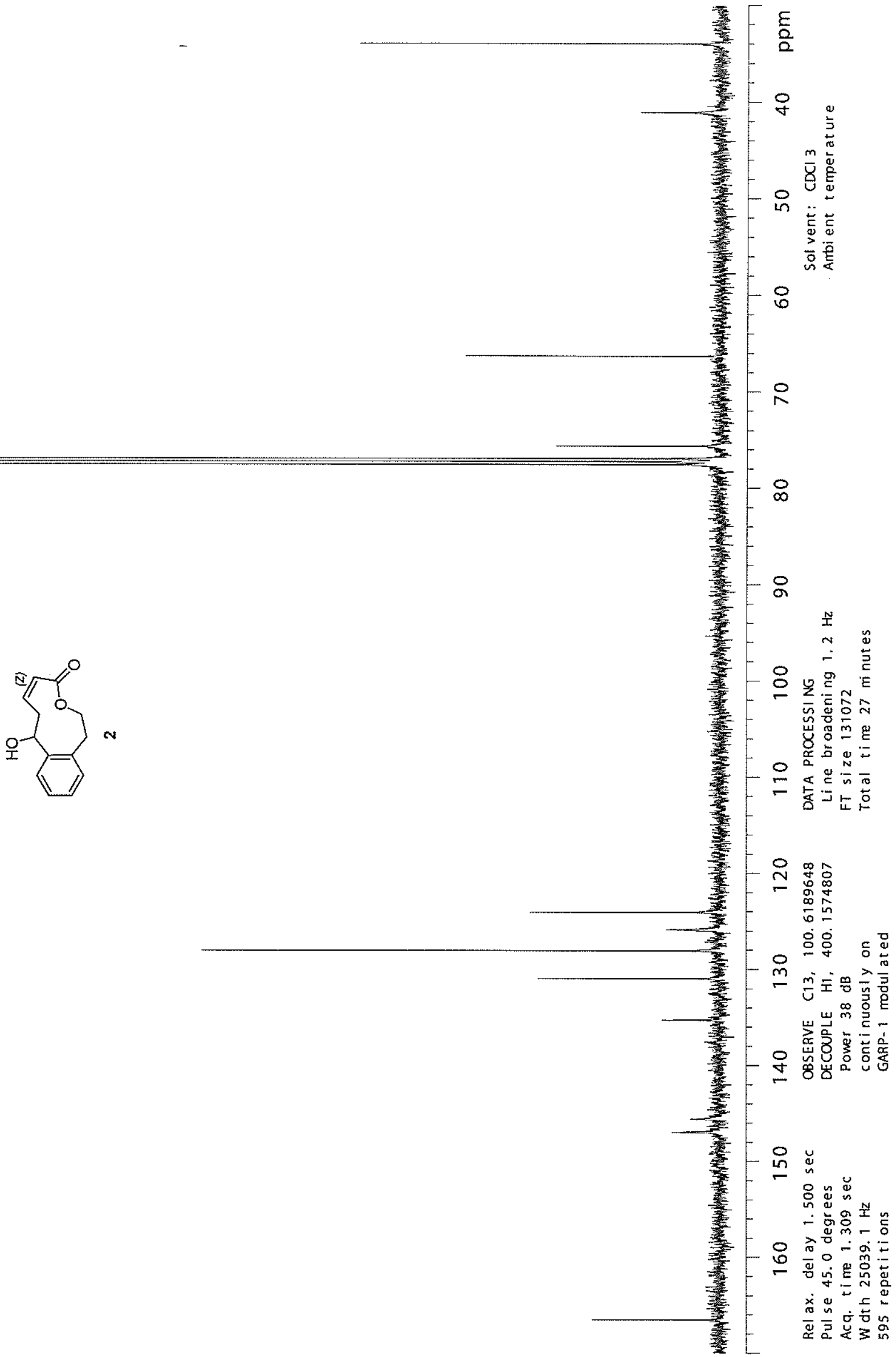


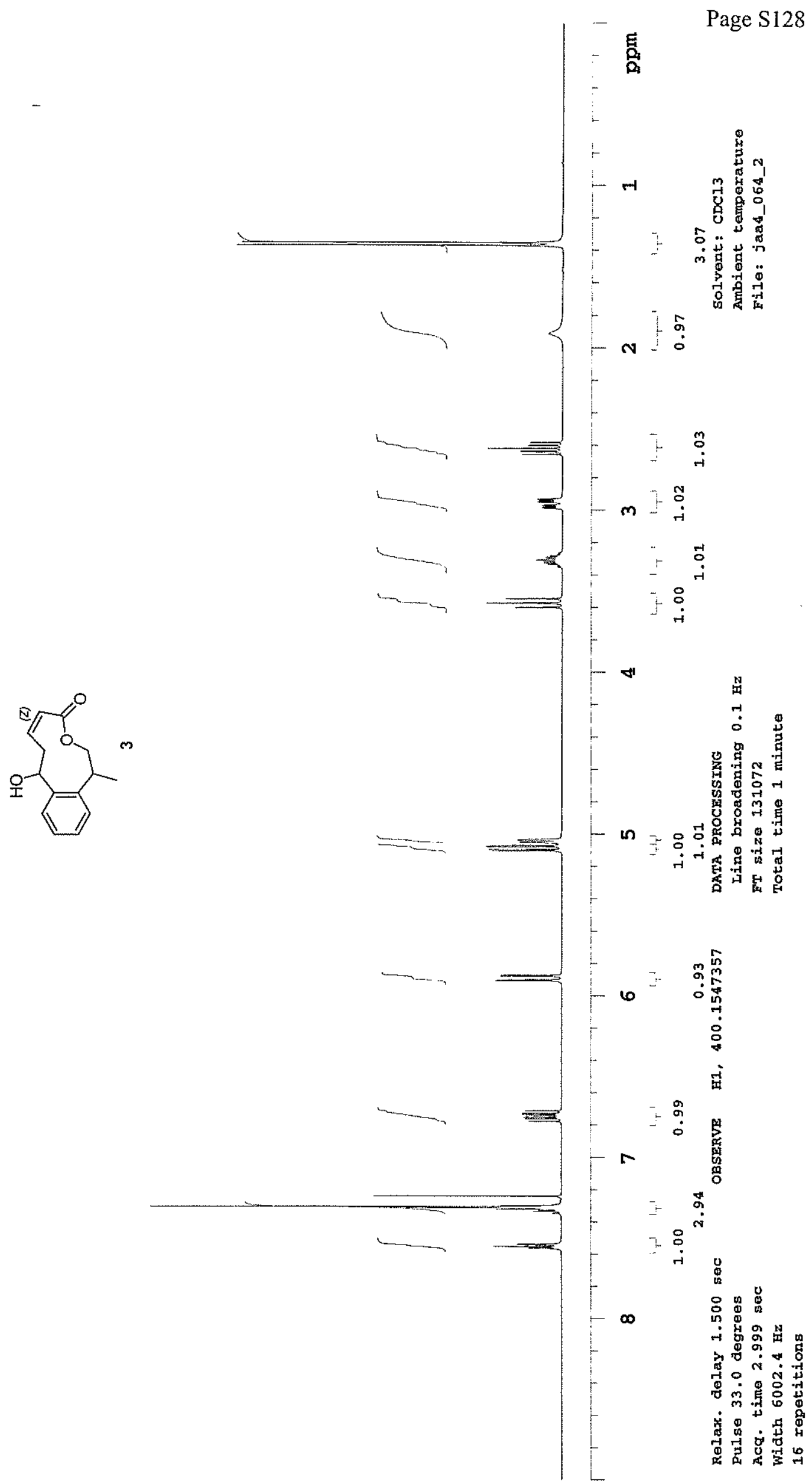




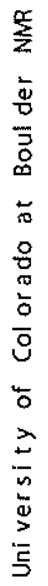

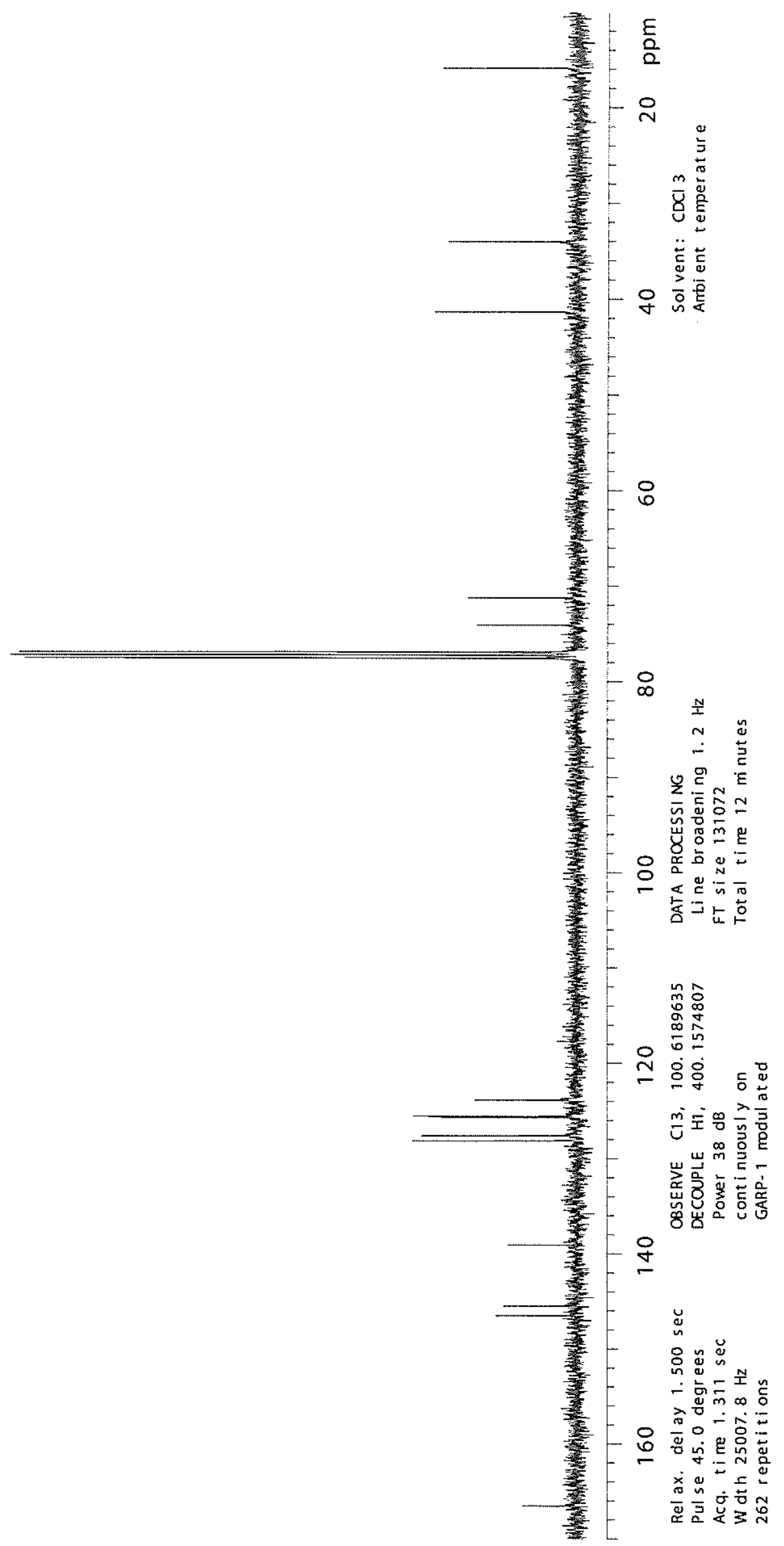




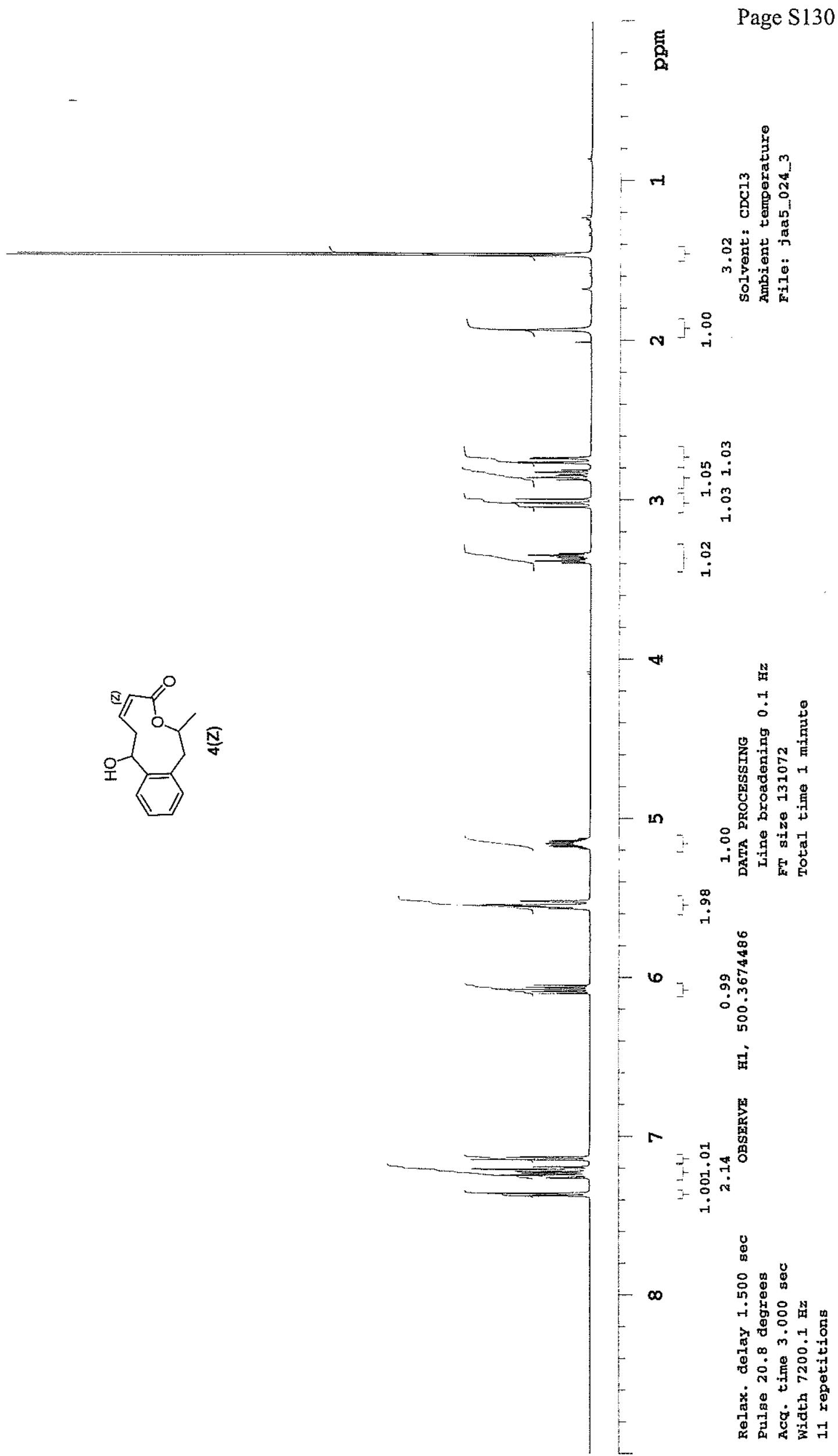




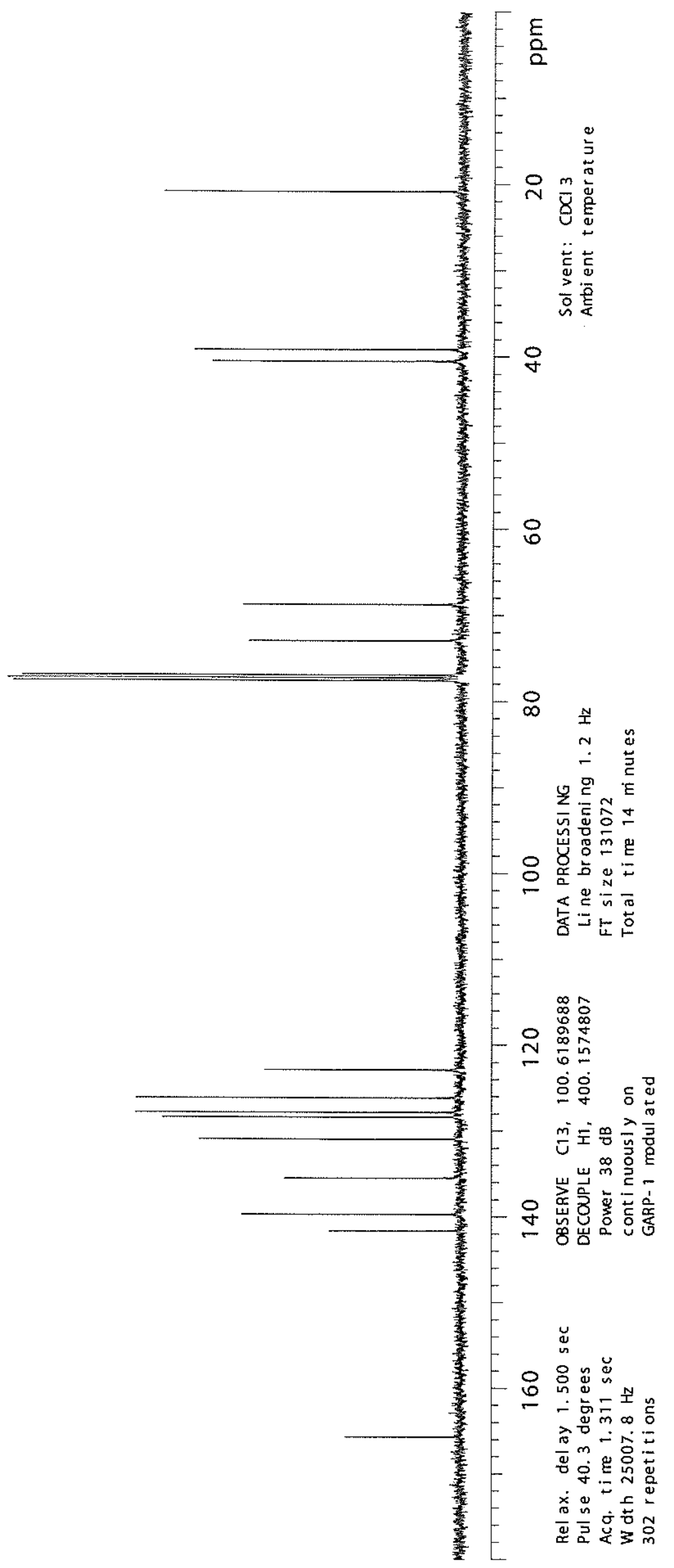



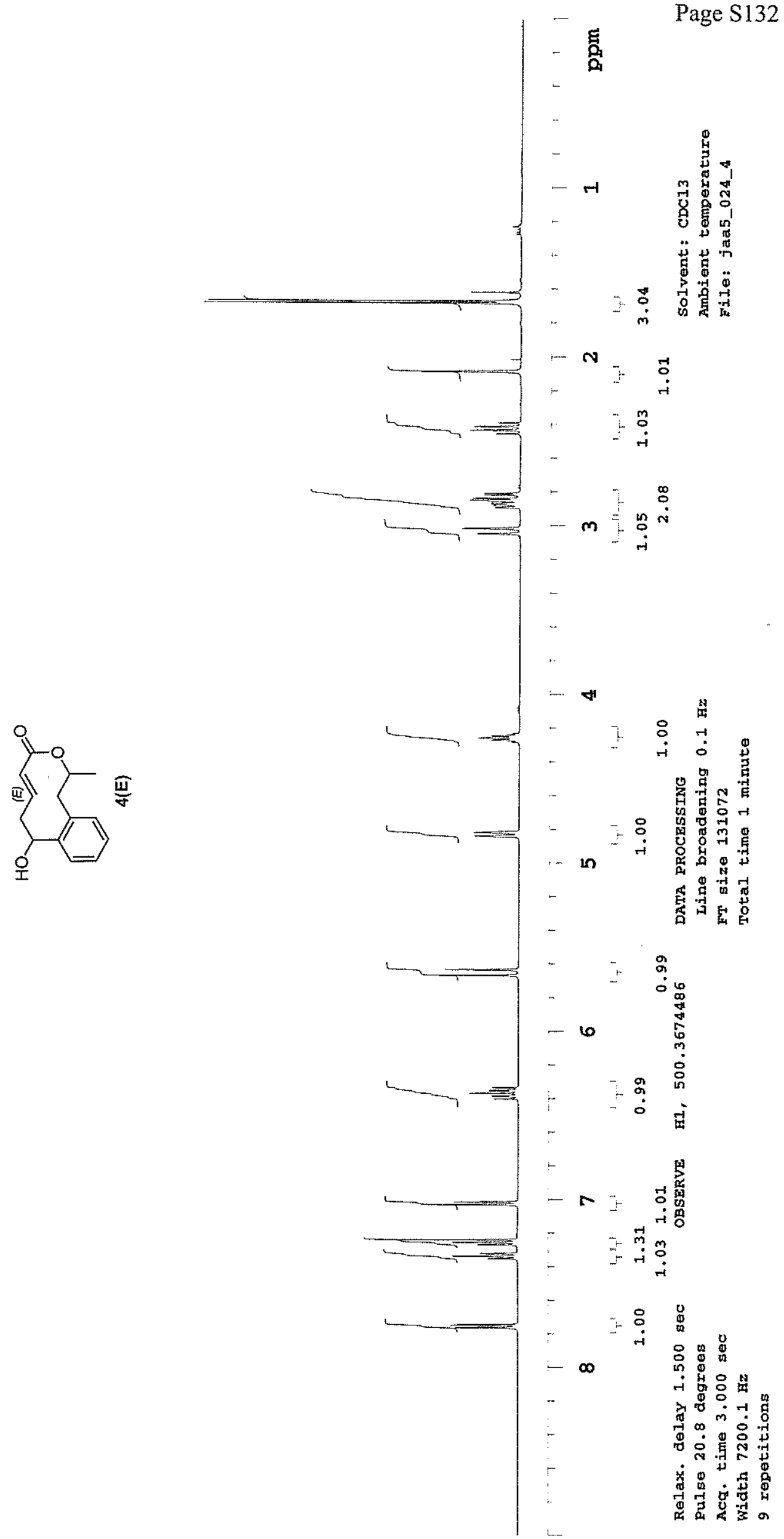
Page S133

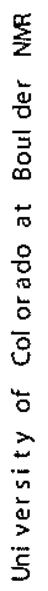

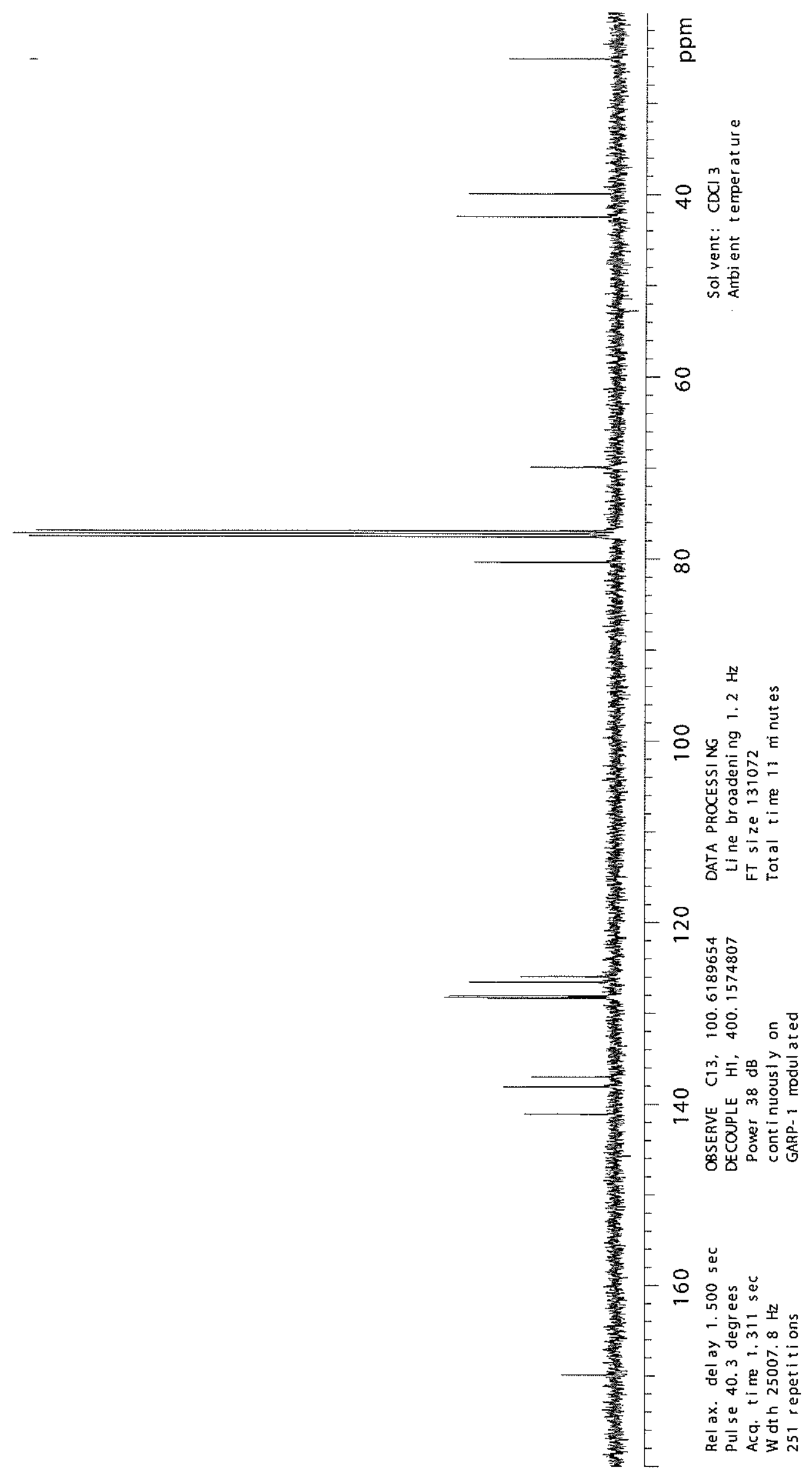




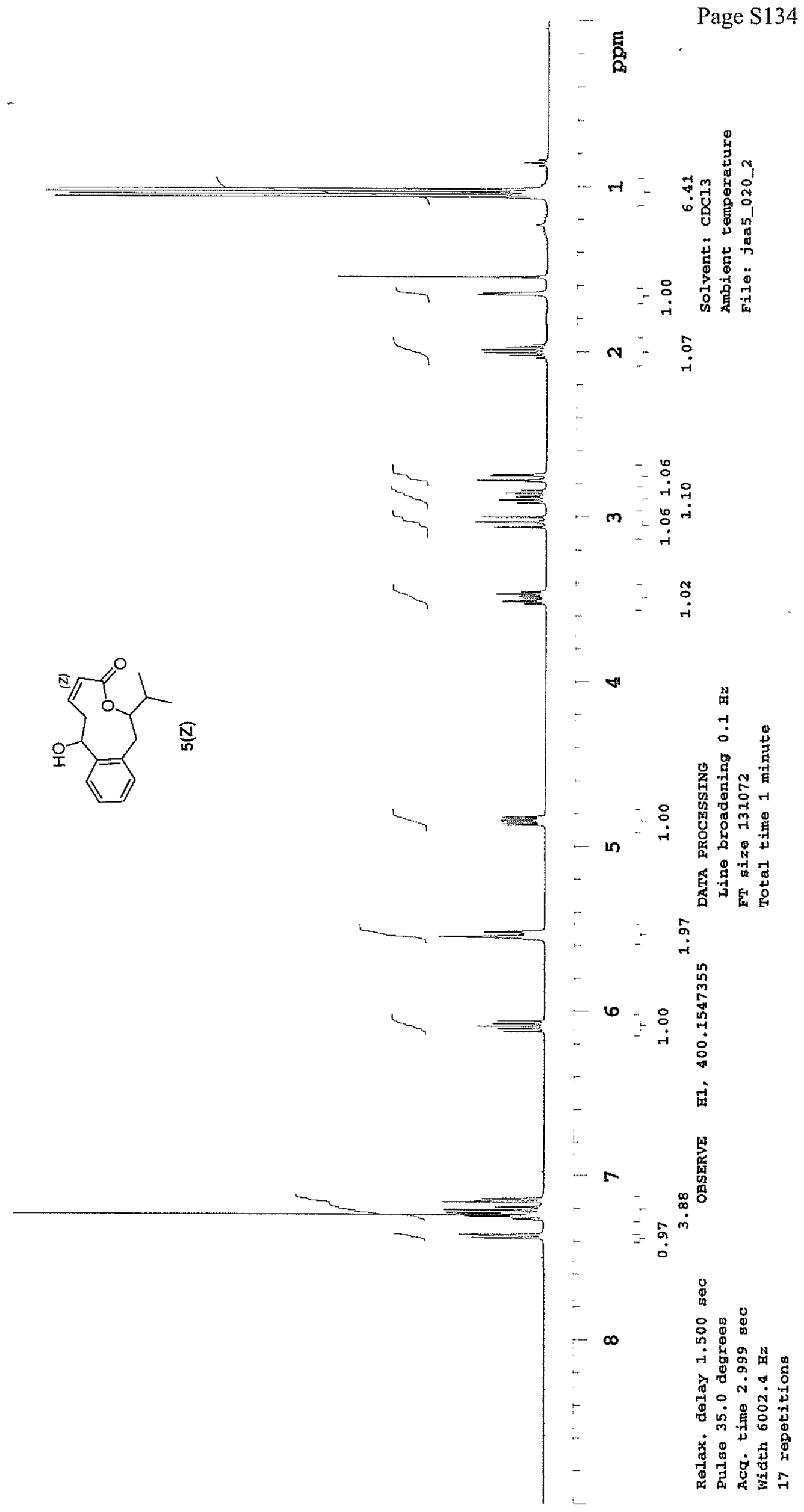


Page S135

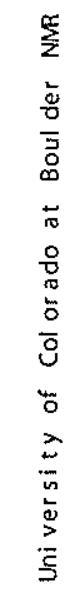

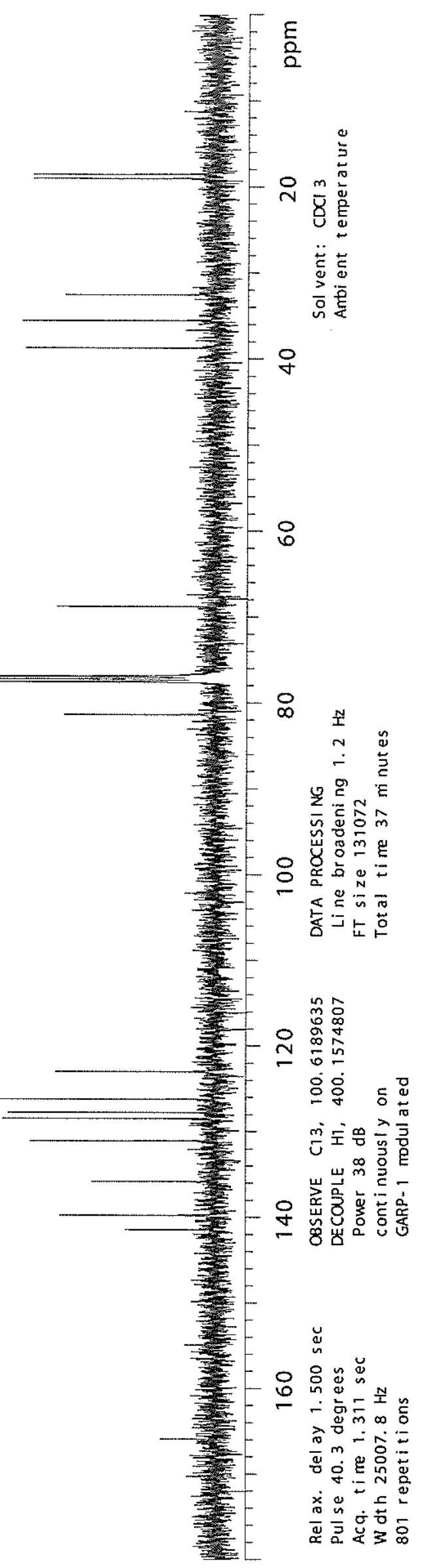




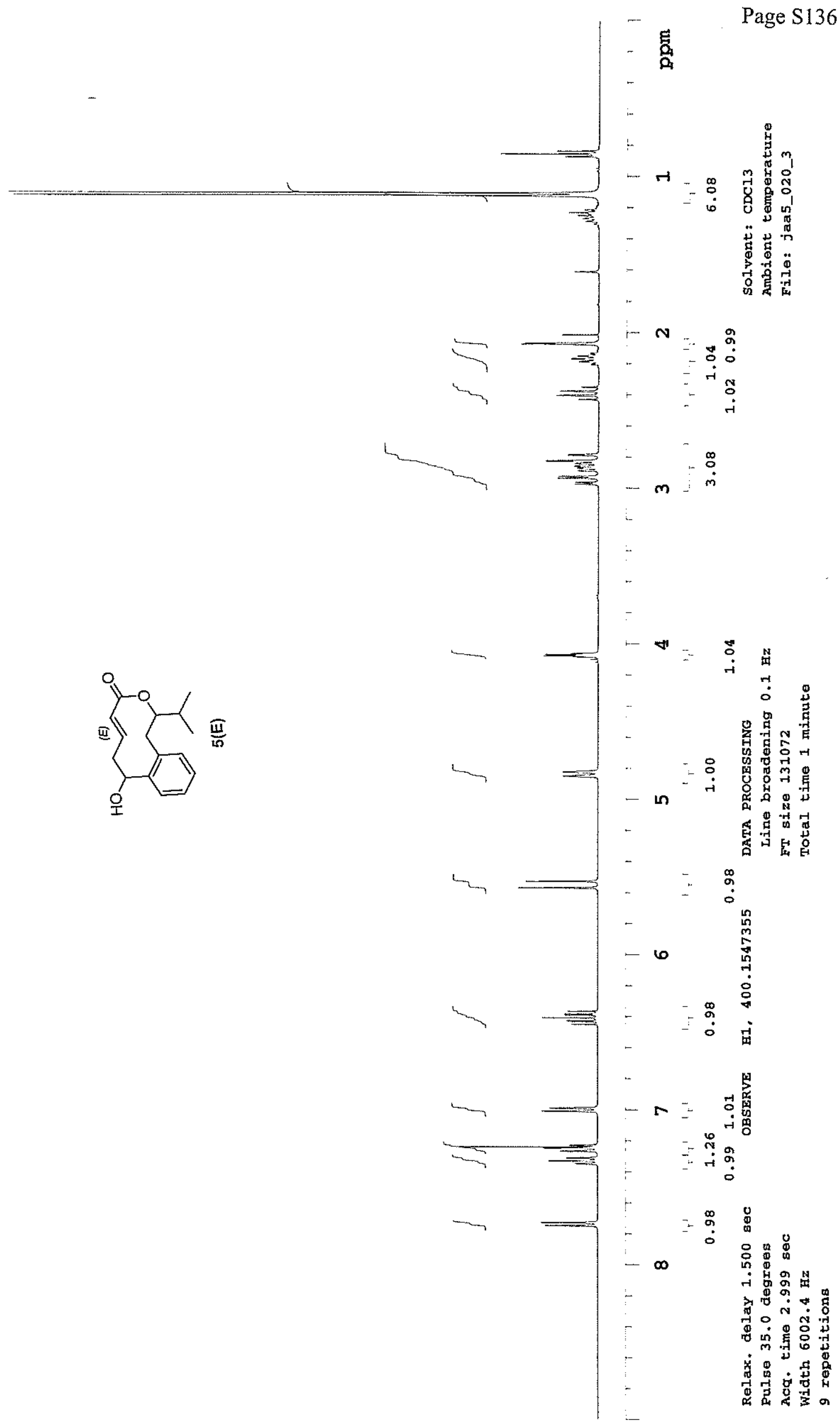




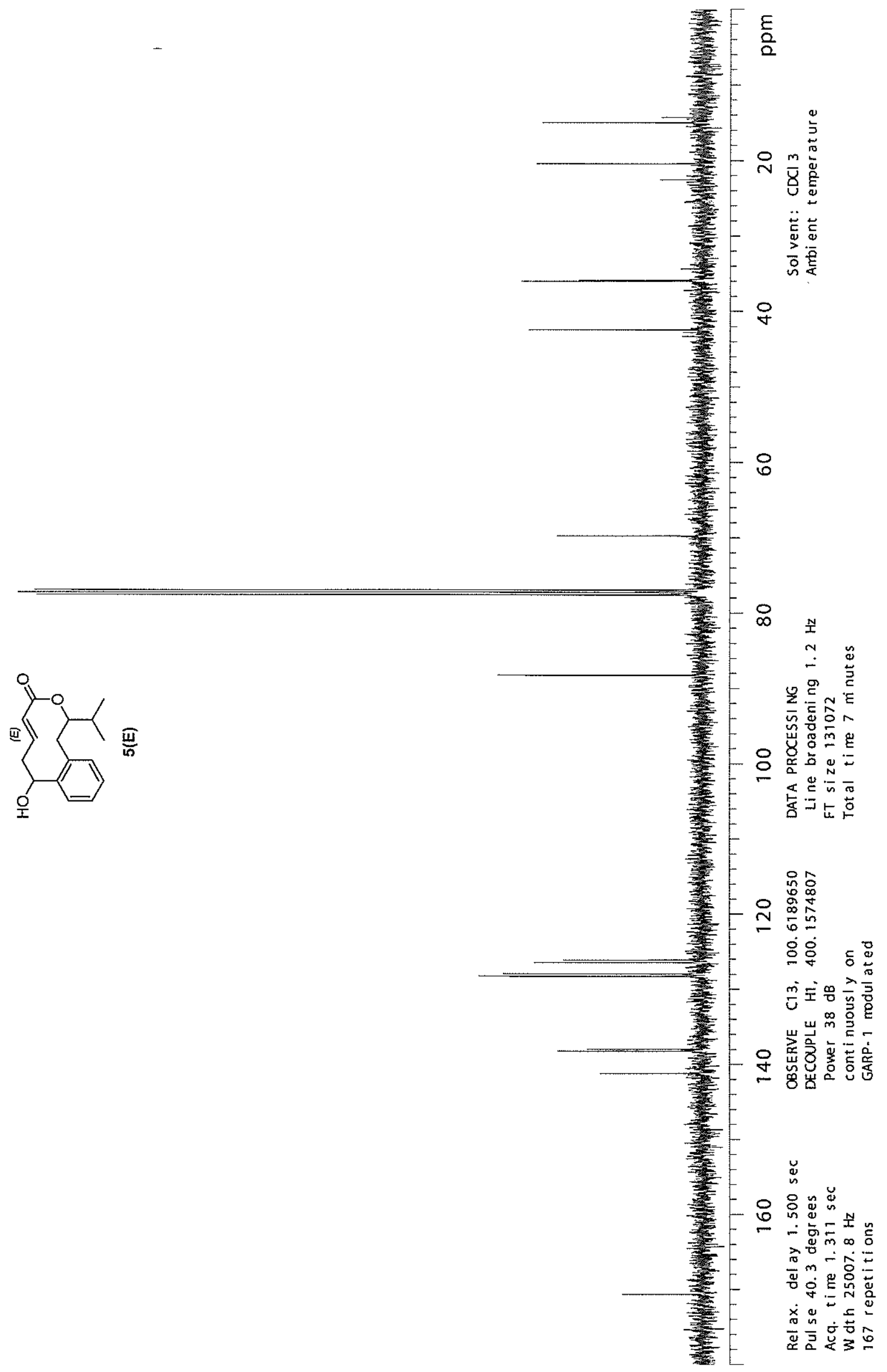




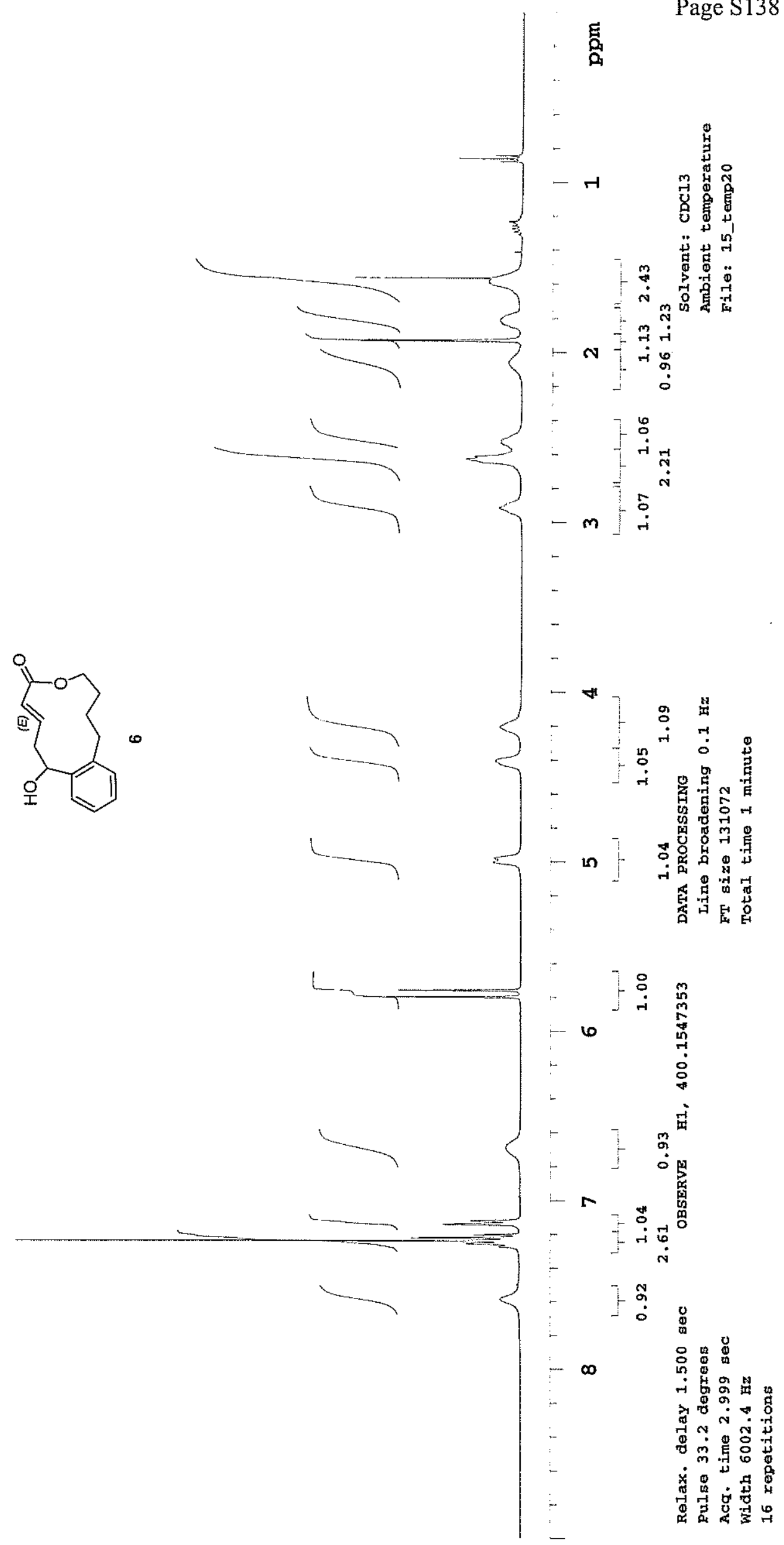



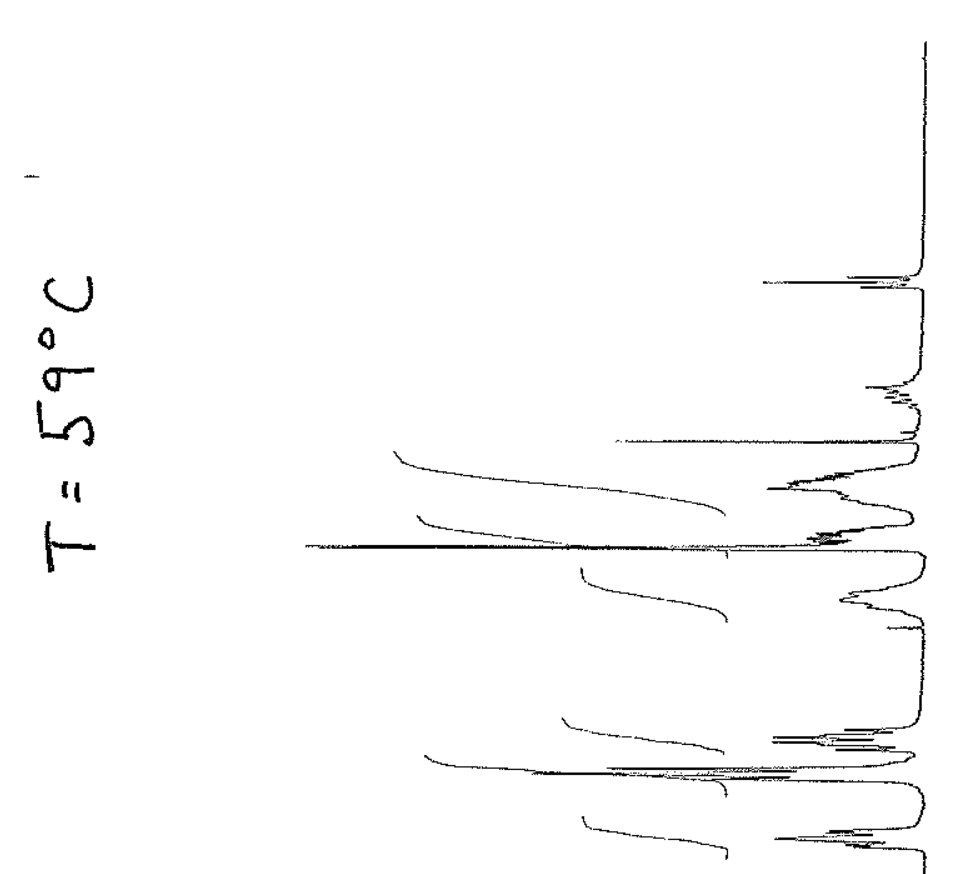

Page S139
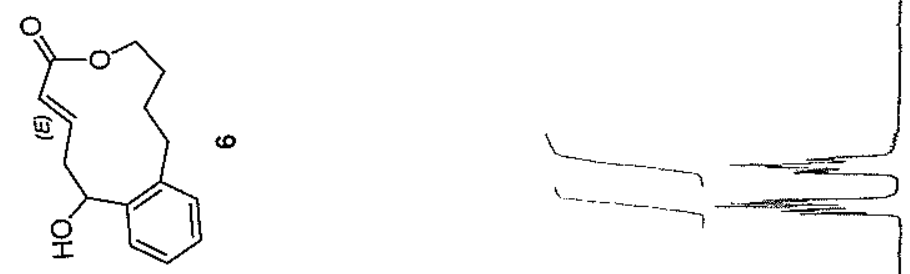

$-\forall$

I
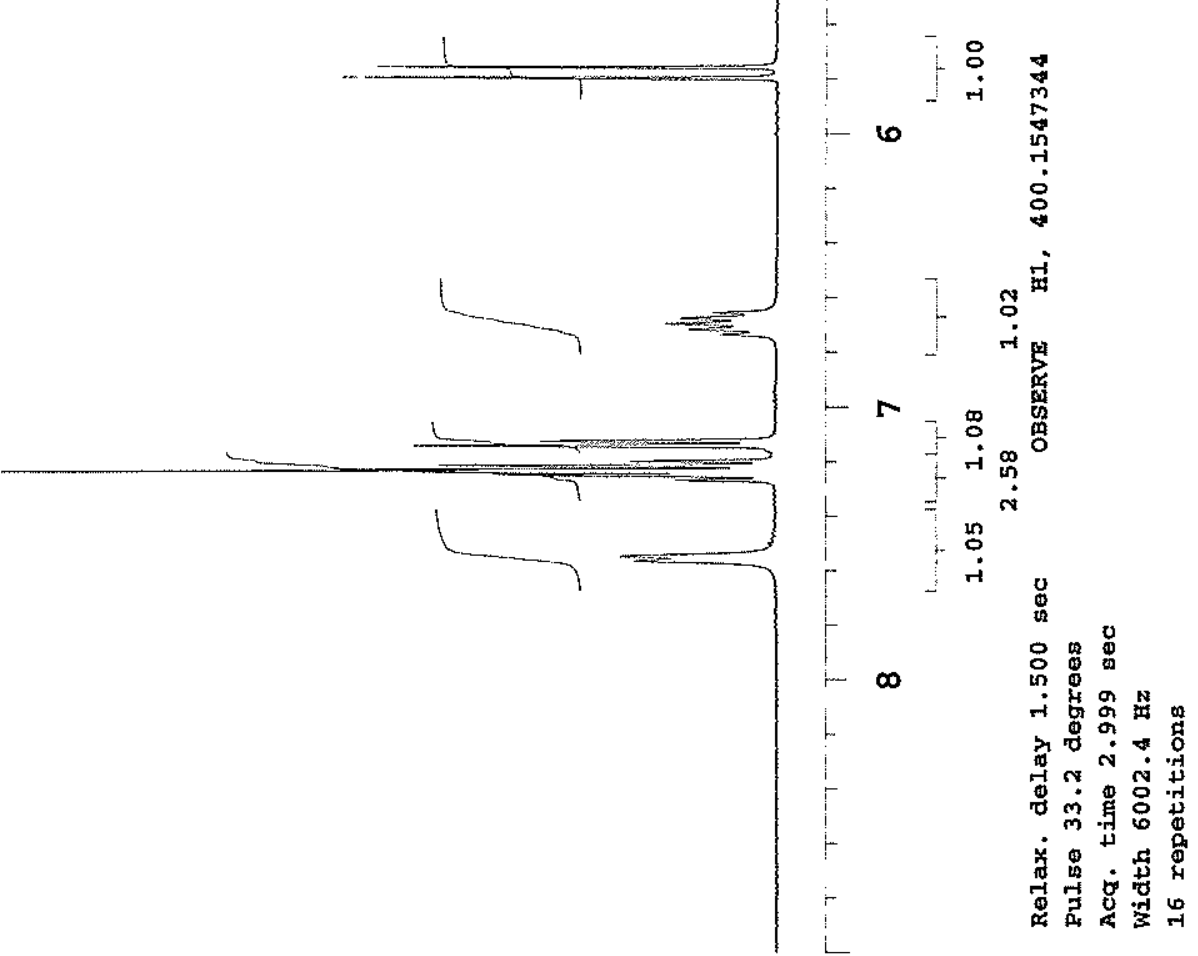
Page $\$ 140$

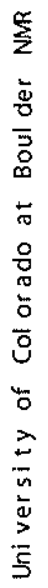

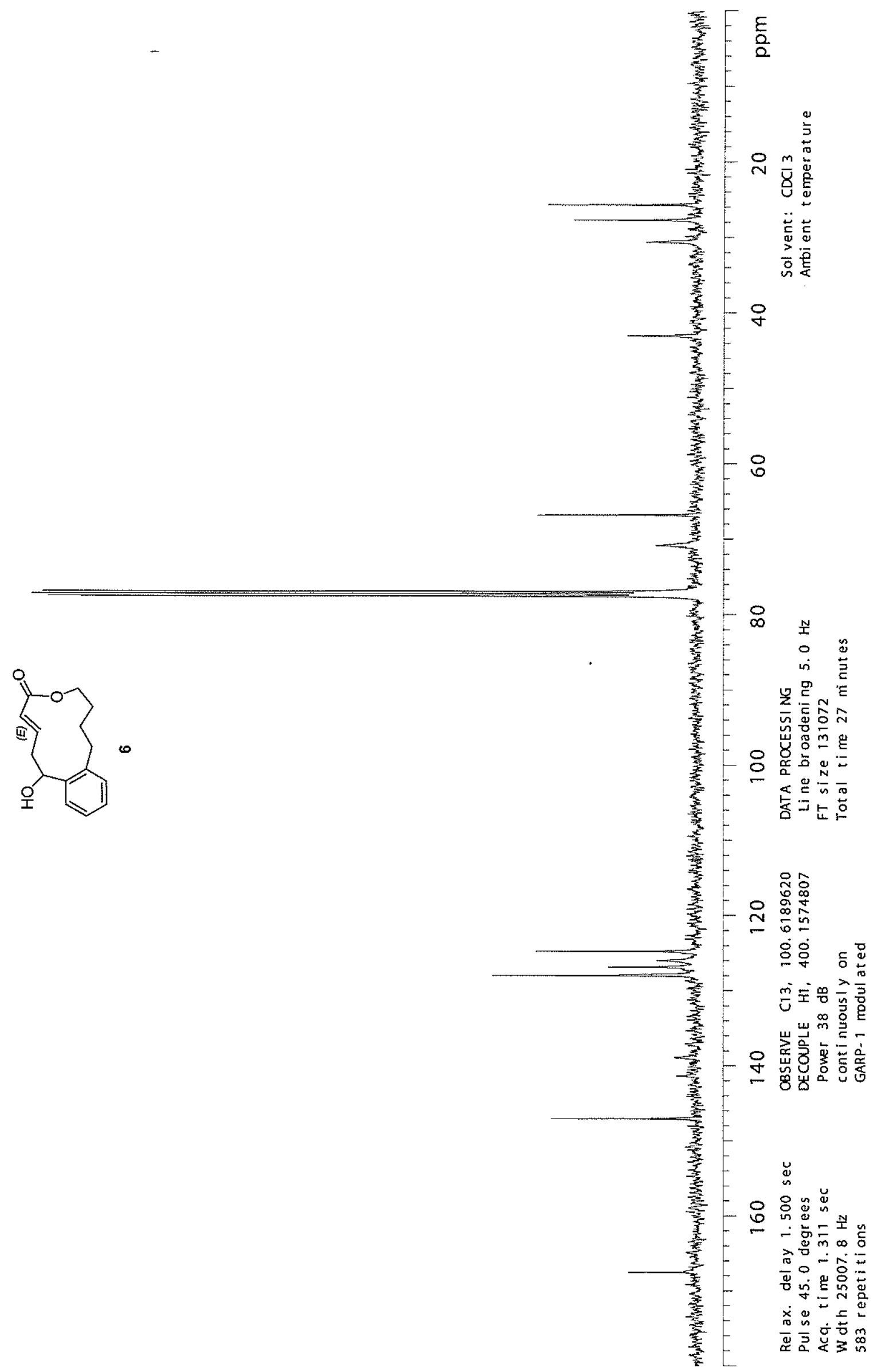




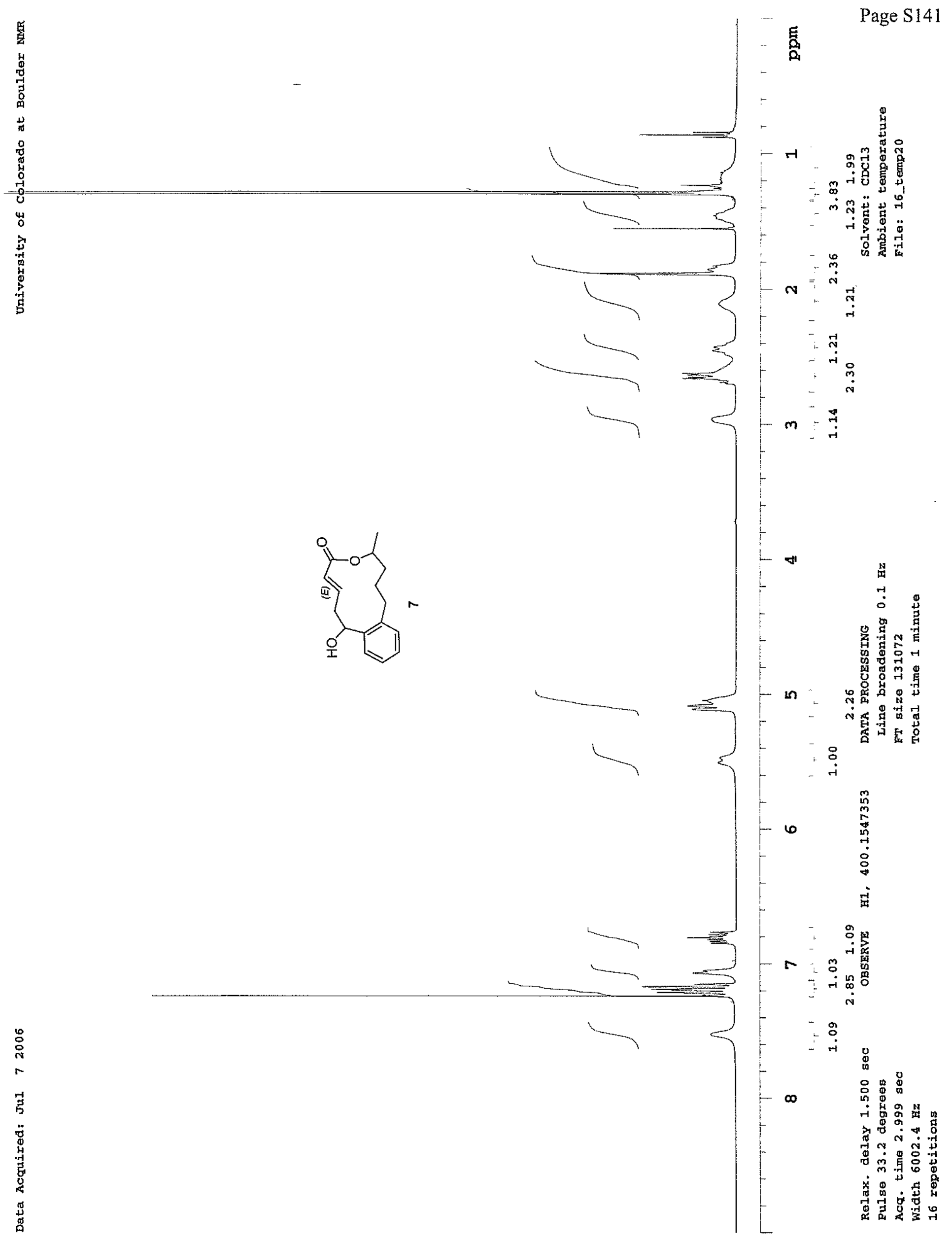




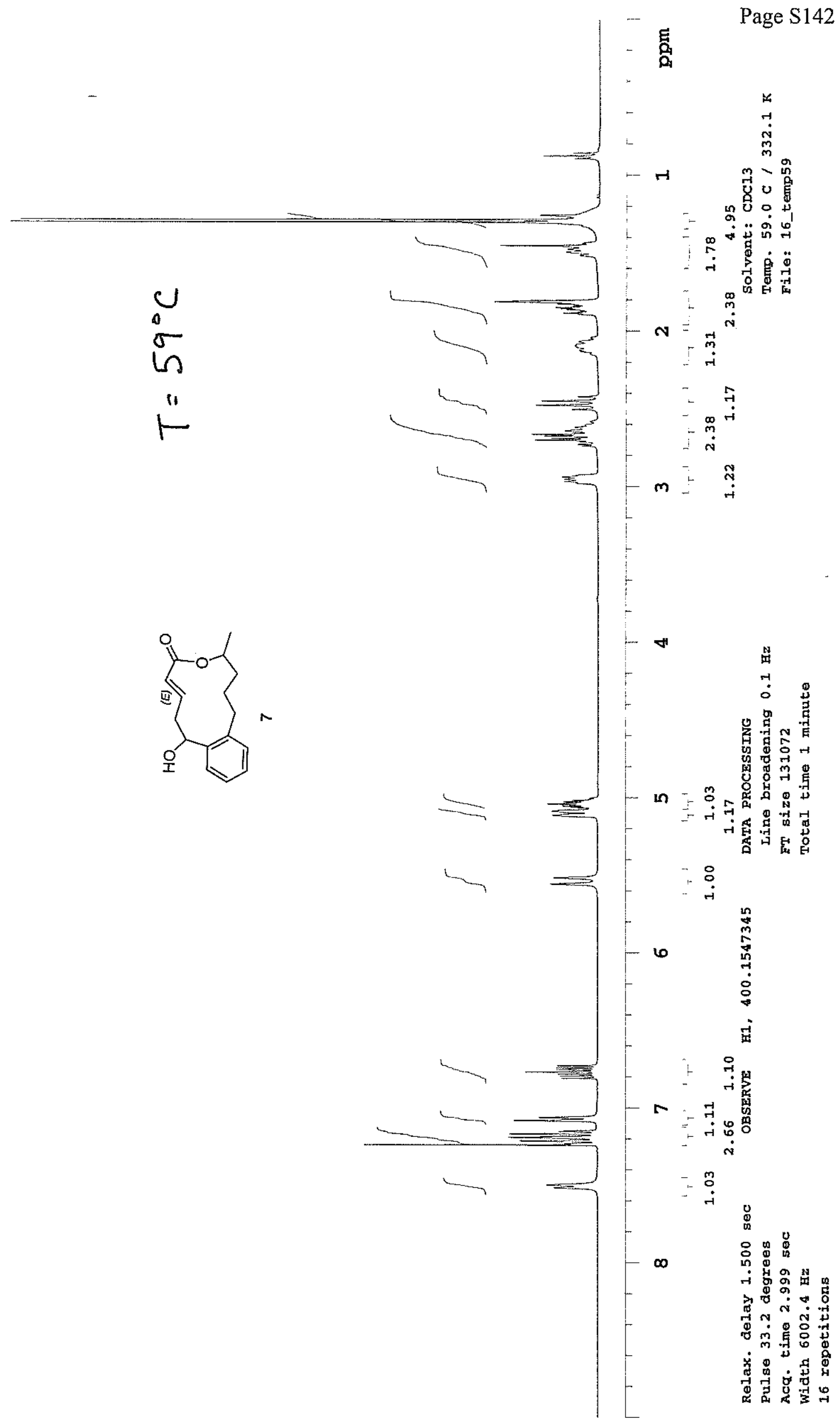


Page S143

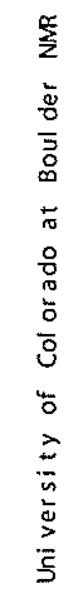

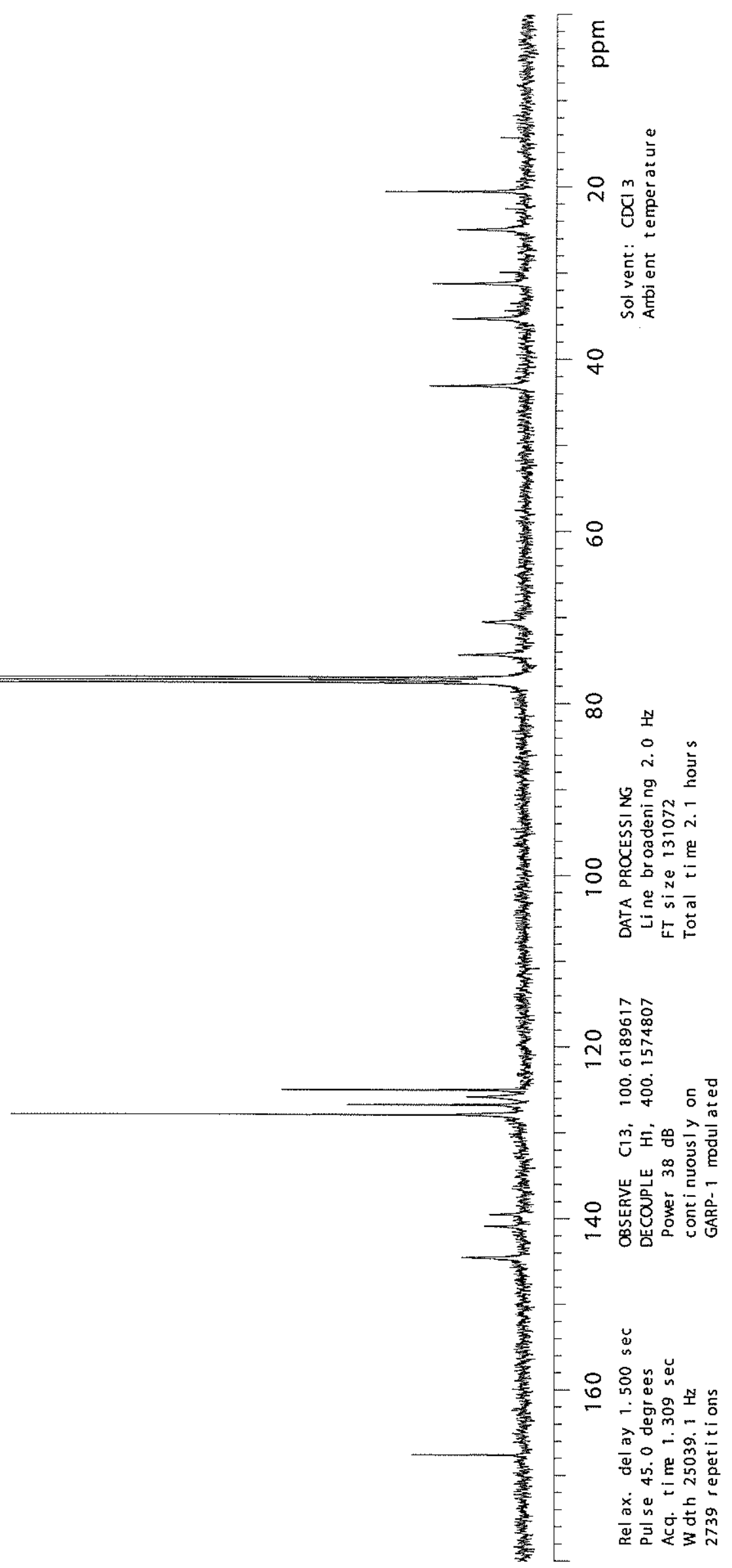




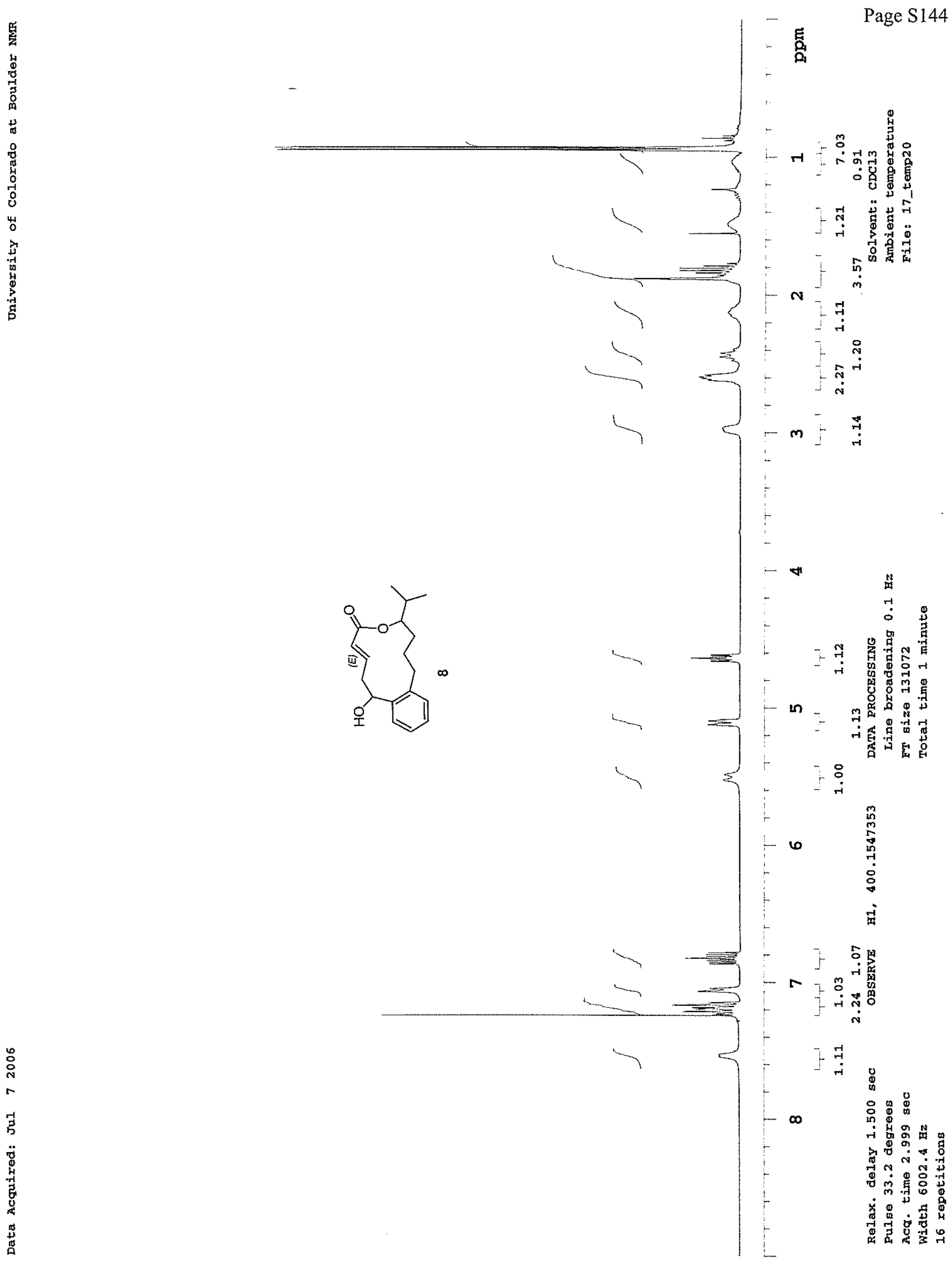



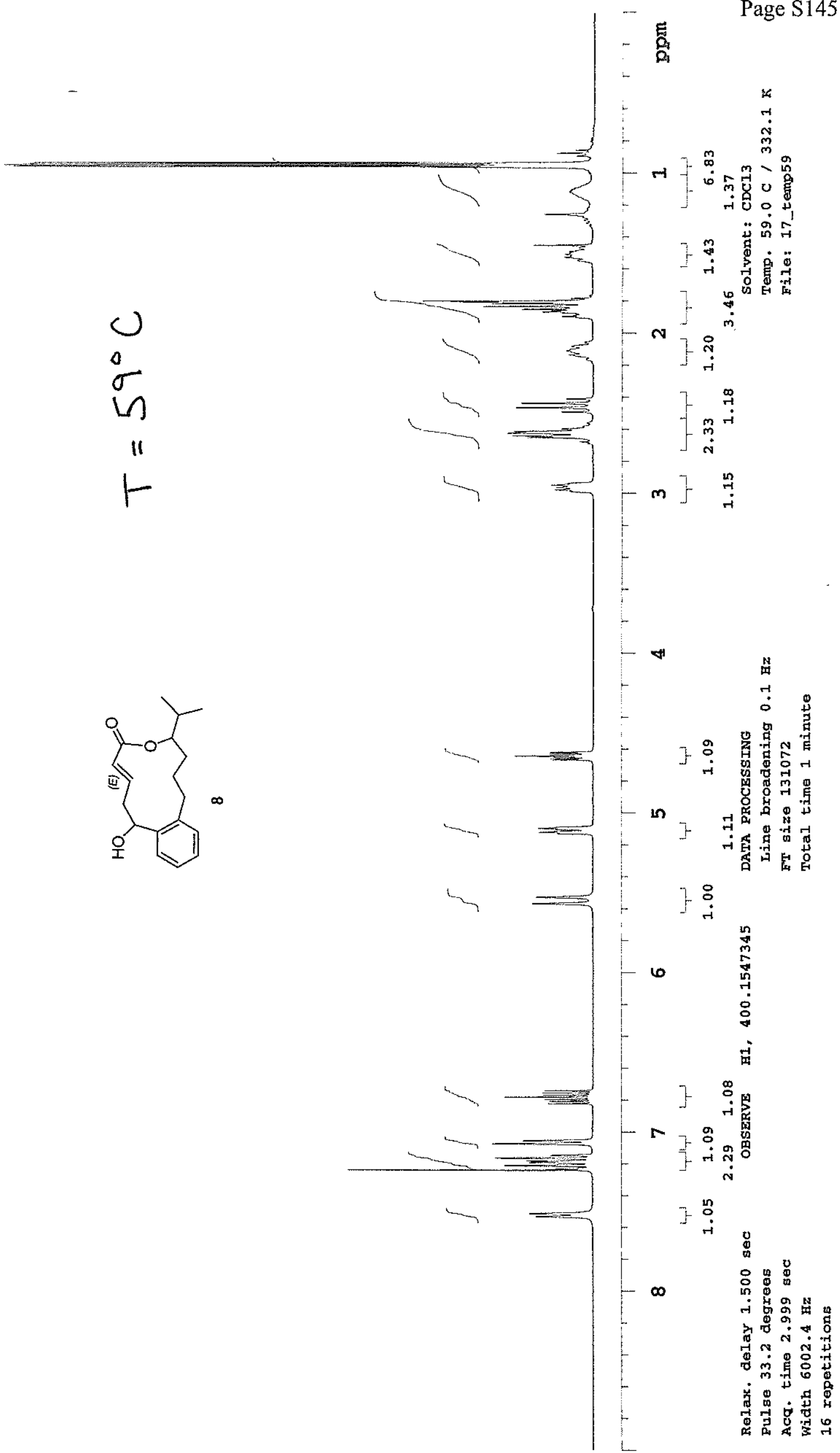
<smiles>C/C=C/C(=O)OC(CCCc1ccccc1C(C)O)C(C)C</smiles>

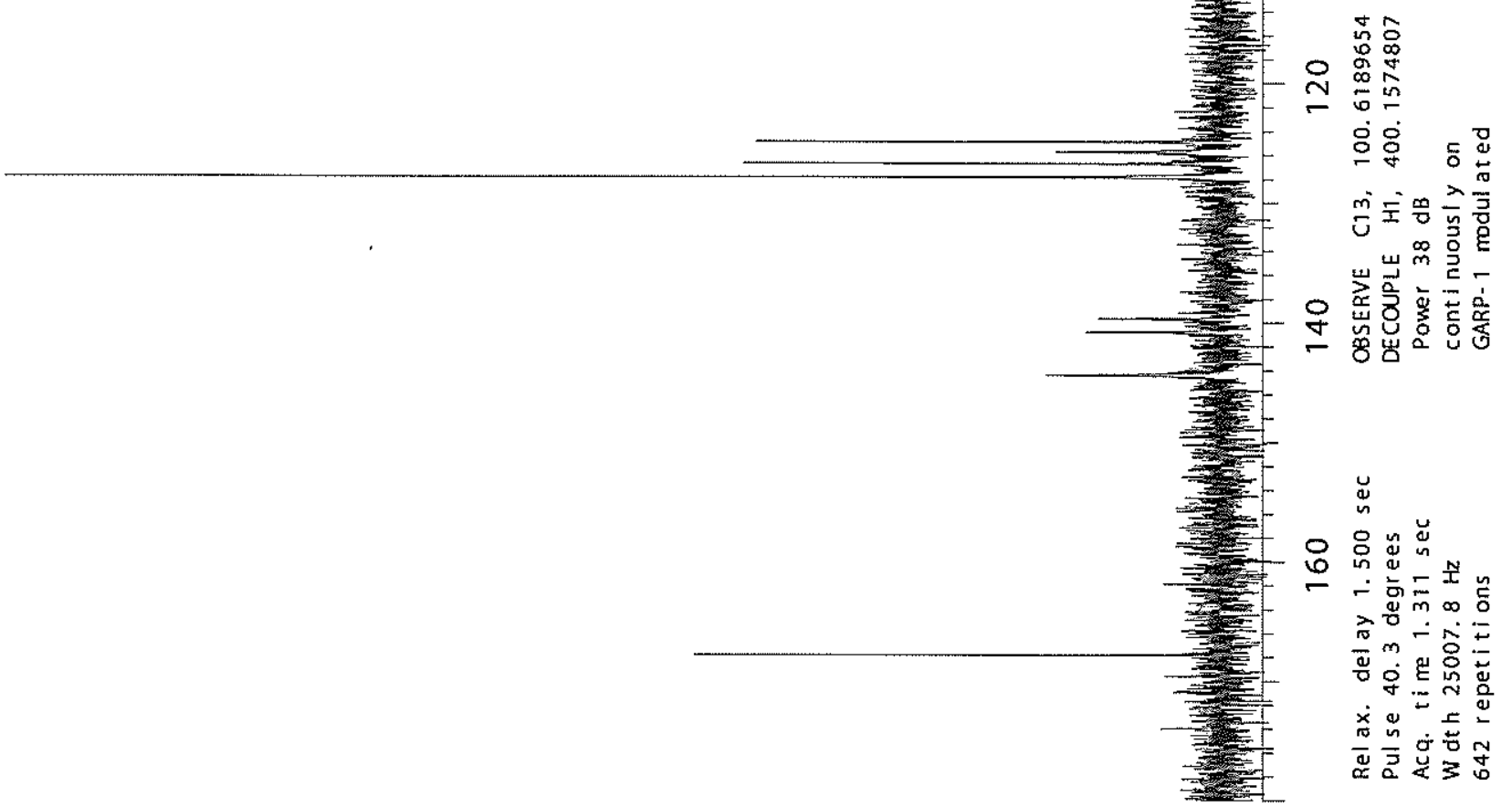




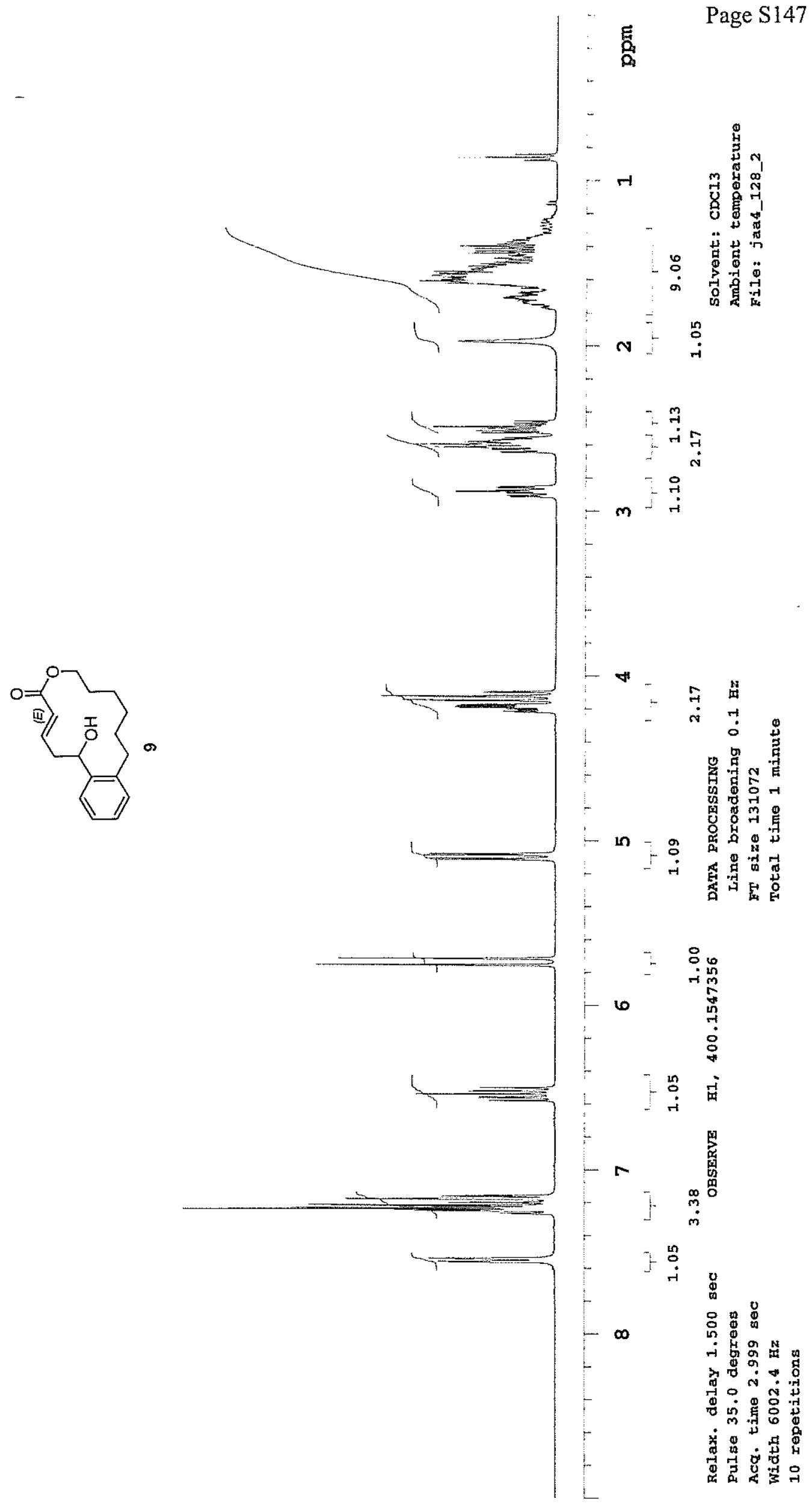


Page S148

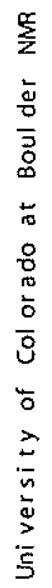

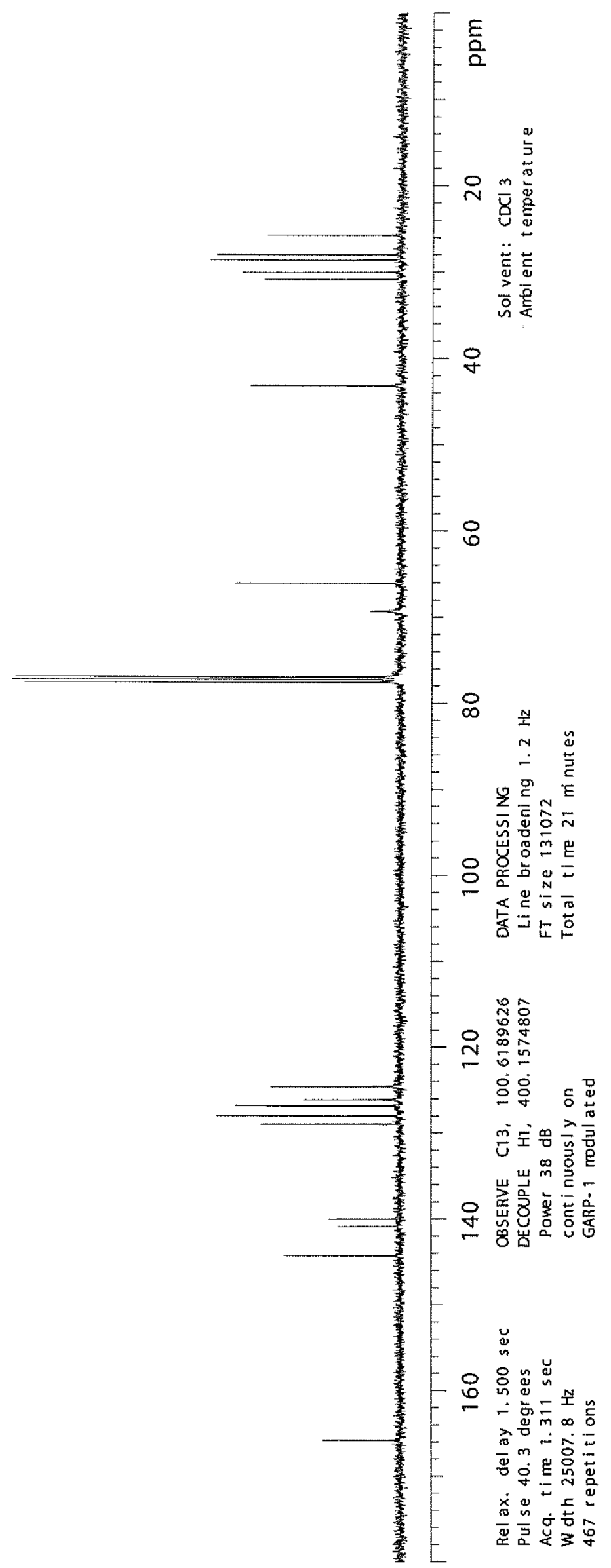




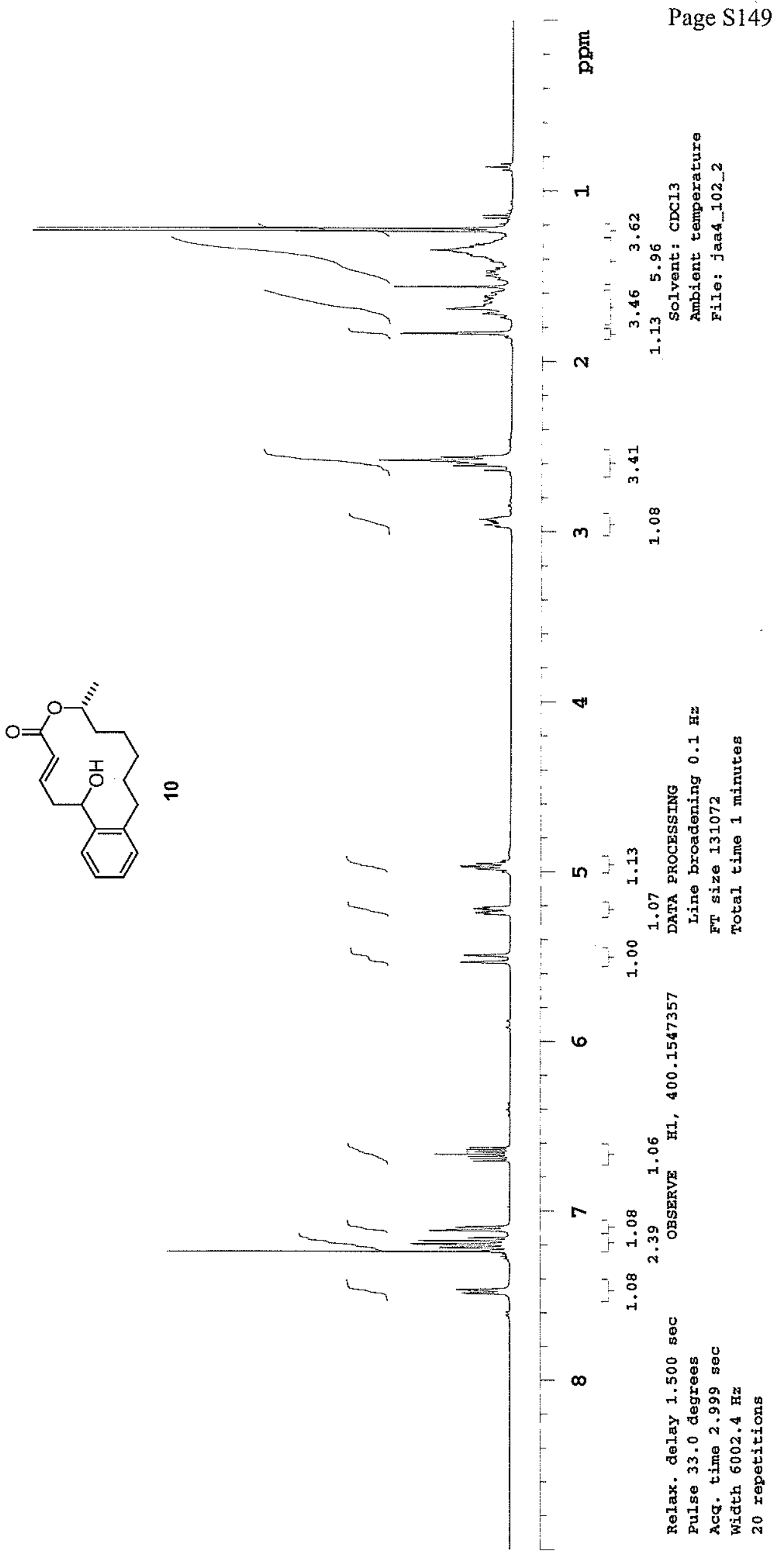


Page S150
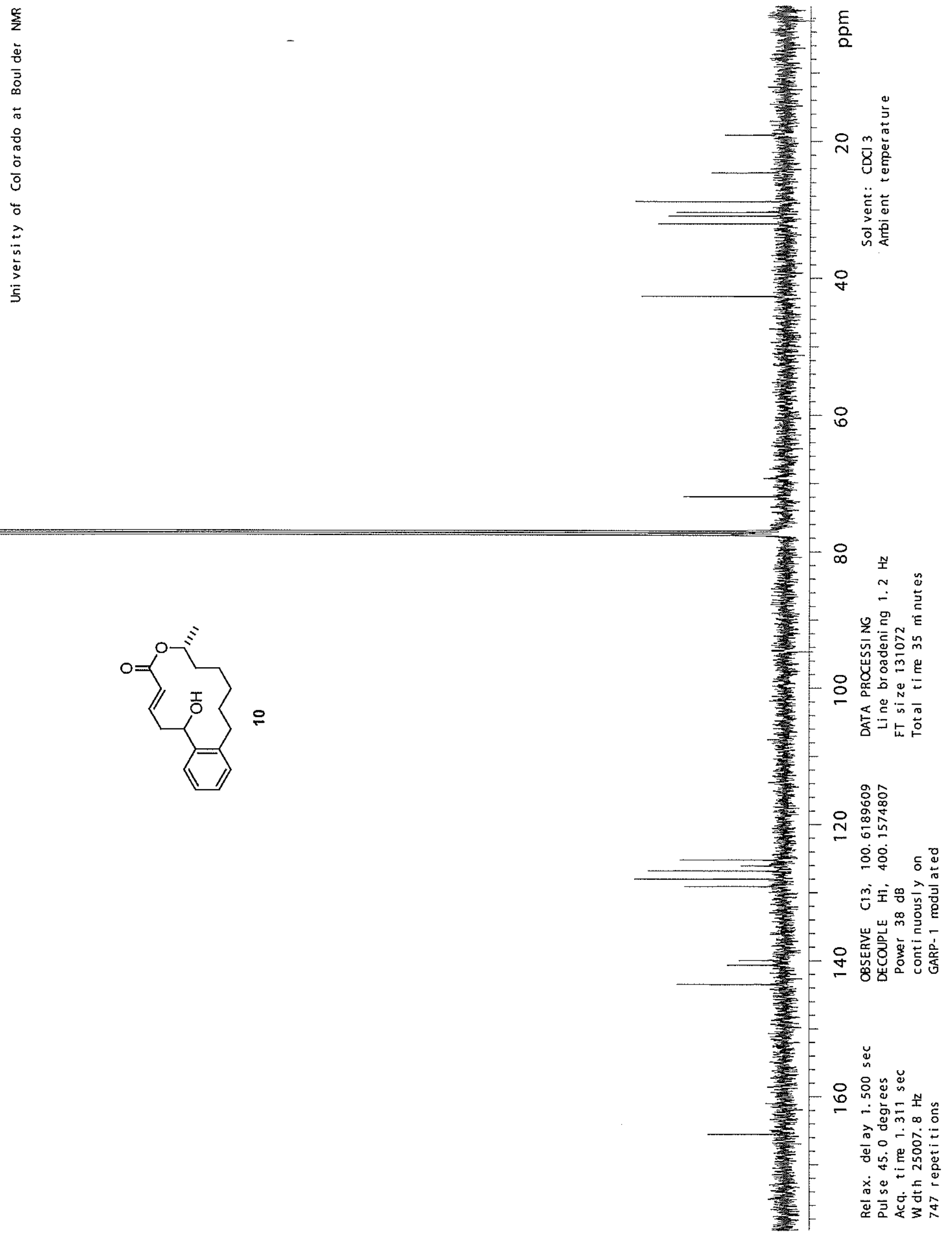


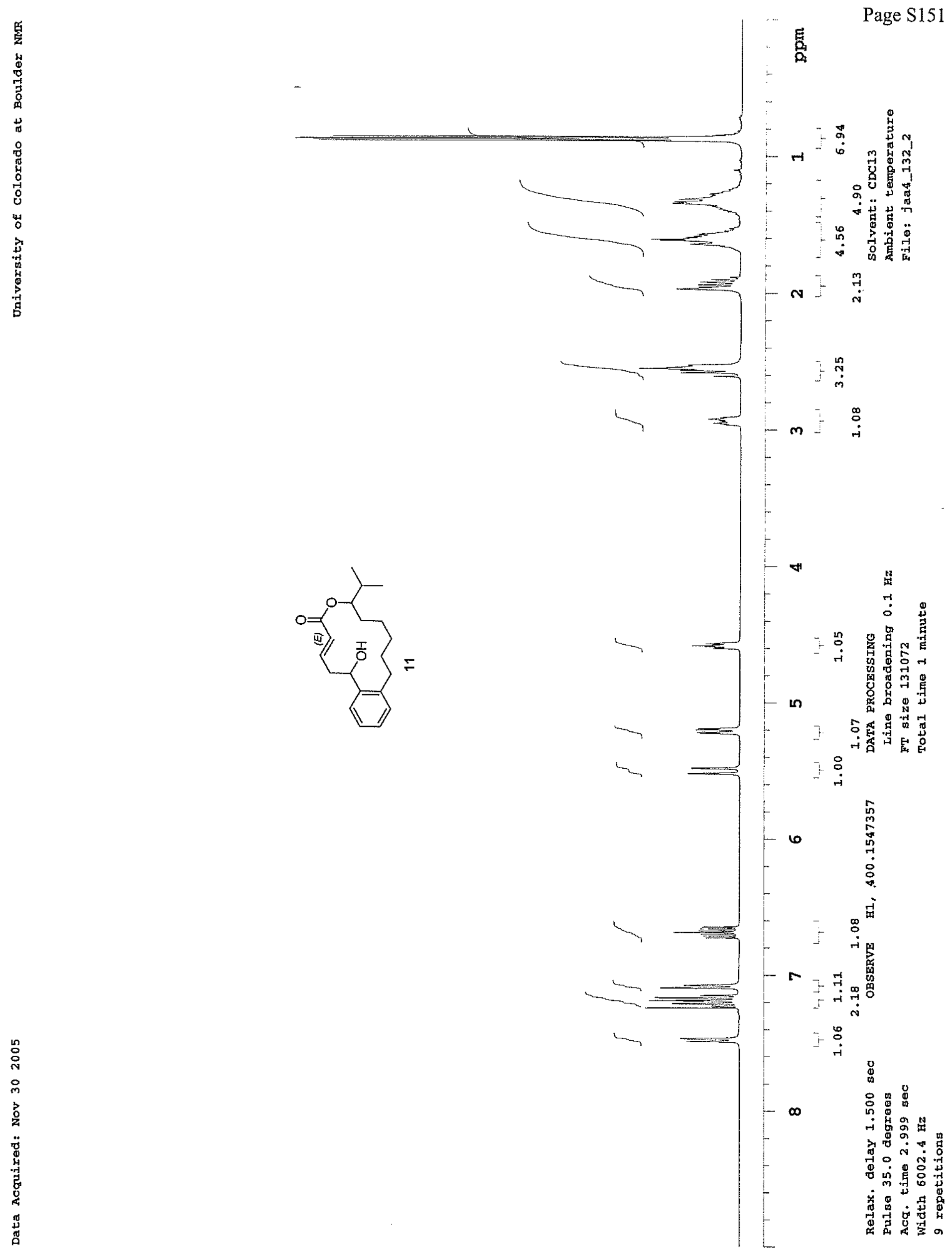


Page S152

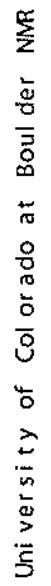

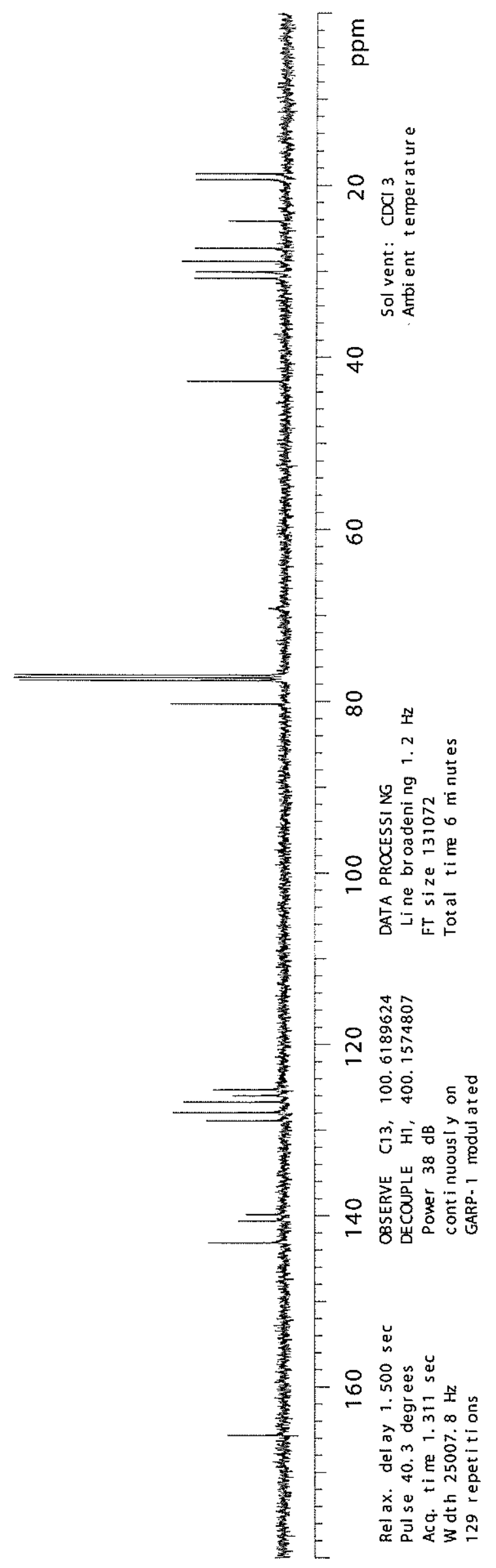



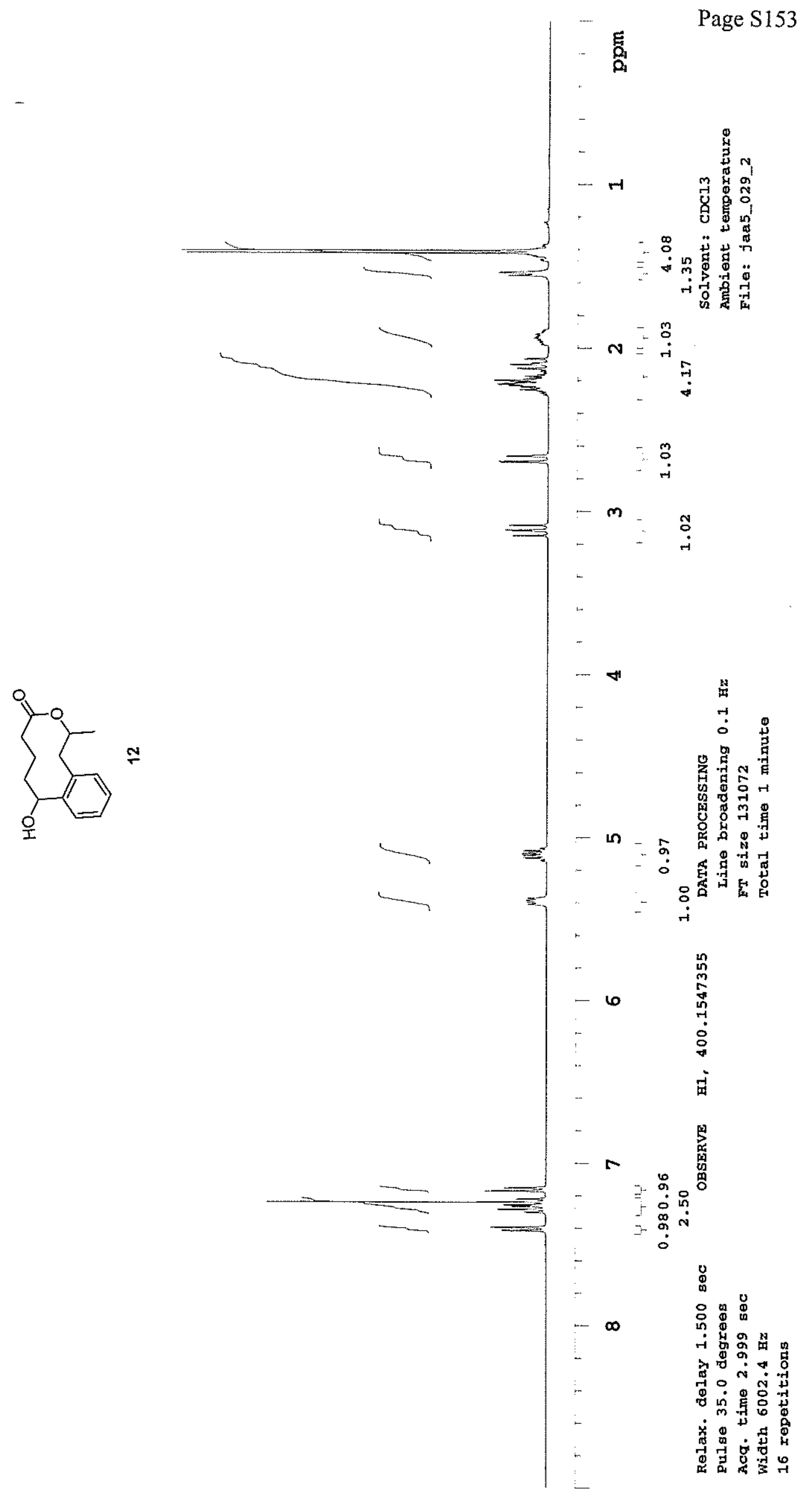
Page S154
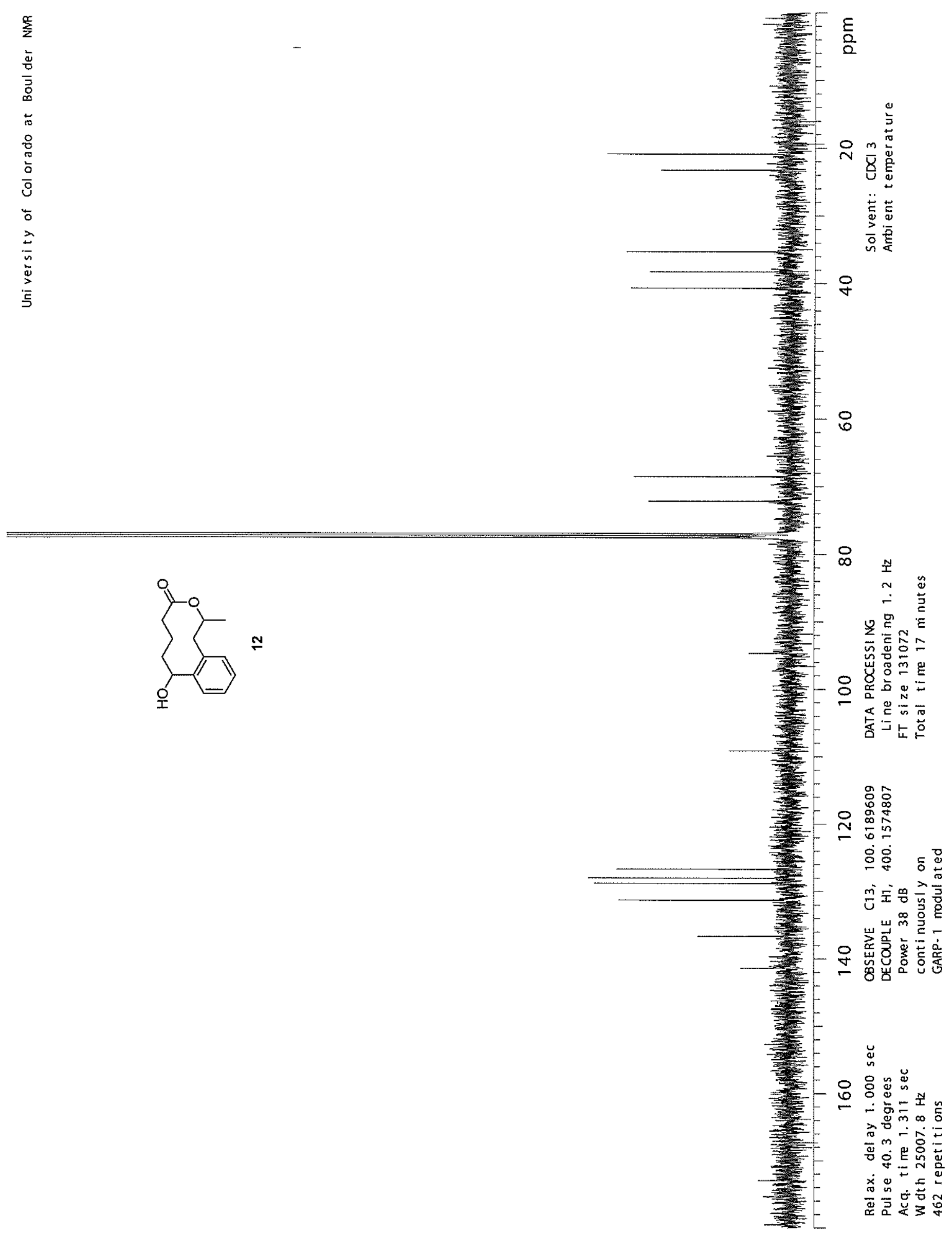


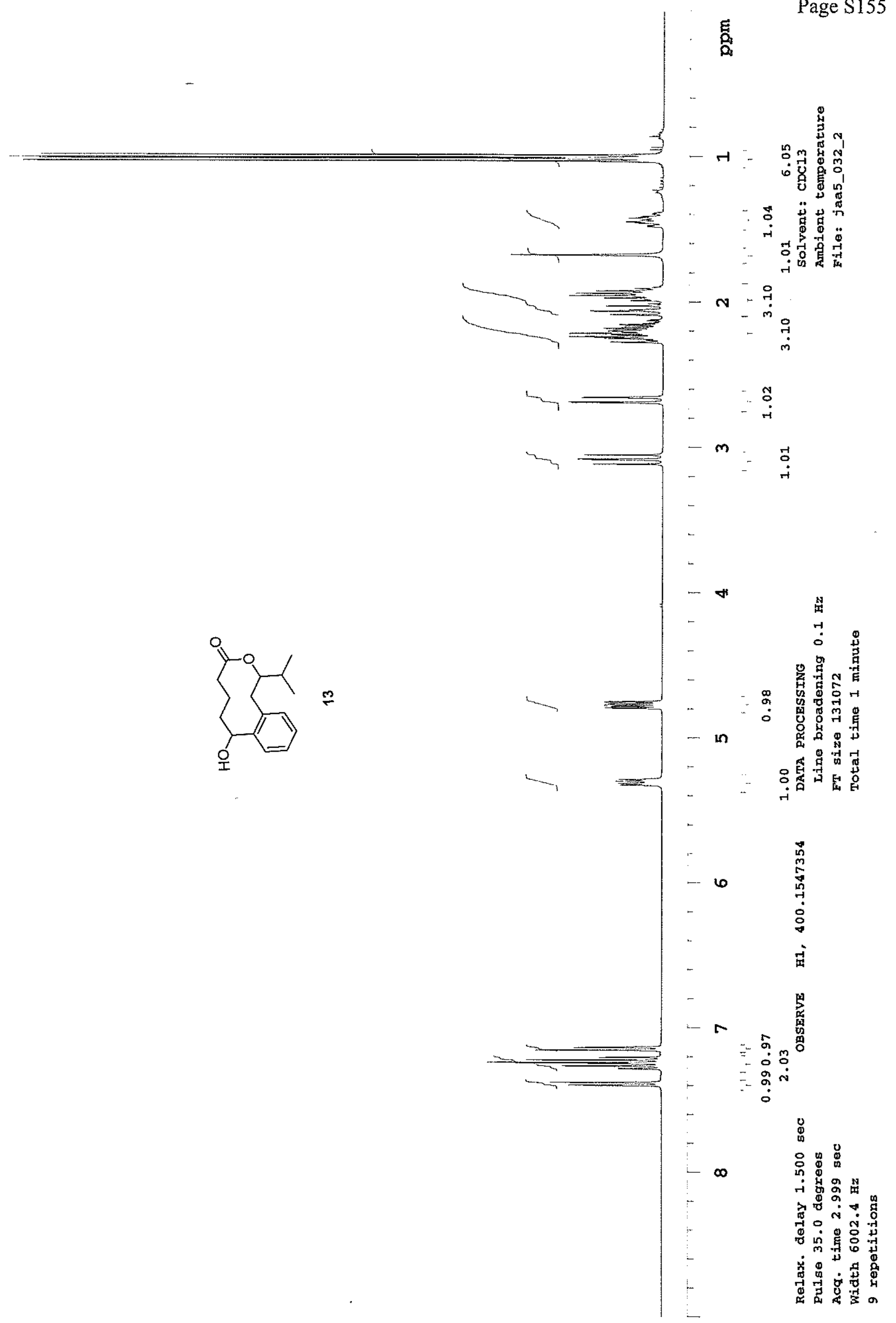




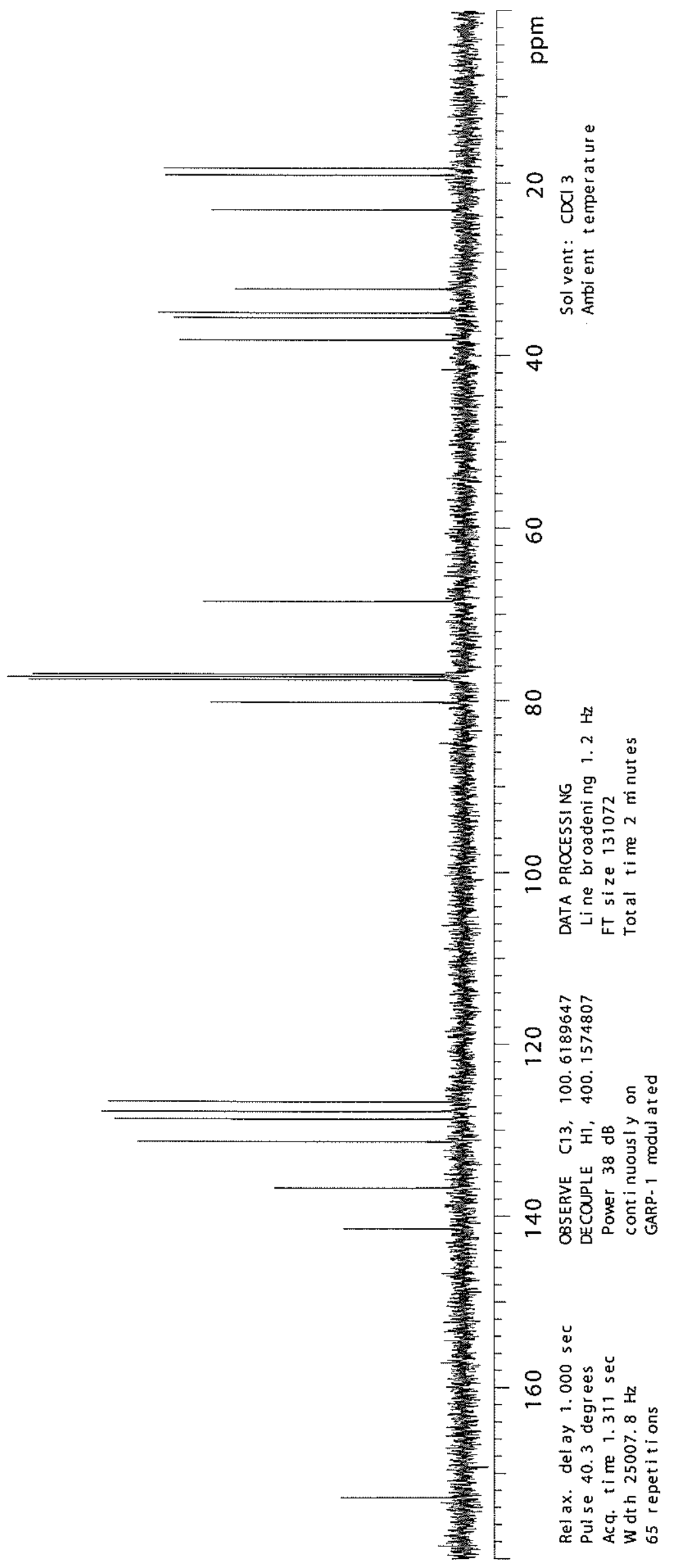




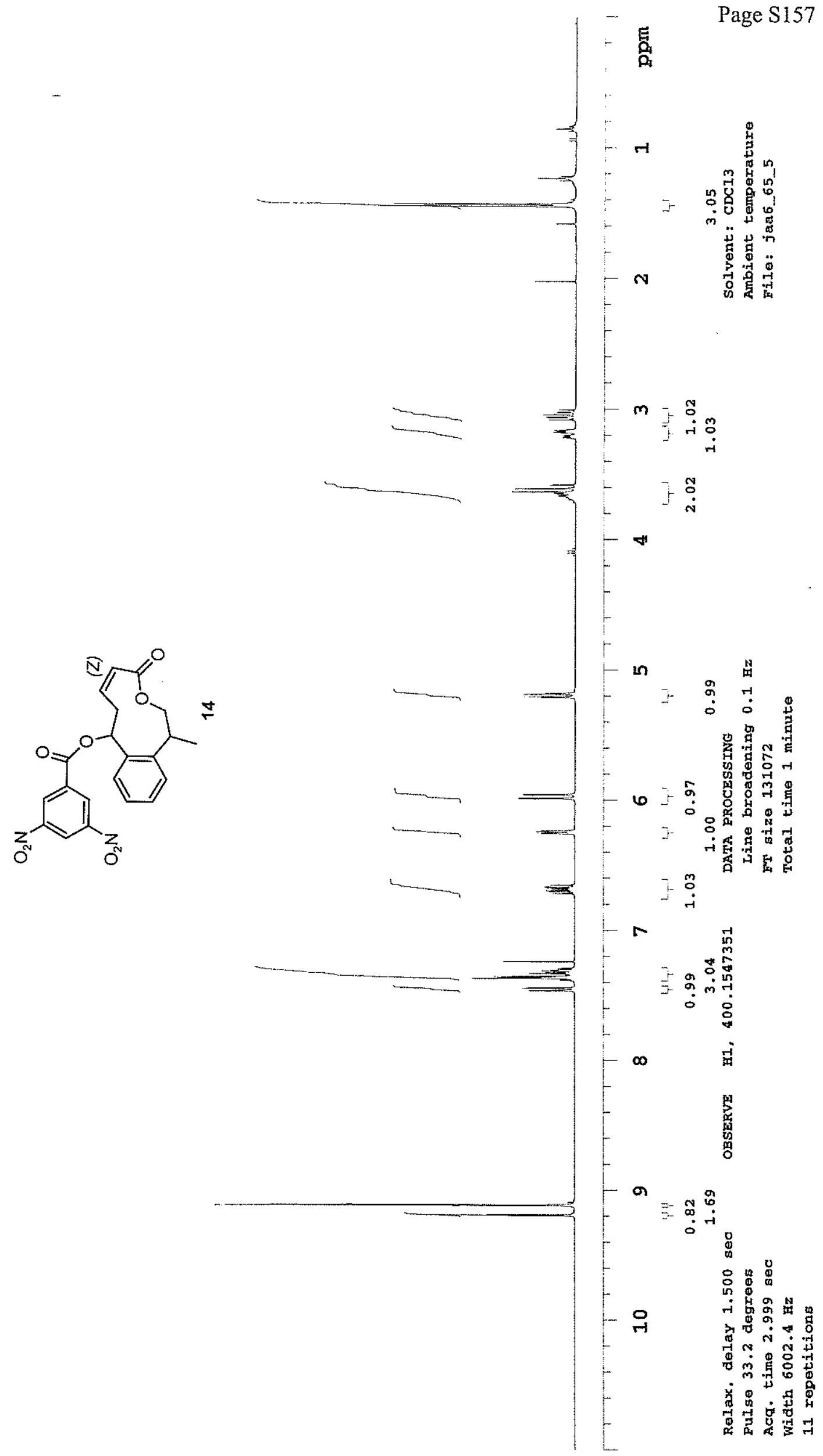




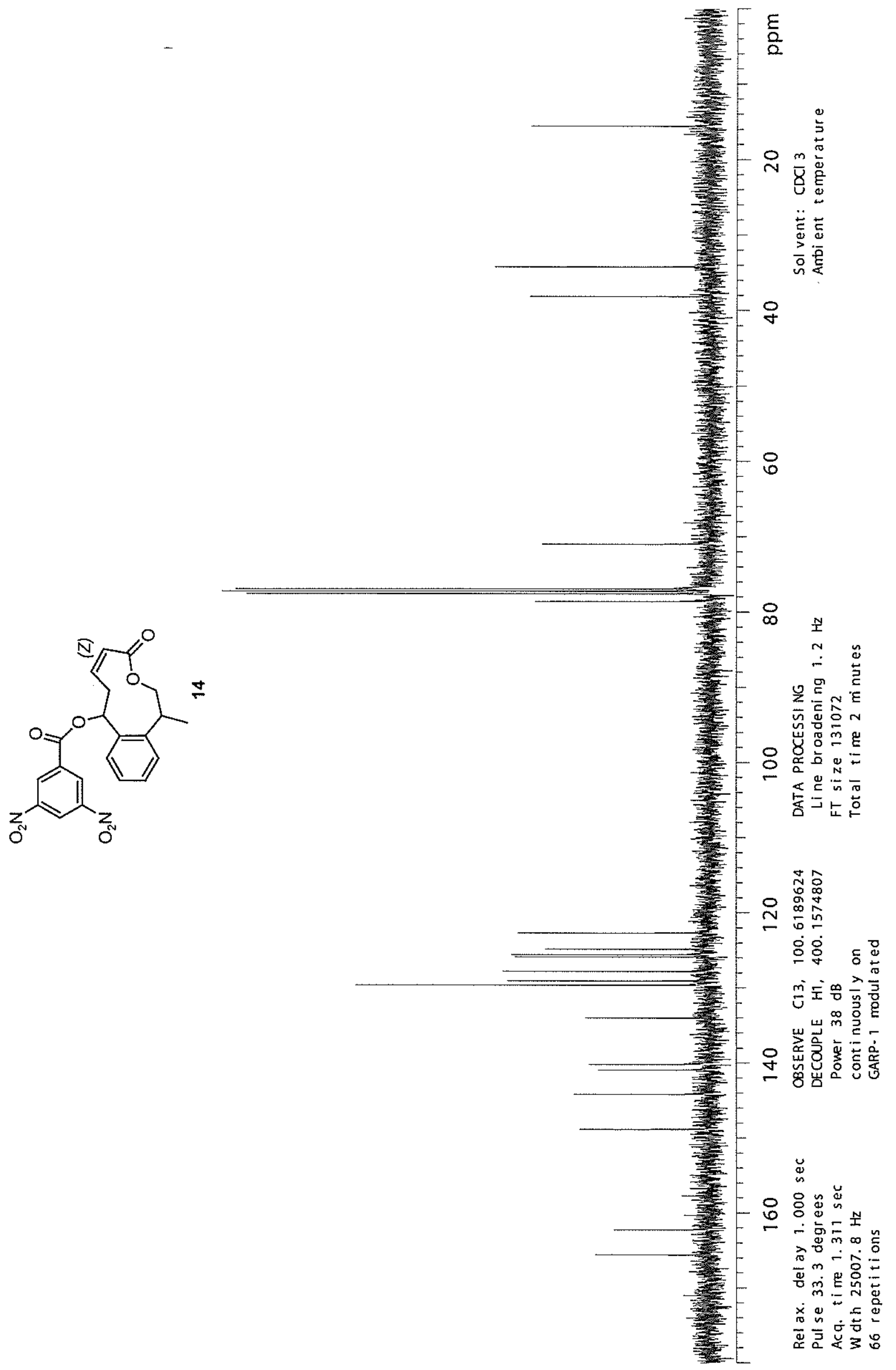




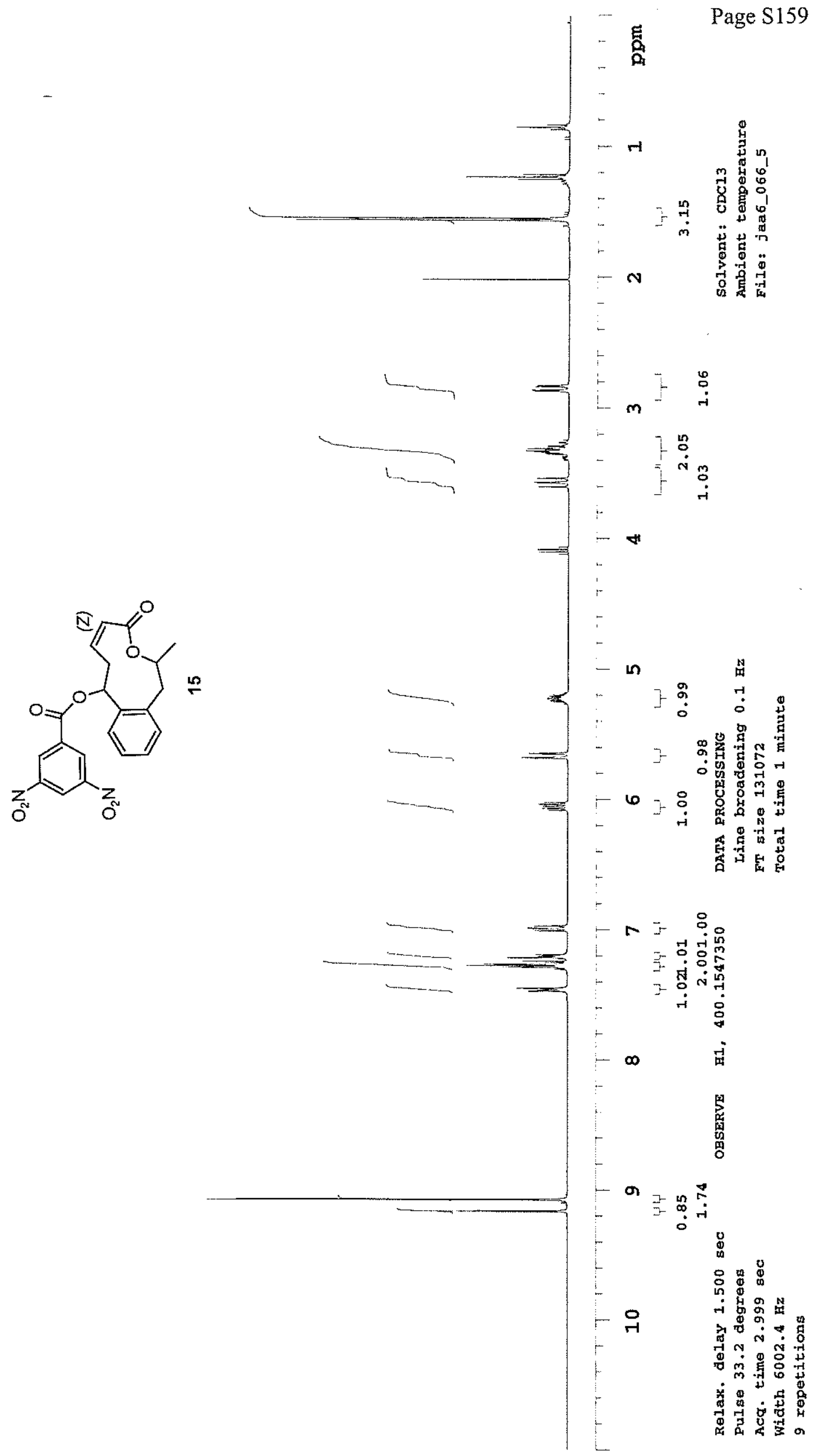


Page $\$ 160$

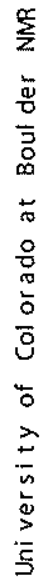

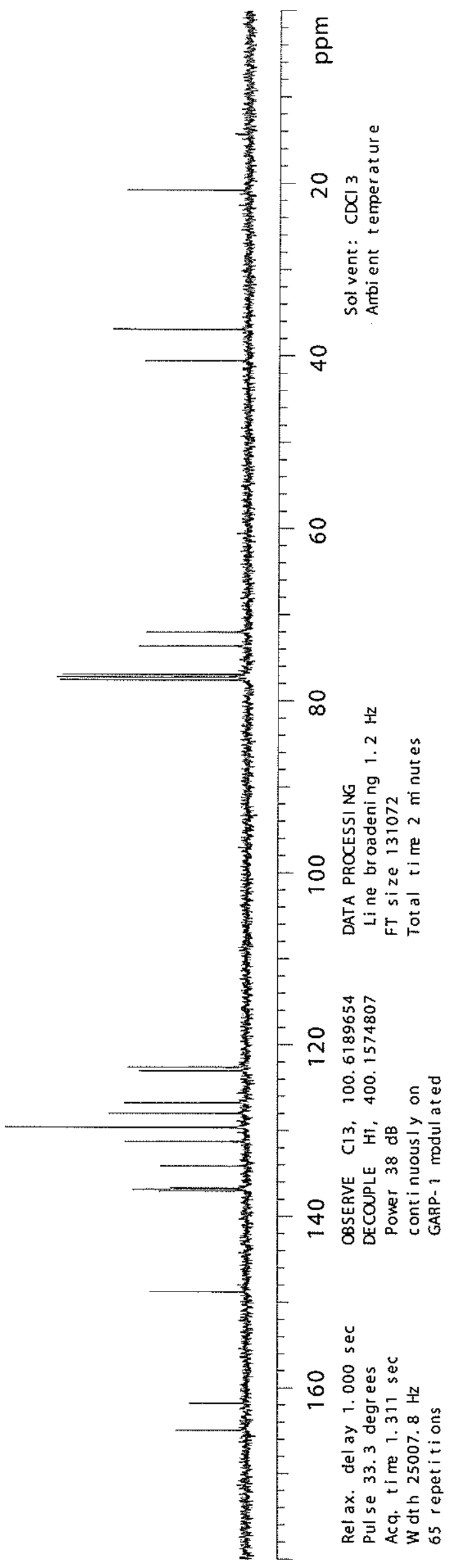




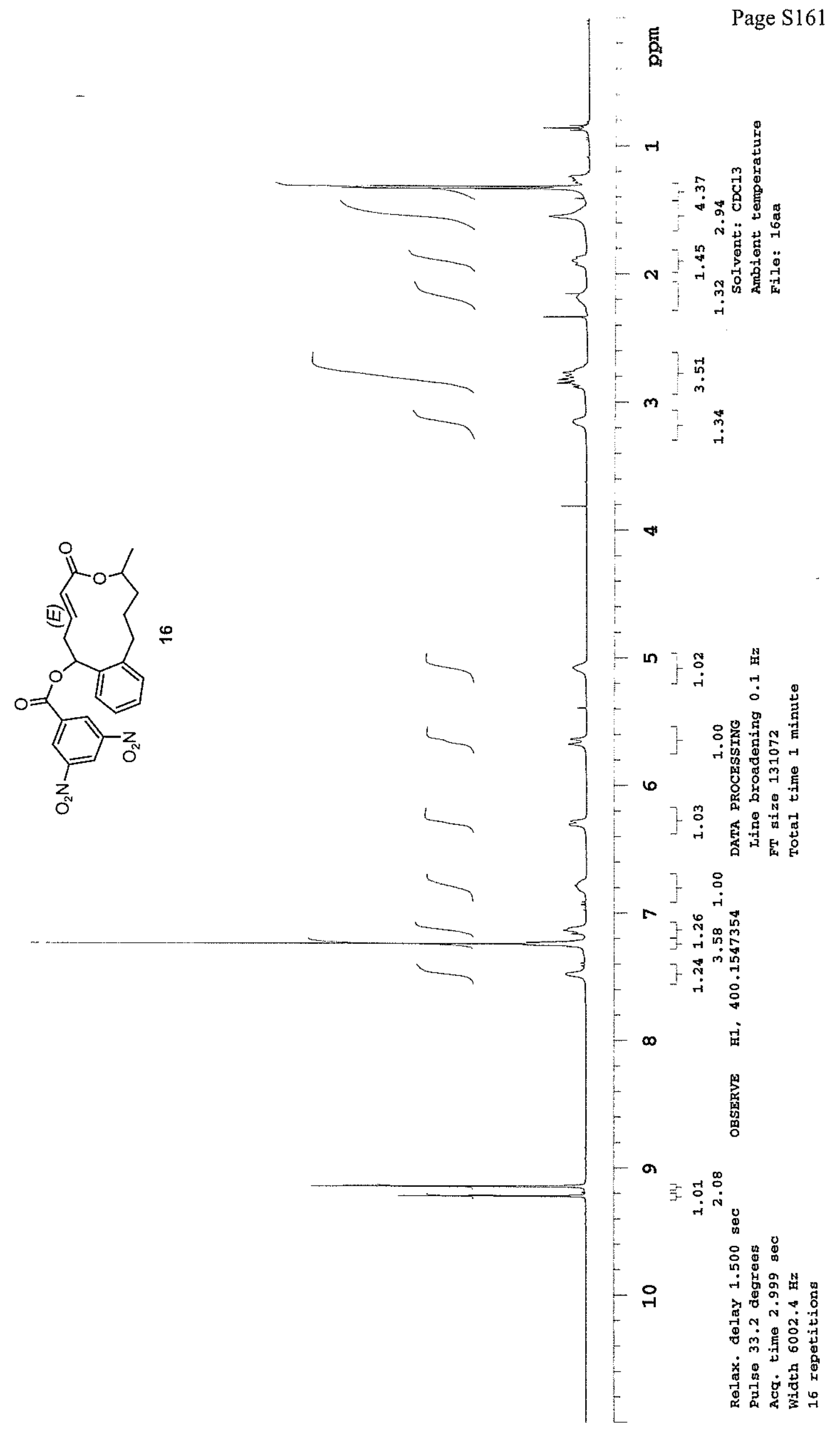




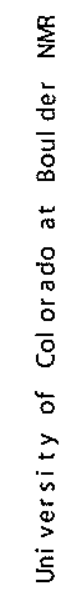

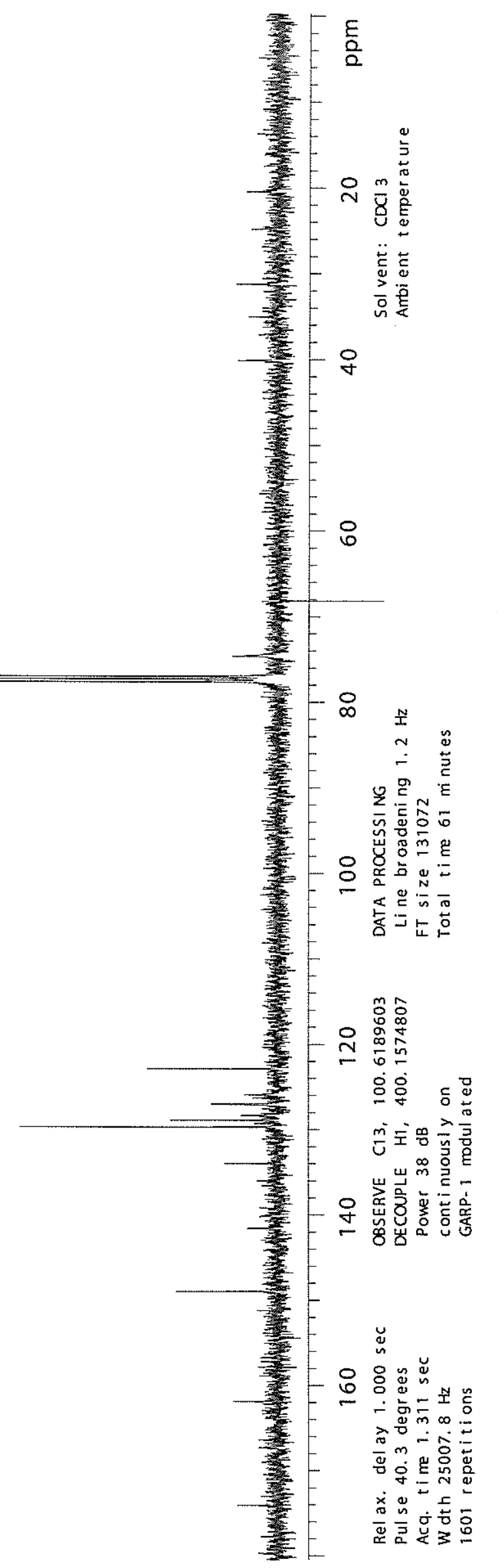




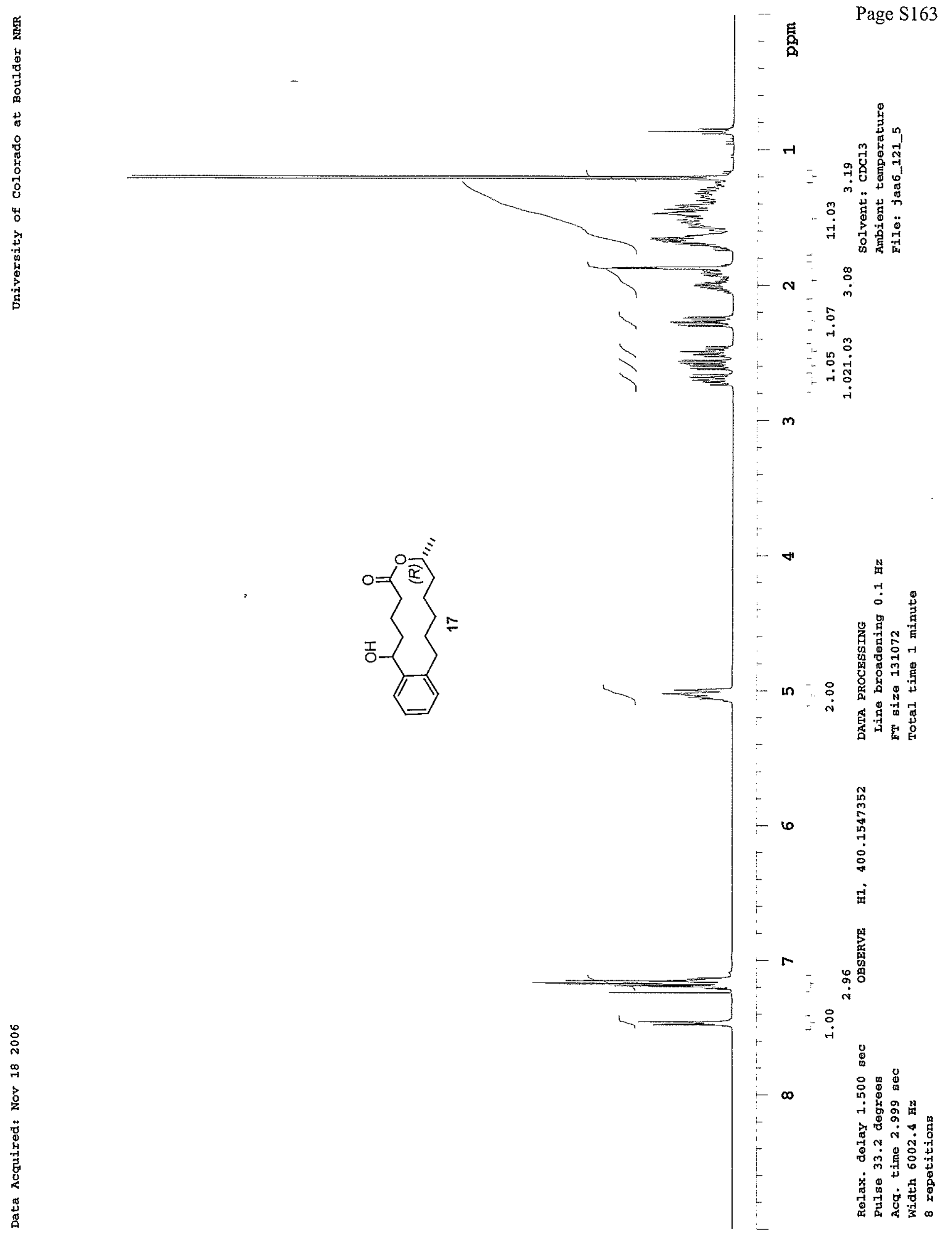




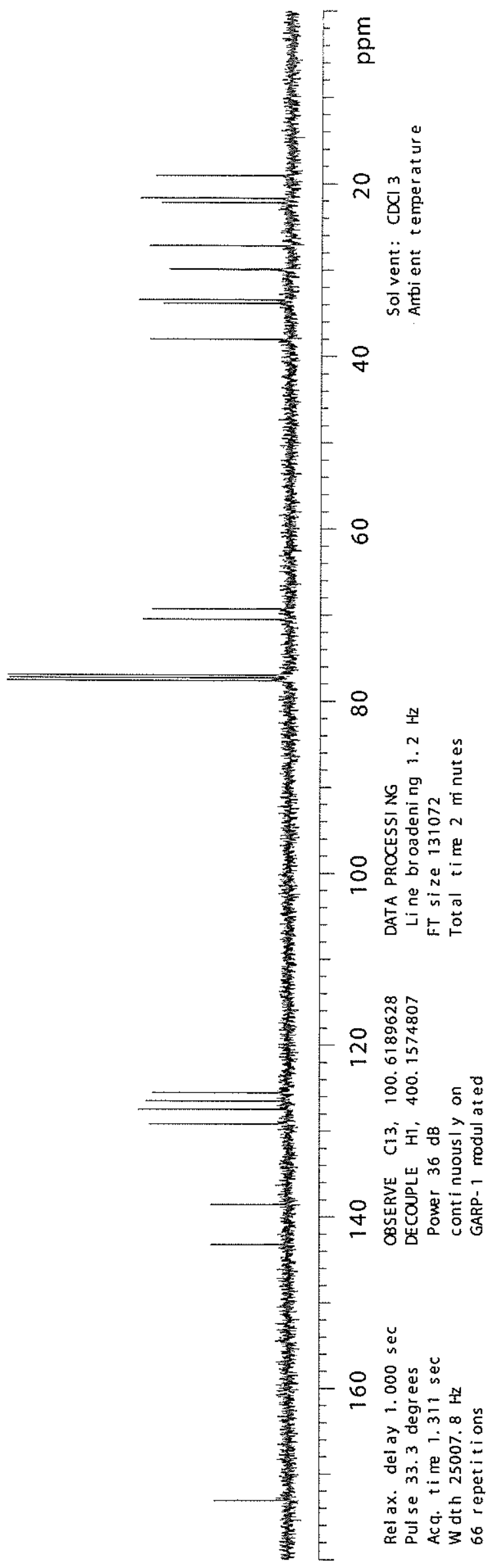




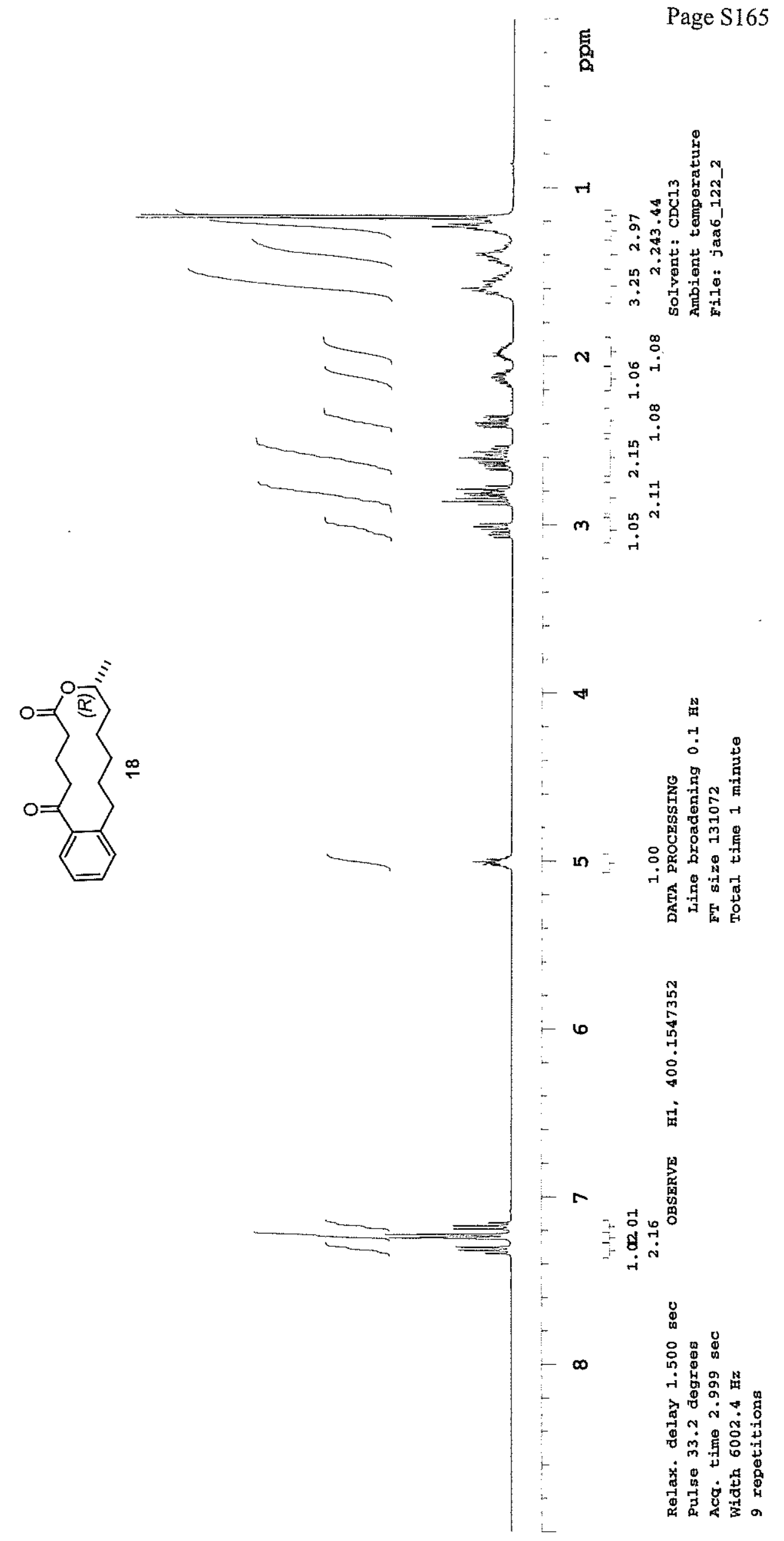


Page S166

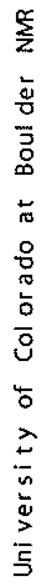

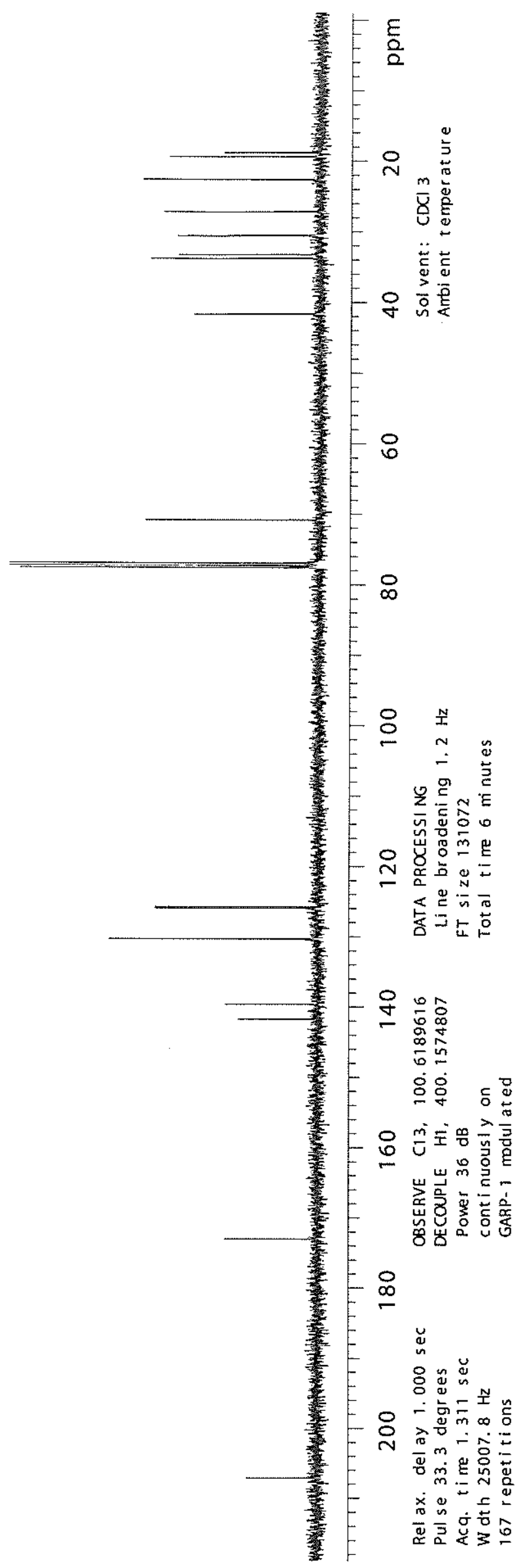




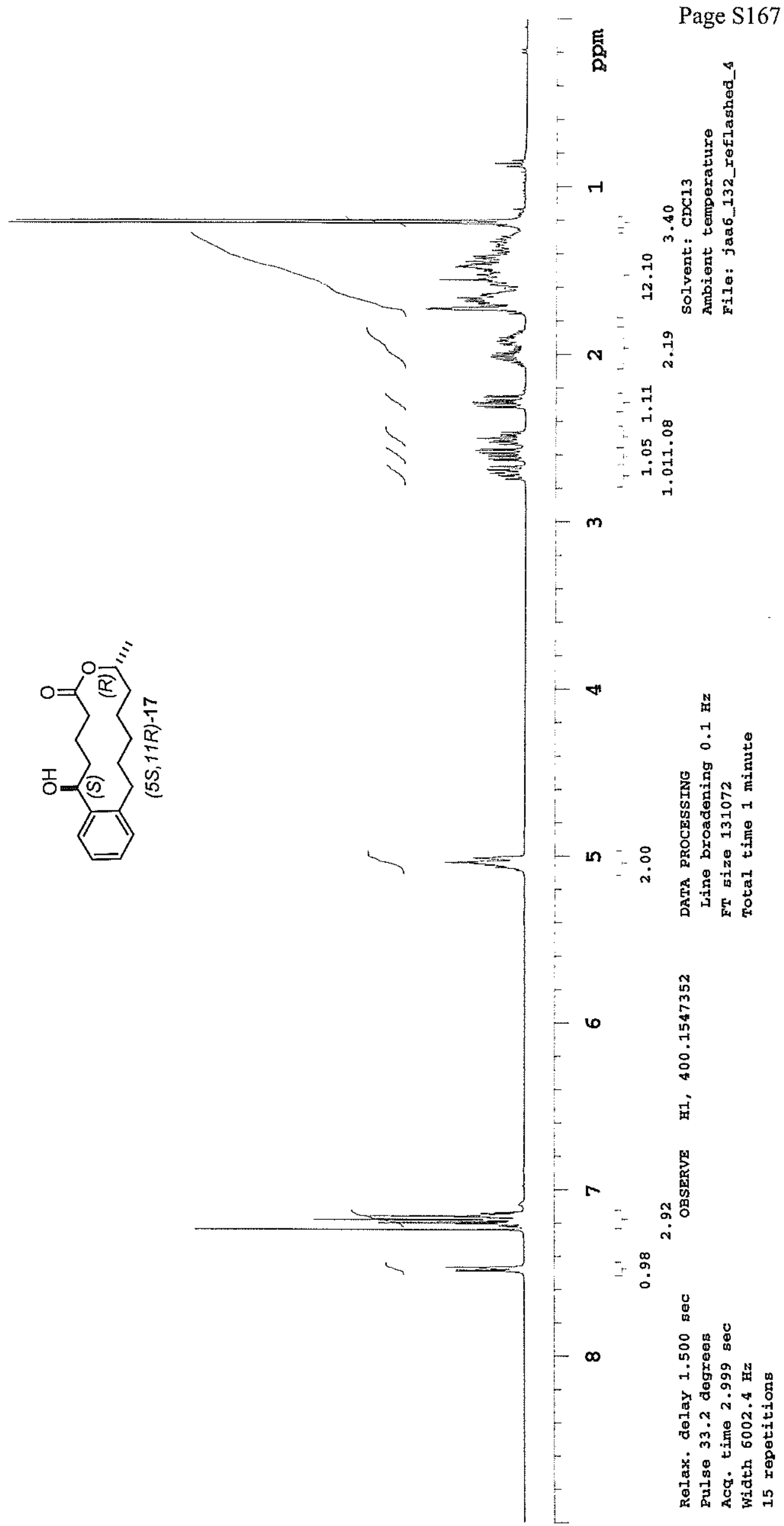



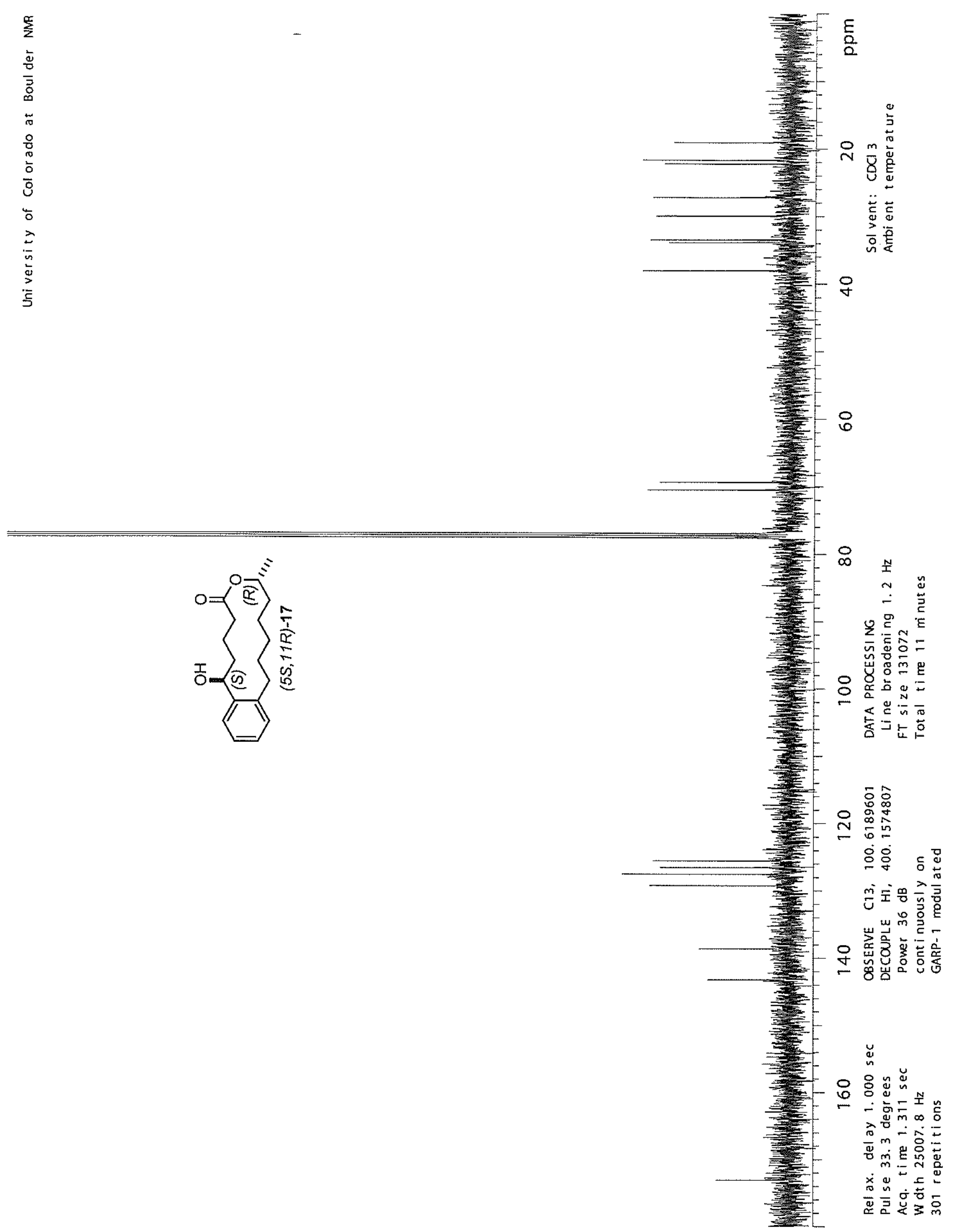


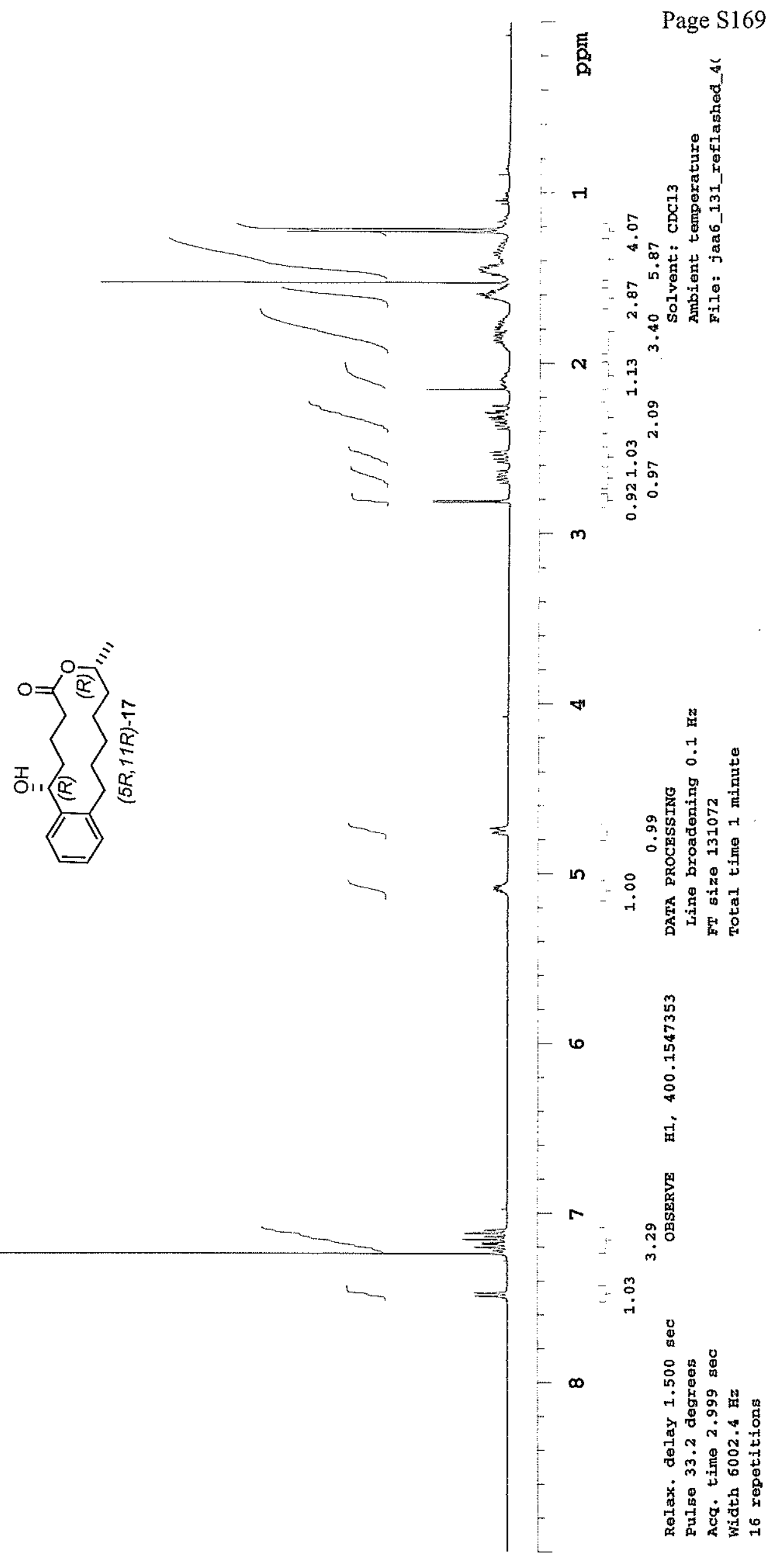


Page $\$ 170$
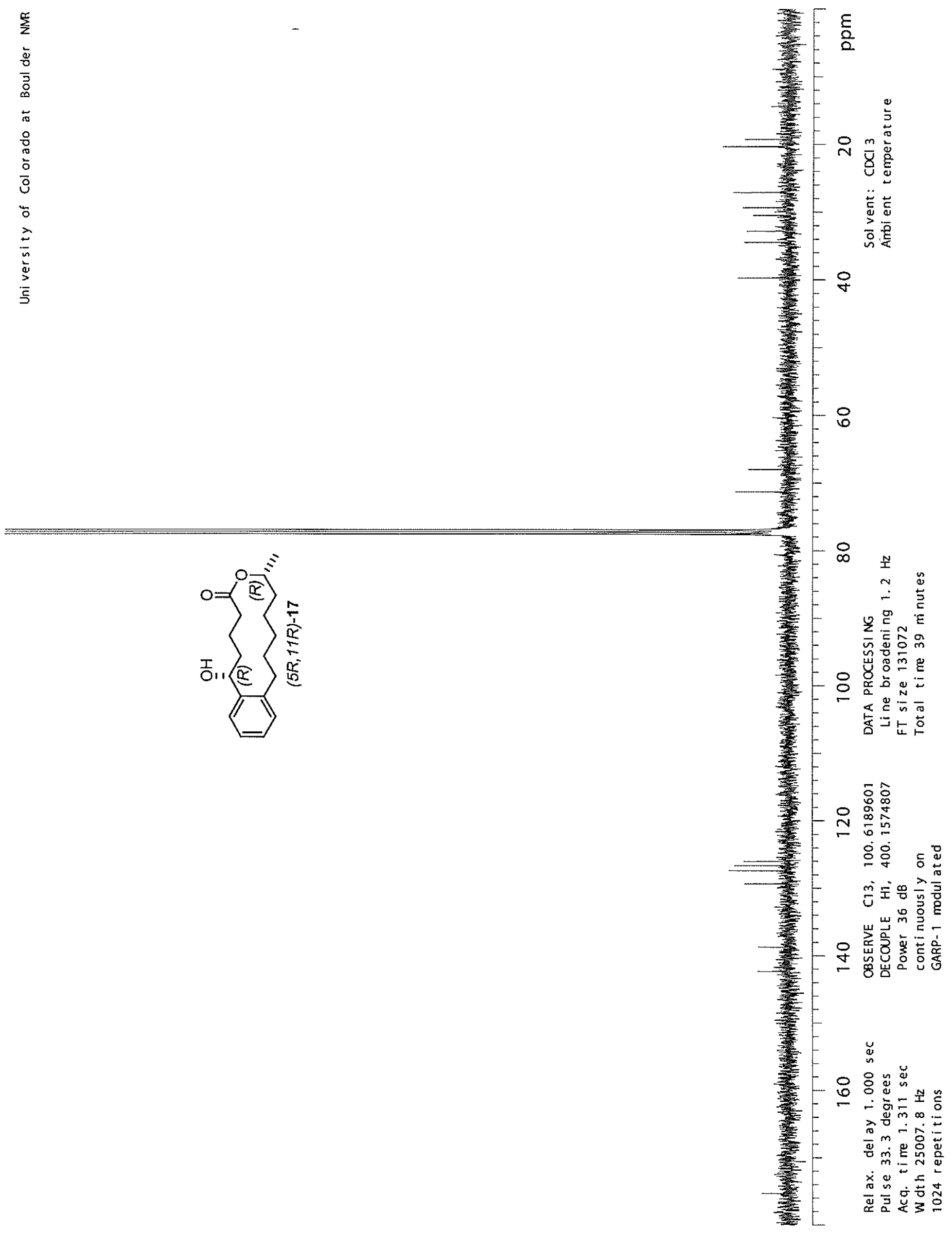